\begin{abstract}
SANTISO, ERIK EMILIO. Effect of Confinement on Chemical Reactions. (Under the direction of Keith E. Gubbins.)

The goal of this project is to gain a fundamental understanding of the different factors that influence chemical reactions in confinement through the systematic study of simple model systems. For this purpose, we consider three different examples: (1) The rotational isomerization of small hydrocarbons in carbon nanopores, showing the impact of the steric hindrance imposed by the confining material on the reacting system; (2) The unimolecular decomposition of formaldehyde on carbon pores, as an example of the effect of physical interactions on a reaction's mechanism and equilibrium; and (3) The thermal splitting of water over defective graphene and nanotube surfaces, showing how chemical interactions with a catalytic substrate can completely modify the chemical landscape and hence the equilibrium and dynamics of the system. Our main findings from these studies are: (1) In the molecular sieving limit, the steric hindrance imposed by the pore walls can cause dramatic changes in the potential energy surface of a reaction, with the rate varying as the double exponential of the pore dimensions; (2) Physical interactions with a catalytic support can alter both the thermodynamic properties of the confined molecules and the reaction dynamics, the latter being especially important in reactions with relatively low activation barriers; (3) Chemical modification of a carbon substrate can give rise to completely different reaction mechanisms, which could be exploited in the systematic molecular-level design of improved catalytic materials.
\end{abstract}




\title{
EFFECT OF CONFINEMENT ON CHEMICAL REACTIONS
}

by

\author{
ERIK EMILIO SANTISO \\ A dissertation submitted to the Graduate Faculty of \\ North Carolina State University \\ in partial fulfillment of the requirements for the \\ Degree of Doctor of Philosophy \\ CHEMICAL AND BIOMOLECULAR ENGINEERING \\ Raleigh, NC \\ 2007
}

APPROVED BY:

Keith E. Gubbins

Carol K. Hall

Chair of Advisory Committee

H. Henry Lamb

Marco Buongiorno Nardelli 


\section{Dedication}

A mi familia, y sobre todo a mis padres, por su apoyo incondicional en todo momento.

To my family, and especially my parents, for their unconditional support at all times. 


\section{Biography}

Erik E. Santiso was born on December 13, 1972 in Caracas, Venezuela, to his parents José Francisco Santiso and María Victoria Borrazás de Santiso, both originally from La Coruña, Spain. Erik grew up in Caracas, where he attended school at Colegio Santo Tomás de Villanueva from 1980 to 1989. In 1989 he got admitted into Universidad Simón Bolivar, and one year later he joined the Chemical Engineering program. While attending school and during his first three years in college, Erik also took music classes at the Juan Manuel Olivares School in Caracas and took private piano lessons, becoming proficient in several musical instruments and classical composition.

During his years in college, Erik developed a passion for teaching, which he has maintained ever since. He worked as an undergraduate teaching assistant in Mathematics from 1990 until 1995. He also joined Grupo Escalera, a non-profit student organization that offered low-cost classes to help high school students to get into college, and taught there in an honorary capacity from 1990 until 1995.

On September 1995, Erik graduated from college. Shortly thereafter, he started graduate studies in Chemical Engineering and joined the Faculty of the Department of Thermodynamics and Transport Phenomena at Universidad Simón Bolívar as a Lecturer. During this time, Erik was in charge of teaching a number of undergraduate courses, including Thermodynamics, Numerical Methods, and Transport Phenomena, and later also co-taught graduate courses. At the same time, Erik attended graduate classes and worked under the supervision of Professor Erich A. Müller in his research project. In this project, he used molecular simulation techniques to study the packing of dense mixtures of hard spheres, a problem related to the development of oil-in-water emulsions for use as alternative fuels. In 1999 Erik obtained his M.S. degree in Chemical Engineering (with honors) and became an Assistant Professor. 
In 2001, Erik traveled to Palo Alto, California, to visit the research group of Professor Abbas Firoozabadi, and had the opportunity to learn much about interfacial thermodynamics and the modeling of complex reservoir fluids. After a trip to Spain in the summer of 2002, Erik came to North Carolina and started his Ph.D. studies in Chemical Engineering at North Carolina State University. He then joined the research group of Professor Keith E. Gubbins, where he carried out the research presented in this book. During his years in NCSU, Erik also had the opportunity to continue teaching, working as a teaching assistant in undergraduate thermodynamics (Fall 2002 - Spring 2003), and later graduate mathematical modeling (Fall 2003, 2004, 2005 and 2006) and multiscale molecular modeling (Spring 2006). He also worked as a guest instructor in graduate thermodynamics (Fall 2006). After defending his dissertation, Erik will join the research group of Prof. Bernhardt Trout at the Massachusetts Institute of Technology, to work as a postdoctoral research associate. 


\section{Acknowledgements}

I want to first thank my advisor, Keith E. Gubbins, for offering me his guidance and support at all times. He has always given me the freedom to pursue my own ideas, while giving valuable suggestions at times when I did not see a clear way to go forward. I am also very grateful for his career advice - even though at times I would not be happy to hear it, he has always turned out to be right. Keith has also been eager to share with me his ideas and opinions about science, the academic world, and life in general, and I have often learned valuable lessons from him. Even though he has always encouraged me to aim high and do my best, he has also many times made me see things from a more realistic and practical point of view - something extremely valuable for an incorrigible idealist like myself. I am very thankful to Keith for giving me the opportunity to work in his group, for his constant support and advice, and for being not only an advisor, but also a good friend.

I would also like to thank all of the people I have had the privilege to work with, especially my main collaborators, Marco Buongiorno Nardelli, Aaron George, Liping Huang, Arthi Jayaraman, Milen Kostov, Sujata Paul and Heath Turner. I am grateful for all our discussions, their advice, and most importantly, their friendship. I am especially thankful to Marco for his constant encouragement and his contagious enthusiasm for our research, which has really helped me many times when I did not feel particularly thrilled about my work. I also want to express my gratitude to all of the exceptional scientists that I have had the opportunity to interact with: Supriyo Battacharya, Henry Bock, Naresh Chennamsetty, Benoit Coasne, Coray Colina, Francisco Hung, Surendra Jain, Joshua Moore, Tim Morrow, Jeremy Palmer, Jorge Pikunic, Lauriane Scanu, Flor Siperstein, Alberto Striolo, Victoria Wagoner, and all others that surely I am forgetting to mention as I write this. I am also grateful to my committee members, and to Jan Genzer, Greg Parsons, Tom Pearl and my former advisor and co-worker, Erich A. Müller for all of their support and advice. I especially want to thank Prof. Carol Hall for all of her support, especially in this 
particularly hard time of my life. I would also like to thank Prof. Peter Fedkiw for all the times we have worked together in his Mathematical Modeling class, and for giving me the opportunity to continue teaching. Also, I am especially thankful to Sandra Bailey and June McKoy for being so patient with my total lack of ability to keep up with paperwork, and for always being willing to help and answer all of my questions.

The work presented in this book was supported by grants from the National Science Foundation (DMF-0304299 and CTS-0211792), the U.S. Department of Energy (DEFG02-98ER14847 and DE-AC05-00OR22725), NATO (Collaborative Linkage Grant DEAC05-00OR22725), and the Petroleum Research Fund of the American Chemical Society. Supercomputing time was provided by the San Diego and Pittsburgh Supercomputing Center and the National Center for Supercomputing Applications under grants NSF/NRAC NPA205 and NSF/NRAC MCA93SO11, the High Performance Computing Centers at NCSU and UNC, and the Center for Computational Sciences at Oak Ridge National Laboratories.

Finally, I would also like to thank all of the friends who have made my stay in Raleigh so much more enjoyable, especially the current and former members of the Gubbins' and Hall's groups, as well as the Bock, Castaño-Colina, Hung, Nardelli and Scanu families. I am also very grateful to my family, and especially my parents, who have constantly given me their unconditional support and encouragement. This work would have not been possible without their help. 


\section{Table of Contents}

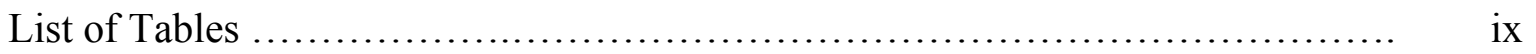

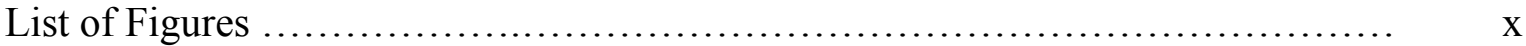

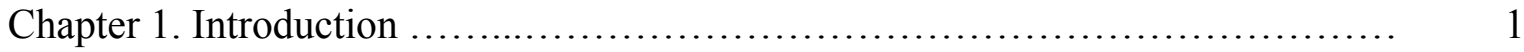

References and Notes ................................................. 4

Chapter 2. Review of Methods for Modeling Chemical Reactions ..................... 7

2.1. Introduction ...................................................... 7

2.2. Chemical Reactions ............................................... 9

2.3. Force Field Methods ............................................... 18

2.3.1. Semiempirical Force Fields ..................................... 19

2.4. Ab Initio Methods ................................................. 27

2.4.1. The Born-Oppenheimer Approximation .......................... 28

2.4.2. The Variational Principle ................................... $\quad 30$

2.4.3. Orbitals and Basis Functions ................................. $\quad 32$

2.4.4. Self-Consistent Mean-Field Theory - the Hartree-Fock Method ..... 39

2.4.5. Configuration Interaction and Other Variational Methods ........... 48

2.4.6. Perturbation Methods ............................................ 53

2.4.7. Coupled-Cluster Methods ..................................... 59

2.4.8. Semi-empirical Methods ........................................ 63

2.4.9. Density Functional Theory ..................................... 67

2.4.10. Ab Initio Molecular Dynamics ................................ 74

2.4.11. Quantum Mechanics/Molecular Mechanics ...................... $\quad 79$

2.5. Classical Simulation Methods for Equilibrium and Rate Constants .......... 82

2.5.1. Estimation of Equilibrium Constants ........................... 83

2.5.2. Transition State Theory and the Reactive Flux Method ............. 87

2.5.3. Constrained Reaction Dynamics: "Blue Moon" Method .............. 93

2.5.4. Transition Path Sampling ..................................... 102

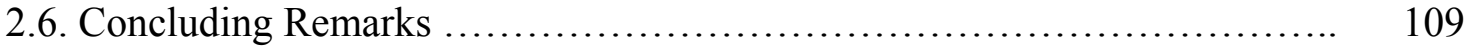

References and Notes ................................................... 111

Chapter 3. Isomerization of Small Hydrocarbons in Slit Pores and the Double

Exponential Effect ............................................. 152

3.1. Introduction ..................................................... 152

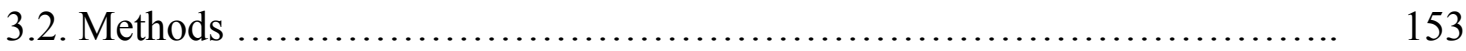

3.3. Results and Discussion ......................................... 155

3.3.1. n-Butane ................................................. 157

3.3.2. 1-Butene ................................................. 166

3.3.3. 1,3-Butadiene ................................................ 173

3.4. Concluding Remarks ............................................... 180

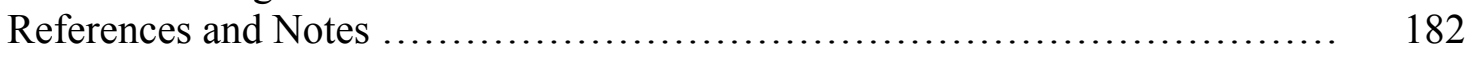


Chapter 4. Unimolecular Decomposition of Formaldehyde in Porous Carbons ...... 185

4.1. Introduction ..................................................... 185

4.2. Methods ........................................................... 186

4.3. Results and Discussion ............................................ 188

4.4. Concluding Remarks ............................................... 195

References and Notes .................................................. 196

Chapter 5. Dissociation of Water on Defective Carbon Surfaces ................... 199

5.1. Introduction ....................................................... 199

5.2. Thermal Splitting of Water on Defective Carbon Surfaces ................ 200

References and Notes .................................................... 211

Chapter 6. Conclusions and Future Research Directions ......................... 214

References and Notes .............................................. 217 


\section{List of Tables}

Table 3.1. Stable conformers of n-Butane, 1-Butene, and 1,3-Butadiene in the Bulk and within carbon slit pores. The C-C-C-C dihedral angle for each conformer is given in parenthesis below its name.

Table 3.2. C-C-C-C dihedral angles $(\omega)$ and energies of the stationary points for the rotational isomerization of $n$-butane in the gas phase and in carbon slit pores. Energies are in $\mathrm{kcal} / \mathrm{mol}$, and angles in degrees................

Table 3.3. Characteristic time (inverse of the rate constant) for the antigauche/anti-syn transition of butane at $300 \mathrm{~K}$ in the bulk and within graphene slit pores.

Table 3.4. C-C-C-C dihedral angles $(\omega)$ and energies of the stationary points for the rotational isomerization of 1-butene in the gas phase and in carbon slit pores. Energies are in $\mathrm{kcal} / \mathrm{mol}$, and angles in degrees.

Table 3.5. C-C-C-C dihedral angles (w) and energies of the stationary points for the rotational isomerization of 1,3-butadiene in the gas phase and in carbon slit pores. Energies are in $\mathrm{kcal} / \mathrm{mol}$, and angles in degrees.........

Table 4.1. Optimized geometries and energies of the stationary points along the reaction path for the dissociation of formaldehyde in vacuum. Energies are in $\mathrm{kcal} / \mathrm{mol}$, distances in $\AA$ and angles in degrees

Table 4.2. Optimized geometries and energies of the stationary points along the reaction path for the dissociation of formaldehyde above a graphene sheet (a) and within a slit pore of width $11 \mathrm{au}$ (b), as obtained from NEB calculations. The differences with the values for the bulk reaction are given in parentheses for comparison. Energies are in $\mathrm{kcal} / \mathrm{mol}$, distances in $\AA$ and angles in degrees

Table 4.3. Activation barriers and energies of reaction for the dissociation of formaldehyde within a carbon pore as a function of pore size, as obtained from NEB calculations. The values for the dissociation above the graphene sheet correspond to an infinite pore width. The differences with respect to the bulk reaction are given in parentheses for comparison. All the energies are in $\mathrm{kcal} / \mathrm{mol}$

Table 4.4. Comparison between the equilibrium distributions of reactants and products in the bulk ideal gas, above a graphene sheet, and within a 11 au graphite slit pore for different temperature intervals...................
Activation energies for the forward $\left(E_{A, f}\right)$ and backward $\left(E_{A, r}\right)$ reactions

Table 5.1. Activation energies for the forward $\left(E_{A, f}\right)$ and backward $\left(E_{A, r}\right)$ reaction
and total energy change $(\Delta E)$ for each of the reaction steps in planar graphene. 


\section{List of Figures}

Figure 2.2.1. A simple potential energy surface; contours are lines of constant energy. The variables $f(r)$ and $g(r)$ represent functions of the configuration. A and B are the reactant and product states, and TS is the transition state.

Figure 2.2.2. Contour plot of the potential energy surface for the reaction $\mathrm{O}+\mathrm{H}_{2}$

$\rightarrow \mathrm{H}+\mathrm{OH}$ as obtained with the data in ref. 27. The green line is the minimum energy path..........................................

Figure 2.2.3. Potential energy surface for $\mathrm{O}+\mathrm{H}_{2} \rightarrow \mathrm{H}+\mathrm{OH}$. The thick line is the minimum energy path.

Figure 2.2.4. The reaction path using the distance $\mathrm{H}-\mathrm{H}$ as a reaction coordinate. The dashed horizontal lines correspond to a constant reaction coordinate. The triangles are the minimum energy points obtained in the forward direction, and the circles are the points obtained in the backwards direction.

Figure 2.2.5. The reaction path using the difference between the $\mathrm{H}-\mathrm{H}$ and $\mathrm{O}-\mathrm{H}$ distances as a reaction coordinate. The dashed diagonal lines correspond to a constant reaction coordinate. The circles are the minimum energy points found on the lines of constant reaction coordinate.

Figure 2.3.1. Energy contributions in a typical force field..................... 20

Figure 2.4.1. Three successive approximations to a hydrogen 1s Slater orbital using Gaussian functions..................................... 36

Figure 2.4.2. The Hartree-Fock picture. The original many-body problem is transformed into a set of 2-body problems by replacing all but one of the electrons by a mean field. The equations for each electron are then solved self-consistently

Figure 2.4.3. Connection between many of the $a b$ initio methods. The terms in this schematic are explained in the following sections...

Figure 2.4.4. Excited configurations. HOMO and LUMO stand for "highest occupied molecular orbital" and "lowest unoccupied molecular orbital"

Figure 2.4.5. Geometries and energies of the reactant, products and transition state for the 1-step diazetization of $\mathrm{C}_{7} \mathrm{H}_{10} \mathrm{~N}_{2}$. The energies shown were obtained using the CASPT2 method, values in parentheses are from density functional theory calculations. From ref. $225 \ldots \ldots \ldots \ldots \ldots \ldots$

Figure 2.4.6. The Kohn-Sham approach.

Figure 2.4.7. Sketch of a QM/MM system, showing the kinds of interactions that contribute to each part of the Hamiltonian in equation (2.4.75)........ 
Figure 2.5.1. Sampling in the Blue Moon method. The thick line represents the phase space trajectory with the reaction coordinate constrained, which is used to obtain the second factor in (2.5.12). The thin lines are unconstrained trajectories starting at configurations from the constrained simulation; these are used to obtain the first factor in (2.5.12). From ref. 450

Figure 2.5.2. For simple reactions that do not involve many degrees of freedom it is usually easy to determine the reaction mechanism by locating the energy minima and saddle points (left). In complex reaction where many degrees of freedom are involved there may be saddle-points and local minima, and hence many possible reaction pathways (right). From ref. 468

Figure 3.1. Snapshots of the stable conformers of n-butane, 1-butene, and 1,3butadiene as obtained from our DFT calculations. The non-planar conformations shown correspond to the bulk molecules

Figure 3.2. Torsional potential of an isolated n-butane molecule as obtained from our DFT-BLYP calculations. The open triangles denote the four stationary points (syn, gauche, eclipsed, and anti), and labels indicate the corresponding values of energy and torsion angle. The black circles denote results of constrained geometry optimizations at various torsion angles. The line is a spline fit to the data...

Figure 3.3. Torsional potential of a n-butane molecule confined within carbon slit pores as obtained from our DFT-BLYP calculations. The open triangles denote the stationary points. The lines are spline fits to the data, and labels adjacent to the lines indicate the corresponding pore widths....

Figure 3.4. Arrhenius plots for the VTST rate constant of the anti-gauche/antisyn transition in n-butane in the ideal gas phase and within carbon slit pores. The labels to the right of each line indicate the pore size....

Figure 3.5. The double exponential effect: a plot of $\ln \left[\ln \left(k_{\infty} / k\right)\right]$ vs. pore width at $300 \mathrm{~K}$ for $\mathrm{n}$-butane using the data from figure 3.4. The black circles correspond to our calculation results, and the line is a linear fit to the data

Figure 3.6. Torsional potential of an isolated 1-butene molecule as obtained from our DFT calculations. The open triangles denote the four stationary points (syn, gauche, skew and anti), and labels indicate the corresponding values of energy and torsion angle. The black circles denote results of constrained geometry optimizations at various torsion angles. The line is a spline fit to the data... 
Figure 3.7. Torsional potential of a 1-butene molecule confined within carbon slit pores as obtained from DFT-BLYP calculations. The open triangles denote the stationary points. The lines are spline fits to the data, and labels adjacent to the lines indicate the corresponding pore widths..........................................................

Figure 3.8. Effect of confinement on the isomerization rate of 1-butene as obtained from our VTST calculations. The line labeled "Bulk" corresponds to the isolated molecule (ideal gas). The other lines correspond to isolated molecules confined in carbon slit pores. The labels next to the lines indicate the corresponding pore widths........

Figure 3.9. The double exponential effect: a plot of $\ln \left[\ln \left(k_{\infty} / k\right)\right]$ vs. pore width at $300 \mathrm{~K}$ for 1-butene using the data from figure 3.8. The black circles correspond to our calculation results, and the line is a linear fit to the data...................................................... 169

Figure 3.10. Torsional potential of an isolated 1,3-butadiene molecule as obtained from our DFT-BLYP calculations. The open triangles denote the four stationary points (s-cis, gauche, TS and s-trans), and labels indicate the corresponding values of energy and torsion angle. The black circles denote results of constrained geometry optimizations at various torsion angles. The line is a spline fit to the data.............

Figure 3.11. Torsional potential of a 1,3-butadiene molecule confined within carbon slit pores as obtained from our DFT-BLYP calculations. The open triangles denote the stationary points. The lines are spline fits to the data, and labels adjacent to the lines indicate the corresponding pore widths.................................................

Figure 3.12. Effect of confinement on the isomerization rate of 1,3-butadiene as obtained from our VTST calculations. The line labeled "Bulk" corresponds to the isolated molecule (ideal gas). The other lines correspond to isolated molecules confined in carbon slit pores. The labels next to the lines indicated the corresponding pore widths........

Figure 3.13. The double exponential effect: a plot of $\ln \left[\ln \left(k_{\infty} / k\right)\right]$ vs. pore width at $300 \mathrm{~K}$ for 1,3-butadiene using the data from figure 3.12. The black circles correspond to our calculation results, and the line is a linear

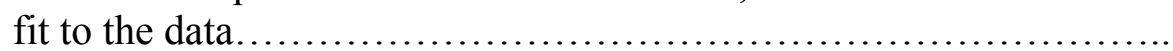

Figure 4.1. Energetics for the dissociation of formaldehyde over a graphene sheet (top) and within a 11 au (5.82 $\AA$ ) wide pore (bottom). A van't Hoff plot comparing the equilibrium distribution of reactants and products for the decomposition of formaldehyde happening in a bulk ideal gas, above a graphene sheet, and within a graphite slit

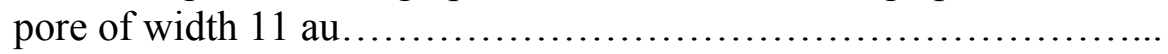


Figure 5.1. Ideal-gas equilibrium yields (fraction of the water that is converted to hydrogen at equilibrium in $\%$ mole) for the dissociation of water in the bulk (blue), over a single vacancy in graphene (green) and over a single vacancy in a 10-10 carbon nanotube (pink). The values correspond to a pressure of 1 bar and an initial equimolecular mixture of water and nanotubes

Figure 5.2. Top and side views of various intermediate states in the water splitting reaction over a vacancy site in graphene. (a) initial physisorbed state; (b) IM1: spin-singlet state with the highest energy; (c) IM2: spin-singlet state; (d) IM3: the only spin-triplet state $(\mathrm{S}=1)$; (e) IM4: spin-singlet state; (f) $K$ (for "ketone"): spin-singlet state; (g) $E$ (for "ether"): a spin-singlet. This is the global minimum of the potential energy surface; (h) $F$ (for "final"): spin-singlet state; the oxygen occupies the vacancy site on the surface and hydrogen is away from it.

Figure 5.3. Contour plot of the PES for the water dissociation reaction on a vacancy in graphene, as obtained by interpolation of the NEB trajectories. Different configurations are labeled as in Fig. 5.2. The order parameters $\phi_{1}$ and $\phi_{2}$ are defined as $\phi_{1}=(<\mathrm{CO}>-<\mathrm{OH}>) / \sqrt{ } 2$ and $\phi_{1}=(<\mathrm{CO}>+<\mathrm{OH}>) / \sqrt{ } 2$, where $<\mathrm{CO}>$ is the average distance between the oxygen atom and the carbons immediately adjacent to the vacancy, and $<\mathrm{OH}>$ is the average distance between the oxygen atom and the hydrogen atoms

Figure 5.4. Top and side views of (a) the initial $(N-I),(\mathrm{b})$ intermediate $(N-I M 2)$, (c) transition state $(N-T S T)$, and (d) final $(N-F)$ conformations for a favorable dissociation pathway over a vacancy in a $(10,10)$ nanotube... 


\section{Chapter 1}

\section{Introduction}

Most chemical reactions of practical interest are carried out in micro- and nanoporous materials, which can enhance or reduce reaction yields through various different effects, including an increase in the surface area per unit volume, selective adsorption of reactants and/or products, and shape-catalytic effects, among others. A fundamental understanding of the role of each one of these different effects could lead to a systematic procedure for the design of improved catalytic materials that take advantage of all of them simultaneously ${ }^{1}$.

Despite the multitude of factors that can affect reactions in confinement, we can classify these effects into three main groups: (1) shape-catalytic effects, i.e. the effect of the shape of the confining material and/or the reduced dimensionality of the porous space, (2) physical (or "soft") effects, including the influence of dispersion and electrostatic interactions with the confining material, and (3) chemical (or "hard”) effects, interactions that involve significant electron rearrangement, including the formation and breaking of chemical bonds with the confining material. The latter is usually considered to be the actual catalytic effect, and it is the one that has the most obvious influence on the reaction rates, as it alters the reaction mechanism. However, the first and second type of effects can also have a strong influence on both the rates and equilibrium yields, as has been shown in several recent theoretical calculations ${ }^{2-20}$ and experimental studies ${ }^{21-36}$.

The effect of the shape of the confining material, in particular, has been the subject of extensive research in connection with zeolite-catalyzed reactions, where the interactions with the confining supramolecular structure of the zeolite have a strong influence on 
determining the allowed reaction pathways $2,4,5,7,8,21,25,26,33$. The terms "shapeselectivity”2,4,8,25,29,35, “inverse shape-selectivity”,4,3,33, “active site shape-selectivity”4,35, and similar terms ${ }^{4,24,26}$ are used frequently in the catalysis literature to describe the ability of zeolites and other supramolecular structures ${ }^{24,27-32}$ to drastically modify the outcome of chemical reactions due to confinement effects. It is, however, quite challenging to elucidate the reasons for these shape-selective effects ${ }^{4,5,24,28}$ because of the complexity of these materials, and the interplay of many different factors that influence experimental results, such as confinement effects on the thermodynamic properties of the substances involved, changes in the diffusion rates among different species in different confining materials, and the details of the supramolecular structure at both the atomic scale and the mesoscale ${ }^{4}$.

Only recently have carbon-based materials received attention for their possible influence on chemical reaction equilibria and dynamics $3,6,9-20,22,34,36$. Graphitic carbon is a suitable material for such studies for several reasons: first, it is a relatively simple material to model, which allows for the exploration of a large parameter space (pore sizes, shapes, etc.) without a very large computational expense; second, it is a remarkably flexible material $^{37-39}$, adopting many different structural forms ${ }^{40}$, which makes it ideal for studying shape-catalytic effects; third, graphitic carbon has a large polarizability, which allows for the study of its influence on reactions through physical interactions using relatively simple models; and lastly, graphitic carbon can be chemically modified in various ways in order to study chemical interactions. Some possibilities of this kind are the inclusion of structural defects in the carbon structure ${ }^{41,42}$, doping ${ }^{37}$ and the addition of functional groups ${ }^{37}$.

In this work, we present a review of our research on some of the different factors that can influence a chemical reaction in confinement through the study of several model systems. We first consider the influence of steric hindrance on the equilibrium and kinetics for the rotational isomerizations of several small hydrocarbons ${ }^{10,11}$. These examples illustrate how 
reaction rates can vary doubly exponentially with the dimensions of the confining material (which we term the "shape-catalytic" effect). As a second example, we consider the unimolecular decomposition of formaldehyde on graphitic carbon pores of various sizes ${ }^{13}$. These results illustrate the influence of electrostatic interactions with the supporting material on the reaction mechanism and equilibrium yield for reactions involving a charge transfer.

As a final example, we consider the interaction of a water molecule with a defective carbon substrate as an example of a chemical interaction that can be enhanced through a shapecatalytic effect. We first show using $a b$ initio calculations how a vacancy site on a graphene surface can induce the thermal splitting of water at relatively low temperatures ${ }^{14}$. We then examine the dissociation on a vacancy site on a nanotube surface, which shows the shape-catalytic effect of the surface curvature. These results are a first step toward the design of catalytic materials that take advantage of different enhancing effects simultaneously.

The rest of this work is organized as follows: In Chapter 2 we discuss some of the basic concepts associated with the molecular-level modeling of chemical reactions and review some of the main methods available for such studies. In Chapter 3 we present our results on the modeling of the isomerization kinetics of hydrocarbons in confinement, which illustrate the importance of the shape-catalytic effect in nanoporous materials. Chapter 4 contains our results on the unimolecular decomposition of formaldehyde, as an example of how physical interactions with the confining material can affect the reaction equilibrium and dynamics. In Chapter 5, we present our studies on the thermal splitting of water over defective carbon substrates. Finally, in Chapter 6 we offer some conclusions regarding the implications of this work and suggest future directions for research in this area. 


\section{References and Notes}

\footnotetext{
${ }^{1}$ Some of the material in this chapter and subsequent chapters has been published in: E.E. Santiso, L. Huang, K.E. Gubbins, M.K. Kostov, A.M. George and Marco Buongiorno Nardelli, “Ab initio simulations of chemical reactions in nanostructured carbon materials”, in Quantum Chemical Calculations of Surfaces and Interfaces of Materials, American Scientific Publishers, Valencia, California (2007).

${ }^{2}$ D. Lesthaeghe, B. De Sterck, V. Van Speybroeck, G.B. Marin, M. Waroquier, Angew. Chem. Int. Ed. 46, 1311 (2007).

${ }^{3}$ K.P. Travis, D.J. Searles, J. Chem. Phys. 125, 164501 (2006).

${ }^{4}$ L.A. Clark, M. Sierka, J. Sauer, J. Am. Chem. Soc. 126, 936 (2004).

${ }^{5}$ M. Schenk, S. Calero, T.L.M. Maesen, L.L. van Benthem, M.G. Verbeek, B. Smit, Angew. Chem. Int. Ed. 41, 2499 (2002).

${ }^{6}$ M.D. Halls, H.B. Schlegel, J. Phys. Chem. B 106, 1921 (2002) .

${ }^{7}$ X. Rozanska, R.A. van Santen, F. Hutschka, J. Hafner, J. Am. Chem. Soc. 123, 7655 (2001).

${ }^{8}$ G. Tasi, I. Pálinkó, F. Mizukami, React. Kinet. Catal. Lett. 74, 317 (2001).

${ }^{9}$ M. Lisal, J.K. Brennan, W.R. Smith, J. Chem. Phys. 124, 064712 (2006).

${ }^{10}$ E.E. Santiso, M. Buongiorno Nardelli, K.E. Gubbins, Adsorption, submitted (2007).

${ }^{11}$ E.E. Santiso, M. Buongiorno Nardelli, K.E. Gubbins, J. Chem. Phys., submitted (2007).

${ }^{12}$ E.E. Santiso, M.K. Kostov, A.M. George, M. Buongiorno Nardelli, K.E. Gubbins, Appl. Surf. Sci. 253, 5570 (2007).

${ }^{13}$ E.E. Santiso, A.M. George, K.E. Gubbins, M. Buongiorno Nardelli, J. Chem. Phys. 125, 084711 (2006).

${ }^{14}$ M.K. Kostov, E.E. Santiso, A.M. George, K.E. Gubbins, M. Buongiorno Nardelli, Phys. Rev. Lett. 95, 136105 (2005).

${ }^{15}$ E.E. Santiso, A.M. George, C.H. Turner, M.K. Kostov, K.E. Gubbins, M. Buongiorno Nardelli, M. Sliwinska-Bartkowiak, Appl. Surf. Sci. 252, 766 (2005).
} 
${ }^{16}$ E.E. Santiso, A.M. George, M. Sliwinska-Bartkowiak, M. Buongiorno Nardelli, K.E. Gubbins, Adsorption 11, 349 (2005).

${ }^{17}$ C.H. Turner, K.E. Gubbins, J. Chem. Phys. 119, 6057 (2003).

${ }^{18}$ C.H. Turner, J.K. Brennan, J.K. Johnson, K.E. Gubbins, J. Chem. Phys. 116, 2138 (2002).

${ }^{19}$ C.H. Turner, J. Pikunic, K.E. Gubbins, Mol. Phys. 99, 1991 (2001).

${ }^{20}$ C.H. Turner, J.K. Johnson, K.E. Gubbins, J. Chem Phys. 114, 1851 (2001).

${ }^{21}$ J.F.M. Denayer, A.R. Ocakoglu, J.A. Martens, G.V. Baron, J. Catal. 226, 240 (2004)

${ }^{22}$ O. Byl, P. Kondratyuk, J.T. Yates, J. Phys. Chem. B 107, 4277 (2003).

${ }^{23}$ C.K. Regan, S.L. Craig, J.I. Brauman, Science 295, 2245 (2002)

${ }^{24}$ E. Brunet, Chirality 14, 135 (2002).

${ }^{25}$ B. Arstad, S. Kolboe, J. Am. Chem. Soc. 123, 8137 (2001).

${ }^{26}$ S. Jayaraman, S. Uppili, A. Natarajan, A. Joy, K.C.W. Chong, M.R. Netherton, A. Zenova, J.R. Scheffer, V. Ramamurthy, Tetrahedron Lett. 41, 8231 (2000).

${ }^{27}$ K.A. Swiss, R.A. Firestone, J. Org. Chem. 64, 2158 (1999).

${ }^{28}$ T. Kunieda, J.-H. Kim, M. Niwa, J. Catal. 188, 431 (1999).

${ }^{29}$ C.W. Jones, K. Tsuji, M.E. Davis, Nature 393, 52 (1998).

${ }^{30}$ J. Kang, J. Santamaría, G. Hilmersson, J. Rebek, Jr., J. Am. Chem. Soc. 120, 7389 (1998).

${ }^{31}$ J. Kang, G. Hilmersson, J. Santamaría, J. Rebek, Jr., J. Am. Chem. Soc. 120, 3650 (1998).

32 J. Kang, J. Rebek, Jr., Nature 382, 239 (1996).

${ }^{33}$ D.S. Santilli, T.V. Harris, S.I. Zones, Microporous Mater. 1, 329 (1993).

${ }^{34}$ J. Imai, M. Souma, S. Ozeki, T. Suzuki, K. Kaneko, J. Phys. Chem. 95, 9955 (1991).

${ }^{35}$ M.W. Anderson, J. Klinowski, J. Am. Chem. Soc. 112, 10 (1990).

${ }^{36}$ K. Kaneko, N. Fukuzaki, K. Kakei, T. Suzuki, S. Ozeki, Langmuir 5, 960 (1989).

${ }^{37}$ M.S. Dresselhaus, G. Dresselhaus, P.C. Eklund, Science of Fullerenes and Carbon Nanotubes, Academic Press, San Diego (1995). 
${ }^{38}$ S. Bandow, F. Kokai, K. Takahashi, M. Yudasaka, L.C. Qin, S. Ijima, Chem. Phys. Lett. 321, 514 (2000).

${ }^{39}$ S. Berber, Y.K. Kwon, D. Tomanek, Phys. Rev. B 62, R2291 (2000).

${ }^{40}$ T.J. Bandosz, M.J. Biggs, K.E. Gubbins, Y. Hattori, T. Iiyama, K. Kaneko, J. Pikunic and K. Thompson, Chem. Phys. Carbon 28, 41 (2003).

${ }^{41}$ A. Hashimoto, K. Suenaga, A. Gloter, K. Urita, S. Iijima, Nature 430, 7002 (2004).

${ }^{42}$ A.A. El-Barbary, Ph.D. Thesis, University of Sussex (2005). 


\section{Chapter 2}

\section{Review of Methods for Modeling Chemical Reactions}

In this chapter, we present a review of the most widely used methods to model chemical reactions, at both the electronic and atomistic levels. While, in principle, ab initio methods alone should provide the required prediction of reaction mechanisms, yields and rates, in practice this can rarely be achieved due to the intensive nature of the computations and the poor scaling of the computational burden with the number of electrons. In many applications a combination of $a b$ initio and semi-classical atomistic simulations will be needed. Specialized atomistic simulation methods are necessary, since the reactions are themselves rare events, and the free energy landscape for the reaction is often rugged with many possible reaction paths. We provide a survey of these methods, with comments on their applicability and a description of their strengths and weaknesses. The material in this chapter has been published in ref. 1

\subsection{Introduction}

The newcomers to the arcane field of modeling of chemical reactions will usually find themselves bewildered by the profusion of proposed methods, both $a b$ initio and classical atomistic. In the case of the $a b$ initio approaches, we find a plethora of mathematical formulations of the electronic problem, approximations, basis sets. Such methods include Hartree-Fock theory, Configuration Interaction, Møller-Plesset perturbation methods, Coupled-Cluster methods, Multireference Self-Consistent Field methods, various SemiEmpirical Methods, Density Functional Theory and Car-Parrinello Molecular Dynamics, and within each of these there are, on average, four or five different variations. The

atomistic methods include various forms of Transition State Theory, "Blue Moon" 
Molecular Dynamics, the Reactive Flux Method, Transition Path Sampling, Kinetic Monte Carlo, Quantum Mechanics/Molecular Mechanics, and others. The multitude of acronyms used, particularly for the quantum mechanical methods, further strengthens the apparent impenetrability of the field. We find ourselves confronted with sentences such as: "Geometries were optimized at the B3LYP/6-31G(d) level and single-point energies were obtained from CASSCF and CASPT2 calculations".

This wide range of methods reflects both the difficulty and importance of this area. While surveys exist of some of the $\mathrm{ab}_{\text {initio }^{2-10}}$ and classical atomistic ${ }^{10-12}$ methods, there does not appear to be a general overview of the important methods of both types. However, many applications require a multi-scale approach in which $a b$ initio and atomistic methods are combined; due to computer limitations, $a b$ initio methods alone are often insufficient to deal with adequate system sizes or time scales. This is particularly the case when strong intermolecular interactions are involved, as in reactions in solution, in supercritical fluids, and in nanostructured environments (reactions in porous media, composites, reverse micelles, etc.). In this chapter we present a review of some of the most important $a b$ initio and classical atomistic methods, at a level that is appropriate for a newcomer to the area. While space does not permit us to review all of the methods that have been proposed, we have tried to select those that we believe are most widely useful, and endeavour to make this area accessible to those researchers who, while being familiar with some simulation or $a b$ initio methods, may be approaching reacting systems for the first time. Examples of recent applications of each of the methods are mentioned at the end of each section.

We begin by introducing the basic concepts common to chemical reactions (Section 2.2), such as potential energy surfaces, transition states and reaction coordinates. Empirical force field methods to describe the potential energy surface, and the associated molecular mechanics and quantum mechanics/molecular mechanics methods are described in Section 2.3. The basis of the $a b$ initio methods, and the various approximate methods available for 
their solution, are covered in Section 2.4. In Section 2.5 we discuss the atomistic simulation methods, and in Section 2.6 we present some concluding remarks.

\subsection{Chemical Reactions $\mathrm{s}^{2-6,13-15}$}

Before we start discussing methods for dealing with chemical reactions, it is convenient to talk about the kind of problems we want to consider and define some terms. From a practical point of view, what we usually want to obtain about a chemical reaction is either an equilibrium constant or a kinetic rate. Equilibrium constants are in this sense the easiest to obtain because they depend only on the thermodynamic properties of the reactants and the products. One may not know how a reaction proceeds from reactants to products and still get the equilibrium constant for the reaction. The complications that may arise are those associated with the estimation of thermodynamic properties: non-ideality, effects due to external imposed fields, etc. In section 2.5 we will talk about a method to obtain

equilibrium constants in such non-ideal conditions, the Reactive Monte Carlo method, which can deal with this kind of situation.

The estimation of rate constants is an entirely different matter, however, because they are dynamic properties and hence depend on how the system evolves from reactants to products. Even for very simple systems, e.g. a reacting mixture of ideal gases, it is not a trivial task to figure this out. Since reactions usually involve breaking and formation of chemical bonds, finding out exactly how a reaction proceeds is inherently a quantum mechanical problem. In sections 2.3 and 2.4 we will discuss some of the methods that can be used to describe these processes.

One of the things that we will discuss in section 4 is the Born-Oppenheimer approximation. Within this approximation, one assumes that the only relevant parameters 
necessary to describe the reaction mechanism are the positions of the atomic nuclei in space. Thus a Potential Energy Surface (PES), which gives the potential energy of the system $E\left(\left\{\mathbf{R}_{I}\right\}\right)$ as a function of the nuclear configuration, is used to describe a chemical reaction.

Figure 2.2.1 is a sketch of a PES. This plot corresponds to a simple case where the PES depends only on two coordinates ${ }^{16}$. The variables $f$ and $g$ represent functions of the configurational variables and could be, for example, distances between two atoms, angles formed by three atoms or dihedral angles formed by four atoms. The labels A and B indicate the reactant and product states. The maximum along the minimum energy path, labeled as TS, is the transition state (also known as the activated state or activated complex). This point is a first-order saddle point, i.e. the energy increases in all directions but one.

The PES for a given reaction can be found using a quantum mechanical method. Its description, however, can be very complicated. In principle, the nuclear configuration space $\left\{\mathbf{R}_{I}\right\}$ contains the coordinates of all the nuclei that may have an effect on the reaction, i.e. not only the reactants and the products but also all the neighboring atoms. In some cases, for example reactions happening in solution or in confinement, there may be collective effects involving large numbers of molecules that affect the reaction ${ }^{17-20}$. Thus the potential energy surface may exist in a highly multidimensional space, and just describing it could be impossible. It is often necessary to simplify the system by considering only some of the coordinates. Even then, or in simpler cases like a reaction occurring in an ideal gas, there may still be several possible pathways through which the reaction can proceed.

At finite temperature, the reaction path is really defined on a free energy surface, and this must be taken into account when calculating reaction rates. However, the way to define 
reaction paths is usually to do $a b$ initio calculations, and these are done at zero temperature (i.e. the atoms are taken as stationary), so temperature effects have to be included afterwards. It is possible to directly explore the free energy surface using, for example, modified $a b$ initio molecular dynamics methods, but these approaches are relatively new and have not yet had widespread application ${ }^{21}$.

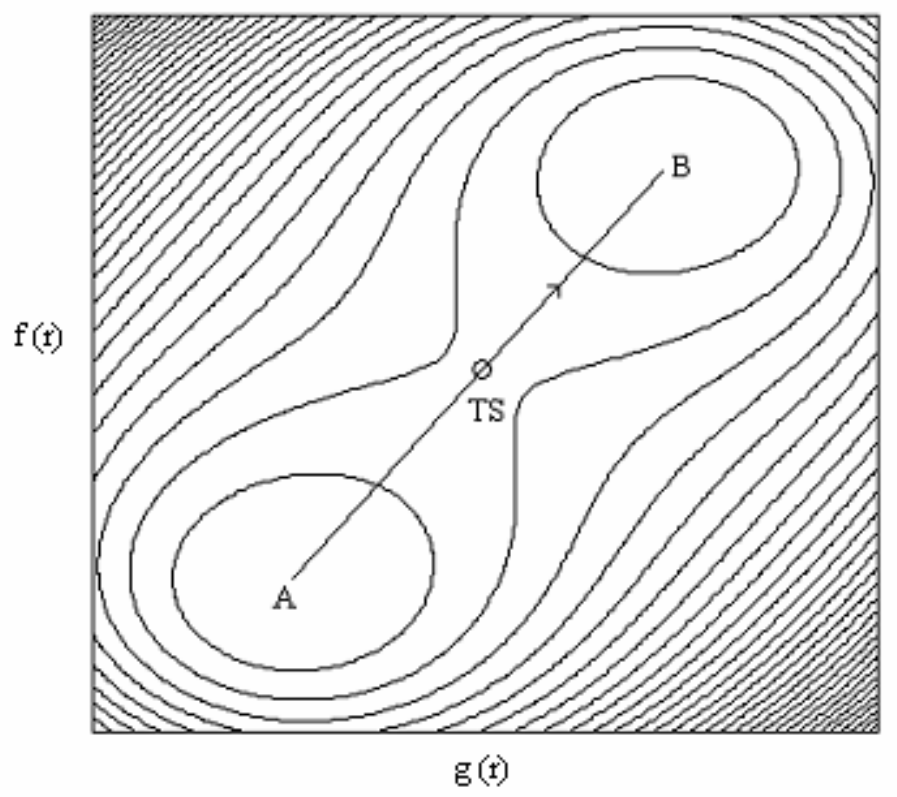

Figure 2.2.1. A simple potential energy surface; contours are lines of constant energy. The variables $f(r)$ and $g(r)$ represent functions of the configuration. A and $B$ are the reactant and product states, and TS is the transition state.

Many methods for estimating reaction rates require defining one or more reaction coordinates that serve as a measure of the extent of the reaction. A reaction coordinate is a function of the configurational variables $\left\{\mathbf{R}_{I}\right\}$ that measures how far along the reaction path the system is in a given configuration. In principle the way to find this function is to search mathematically for the path(s) of minimum energy on the PES that connects the 
reactants to the products (such as the one indicated in figure 2.1). The reaction coordinate(s) can then be defined by parameterizing this path. When this procedure is followed one speaks of an intrinsic reaction coordinate, a term due to K. Fukui ${ }^{22-24}$.

A way to obtain minimum energy paths and hence define intrinsic reaction coordinates is to follow the softest vibrational mode of the reacting molecule ${ }^{22-24}$. This requires numerically calculating the Hessian ${ }^{25}$ of the potential energy surface as one advances along the path, and finding its lowest eigenvalue and corresponding eigenvector, and is usually a very expensive calculation ${ }^{26}$. In some cases there are also several possible pathways, and it would be necessary to move along all of them, which multiplies the computational cost.

In principle, intrinsic reaction coordinates are the correct ones. Nevertheless, obtaining a reaction coordinate in this way is costly, and frequently the parameterized path obtained from a numerical method is difficult to interpret. For this reason, it is often preferable to choose a reaction coordinate beforehand based on an intuition of how the reaction should proceed, or on available data. By choosing a reaction coordinate in this way, however, one is artificially imposing a reaction path. If this path turns out to be very different from the true minimum energy path, the results obtained from it (e.g. reaction rates) may be far off and even physically inconsistent. There are several ways to check whether one has chosen a good reaction coordinate besides, of course, doing the full search for the minimum energy path. Some of these will be discussed in section 2.5. Choosing the right reaction coordinate(s) for complex systems such as reactions occurring in solvents or involving macromolecules is one of the major problems in the simulation of chemical reactions. In many cases it is a trial-and-error procedure, and one of the standing problems in the field is to find a systematical and practical way to deal with this.

The ideas above are best illustrated with an example. Figure 2.2.2 shows a contour plot of the collinear potential energy surface for the reaction $\mathrm{O}+\mathrm{H}_{2} \rightarrow \mathrm{H}+\mathrm{OH}$ as obtained by 
Schinke and Lester" 27 . The points labeled " $\mathrm{O}+\mathrm{H}_{2}$ " and " $\mathrm{H}+\mathrm{OH}$ " correspond to reactants and products states. The line joining them is the minimum energy path, i.e. the most probable reaction path, and the point labeled "TS" is the transition state, the first-order saddle point along the reaction path. A 3D plot showing the corresponding values of the energy is in figure 2.2.3.

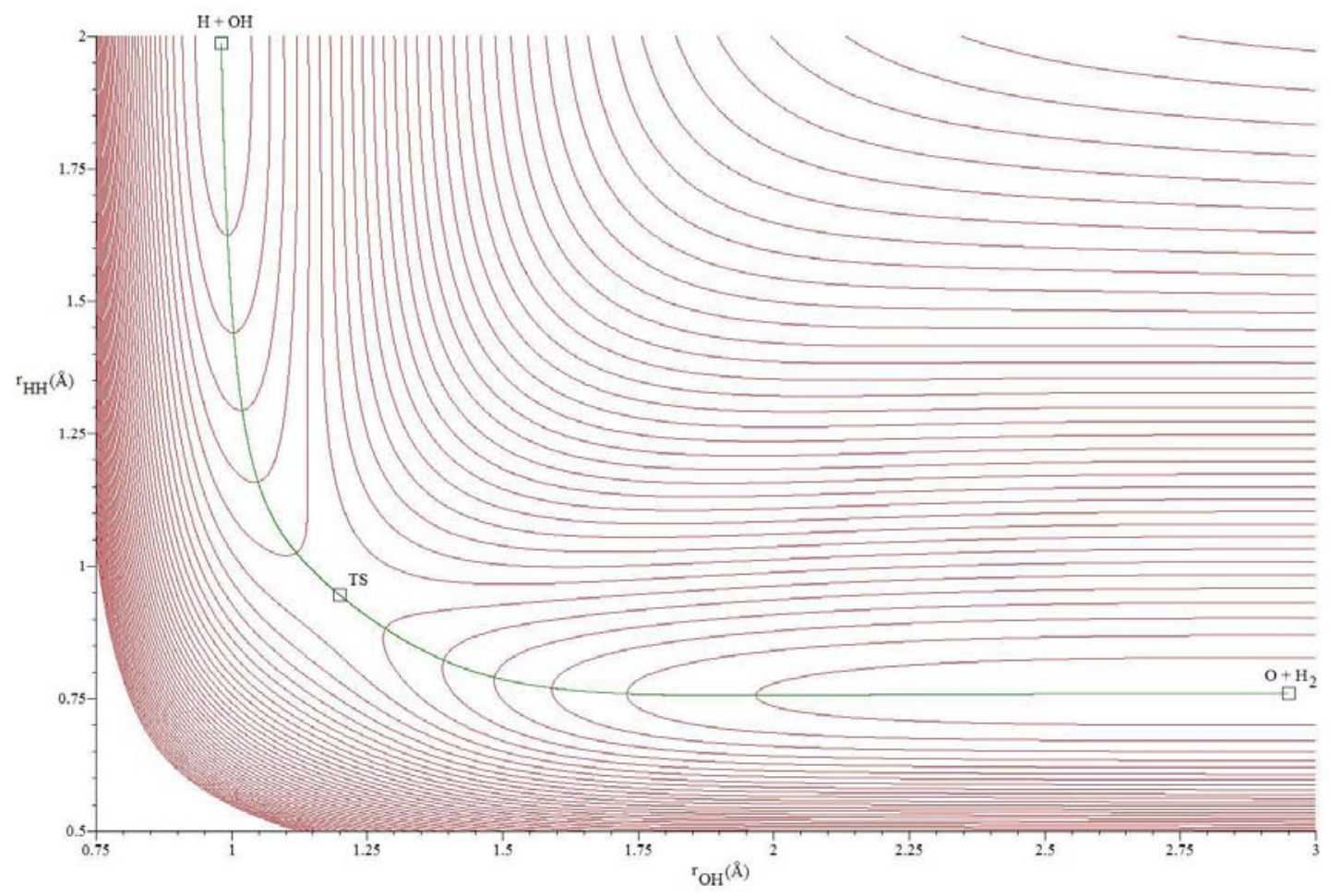

Figure 2.2.2. Contour plot of the potential energy surface for the reaction $\mathrm{O}+\mathrm{H}_{2} \rightarrow$ $\mathrm{H}+\mathrm{OH}$ as obtained with the data in ref. 27. The green line is the minimum energy path.

The reaction path shown in figure 2.2.2 was obtained by following the softest eigenmode of the Hessian and minimizing along all other directions (in this case one), i.e. it corresponds to an intrinsic reaction coordinate. Since the computational cost of calculating 
the Hessian does not always permit using this definition, one may need to rely on intuition about the reaction mechanism to define the reaction coordinate.

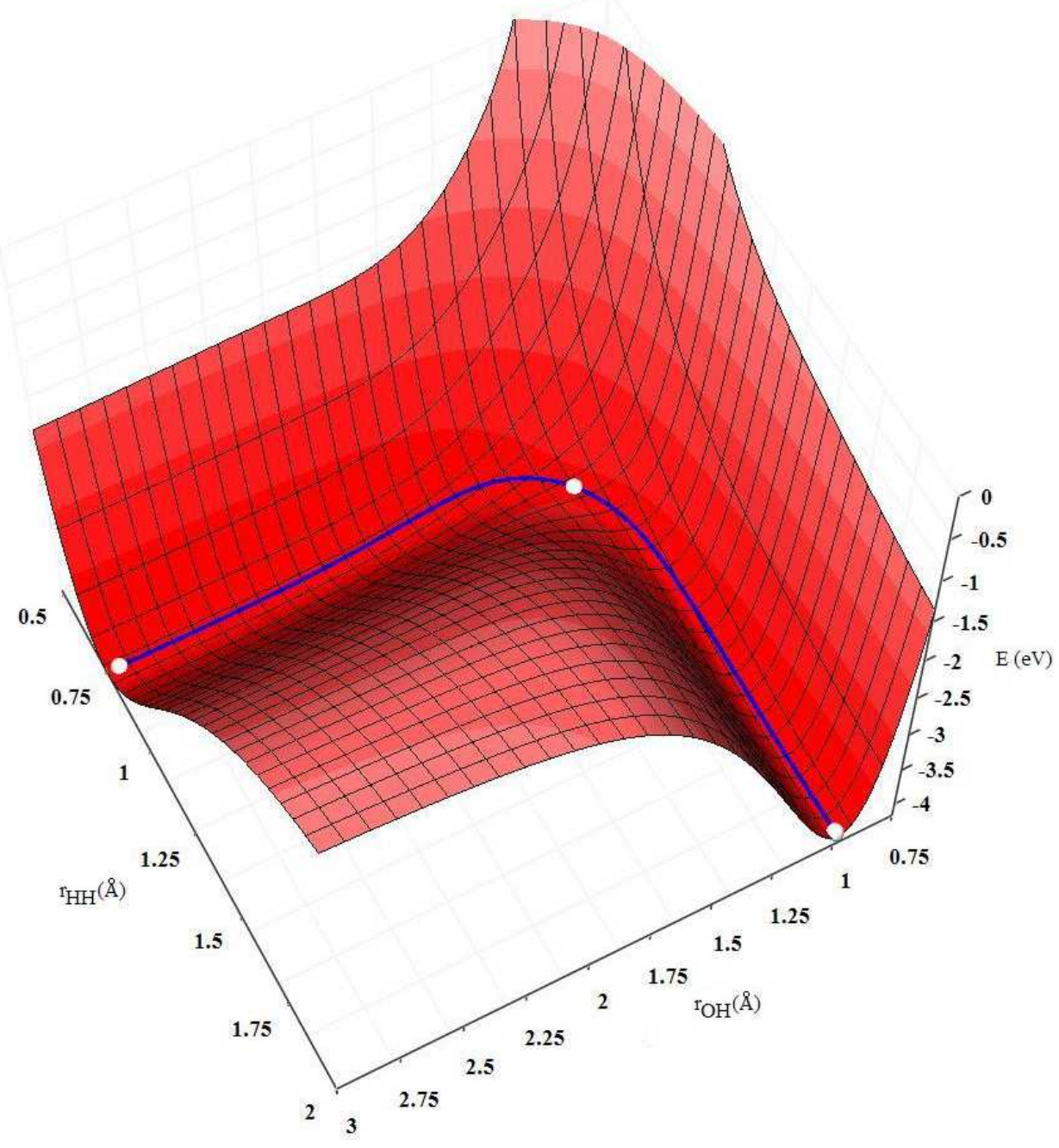

Figure 2.2.3 Potential energy surface for $\mathrm{O}+\mathrm{H}_{2} \rightarrow \mathrm{H}+\mathrm{OH}$. The thick line is the minimum energy path.

Let us imagine that, for the reaction above, we choose to define our reaction coordinate as the distance between the two hydrogen atoms. With this definition, the lines of constant 
reaction coordinate will be horizontal lines on the contour plot. The points on the reaction path will then be the minima of the energy along these lines, as shown in figure 2.2.4. From this figure we see that the reaction path obtained using this definition of the reaction coordinate is very different from the minimum energy path. Furthermore, starting from the products side and moving back we get a different path, with a large hysteresis loop. As discussed in section 2.5.3, this is one way to identify an inadequate choice of reaction coordinate.

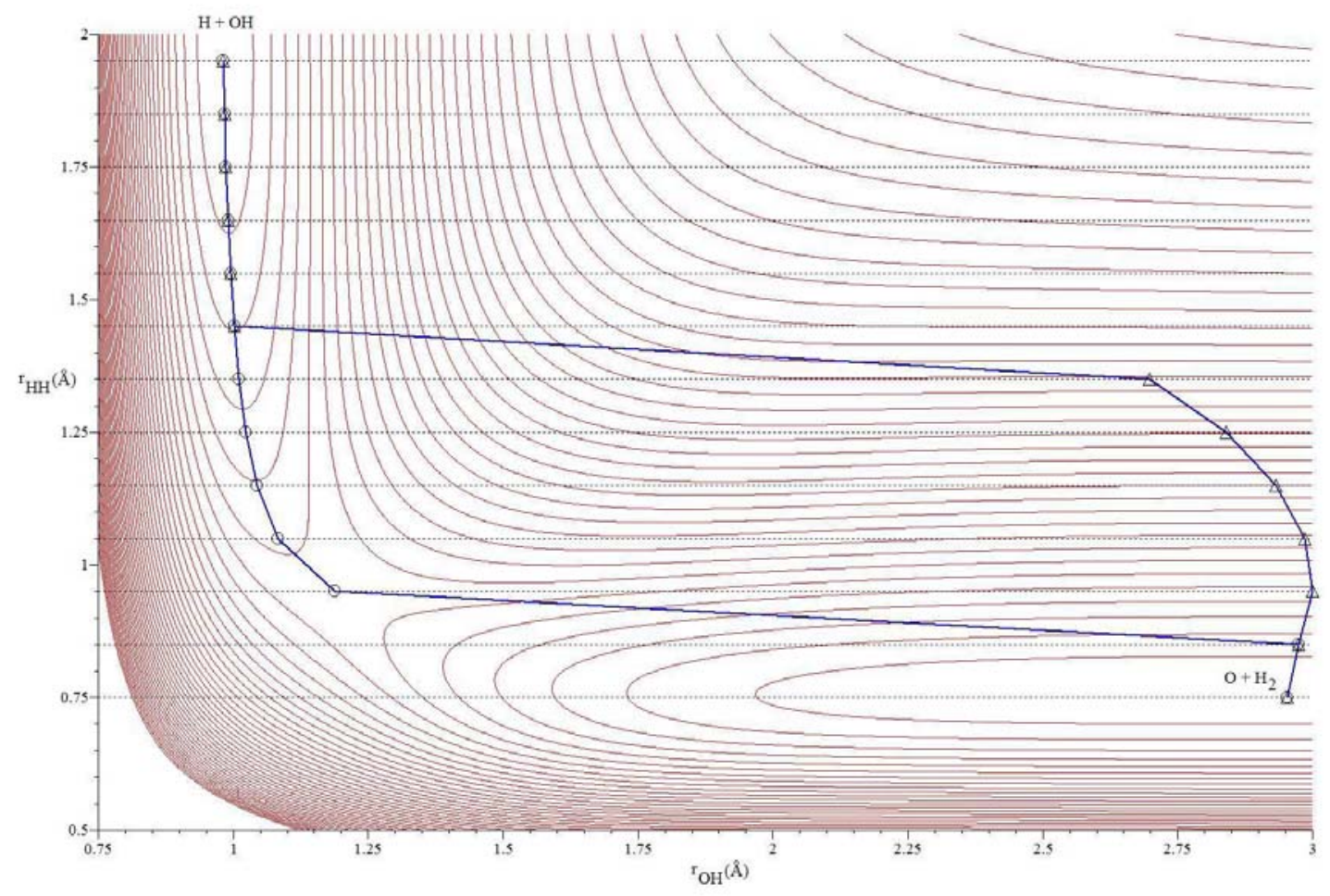

Figure 2.2.4. The reaction path using the distance $H-H$ as a reaction coordinate. The dashed horizontal lines correspond to a constant reaction coordinate. The triangles are the minimum energy points obtained in the forward direction, and the circles are the points obtained in the backwards direction. 
If we choose instead to use the difference between the $\mathrm{H}-\mathrm{H}$ and the $\mathrm{O}-\mathrm{H}$ distance, $\mathrm{r}_{\mathrm{HH}}-\mathrm{r}_{\mathrm{OH}}$, as our reaction coordinate, the results are quite different. The lines of constant reaction coordinate are now inclined $45^{\circ}$ with respect to the horizontal, and minimizing the energy along these lines gives the path shown in figure 2.2.5. This path is much closer to the minimum energy path than the one shown in figure 2.2.4. Furthermore, the reaction path is the same going forwards and backwards, i.e. there is no hysteresis as in figure 2.2.4. All of this indicates that this is a good choice of reaction coordinate.

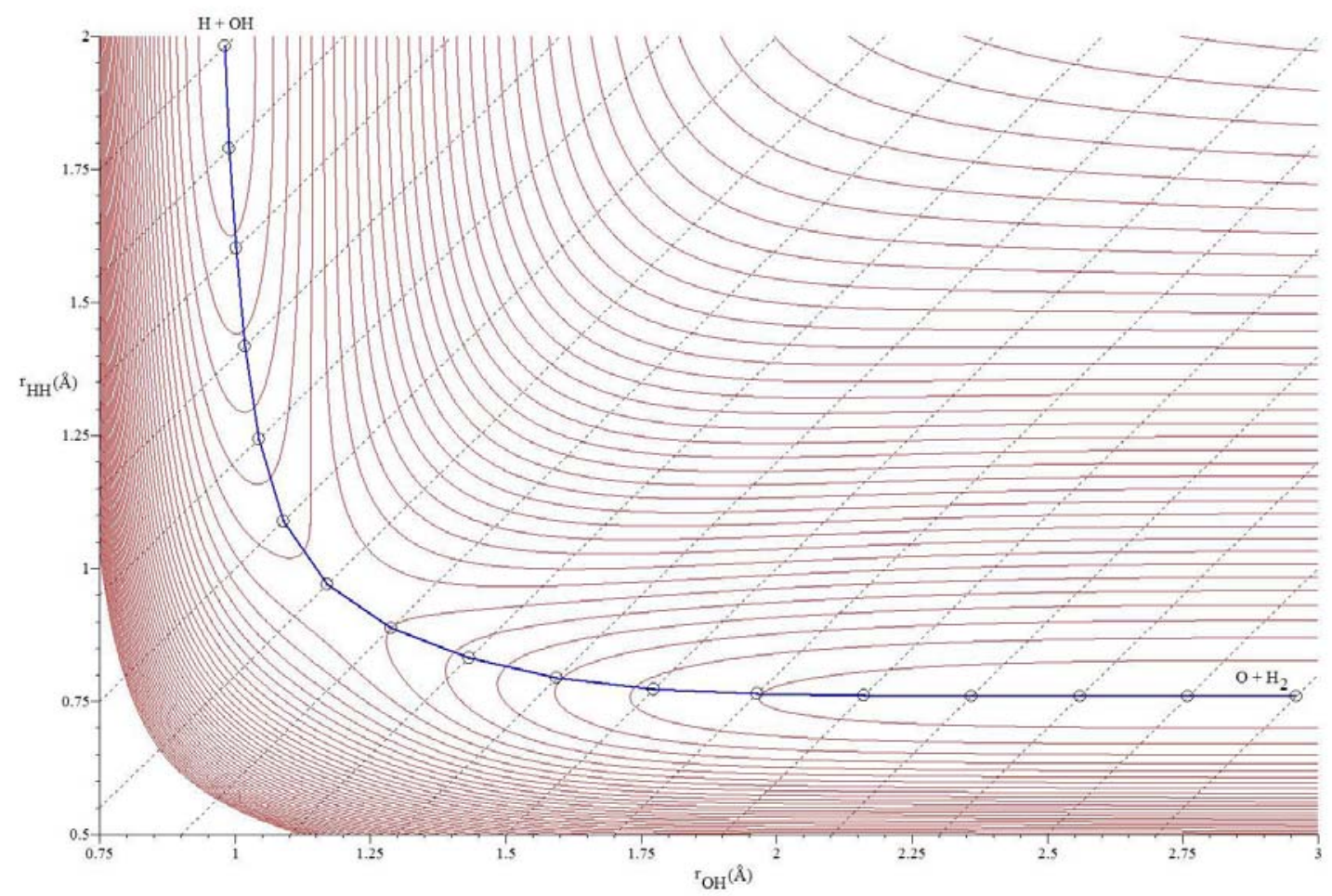

Figure 2.2.5. The reaction path using the difference between the $\mathrm{H}-\mathrm{H}$ and $\mathrm{O}-\mathrm{H}$ distances as a reaction coordinate. The dashed diagonal lines correspond to a constant reaction coordinate. The circles are the minimum energy points found on the lines of constant reaction coordinate. 
Even when using a simple method, like transition state theory, which does not require determining the full minimum energy path, estimating a reaction rate requires at least finding the minima and the transition state(s) in the $\mathrm{PES}^{28}$. There are standard optimization methods for dealing with this (see, for example, refs. 29-31). Quasi-Newton methods, particularly the Broyden ${ }^{32,33}$-Fletcher ${ }^{34}$-Goldfarb ${ }^{35}$-Shanno ${ }^{36}$ (BFGS) method are generally considered the best for this kind of problem. The BFGS method works only for a minimization problem, i.e. for the location of the reactant and product states. There are also quasi-Newton methods that can be used to find first order saddle points, such as the RFO (Rational Function Optimization) and PRFO (Partitioned Rational Function Optimization) methods $^{37}$. For large systems, a limited-memory BFGS method ${ }^{38,39}$ that does not require storing the full Hessian is available. We will not describe these methods in detail in this work. Schlegel ${ }^{31}$ has recently published a review of the methods used for exploring PESs, and a good introduction can also be found in ref. 4. There are also methods available to efficiently construct a PES by interpolating the results from a subset of points, either by assuming a pre-specified functional form ${ }^{40}$, or using numerical interpolation techniques ${ }^{41-}$ 43 , including iterative improving of the PES by using results from rate calculations ${ }^{44-50}$ or optimization of discretized reaction paths ${ }^{51-53}$. Once an interpolated PES is known, it is much easier to determine its main features. Finally, there are newer methods that can be used to obtain the minimum energy path on the PES for given initial and final states without computing second derivatives of the energy, like the nudged elastic band method of Jónsson et al. ${ }^{54}$. Since these methods do not require obtaining the Hessian, they can be used to treat more complex systems.

The description of chemical reactions in terms of reactants, products and a transition state, although simple, is very useful. But it is necessary to keep in mind that for many reactions there is not just a single transition state or reaction path. In many cases there are intermediate stable complexes, and multiple energy barriers, connecting the reactant and product sides. Choosing a suitable method for predicting reaction rates usually requires a 
previous understanding of the reaction mechanism. It is also important to know the size of the energy barrier(s) that the system has to overcome for the reaction to occur. For most reactions, energy barriers are large and reactions happen on a time scale much longer than that characteristic of the molecular motion. If such reactions proceed in a way such that there is good thermal coupling between the transition state molecules and the remaining molecules, and there are only one or a few simple mechanisms for the reaction to proceed, it is possible to use multi-scale classical trajectory methods such as the reactive flux method or blue-moon molecular dynamics (discussed in section 2.5). If this is not the case, as for example in photochemical reactions, a fully quantum mechanical description of the dynamics is necessary, as in the wave packet propagation methods. A classical trajectory description is also possible for systems with complex PES's, where there is not a simple reaction mechanism, at a higher computational cost. This is the case for the transition path sampling method, also discussed in section 2.5.

\subsection{Force Field Methods.}

Chemical reactions involve the breaking and formation of bonds, which can in principle only be described correctly using quantum mechanics. For the description of many complex systems, however, it is not practical to perform quantum mechanical calculations at each configuration to determine the potential energies. An alternative to this is to construct empirical equations, based on quantum mechanical calculations or experimental results (or both), that describe the PES approximately. These methods are usually called empirical force field (FF) methods. The exploration of PESs using empirical force fields is often referred to as molecular mechanics (MM). In this section we describe the main ideas behind the FF approach, and also briefly discuss the combination of FF and electronic structure methods. 


\subsubsection{Semiempirical Force Fields ${ }^{4,555-58}$.}

A feature common to FF methods is the decomposition of the total potential energy into several components. A typical FF expansion of the energy is of the form:

$$
E_{\text {total }}\left(\left\{\mathbf{R}_{I}\right\}\right)=E_{\text {bond }}+E_{\text {val }}+E_{\text {tors }}+E_{\text {vdW }}+E_{\text {coul }}+E_{\text {corr }}
$$

In equation (2.3.1), $E_{\text {total }}\left(\left\{\mathbf{R}_{I}\right\}\right)$ represents the total energy as a function of the nuclear configuration, i.e. the PES. Each of the terms is also dependent on the nuclear configuration, but this dependence is not written explicitly for the sake of brevity.

The first three and the last term in (2.3.1) are associated with intramolecular interactions: $E_{\text {bond }}$ is the energy associated with stretching/compressing bonds between two atoms, $E_{v a l}$ is the energy associated with the bending of valence angles, $E_{\text {tors }}$ is the torsional energy for rotations around bonds. $E_{\text {corr }}$ is a correction term that can include contributions due to conjugation, cross interactions, out-of-plane bending, over/under coordination, etc.

The fourth and fifth terms in (2.3.1) represent the intermolecular interactions: $E_{v d W}$ is the van der Waals contribution and $E_{\text {coul }}$ is the electrostatic contribution. Figure 2.3.1 shows a schematic of what each of the terms accounts for. In some FFs the nuclear configuration is simplified by using the united atom approach, which models groups of atoms (e.g. $\mathrm{CH}_{3}$, $\mathrm{CH}_{2}$, etc.) as a single atom to reduce the computational cost. 

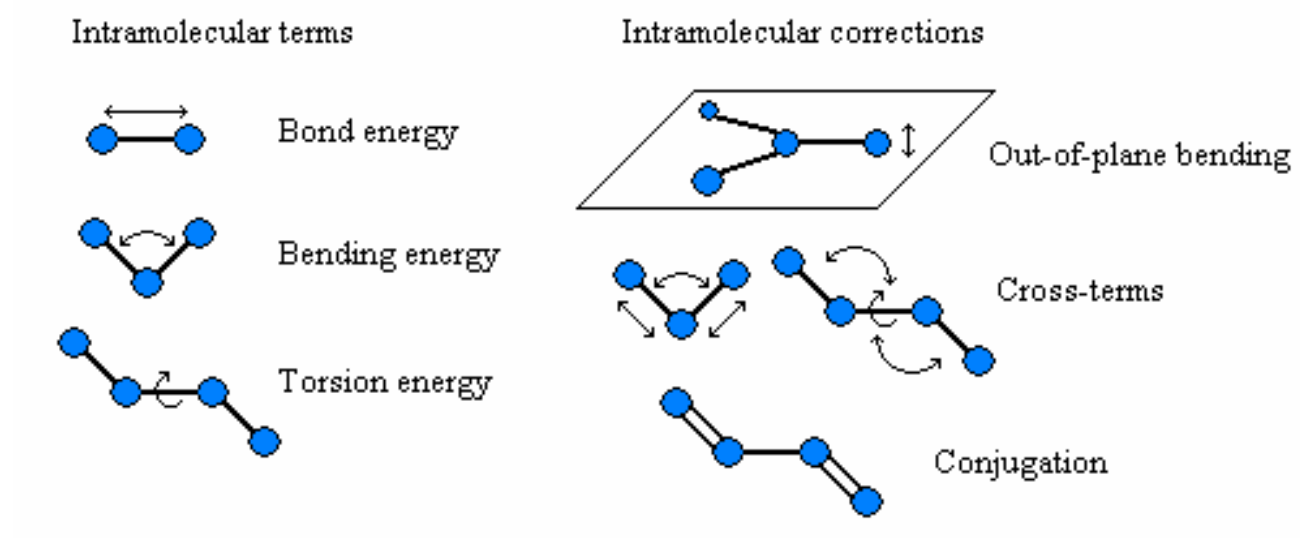

Intermolecular terms
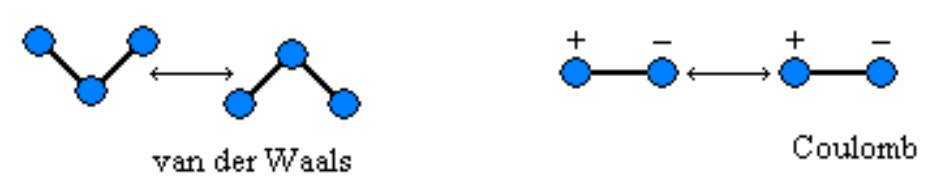

Figure 2.3.1. Energy contributions in a typical force field.

There are different ways to represent each of the energy contributions in (2.3.1), depending on the particular FF method used. The simplest way to represent the energies $E_{\text {bond }}$ and $E_{v a l}$, for example, is the harmonic approximation:

$$
\begin{aligned}
& E_{\text {bond }, I J}=k_{\text {bond }, I J}\left(R_{I J}-R_{I J}^{0}\right)^{2} \\
& E_{\text {val }, I J K}=k_{\text {val }, I J K}\left(\theta_{I J K}-\theta_{I J K}^{0}\right)^{2}
\end{aligned}
$$

In (2.3.2) and (2.3.3), $E_{b o n d, I J}$ and $E_{\text {val,IJK }}$ are the stretching energy associated with the bond between the nuclei $I$ and $J$ and the bending energy associated with the valence angle between the nuclei $I, J$ and $K . R_{I J}$ and $\theta_{I J K}$ are the distance between $I$ and $J$ and the valence angle between $I, J$ and $K ; R_{I J}^{0}$ and $\theta_{I J K}^{0}$ are the corresponding equilibrium values 
of these quantities. The force constants $k_{b o n d, I J}$ and $k_{v a l, I J K}$ are empirical parameters that must be fitted to available data. The $E_{b o n d, I J}$ and $E_{\text {val,IJK }}$ values would have to be summed over all bonds and valence angles to give the $E_{\text {bond }}$ and $E_{\text {val }}$ terms in $(2.3 .1)^{59}$. The harmonic approximation has the advantage of its very low computational cost, but it will only work well for small deviations from the equilibrium values. It is possible to add higher-order corrections (typically up to fourth order) to (2.3.2)-(2.3.3) to get more accurate results at low separations, but this requires additional parameters. These Taylor expansions will also not work when the FF is used to describe a dissociation, since the energy will diverge as the separation grows. In order to describe the dissociation limit correctly different functional forms must be used. A typical choice is the Morse ${ }^{60}$ potential:

$$
E_{\text {bond }, I J}=D\left[1-\exp \left(a R_{I J}\right)\right]^{2}
$$

In (2.3.4), $D$ is the dissociation energy and $a$ is a parameter related to the classical vibrational frequency of the bond. This function describes the stretching energy much more realistically than a Taylor expansion, but has a larger computational cost due to the exponential function. More complicated expressions can be used to fit the intramolecular contribution (for an example see ref. 61).

Torsional contributions to the potential energy are typically represented by Fourier cosine expansions:

$$
E_{\text {tors }, I J K L}=\sum_{n=0}^{N_{F}} a_{n, I J K L} \cos \left(n \omega_{I J K L}\right)
$$

In (2.3.5), $E_{\text {tors,IJKL }}$ is the torsional energy associated with the nuclei $I, J, K$ and $L, N_{F}+1$ is the number of terms in the Fourier expansion, and the $a_{n, I J K L}$ are coefficients that must 
be fitted to available data. These coefficients depend not only on the nature of the atoms, but also on the nature of the bond: a double bond, for example, will have a much higher penalty for rotation than a single bond ${ }^{62}$. The torsional angle $\omega_{I J K L}$ is defined as the angle between the plane containing the nuclei $I, J, K$ and the plane containing the nuclei $J, K, L$.

The correction term $E_{\text {corr }}$ is the one that varies the most between different empirical FFs. It can contain contributions due to conjugation, out-of-plane bending, over/under coordination, coupling between stretching, bending and torsional modes, etc. The particular form of the corrections depends on the kinds of systems that the FF should be able to represent accurately, and the degree of approximation required. The terms $E_{v d w}$ and $E_{\text {coul }}$ account for the non-bonded interactions between the atoms. The van der Waals energy $E_{v d W}$ is often represented as a sum of two contributions: a repulsive part accounting for the overlap energy when the nuclei are too close to each other, and an attractive part due to the mutually induced multipole interactions:

$$
E_{v d W, I J}=E_{o v}\left(R_{I J}\right)-E_{a t t}\left(R_{I J}\right)
$$

The attractive energy $E_{\text {att }}$ in (2.3.6) can in general be expressed as:

$$
E_{a t t}\left(R_{I J}\right)=\frac{C_{6, I J}}{R_{I J}^{6}}+\frac{C_{8, I J}}{R_{I J}^{8}}+\frac{C_{10, I J}}{R_{I J}^{10}}+\cdots
$$

The first term in (2.3.7) accounts for the mutually induced dipole interaction, the second for the induced dipole-induced quadrupole interaction, and so on. The expansion is typically truncated at the first term. 
The overlap energy $E_{o v}$ is more difficult to represent than the attractive part. Although the expansion (2.3.7) can be formally derived using quantum mechanics ${ }^{63-66}$, there is no equivalent formal result for the overlap energy. The form for $E_{o v}$ that has the best theoretical justification is an exponential function. Combining this with the expansion (2.3.7) truncated to the first term we have the Buckingham potential ${ }^{67}$ :

$$
E_{v d W, I J}=A_{I J} \exp \left(-\frac{R_{I J}}{\sigma_{I J}}\right)-C_{I J}\left(\frac{\sigma_{I J}}{r_{I J}}\right)^{6}
$$

The energy parameters $A_{I J}$ and $C_{I J}$, as well as the distance parameter $\sigma_{I J}$ in (2.3.8) are empirical constants. This functional form has the disadvantage of requiring an exponential evaluation. An option is to represent the overlap term by a simpler function of the form $k R_{I J}^{-n}$, where $k$ and $n>6$ are also empirical parameters. The most popular choice of this type is the Lennard-Jones (LJ) potential:

$$
E_{v d W, I J}=4 \varepsilon_{I J}\left[\left(\frac{\sigma_{I J}}{R_{I J}}\right)^{12}-\left(\frac{\sigma_{I J}}{R_{I J}}\right)^{6}\right]
$$

The energy parameter $\varepsilon_{I J}$ and the distance parameter $\sigma_{I J}$ in (2.3.9) are empirical constants. An advantage of the particular form of the LJ potential is that the first term in the square bracket can be computed by multiplying the second one by itself, adding only one multiplication to the computational cost instead of an exponential evaluation. The LJ and Buckingham potentials are the most commonly used for the van der Waals energy. Variations of the LJ equation with different exponents are also common. A Morse or Morse-like potential has also been used to describe the van der Waals energy, as in the $\mathrm{COSMIC}^{68}$ and ReaxFF ${ }^{61,69}$ force fields. 
The electrostatic contribution $E_{\text {coul }}$ to the potential energy can be obtained by assigning charges to atoms and/or bonds in the molecule, or by using a multipole expansion. The latter option requires knowledge of the dipole moment of each molecule (and higher moments if more terms in the multipole expansion are included). The atomic charges used to calculate $E_{\text {coul }}$ can be treated as fitting parameters or obtained using an estimation method. Examples of such methods are the Qeq method ${ }^{70}$ and the electronegativity equalization method $(\mathrm{EEM})^{71,72}$. Sometimes charges are assigned to positions in the molecule that do not correspond to atoms or bonds to improve the fitting, as in some of the potentials used to simulate liquid water. For a description of these see ref. 4.

The main advantage of FF methods in general is their much lower computational cost compared to quantum mechanical calculations. For many complex systems, such as biomolecules and many reactions in solution, FF methods are the only viable alternative to describe the PES. There are many different FFs in the literature. In most cases, the FF is developed to treat a particular class of systems, although there are several FFs that are general (i.e. parameters are available for all atoms). Some commonly used FFs are $^{73}$ the molecular mechanics (MM) force fields of Allinger et al., MM2 $2^{74,75}, \mathrm{MM}^{75-78}$, and MM4 ${ }^{79-83}$, the Merck molecular force field $(\mathrm{MMFF})^{84-88}, \mathrm{AMBER}^{89-91}$ (Assisted Model Building with Energy Refinement), OPLS ${ }^{91-93}$ (Optimized Potentials for Liquid Simulation), CHARMM ${ }^{91,94,95}$ (Chemistry at HARvard Macromolecular Mechanics), GROMOS $^{96-98}$ (GROningen MOlecular Simulation), ECEPP ${ }^{99-101}$ (Empirical Conformational Energy Program for Peptides), and DREIDING ${ }^{102}$. Most of these methods were originally developed for the study of biomolecules such as proteins, nucleic acids and carbohydrates, or hydrocarbons, but some have been extended to handle any atom types. There are also force fields, such as MOMEC ${ }^{103}$ and SHAPES ${ }^{104}$ that were developed for the study of inorganic compounds, particularly transition metal complexes; and force fields specifically developed for hydrocarbon compounds, such as EFF (Empirical Force Field) ${ }^{105,106}$, the Brenner potential ${ }^{107}$, AIREBO (Adaptive Intermolecular Reactive 
Empirical Bond Order) ${ }^{108}$ and ReaxFF ${ }^{61}$. Other more specialized force fields have been reviewed by Leach ${ }^{4}$.

Many force fields, as mentioned in the previous paragraph, have been extended to include practically all kinds of atoms. There are also force fields developed from the beginning with the purpose of spanning the full periodic table, such as UFF (Universal Force Field) ${ }^{109}$ and RFF (Reaction Force Field) ${ }^{110}$. The problem with the more general FFs is that they are in general less accurate than FFs developed specifically for a particular family of compounds, but they have also been around for a shorter time, so it is quite possible that further refinements can make them competitive. The main problem in fitting a general force field is to ensure its transferability while using a reasonable number of parameters; in order to be useful the FF has to be able to predict correctly properties for compounds that fall outside the set used to fit the parameters.

There are several techniques for exploring PESs, and particularly for finding transition states, using FF methods ${ }^{5,111-115}$. These methods are useful for situations where a more accurate description of the system is not possible, and also for finding initial estimates of transition state structures to be further refined with, e.g. an $a b$ initio method. There are also methods that use a combination of a FF with quantum mechanical calculations for efficient exploration of reactive PESs, like the multiconfigurational molecular mechanics (MCMM) $\operatorname{method}^{116,117}$ and the QM- guided molecular mechanics (Q2MM) method ${ }^{118}$, among others ${ }^{119-122}$. Some of these methods are related to the approach discussed in the following section.

The main disadvantage of the FF approach is that there is no way to systematically improve the results within the method. In the case of $a b$ initio methods, for example, one can improve the results by increasing the basis set size, or by using a higher level of theory. In FF this is not possible. The only way to assess the results is to compare to either 
experimental data or to results obtained with an $a b$ initio method, but this comparison is not always possible. Nevertheless, since for many systems a FF approach is practically the only viable option, they remain a very useful tool in computational chemistry.

Another problem with FF methods is that there seem to be too many of them. It is difficult to decide a priori which FF is the best for the particular system that one wants to study. Many comparisons and evaluations of FFs for different applications, however, have been published that can serve as a guide in making such a decision. Some of them can be found in refs.123-137.

Applications of FF methods are numerous, particularly in the study of complex systems like biomolecules, polymers and reactions in solution. Some recent representative works that show the applicability of the FF approach include:

- A molecular dynamics study of seven different oligosaccharide complexes of the

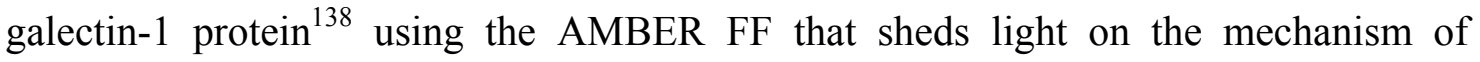
carbohydrate binding in this protein.

- A study of the side-chain conformations in the gramicidin-A channel ${ }^{139}$ through molecular dynamics simulations using the CHARMM FF, showing the different states and the frequency of the transitions between states for this system.

- A conformational study of the O-specific polysaccharide of the bacteria Shigella flexneri $^{140}$, a molecule that plays an important role on the immune response against this microorganism. The study used the MM3 force field, and the models obtained were in good agreement with NMR measurements.

- A study of the dynamical effects of mutations on an RNA hairpin ${ }^{141}$ using constrained molecular dynamics and the free energy perturbation method. The simulations were performed using the CHARMM27 FF. The results obtained show good agreement with experimental measurements. 


\subsection{Ab Initio Methods ${ }^{3-10,142,143}$}

Although one may use a previously fitted force field to describe the PES, obtaining it for a particular system is the purpose of $a b$ initio methods. In these methods the potential energy is obtained using the principles of quantum mechanics, using only as input the nature of the atoms comprising the system.

Since we are restricting ourselves to the calculation of chemical reaction rates using classical trajectory methods, we will not discuss here time-dependent $a b$ initio methods or quantum scattering methods. An introduction to the former can be found in ref. 3, and a recent review in ref. 144. A recent review of the quantum scattering methods is in ref. 145. The quantum Monte Carlo method, which uses the analogy between the time-dependent Schrödinger equation in imaginary time and the classical diffusion equation for electronic structure calculations, will also not be discussed here. Good introductions to this subject can be found in refs. 146 and 147.

The $a b$ initio methods that we will discuss attempt to find approximate solutions to the time-independent Schrödinger equation for a given many-atom system:

$$
\hat{H} \Phi=E \Phi
$$

In (2.4.1), $\Phi$ represents the wavefunction of the many-atom system, $E$ is the total energy, and $\hat{H}$ is the Hamiltonian operator. For the general case of a system of $N$ electrons and $M$ nuclei, the Hamiltonian operator is:

$$
\hat{H}=-\sum_{i=1}^{N} \frac{1}{2} \nabla_{i}^{2}-\sum_{I=1}^{M} \frac{1}{2 M_{I}} \nabla_{I}^{2}-\sum_{i=1}^{N} \sum_{I=1}^{M} \frac{Z_{I}}{r_{i I}}+\sum_{i=1}^{N-1} \sum_{j=i+1}^{N} \frac{1}{r_{i j}}+\sum_{I=1}^{M-1} \sum_{J=I+1}^{M} \frac{Z_{I} Z_{J}}{R_{I J}}
$$


In writing (2.4.2), and in what follows, we use the indices $i, j, \cdots$ for the electrons and the indices $I, J, \cdots$ for the nuclei. The symbol $\nabla_{i}^{2}$ is a Laplacian operator with respect to the coordinates of electron $i$, and $\nabla_{I}^{2}$ is a Laplacian operator with respect to the coordinates of the nucleus $I$. The first term in (2.4.2) represents the electronic kinetic energy; the second term is the nuclear kinetic energy. The remaining three terms are the Coulomb interactions between the nuclei and the electrons, between electron pairs and between nucleus pairs respectively. All the equations we will write in section 2.4 will be in atomic units.

In sections 2.4.1 to 2.4.3 we will introduce some of the basic elements that are common to most $a b$ initio methods: the Born-Oppenheimer approximation, the variational principle and the concept of orbitals and the basis sets used to construct them. In the remaining sections we will explore some of the most common approaches to finding approximate solutions to (2.4.1), and discuss their advantages and limitations.

\subsubsection{The Born-Oppenheimer Approximation.}

Most ab initio methods make use of the Born-Oppenheimer (BO) approximation, proposed by Born and Oppenheimer in $1927^{148,149}$. The idea behind this approximation is that, since the nuclei are much heavier than the electrons, their motion will be much slower, i.e. the characteristic time scales of processes involving the electrons are much smaller. If this is true, the electronic wavefunctions can be found by assuming that the nuclei are static. This means that the PES will only depend on configurational variables, i.e. on the way the nuclei are arranged in space, and not on their momenta. The BO approximation is good for many quantum chemistry problems ${ }^{150}$, but it fails if two different solutions to the electronic Schrödinger equation are very close in energy. If non-BO effects (also called nonadiabatic $^{151}$ effects) may be important, it is possible to include them as corrections after a $\mathrm{BO}$ calculation has been made ${ }^{150}$, e.g. with a perturbation approach. It is also possible to do 
simulations where there is more than one adiabatic ${ }^{151}$ potential energy surface, and the system is permitted to "hop" from one to the other in order to allow for electronic transitions. These methods are called surface-hopping methods ${ }^{152-154}$. For a review on these methods see ref. 155. A review on non-BO reaction dynamics is given in ref. 156.

Within the BO approximation, only the terms in the Hamiltonian (2.4.2) involving the electrons have to be taken into account. The second term -the kinetic energy of the nucleiis neglected because the nuclei are assumed to be static. The last term -the Coulomb repulsion between the nuclei- is assumed to be constant, and thus can be added to the energy after solving the electronic problem. The Hamiltonian for the electronic problem is hence:

$$
\hat{H}_{e}=-\sum_{i=1}^{N} \frac{1}{2} \nabla_{i}^{2}-\sum_{i=1}^{N} \sum_{I=1}^{M} \frac{Z_{I}}{r_{i I}}+\sum_{i=1}^{N-1} \sum_{j=i+1}^{N} \frac{1}{r_{i j}}
$$

where the subscript $e$ refers to the electrons. The corresponding Schrödinger equation for the electronic problem is:

$$
\hat{H}_{e} \Phi_{e}=E_{e}\left(\left\{\mathbf{R}_{I}\right\}\right) \Phi_{e}
$$

The electronic energy $E_{e}$ obtained by solving (2.4.4) depends parametrically on the positions of the nuclei, and it does not include the Coulomb repulsion between them. In order to obtain the total energy of the system it is necessary to add this contribution separately:

$$
E_{\text {total }}\left(\left\{\mathbf{R}_{I}\right\}\right)=E_{e}\left(\left\{\mathbf{R}_{I}\right\}\right)+\sum_{I=1}^{M-1} \sum_{J=A+1}^{M} \frac{Z_{I} Z_{J}}{R_{I J}}
$$


The quantity $E_{\text {total }}\left(\left\{\mathbf{R}_{I}\right\}\right)$ is the PES that is required to study chemical reactions. Within the BO approximation we need to find solutions to the electronic problem (2.4.4), and then use (2.4.5) to get the PES. Note that solving (2.4.4) is an eigenvalue problem that will have an infinite number of solutions. In many cases one is interested in the solution with the lowest energy, which corresponds to the ground state of the electronic system.

\subsubsection{The Variational Principle.}

Many $a b$ initio methods are based on the variational principle, sometimes called the Rayleigh-Ritz variational principle. This principle states that, given any normalized electronic wavefunction $\tilde{\Phi}_{e}(\mathbf{r})$, which need not be the true solution to the Schrödinger equation (2.4.4), the expectation value of the Hamiltonian:

$$
E\left[\tilde{\Phi}_{e}\right]=\int d \mathbf{r} \tilde{\Phi}_{e}^{*} \hat{H} \tilde{\Phi}_{e}
$$

corresponding to $\tilde{\Phi}_{e}$ will always be greater than or equal to the ground state energy $E_{0}$, i.e.:

$$
E\left[\tilde{\Phi}_{e}\right] \geq E_{0} \text { for all } \tilde{\Phi}_{e}
$$

The proof of this principle is simple. Since the Hamiltonian is a Hermitian operator ${ }^{157}$, all of its eigenfunctions are orthogonal:

$$
\int d \mathbf{r} \Phi_{\alpha}^{*} \Phi_{\beta}=\delta_{\alpha \beta}
$$


In (2.4.8) $\Phi_{\alpha}$ and $\Phi_{\beta}$ are two normalized eigenfunctions of the Hamiltonian and $\delta_{\alpha \beta}$ is the Kronecker delta. The eigenfunctions also form a complete basis for the space of all functions $\Phi(\mathbf{r})$, so any electronic wavefunction $\tilde{\Phi}_{e}(\mathbf{r})$ can be expanded in terms of the eigenfunctions as:

$$
\tilde{\Phi}_{e}(\mathbf{r})=\sum_{\alpha} c_{\alpha} \Phi_{\alpha}(\mathbf{r})
$$

where the $c_{\alpha}$ are constants. If we substitute (2.4.9) into (2.4.6) and use (2.4.8) together with the fact that the functions $\Phi_{\alpha}$ satisfy the Schrödinger equation (2.4.4), we obtain:

$$
E\left[\tilde{\Phi}_{e}\right]=\sum_{\alpha}\left|c_{\alpha}\right|^{2} E_{\alpha}
$$

Now, since the ground state energy is the smallest of the $E_{\alpha}$, this means that:

$$
E\left[\tilde{\Phi}_{e}\right]=\sum_{\alpha}\left|c_{\alpha}\right|^{2} E_{\alpha} \geq E_{0} \sum_{\alpha}\left|c_{\alpha}\right|^{2}=E_{0}
$$

The last equality follows from the fact that the $\Phi_{\alpha}$ are normalized. The variational principle provides a very good test of the quality of an approximation to the electronic wavefunction. Since the true ground state energy is a lower bound for the expectation energy, one always knows that, among different calculations with the same Hamiltonian, the one giving the lowest energy is the best. Note that this argument is good only if we are interested in the ground-state wavefunction. For an excited wavefunction (i.e. one corresponding to a higher energy), the true energy eigenvalue will be a lower bound only if the approximate wavefunction happens to be orthogonal to all the exact wavefunctions corresponding to the lower energies. Since in general we do not know these exact 
wavefunctions, the variational principle is not as useful in this case. Nevertheless, in many quantum chemistry applications we are interested only in finding the ground state wavefunction, and hence the variational principle remains very useful.

The variational principle actually provides a way to reformulate the problem of finding the ground state wavefunction. From (2.4.11) it is clear that the expectation value of the energy will be equal to $E_{0}$ only if $\tilde{\Phi}_{e}$ is the true ground state wavefunction (that is, if all the coefficients $c_{\alpha}$ for $\alpha \neq 0$ are zero). Hence finding the ground state wavefunction can be formulated as a variational minimization problem:

$$
\delta E\left[\Phi_{0}\right]=0
$$

This is the basis for many ab initio methods, some of which will be discussed in the following sections.

\subsubsection{Orbitals and Basis Functions ${ }^{158,159}$.}

The wavefunction appearing in the electronic problem (2.4.4) depends on the coordinates of all the electrons. For ease of both visualization and computation, it is convenient to express the electronic problem in terms of functions that depend only on the coordinates of a single electron. These functions are called orbitals. In the following sections we will see several ways to relate the many-electron wavefunction appearing in (2.4.4) to these orbitals. In this section we will discuss some common ways to represent these orbitals in $a b$ initio calculations. 
A spatial orbital $\psi_{i}(\mathbf{r})$ is a function of the position of a single electron $i$ defined such that the probability density of finding the electron in a position $\mathbf{r}$ is $\left|\psi_{i}(\mathbf{r})\right|^{2}$. The description of an electron also requires assigning a spin to it. When the spatial orbitals are combined with functions that specify the spin of the electron, $\sigma$, we obtain the spin orbitals $\chi_{i}(\mathbf{r}, \sigma)$. Since the spin of an electron can have two values ("spin up" and "spin down"), any given spatial orbital gives rise to two spin orbitals. Any single electron can be described by a single spin orbital.

In order to find approximate solutions to the electronic problem, it is necessary to assume a mathematical form for the spatial orbitals. This is usually done by expanding these orbitals in terms of a set of basis functions $\phi_{\mu}(\mathbf{r})$ :

$$
\psi_{i}(\mathbf{r})=\sum_{\mu=1}^{K} C_{\mu i} \phi_{\mu}(\mathbf{r})
$$

These basis functions are often, but not always, centered at the nuclei. When functions centered at different nuclei in a molecule are combined linearly as in (2.4.13), we have the LCAO-MO (Linear Combination of Atomic Orbitals Molecular Orbitals) representation. This is the most common way to represent molecular wavefunctions. Once a set of basis functions is chosen, the problem of finding the orbitals reduces to finding the expansion coefficients $C_{\mu i}$. In general, a larger basis set leads to a more accurate solution, but the computational cost typically increases as $O\left(K^{4}\right)$ or more, so it is imperative to choose basis functions that give good results with the lowest possible number of terms.

One set of basis functions that can be used for the spatial orbitals is inspired by the form of the analytical solution of the Schrödinger equation for the hydrogen atom. These functions 
are called Slater ${ }^{160}$ type orbitals (STOs). The normalized STO for a $1 \mathrm{~s}$ orbital centered at $\mathbf{R}_{A}$ is given by:

$$
\phi_{1 s}^{S T O}=\left(\frac{\zeta^{3}}{\pi}\right)^{1 / 2} \exp \left(-\zeta\left|\mathbf{r}-\mathbf{R}_{A}\right|\right)
$$

where $\zeta$, the Slater orbital exponent, is a parameter. In principle, this parameter should be adjusted together with the expansion coefficients $C_{\mu i}$ in (2.4.14). This however, makes the problem nonlinear and much more difficult to solve. There are published values of the best Slater exponents for each atomic species (see, for example, ref. 158), and these are normally used to avoid the nonlinearity. For orbitals other than the 1 , the STOs are multiplied by polynomials in the components of $\mathbf{r}-\mathbf{R}_{A}$.

STOs usually give very good results, but they have a major drawback. In most $a b$ initio methods, it is necessary to calculate integrals involving the orbitals in order to obtain the expectation values of all the terms in the Hamiltonian (2.4.3). These integrals do not have an analytical solution for STOs, and their numerical calculation adds a lot of computational cost. An alternative is to use Gaussian type orbitals ${ }^{161}$ (GTOs). For a $1 s$ orbital, the normalized GTO centered at $\mathbf{R}_{A}$ is:

$$
\phi_{1 s}^{G T O}=\left(\frac{2 \alpha}{\pi}\right)^{3 / 4} \exp \left(-\alpha\left|\mathbf{r}-\mathbf{R}_{A}\right|^{2}\right)
$$

where $\alpha$ is a parameter. It is possible to obtain analytical expressions for the expectation values of all terms in the Hamiltonian (2.4.3) when GTOs are used, hence reducing the computational cost considerably with respect to STO calculations. As with STOs, the 
orbitals other than $1 \mathrm{~s}$ are obtained by multiplying by polynomials in the components of $\mathbf{r}-\mathbf{R}_{A}$.

One major difference between STOs and GTOs is that the slope at $\mathbf{r}=\mathbf{R}_{A}$ of a GTO is always zero, which is not the case for the STOs. The GTOs also decay much faster with increasing distance. Since STOs usually give better results, one way to use GTOs but still keep some of the precision of the STOs is to fit the STOs using GTOs. The orbitals obtained in this way are called STO-NG orbitals, where the "NG" indicates the number of Gaussians used to fit the STO. For example, the STO-3G function is given by:

$$
\phi_{1 s}^{S T O-3 G}=d_{1} \phi_{1 s}^{G T O}\left(\alpha_{1}\right)+d_{2} \phi_{1 s}^{G T O}\left(\alpha_{2}\right)+d_{3} \phi_{1 s}^{G T O}\left(\alpha_{3}\right)
$$

where the $d_{i}$ are contraction coefficients. For a given STO, it is possible to find these coefficients and the corresponding Gaussian exponents $\alpha_{i}$ that provide the best fit. Using this kind of orbital considerably improves the computational speed with respect to an STO calculation, retaining much of its accuracy. Figure 2.4.1 shows how an increasingly better approximation is obtained by adding more GTOs to the expansion (2.4.16).

A way to incorporate more flexibility into the basis set is to allow for more than one Slater function to represent the same orbital. These orbitals are called multiple-zeta or split basis sets, and the most common are the double-zeta, which use two Slater functions for one orbital. Since in most cases the chemistry is determined by the valence electrons, it is common to use split basis functions only for them, and single STO-NGs for the core electrons. Such a basis set is called a split valence basis set. Split valence basis sets are usually labeled by three numbers (in the case of a double-zeta basis for the valence): one is the number of GTOs used to fit the STO for the core electrons, the other two are the numbers of GTOs used to fit the two STOs for the valence electrons. Hence, a 4-31G basis 
would consist of STO-4G functions for the core electrons, and a STO-3G and a STO-1G (the latter being effectively just one GTO) for the valence electrons. This can be extended to multiple-zeta basis for the valence, for example a $6-311 \mathrm{G}$ basis would use STO-6G functions for the core electrons, and one STO-3G and two GTOs for the valence electrons. Among the most common split-valence basis sets are the $3-21 \mathrm{G}^{162-164}, 4-31 \mathrm{G}^{165-167}$, 6$21 \mathrm{G}^{162-164}, 6-31 \mathrm{G}^{166,167}$ and $6-311 \mathrm{G}^{168-171}$.

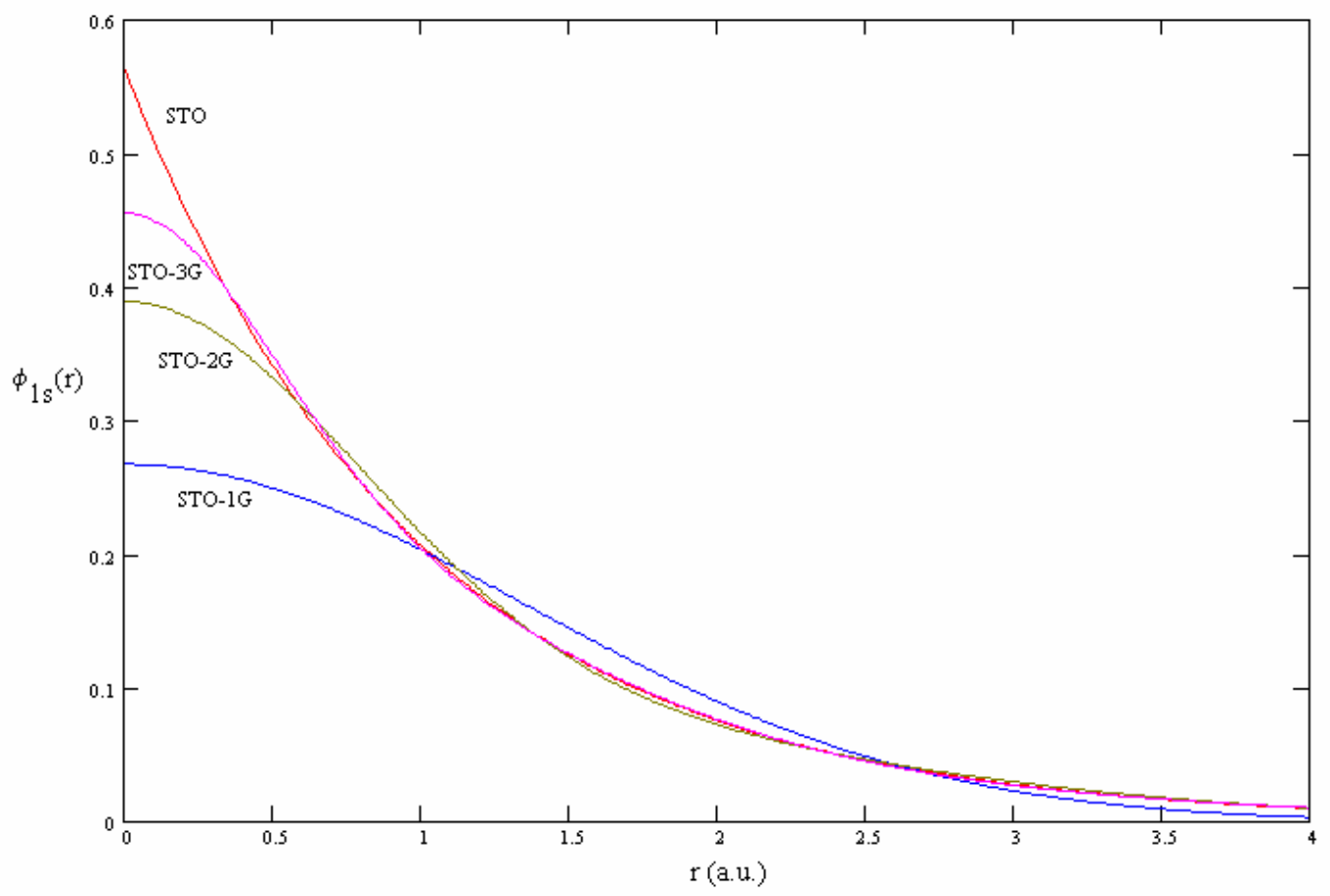

Figure 2.4.1. Three successive approximations to a hydrogen 1s Slater orbital using Gaussian functions.

Another improvement on the basis set is to include the possibility of polarization, that is contributions from higher angular momentum orbitals in the description. For example, a non-polarized basis set for a hydrogen atom would only contain s-type functions, but a polarized basis set would also include $p$-type or higher angular momentum functions. 
Polarized basis sets are usually labeled by adding one or more stars $(*)$ to their name, or by adding a list of the additional polarization functions used. For example, the $6-31 \mathrm{G}^{*}-$ or, equivalently, 6-31G $(d)$ - basis set ${ }^{172-175}$ for the first and second-row atoms in the periodic table is like the $6-31 \mathrm{G}$ but with an additional set of $3 d$ GTOs. The 6-31G** basis - or 6$31 \mathrm{G}(d, p)$ - also includes $p$-type GTOs for hydrogen. Including polarization gives more angular freedom so that the basis is able to represent bond angles more accurately, especially in strained ring molecules. Diffuse functions, which are additional GTOs with smaller exponents, can be also added for increased accuracy, and are denoted by adding "+" or "++" to the name. When "+" is used, it indicates the addition of a set of $s-$ and $p$ functions for the heavy atoms, whereas "++" indicates that an additional s- diffuse GTO for hydrogen. Diffuse functions are especially useful for cases where more radial flexibility is needed, such as the modeling of anions or Rydberg states ${ }^{176}$.

The basis sets discussed so far are among the most commonly used. With the increasing computational power available to quantum chemists, some more sophisticated basis sets have been developed. Among the most commonly used are the correlation-consistent basis sets of Dunning ${ }^{177-179}$, which include up to quintuple-zeta functions and polarization, and can be augmented with diffuse functions. These sets are usually labeled as (AUG-)ccpVNZ, where the prefix AUG- is added when diffuse functions are included, and $\mathrm{N}$ may be $\mathrm{D}$ (for double-zeta), T (triple-), Q (quadruple-) and 5 (quintuple-). A good description of these and other basis sets not discussed here can be found in ref. 5 , chapter 5 .

Another approach that is often used in solid-state calculations is to use plane wave basis sets (PWs), i.e. to represent the spatial orbitals as 3D Fourier expansions:

$$
\psi_{i}(\mathbf{r})=\sum_{\mu=1}^{K} C_{\mu i} e^{i \mathbf{k}_{\mu} \cdot \mathbf{r}}
$$


Using PW functions has several advantages, especially in the study of periodic solids and in $a b$ initio molecular dynamics (discussed in section 4.10). In the latter case, the basis functions that are centered on the nuclei give rise to contributions to the forces on the nuclei due to the fact that the basis functions' centers move in time. These contributions are sometimes called Pulay forces ${ }^{180}$, and are expensive to calculate ${ }^{181}$. Since PWs do not have a "center", there is no need to calculate these contributions in PW calculations. For a more detailed discussion of this point see ref. 181. Another advantage of PWs is that the calculation of forces can be done more easily in Fourier space. Since there are very efficient Fast Fourier Transform algorithms available, this decreases the computational cost of obtaining forces even further. PW basis sets are also very useful to study electrons that are not tightly bound to a nucleus, as in metals, and this is a reason for their popularity in solid-state calculations. In a sense, PW basis sets are complementary to atom-centered basis sets: a very good description of a material can be obtained by using atomic orbitals in the vicinity of the nuclei and plane waves in the internuclear regions. This is the basis for the muffin-tin approximation used in solid-state calculations. For an introduction to this treatment, see ref. 10. For a discussion of the relative advantages and disadvantages of PWs see ref. 181.

The main problem with PWs is that the wavefunctions tend to oscillate strongly in the core region due to the singularity of the Coulomb potential at $\mathbf{r}=\mathbf{0}$ and the requirement of orthogonality of the one-electron states. This adds very high-frequency components to the wavefunction, which require a large number of terms in the Fourier expansion (2.4.17). Since the valence shells are the most important for quantum chemistry, a way around this problem is to replace the core (i.e. nucleus + core electrons) by a potential that does not have a singularity at the origin. This potential is fitted to guarantee that the wavefunction of the valence electrons beyond a certain radius coincides with the "true" wavefunction (i.e. the one obtained when solving with the real potential and all the electrons). These potential functions are called atomic pseudopotentials (also known as effective core 
potentials), and their use permits obtaining good results with fewer terms in the expansion (2.4.17). The PW pseudopotential approach is very often used in solid-state calculations, and in $a b$ initio molecular dynamics (see section 2.4.10). Another advantage of the pseudopotential approach is that it is not necessary to solve for the core states, which reduces the computational cost significantly. Furthermore, in heavy atoms relativistic effects can be taken care of in the pseudopotential generation.

\subsubsection{Self-Consistent Mean-Field Theory - The Hartree-Fock Method}

The Hartree-Fock ${ }^{182-184}$ (HF) method is a reference for many other ab initio methods. Most of the modern picture of atomic and molecular structure in chemistry comes from the HF description. Since HF is the starting point for many other ab initio calculations, as well as the simpler semiempirical methods, we will discuss it first.

In the HF method, a many-electron system is treated using a collection of one-electron spin-orbitals. Each electron is assumed to belong to a different spin-orbital according to the

exclusion principle, and the electron-electron interactions are replaced by the interaction of each electron with the mean field resulting from the other electrons, as depicted in figure 2.4.2. Hence the HF method is a mean-field theory. This ansatz gives rise to an iterative procedure: each of the electronic wave functions will depend on the mean interaction with the other electrons, which in turn depends on the other electrons' wavefunctions. Therefore it is necessary to iterate with the set of wave functions until convergence is achieved. The resulting set of solutions after convergence is said to be self-consistent, and hence the name self-consistent mean-field theory. 


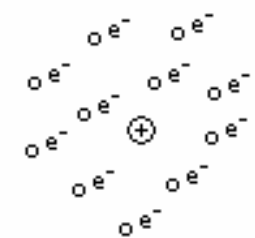

Many-body problem

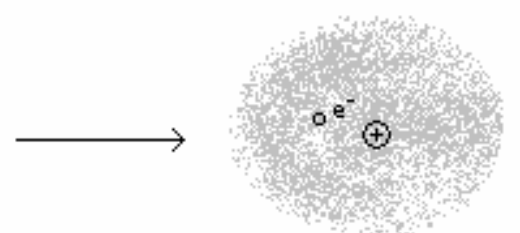

Approximate 2-body problem

Figure 2.4.2. The Hartree-Fock picture. The original many-body problem is transformed into a set of 2-body problems by replacing all but one of the electrons by a mean field. The equations for each electron are then solved self-consistently

In order to carry out this iterative procedure, we need to assume a form of the wave function for the many-electron system that contains some adjustable parameters. Since the many-electron wavefunction has to be antisymmetric, a possible mathematical form for it is the Slater ${ }^{185}$ determinant:

$$
\boldsymbol{\Psi}=\frac{1}{\sqrt{N !}}\left|\begin{array}{cccc}
\chi_{1}\left(\mathbf{r}_{1}\right) & \chi_{2}\left(\mathbf{r}_{1}\right) & \cdots & \chi_{N}\left(\mathbf{r}_{1}\right) \\
\chi_{1}\left(\mathbf{r}_{2}\right) & \chi_{2}\left(\mathbf{r}_{2}\right) & \cdots & \chi_{N}\left(\mathbf{r}_{2}\right) \\
\vdots & \vdots & \ddots & \vdots \\
\chi_{1}\left(\mathbf{r}_{N}\right) & \chi\left(\mathbf{r}_{N}\right) & \cdots & \chi_{N}\left(\mathbf{r}_{N}\right)
\end{array}\right|
$$

In (2.4.18), the $\chi_{i}$ are orthonormal spin-orbitals and the $1 / \sqrt{N !}$ is a normalization factor. Note that, since the exchange of two rows in a determinant changes its sign, this function satisfies the requirement of being antisymmetric upon exchange of two electrons. 
If the system under study is a closed-shell system, i.e. a system with equal numbers of electrons of either spin, (2.4.18) may be rewritten as:

$$
\boldsymbol{\Psi}=\frac{1}{\sqrt{N !}}\left|\begin{array}{cccc}
\psi_{1}\left(\mathbf{r}_{1}\right) \alpha\left(\sigma_{1}\right) & \psi_{2}\left(\mathbf{r}_{1}\right) \beta\left(\sigma_{1}\right) & \cdots & \psi_{N}\left(\mathbf{r}_{1}\right) \beta\left(\sigma_{1}\right) \\
\psi_{1}\left(\mathbf{r}_{2}\right) \alpha\left(\sigma_{2}\right) & \psi_{2}\left(\mathbf{r}_{2}\right) \beta\left(\sigma_{2}\right) & \cdots & \psi_{N}\left(\mathbf{r}_{2}\right) \beta\left(\sigma_{2}\right) \\
\vdots & \vdots & \ddots & \vdots \\
\psi_{1}\left(\mathbf{r}_{2 N}\right) \alpha\left(\sigma_{2 N}\right) & \psi_{2}\left(\mathbf{r}_{2 N}\right) \beta\left(\sigma_{2 N}\right) & \cdots & \psi_{N}\left(\mathbf{r}_{2 N}\right) \beta\left(\sigma_{2 N}\right)
\end{array}\right|
$$

where the $\psi_{i}$ are orthonormal spatial orbitals and $\alpha, \beta$ are functions determining the spin of the electron. In this case we have a restricted Hartree-Fock (RHF) calculation ${ }^{186}$. When the system under study is not a closed-shell system, it is necessary to perform an unrestricted Hartree-Fock calculation (UHF), which treats the electrons with different spins differently. Since the main ideas behind the method are the same in both RHF and UHF, we will describe the simplest one (RHF). For a detailed treatment of UHF see, for example, refs. 5 and 7.

Using (2.4.19), the expectation value of the Hamiltonian can be written as:

$$
E[\Psi]=2 \sum_{i=1}^{N} H_{i i}-\sum_{i=1}^{N} \sum_{j=1}^{N}\left(2 J_{i j}-K_{i j}\right)
$$

where the $H_{i i}$ contain the contributions of the kinetic energy and the potential energy of the electron-nuclei interactions:

$$
H_{i i}=\int d \mathbf{r} \psi_{i}^{*}\left[-\frac{1}{2} \nabla^{2}-\sum_{I} \frac{Z_{I}}{r_{i I}}\right] \psi_{i}
$$


The index $I$ in (2.4.21) runs over all the nuclei in the molecule. The terms $J_{i j}$ in $(2.4 .20)$ are called the Coulomb integrals and represent the potential energy of the interactions of electrons with orbitals $\psi_{i}$ and $\psi_{j}$ :

$$
J_{i j}=\iint d \mathbf{r}_{1} d \mathbf{r}_{2} \psi_{i}^{*}\left(\mathbf{r}_{1}\right) \psi_{j}^{*}\left(\mathbf{r}_{2}\right) \frac{1}{r_{12}} \psi_{i}\left(\mathbf{r}_{1}\right) \psi_{j}\left(\mathbf{r}_{2}\right)
$$

Finally, the terms $K_{i j}$ in (2.4.20) are the exchange integrals, which arise from the requirement that the wavefunction be antisymmetric, and represent the "exclusion principle" contribution to the energy:

$$
K_{i j}=\iint d \mathbf{r}_{1} d \mathbf{r}_{2} \psi_{i}^{*}\left(\mathbf{r}_{1}\right) \psi_{j}^{*}\left(\mathbf{r}_{2}\right) \frac{1}{r_{12}} \psi_{i}\left(\mathbf{r}_{2}\right) \psi_{j}\left(\mathbf{r}_{1}\right)
$$

Now that we have an expression for the energy, we can apply the variational principle. The value that minimizes $E$ in (2.4.20), subject to the restriction that the orbitals be orthonormal, is the solution to our problem. Applying the method of Lagrange multipliers to the minimization problem we obtain:

$$
\left[\hat{H}_{n}+\sum_{j=1}^{N}\left(2 \hat{J}_{j}-\hat{K}_{j}\right)\right] \psi_{i}=\hat{F} \psi_{i}=\varepsilon_{i} \psi_{i}
$$

where we defined the Fock operator $\hat{F}$. It is defined in terms of the nuclei-electron Hamiltonian $\hat{H}_{n}$ :

$$
\hat{H}_{n} \psi_{i}\left(\mathbf{r}_{1}\right)=-\frac{1}{2} \nabla^{2}-\sum_{I} \frac{Z_{I}}{r_{i I}}
$$


the Coulomb operators $\hat{J}_{j}$ :

$$
\hat{J}_{j} \psi_{i}\left(\mathbf{r}_{1}\right)=\int d \mathbf{r}_{2} \psi_{j}^{*}\left(\mathbf{r}_{2}\right) \frac{1}{r_{12}} \psi_{j}\left(\mathbf{r}_{2}\right) \psi_{i}\left(\mathbf{r}_{1}\right)
$$

and the exchange operators $\hat{K}_{j}$ :

$$
\hat{K}_{j} \psi_{i}\left(\mathbf{r}_{1}\right)=\int d \mathbf{r}_{2} \psi_{j}^{*}\left(\mathbf{r}_{2}\right) \frac{1}{r_{12}} \psi_{i}\left(\mathbf{r}_{2}\right) \psi_{j}\left(\mathbf{r}_{1}\right)
$$

The $\varepsilon_{i}$ in equation (2.4.24) are the Lagrange multipliers, called the orbital energies, and include the kinetic energy and the energy of the nuclei-electron interactions for each orbital. In order to minimize (2.4.24) we need to choose a form for the orbitals, i.e. expand them in terms of some basis functions as discussed in section 4.3. By substituting the expansion (2.4.13) into (2.4.24), multiplying both sides by $\phi_{\mu}$ and integrating over all space, we obtain the Roothaan equations ${ }^{187,188}$ (also known as the secular equations, or Roothaan-Hall ${ }^{189}$ equations):

$$
\sum_{v=1}^{K} C_{v i}\left(F_{\mu v}-\varepsilon_{i} S_{\mu v}\right)=0
$$

where:

$$
F_{\mu v}=\int d \mathbf{r} \phi_{\mu}^{*} \hat{F} \phi_{v}
$$


are the matrix elements of the Fock operator in the given basis, and:

$$
S_{\mu v}=\int d \mathbf{r} \phi_{\mu}^{*} \phi_{v}
$$

are the elements of the overlap matrix. There is one secular equation for each value of $i, \mu$ from 1 to $K$. For the solution to be nontrivial, the determinant of this system of equations (the secular determinant) has to be zero. Solving this determinant equation for each orbital $i$ gives the values of the orbital energies $\varepsilon_{i}$, and then the secular equations (2.4.28) can be solved to obtain the coefficients (except for one, which can be given an arbitrary value).

The general procedure for obtaining a HF wave function is hence as follows:

(1) Choose a set of basis functions $\phi_{\mu}$ and guess initial values for the coefficients $C_{\mu i}$ for each orbital. Calculate the elements $S_{\mu v}$ of the overlap matrix.

(2) Calculate the matrix elements of the Fock operator $F_{\mu v}$, find the orbital energies $\varepsilon_{i}$ by setting the secular determinants to zero, and solve (2.4.28) in order to obtain improved values of the coefficients $C_{\mu i}$.

(3) With the new $C_{\mu i}$, return to step (2). Repeat until convergence is achieved.

(4) Once the $C_{\mu i}$ are known, equations (2.4.19) and (2.4.20) can be used to construct the approximate all-electron wave function and the energy. 
The most computationally expensive part of the HF procedure is to evaluate the integrals needed to build the Fock matrix defined by (2.4.29). The reason for this can be seen by substituting the definition of the Fock operator into the expression for its matrix element:

$$
\begin{aligned}
& F_{\mu v}=\int d \mathbf{r} \phi_{\mu}^{*}\left[\begin{array}{l}
\left.-\frac{1}{2} \nabla^{2}-\sum_{I} \frac{Z_{I}}{r_{i I}}\right] \phi_{v}+ \\
\sum_{j=1}^{N / 2} \sum_{\lambda=1}^{K} \sum_{\sigma=1}^{K} C_{\lambda j} C_{\sigma j}\left[\begin{array}{l}
2 \iint d \mathbf{r}_{1} d \mathbf{r}_{2} \phi_{\mu}^{*}\left(\mathbf{r}_{1}\right) \phi_{v}^{*}\left(\mathbf{r}_{1}\right) \frac{1}{r_{12}} \phi_{\lambda}\left(\mathbf{r}_{2}\right) \phi_{\sigma}\left(\mathbf{r}_{2}\right)- \\
\iint d \mathbf{r}_{1} d \mathbf{r}_{2} \phi_{\mu}^{*}\left(\mathbf{r}_{1}\right) \phi_{\lambda}^{*}\left(\mathbf{r}_{1}\right) \frac{1}{r_{12}} \phi_{v}\left(\mathbf{r}_{2}\right) \phi_{\sigma}\left(\mathbf{r}_{2}\right)
\end{array}\right]= \\
=\int d \mathbf{r} \phi_{\mu}^{*}\left[-\frac{1}{2} \nabla^{2}-\sum_{I} \frac{Z_{I}}{r_{i I}}\right] \phi_{v}+\sum_{j=1}^{N / 2} \sum_{\lambda=1}^{K} \sum_{\sigma=1}^{K} C_{\lambda j} C_{\sigma j}[2(\mu v \mid \lambda \sigma)-(\mu \lambda \mid v \sigma)]
\end{array}\right.
\end{aligned}
$$

where we have introduced the shorthand notation $^{190}(\mu \nu \mid \lambda \sigma)$ for the two-electron integrals:

$$
(\mu \nu \mid \lambda \sigma) \equiv \iint d \mathbf{r}_{1} d \mathbf{r}_{2} \phi_{\mu}^{*}\left(\mathbf{r}_{1}\right) \phi_{v}^{*}\left(\mathbf{r}_{1}\right) \frac{1}{r_{12}} \phi_{\lambda}\left(\mathbf{r}_{2}\right) \phi_{\sigma}\left(\mathbf{r}_{2}\right)
$$

Note that the number of two-electron integrals needed for a basis set containing $K$ functions grows as $O\left(K^{4}\right)$. This is the reason why it is important to use basis functions that can be integrated analytically, as mentioned in section 2.4.3.

One important advantage of the HF method is that it provides a basis to interpret the properties of atoms and molecules in terms of simple orbitals. The HF representation is the basis for most of the modern picture of the electronic structure of atoms. The HF method also has a low computational cost, as compared to most other $a b$ initio methods, which makes it able to treat larger systems. Actually, in many cases HF is used to obtain a first 
approximation to the solution (and a basis for the space of many-electron wavefunctions, as explained in the next section).

The main disadvantage of $\mathrm{HF}$ is that the potential energy of the electron-electron interaction is treated as an average, i.e. each electron is assumed to interact with the average force field produced by the other electrons. This neglects the contribution from the instantaneous electron-electron potential energy, which does not average to zero. This energy is called the correlation energy and it is an important contribution ${ }^{191}$. Many other $a b$ initio methods attempt to correct this by using HF to generate a first approximation and then add terms to account for the correlation energy.

As opposed to force-field methods, in the HF method it is possible to systematically improve on the results by using larger basis sets. In the limit of an infinitely large basis set, the HF limit, the only error is the correlation energy. After that point, the only possible improvement is to use a different method that is able to estimate the correlation energy.

Starting with the HF method, there are different pathways that lead to different methods, as depicted in figure 2.4.3. One option is to develop higher-level methods to calculate the correlation energy and thus improve the HF results. This gives rise to the families of correlation energy methods discussed in the next three sections. The problem with these approaches, as we will see, is that they increase the computational cost and hence are only applicable to relatively small systems. The other option is to add simplifications to the HF method, and include adjustable parameters that are fitted so that the calculations reproduce experimental data. This gives rise to the family of semi-empirical methods, which have a much lower computational cost and hence can be applied to larger systems. Although these methods are not truly $a b$ initio methods, because they rely on experimental data, they also play an important role in the study of chemical reactions and so we will discuss them briefly in section 2.4.8. 


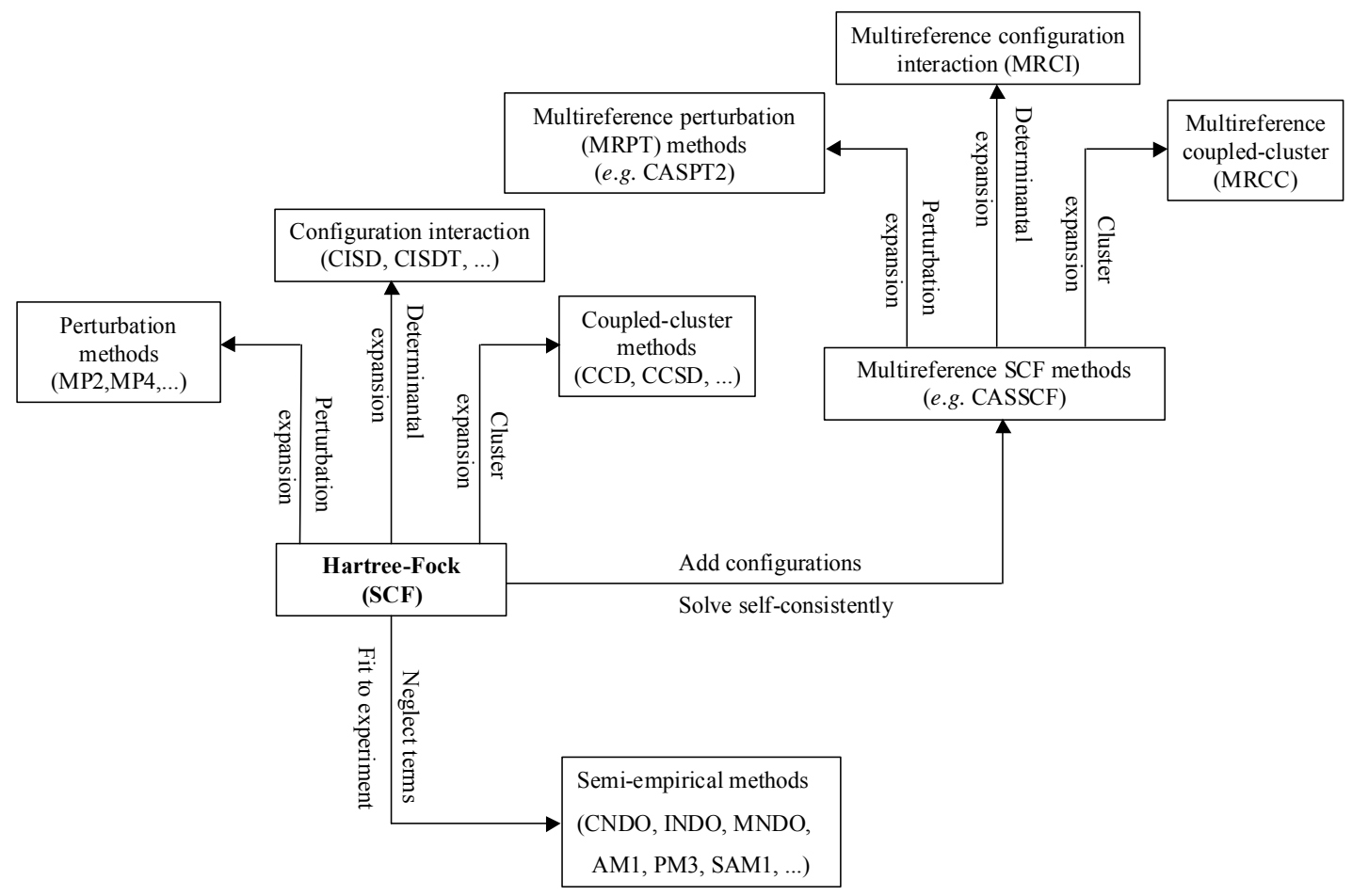

Figure 2.4.3. Connection between many of the $a b$ initio methods. The terms in this schematic are explained in the following sections.

Applications of the HF method, by itself, are nowadays more often in the study of complex systems, such as large molecules, surfaces and solutions. HF calculations are, however, used as a starting point for many other higher-level methods. Some recent studies involving purely HF calculations, showing the kind of systems that can be handled by it with the current computational power, are:

- A study of the charge distribution in spodumene ${ }^{192}$, showing the different electron distributions around the nonequivalent oxygen atoms in the mineral's structure. The structural features of spodumene obtained in this work showed a reasonably good agreement with experimental results from x-ray and neutron diffraction studies. 
- A conformational analysis of the hormone melatonin ${ }^{193}$, which considered all the torsional angles relevant for the description of the conformational PES of the molecule.

- A recent benchmark calculation of the interaction energy of streptavidin and biotin ${ }^{194}$, using a system of 1775 atoms. The calculations in this work were made using a new linear-scaling computational method. Several methods that are linear- or almost linearscaling are currently in development that can make $a b$ initio calculations affordable for complex systems in the near future. For examples of these techniques, see refs. 195, 196.

\subsubsection{Configuration Interaction and Other Variational Methods.}

There is an important observation to make about the HF solutions. Since there are $K$ equations in (2.4.28), there will be as many spatial orbitals as there are basis functions. If the number of basis functions $K$ were equal to $N$, i.e. half the number of electrons, there would be only one way to build the Slater determinant (2.4.18) and one set of orbital energies. However, if the basis set is larger (which is usually the case), there are more than $N$ spatial orbitals $\psi_{i}$ and energies $\varepsilon_{i}$ in the solution. The ground state of the system will be the Slater determinant corresponding to the $N$ lowest-energy eigenvalues. The next higher energy eigenvalues are approximations to the energies of the excited states of the molecule. There is in fact a result, known as Koopmans' theorem ${ }^{197}$, which relates the energies of the virtual (i.e. unoccupied) orbitals to the electron affinities ${ }^{198}$. One may also build additional excited determinants by replacing one or more orbitals in the Slater determinant (2.4.18) with orbitals corresponding to higher energies. Thus one may build singly-excited, doublyexcited, and so on determinants. These determinants are usually not good approximations for the real excited many-electron wavefunctions of the system, because electronic excitations are often collective effects and neglecting the correlation energy leads to major errors. Excited determinants, however, are important for another reason. Since they are the 
eigenfunctions of the many-electron Hartree-Fock Hamiltonian, they form a basis for the space of antisymmetric many-electron wavefunctions. Hence the exact many-electron wavefunction may be written as a linear combination of the excited determinants as:

$$
\Phi_{e}=c_{0} \boldsymbol{\Psi}_{0}+\sum_{a, r} c_{a}^{r} \boldsymbol{\Psi}_{a}^{r}+\sum_{\substack { a, r \\
\begin{subarray}{c}{b>a \\
s>r{ a , r \\
\begin{subarray} { c } { b > a \\
s > r } }\end{subarray}} c_{a b}^{r s} \Psi_{a b}^{r s}+\cdots
$$

In (2.4.33), $\boldsymbol{\Psi}_{0}$ is the Hartree-Fock many-electron wavefunction. The symbol $\boldsymbol{\Psi}_{a}^{r}$ indicates a singly excited determinant ${ }^{186}$ in which the $a$-th spin orbital has been replaced by the $r$-th excited spin orbital. The symbol $\Psi_{a b}^{r s}$ means that also the $b$-th spin orbital has been replaced by the $s$-th excited spin orbital, and so on. These contributions are depicted in figure 2.4.4. The second summation is made over $b>a$ and $s>r$ to avoid counting the same doubly excited determinant twice. The expansion (2.4.33) would be infinite if the basis set were infinite. For the case of a basis set with $K$ functions, there are $\left(\begin{array}{c}2 K \\ N\end{array}\right)$ excited determinants, and the basis set is not complete. Nevertheless, if all the excited determinants are included in the expansion, the expression (2.4.33) gives an exact representation of the many-electron wavefunction within the function space spanned by the chosen orbital basis. The expansion (2.4.33) is the basis for the configuration interaction (CI) methods.

The basic idea of CI is to find the coefficients in the expansion (2.4.33) by applying the variational principle (2.4.12) to the all-electron wavefunction $\Phi_{e}$. Actually, if the basis set used for the electronic orbitals were infinite, finding all the coefficients appearing in (2.4.33) would lead to the exact solution to the electronic Schrödinger equation (2.4.4). Of course, we cannot work with an infinite basis set, so the next step would be using a set with a finite number $K$ of basis functions. If all the terms in the (now finite but large) expansion (2.4.33) are used, the CI result would be the exact solution to the electronic 
Schrödinger equation within the function space spanned by the chosen basis. This is called a full CI calculation. The problem with full CI is that its computational cost grows more than exponentially with the number of electrons, making it impractical for all but the smallest systems. Yet full $\mathrm{CI}$ is often used as a reference to benchmark the accuracy of other methods. One of the benchmark requirements usually imposed to modern $a b$ initio methods is to be equivalent to full CI when applied to a two-electron system ${ }^{199,200}$.

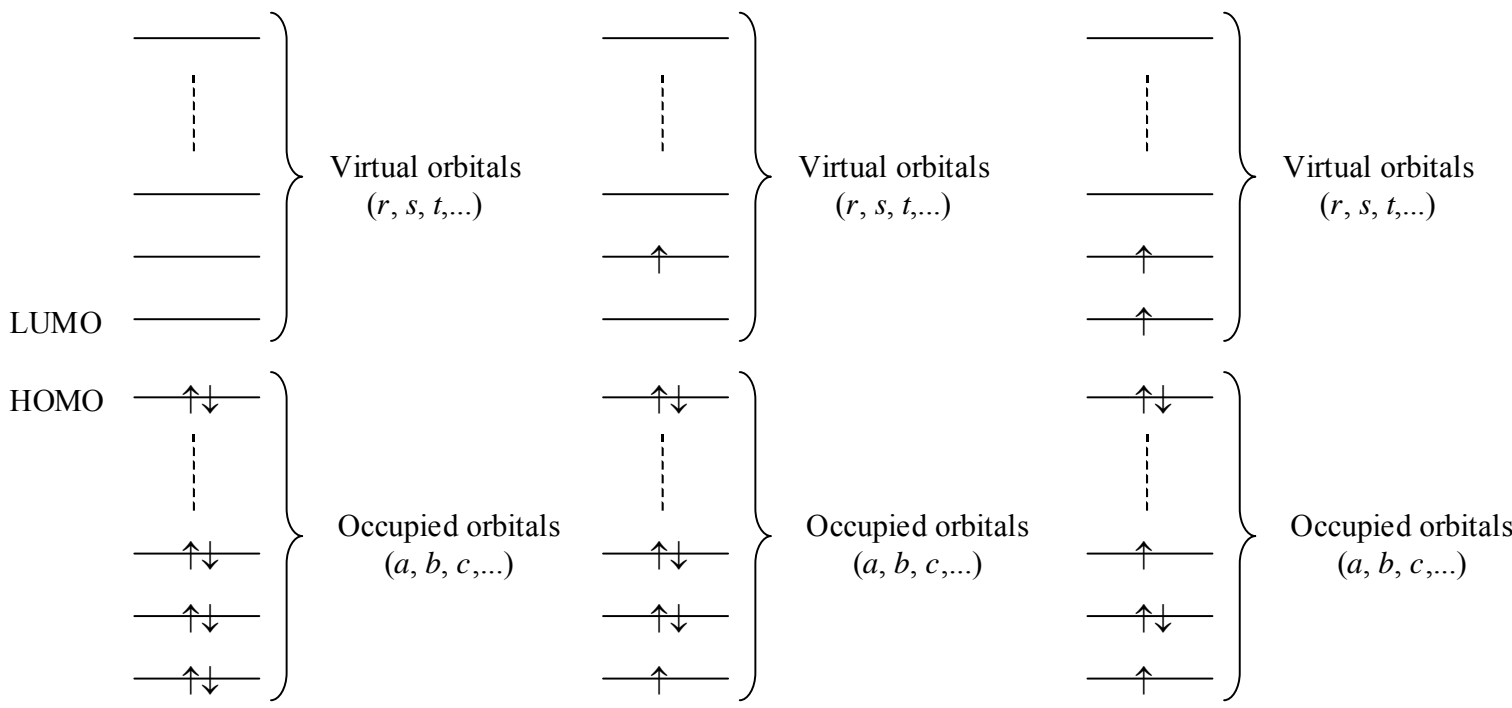

Figure 2.4.4. Excited configurations. HOMO and LUMO stand for "highest occupied molecular orbital" and "lowest unoccupied molecular orbital".

For systems large enough to be impractical for a full CI calculation, it is still possible to do a CI calculation by considering only some of the terms in the expansion (2.4.33). This gives rise to the CIS (CI with single excitations), CID (CI with double excitations), CISD (CI with single and double excitations), CISDT (CISD plus triple excitations), CISDQ (CISD with quadruple excitations) and CISDTQ (CISD plus triple and quadruple 
excitations) methods. Very seldom are higher order excitations taken into account because of the computational cost involved.

A CI calculation where the coefficients of the orbitals in (2.4.13) are also optimized is an example of a Multiconfigurational Self-Consistent Field (MCSCF) method ${ }^{5}$. Besides truncating the full CI expansion, it is possible to select the configurations that make up the all-electron wavefunction in a different way. One broadly used option is the Complete Active Space Self-Consistent Field method ${ }^{201}$ (CASSCF). In this method, the molecular orbitals are divided into inactive orbitals, which are always doubly occupied, and active orbitals. The CASSCF method consists of including all excitations for only the active orbitals, which usually correspond to the valence states ${ }^{202}$. Since the chemistry is determined principally by them, this is usually a very good approximation, and has a much lower computational cost than a full CI calculation, especially for heavy atoms. Another method on the lines of MCSCF is the Generalized Valence Bond (GVB) method ${ }^{203,204}$, in which two different configurations are used to form the all electron wavefunction. The GBV method is an extension of the Valence Bond (VB) method, originally introduced by Heitler and London to study the hydrogen molecule in $1927^{205,206}$. The VB method is an alternative to the molecular orbital description more commonly used in ab initio methods. Recent accounts of VB methods including modern VB theory are in refs. 207-211. It is also possible to use multireference all-electron wavefunctions in a determinantal expansion like (2.4.13) to obtain a multireference CI (MRCI) method

An advantage that is common to all variational methods is the very fact that they use the variational principle. Hence, it is easy to compare two different results: the one giving the lowest ground-state energy is the most accurate. On the other hand, many of these methods have what is called the size-extensivity problem (exceptions are, for example, full $\mathrm{CI}$ and CASSCF if the active space is chosen appropriately). Let us assume that we want to describe the bond between two atoms $\mathrm{A}$ and $\mathrm{B}$ to form a molecule $\mathrm{AB}$. If we do a CISD 
calculation to find the energies of both $\mathrm{A}, \mathrm{B}$ and $\mathrm{AB}$, the results are not consistent. The reason is that a double excitation for $\mathrm{A}$ and $\mathrm{B}$ atoms would be a quadruple excitation for $\mathrm{AB}$. The result of this is that a CISD calculation for a system containing an $\mathrm{A}$ and a $\mathrm{B}$ atom very far from each other would give a different energy than the sum of energies from separate calculations with A and B, which is physically absurd. One way around this problem is to use a perturbation method or a coupled-cluster method; these are described in the next sections.

Unlike the HF method, in the CI methodology there are two different ways to improve on the results: one is to use a larger basis set, and the other is to include more excitations in the CI expansion. Also unlike HF, the CI method can in principle give results arbitrarily close to the exact solution, by letting the basis set and the CI expansion both become large.

Typical applications of CI methods to chemical reactions are usually restricted to simple systems due to their computational cost. Most recent applications employ the more sophisticated CI methods, like CASSCF and multireference CI. Some recent representative applications of CI and other variational methods are:

- A study of the concerted and stepwise mechanisms of the hetero-Diels-Alder reaction of butadiene with formaldehyde $\left(\mathrm{C}_{4} \mathrm{H}_{6}+\mathrm{HCHO} \rightarrow \mathrm{C}_{5} \mathrm{H}_{8} \mathrm{O}\right)$ and thioformaldehyde $\left(\mathrm{C}_{4} \mathrm{H}_{6}+\mathrm{HCHS} \rightarrow \mathrm{C}_{5} \mathrm{H}_{8} \mathrm{~S}\right)$ using the CASSCF method ${ }^{212}$. The Diels-Alder reactions have a notoriously complex reaction mechanism, and have been the subject of many $a b$ initio studies.

- A study of the PES for the charge exchange between different cations $\left(\mathrm{H}^{+}, \mathrm{Na}^{+}, \mathrm{C}^{+}\right.$and $\mathrm{S}^{+}$) and a lithium fluoride surface using several CI approaches and also a coupledcluster method $^{213}$.

- An analysis of the electronic spectrum of the SiSe molecule ${ }^{214}$, giving a good agreement with experimentally observed electronic transitions. 


\subsubsection{Perturbation Methods.}

Once an approximate solution to the electronic problem (2.4.4) is known, e.g. from a HF calculation, it is possible to obtain an improved wave function through perturbation theory. In the Rayleigh-Schrödinger (RS) approach, we start by assuming that we have a first approximate set of many-electron wavefunctions $\Psi_{i}^{(0)}$, which are eigenfunctions of an approximate Hamiltonian $\hat{H}_{0}$ :

$$
\hat{H}_{0} \Psi_{i}^{(0)}=E_{i}^{(0)} \Psi_{i}^{(0)}
$$

We can now define a perturbation operator $\hat{V}$ as the difference between the exact electronic Hamiltonian $\hat{H}_{e}$ and the approximate Hamiltonian $\hat{H}_{0}$. As is usually done in perturbation theory, we will introduce a perturbation parameter $\lambda$, which varies between 0 and 1 , and define the functions:

$$
\begin{aligned}
& \Psi_{\lambda, i}(\lambda)=\Psi_{i}^{(0)}+\lambda\left(\Phi_{i}-\Psi_{i}^{(0)}\right) \\
& E_{\lambda, i}(\lambda)=E_{i}^{(0)}+\lambda\left(E_{i}-E_{i}^{(0)}\right)
\end{aligned}
$$

where $\Phi_{i}$ and $E_{i}$ are the eigenfunctions and eigenvalues of $\hat{H}_{e}$. It is clear that these functions give the approximate $\Psi_{i}^{(0)}$ and $E_{i}^{(0)}$ for $\lambda=0$, and approach the exact solutions as $\lambda \rightarrow 1$. If we expand these functions in a Taylor series in $\lambda$ we get:

$$
\begin{gathered}
\Psi_{\lambda, i}=\Psi_{i}^{(0)}+\lambda \Psi_{i}^{(1)}+\lambda^{2} \Psi_{i}^{(2)}+O\left(\lambda^{3}\right) \\
E_{\lambda, i}=E_{i}^{(0)}+\lambda E_{i}^{(1)}+\lambda^{2} E_{i}^{(2)}+O\left(\lambda^{3}\right)
\end{gathered}
$$


It is possible to take more terms in the series into account; we will just show the procedure for second-order perturbation here. In (2.4.36) and (2.4.37) we have defined:

$$
\begin{aligned}
\Psi_{i}^{(1)} \equiv\left(\frac{\partial \Psi_{\lambda, i}}{\partial \lambda}\right)_{\lambda=0} & \Psi_{i}^{(2)}=\frac{1}{2}\left(\frac{\partial^{2} \Psi_{\lambda, i}}{\partial \lambda^{2}}\right)_{\lambda=0} \\
E_{i}^{(1)}=\left(\frac{\partial E_{\lambda, i}}{\partial \lambda}\right)_{\lambda=0} & E_{i}^{(2)}=\frac{1}{2}\left(\frac{\partial^{2} E_{\lambda, i}}{\partial \lambda^{2}}\right)_{\lambda=0}
\end{aligned}
$$

From the definition of the perturbation operator $\hat{V}$, we have:

$$
\hat{H}_{e}=\hat{H}_{0}+\hat{V}
$$

Substituting equations (2.4.36), (2.4.37) and (2.4.40) into the electronic Schrödinger equation (2.4.4) we get:

$$
\begin{aligned}
& \left(\hat{H}_{0}+\hat{V}\right)\left[\Psi_{i}^{(0)}+\lambda \Psi_{i}^{(1)}+\lambda^{2} \Psi_{i}^{(2)}+O\left(\lambda^{3}\right)\right]= \\
& =\left[E_{i}^{(0)}+\lambda E_{i}^{(1)}+\lambda^{2} E_{i}^{(2)}+O\left(\lambda^{3}\right)\right]\left[\Psi_{i}^{(0)}+\lambda \Psi_{i}^{(1)}+\lambda^{2} \Psi_{i}^{(2)}+O\left(\lambda^{3}\right)\right]
\end{aligned}
$$

which can be rewritten as:

$$
\begin{aligned}
& \left(\hat{H}_{0}-E_{i}^{(0)}\right) \Psi_{i}^{(0)}+\lambda\left[\hat{H}_{0} \Psi_{i}^{(1)}+\hat{V} \Psi_{i}^{(0)}-E_{i}^{(0)} \Psi_{i}^{(1)}-E_{i}^{(1)} \Psi_{i}^{(0)}\right]+ \\
& +\lambda^{2}\left[\hat{H}_{0} \Psi_{i}^{(2)}+\hat{V} \Psi_{i}^{(1)}-E_{i}^{(0)} \Psi_{i}^{(2)}-E_{i}^{(1)} \Psi_{i}^{(1)}-E_{i}^{(2)} \Psi_{i}^{(0)}\right]+O\left(\lambda^{3}\right)=0
\end{aligned}
$$


Now, equating like powers of $\lambda$ on both sides we get:

$$
\left(\hat{H}_{0}-E_{i}^{(0)}\right) \Psi_{i}^{(0)}=0
$$

and:

$$
\begin{gathered}
\hat{H}_{0} \Psi_{i}^{(1)}+\hat{V} \Psi_{i}^{(0)}-E_{i}^{(0)} \Psi_{i}^{(1)}-E_{i}^{(1)} \Psi_{i}^{(0)}=0 \\
\hat{H}_{0} \Psi_{i}^{(2)}+\hat{V} \Psi_{i}^{(1)}-E_{i}^{(0)} \Psi_{i}^{(2)}-E_{i}^{(1)} \Psi_{i}^{(1)}-E_{i}^{(2)} \Psi_{i}^{(0)}=0
\end{gathered}
$$

Equation (2.4.43) tells us something we already knew: that $\Psi_{i}^{(0)}$ and $E_{i}^{(0)}$ are the eigenfunctions and eigenvalues of $\hat{H}_{0}$. Equation (2.4.44) gives the first-order corrections and (2.4.45) gives the second-order corrections. If more terms are used in the expansions (2.4.36)-(2.4.37), higher order corrections can be obtained. In order to solve equation (2.4.44), we express the first-order perturbation function $\Psi_{i}^{(1)}$ as a combination of the $\Psi_{i}^{(0)}$ orthonormal functions:

$$
\Psi_{i}^{(1)}=\sum_{k} C_{i k} \Psi_{k}^{(0)}
$$

For example, if $\hat{H}_{0}$ is taken to be the many-electron Hartree-Fock Hamiltonian, this expansion would be equivalent to the expansion (2.4.33). Substitution of (2.4.46) into (2.4.44) yields:

$$
\left(\hat{H}_{0}-E_{i}^{(0)}\right) \sum_{k} C_{i k} \Psi_{k}^{(0)}=-\left(\hat{V}-E_{i}^{(1)}\right) \Psi_{i}^{(0)}
$$


Taking the inner product on both sides with $\Psi_{j}^{(0)}$ and using $(2.4 .43)$ and the orthonormality conditions (2.4.8) we get:

$$
\left(E_{j}^{(0)}-E_{i}^{(0)}\right) C_{i j}=E_{i}^{(1)} \delta_{i j}-\int d \mathbf{r} \Psi_{j}^{(0) *} \hat{V} \Psi_{i}^{(0)}
$$

This set of equations allows us to obtain the coefficients in the expansion (2.4.46) and the energies $E_{i}^{(1)}$. A similar procedure can be used to obtain the second-order corrections, starting with equation (2.4.45).

What we have described above is the general RS perturbation theory. A more detailed treatment of it can be found, for example, in ref. 7. Evidently the details of the solution will depend on the choice of the approximate Hamiltonian $\hat{H}_{0}$. By far the most common choice is to use the Hartree-Fock Hamiltonian as the reference; in this case we obtain the MøllerPlesset $^{215}$ (MP) perturbation theory ${ }^{216-221}$. Usually the MP methods are referred to as MPN, where $N$ is the order of the corrections used. There is really no such thing as MP1, because the first correction to the Hartree-Fock energies actually arises from the second-order term ${ }^{7}$. The most common implementations are MP2 $2^{218-220}$ and MP4 $4^{221}$, but other levels of approximation are available.

It is possible to develop a perturbation theory using different references. In the complete active space perturbation theory (CASPT) method $^{222}$, for example, the reference is obtained from a CASSCF calculation. CASPT is an example of a multi-reference perturbation method, where a MCSCF method is used for the zero-th order approximation.

A major advantage of perturbation approaches is that they do not suffer from sizeextensivity problems. They can also handle excited states more easily than most variational methods. Nevertheless, the fact that they are not variational means that one cannot directly 
assess the relative quality of two different perturbation results. Since different-order perturbations correspond effectively to different Hamiltonians, one cannot invoke the variational principle and say that the lowest ground-state energy is the most accurate one. As is the case with the lower-level CI methods, the simpler perturbation approaches are being displaced by DFT for some applications.

As for the CI methods, perturbation results can be systematically improved in two different ways: by using larger basis sets or by including higher-order corrections. In principle, in the limit of an infinitely large basis set and an infinite expansion, the result should be correct. This, however, is true only if the perturbation expansion converges: if the reference state is too far removed from the actual state, the perturbation may diverge. Unlike CI, in perturbation theory the number of terms in the expansion and the number of basis functions are truly independent.

Some recent applications where perturbation methods are applied, showing typical systems that can be studied with them, are:

- A study of the reaction mechanism of the gas-phase hydrolysis of silicon tetrachloride ${ }^{223}$ using MP2, MP4 and DFT methods. In this study, good agreement with experimental reaction rates was obtained at high temperatures, but not for low temperatures.

- A study of the PES for the tautomeric and conformational rearrangements of 2nitrosophenol and 9,10-phenanthrenequinonemonooxime, using both the MP2 and MP4 methods ${ }^{224}$. The results obtained for both compounds were found to be in good agreement with experimental measurements, including NMR spectroscopy results obtained in the same work.

- A study of the reaction mechanism of the diazetization reactions of several azoalkanes, including one-, two- and three-bond mechanisms ${ }^{225}$. In this work, the CASPT2 method 
was used to calculate the energetics for this reaction, in conjunction with geometry optimizations using DFT and CASSCF. Figure 2.4.5 shows one of the mechanisms obtained

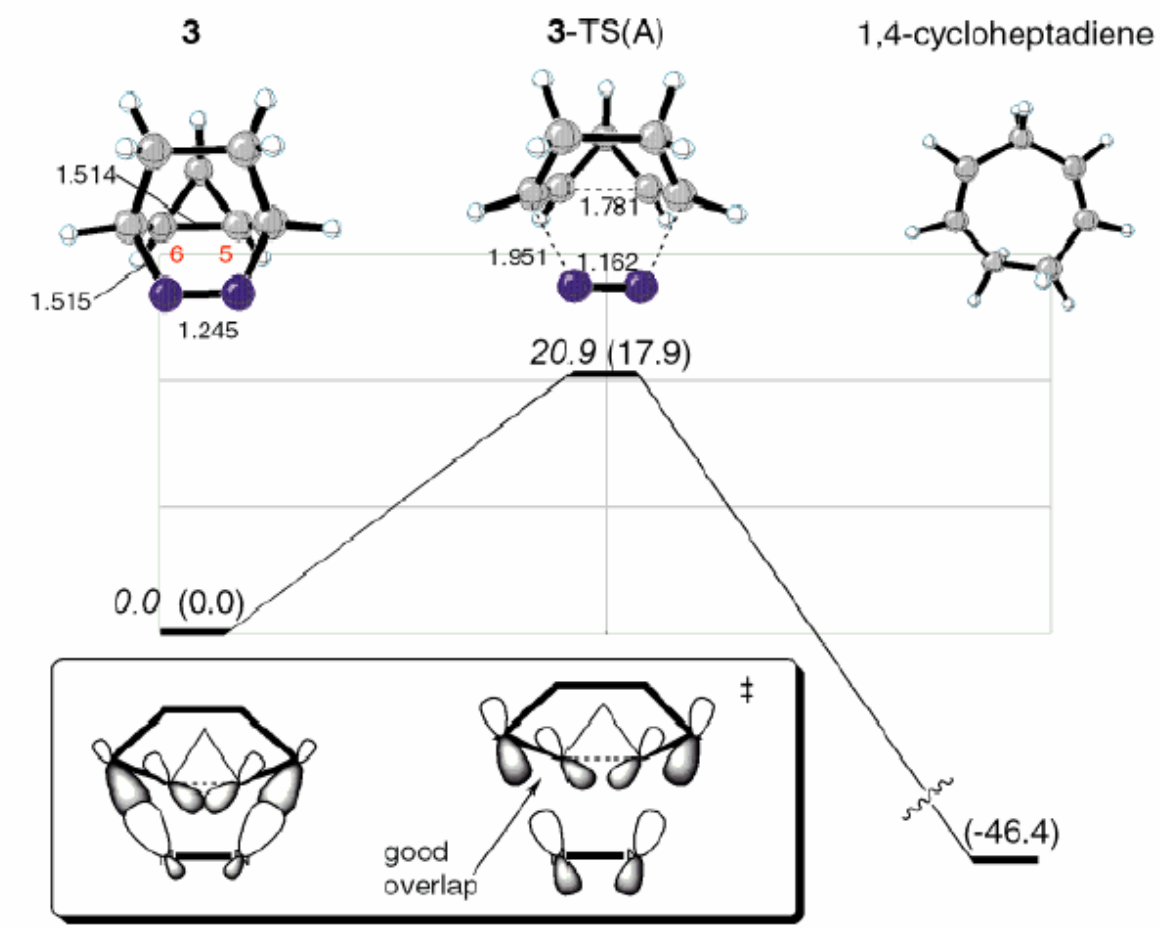

Figure 2.4.5. Geometries and energies of the reactant, products and transition state for the 1-step diazetization of $\mathrm{C}_{7} \mathrm{H}_{10} \mathrm{~N}_{2}$. The energies shown were obtained using the CASPT2 method, values in parentheses are from density functional theory calculations. From ref. 225. 


\subsubsection{Coupled-Cluster Methods.}

It is possible to show ${ }^{7}$ that the correlation energy in a many-electron system can be obtained from the result of a full CI calculation as:

$$
E_{c}=\sum_{b>a} \sum_{s>r} c_{a b}^{r s} \int d \mathbf{r} \Psi_{0} \hat{H} \Psi_{a b}^{r s}
$$

where we have used the notation of (2.4.33). The integral runs over all the electronic coordinates, and the summations are over pairs of electrons. Equation (2.4.49) can be interpreted in the following way: if we regard the inner summation as a contribution from the pair of electrons $a$ and $b$, then the total correlation energy is just the sum of the contributions from all pairs. Hence a possibility to approximate the correlation energy is to obtain the contribution of each pair independently of the other electrons, and then add the contributions. This is known as the independent electron pair approximation (IEPA), originally introduced by Sinanoğlu ${ }^{226}$ and $\mathrm{Nesbet}^{227}$. In an IEPA calculation, the contribution from each pair of electrons $a$ and $b$ is obtained separately by applying the variational principle to the pair function ${ }^{228}$ :

$$
\boldsymbol{\Psi}_{a b}=\boldsymbol{\Psi}_{0}+\sum_{s>r} c_{a b}^{r s} \boldsymbol{\Psi}_{a b}^{r s}
$$

which includes the contributions from all the excited determinants in which electrons $a$ and $b$ have been promoted to excited orbitals. By doing this variational minimization with each pair of electrons, one finds a set of pair energies, and the sum of these pair energies is an approximation to the correlation energy of the system. One important thing to note is that, although the variational principle is used in each of the pair calculations, the method is not truly variational because different contributions to the Hamiltonian are neglected in each of 
the calculations. The method, however, does not have the size extensivity problem of many variational methods like CISD.

There is a problem with the IEPA, besides the fact that it is not variational. Methods such as $\mathrm{HF}, \mathrm{CI}$ and perturbation theory yield the same correlation energy when a unitary transformation $^{229}$ is applied to the occupied spin orbitals. The IEPA method, however, does not satisfy this condition. There is a way to extend the IEPA in such a way that it is invariant under unitary transformations, however, and that also increases its accuracy significantly. This can be done by including the coupling between different electron pairs in the calculation. A way to do this, of course, would be to perform a CI calculation including the coupling between pairs, quadruples, sextuples ${ }^{230}$, etc., but the computational cost would be prohibitive. But applying the idea from the IEPA, one may assume that the coefficients $c_{a b c d}^{r s t u}$ for the quadruple excitations are not really independent from the coefficients $c_{a b}^{r s}$ (because treating the pairs as independent is not a bad approximation). This is the idea behind the Coupled-Cluster (CC) approximation, introduced by Čižek and Paldus $^{231-233}$. In a CC approximation including the double excitations (CCD), the quadruple excitation coefficients would be expressed as combinations of products of the double excitation coefficients:

$$
C_{a b c d}^{r s t u}=\sum_{p e r m}(-1)^{n p e r m} C_{i j}^{m n} C_{k l}^{o p}
$$

The summation (2.4.51) contains all possible permutations of the indices $a, b, c$, and $d$ (represented by the letters $i, j, k$ and $l$ in the summation) and $r, s, t, u$ (represented by $m, n$, $o$ and $p$ ). If the total number of permutations is even, the coefficient is 1 , and if it is odd, it is -1. For example, the coefficient of $c_{a c}^{r s} c_{b d}^{t u}$ would be -1 and the coefficient of $c_{a d}^{r u} c_{b c}^{s t}$ would be 1 . There are 18 permutations in total. This relation can be used to obtain a better approximation to the many-electron wavefunction than the one obtained using the IEPA. 
The resulting $\mathrm{CC}$ method has the advantages of being both size extensive and invariant under unitary transformations. It is however, not variational, as is the IEPA. The original formulation of the $\mathrm{CC}$ method by Čižek and Paldus was $\mathrm{CCD}^{233}$. The $\mathrm{CC}$ method was extended to include single and double excitations (CCSD) by Purvis and Bartlett ${ }^{234}$ and later expanded to include higher excitations and spin-adapted wavefunctions. More detailed description of $\mathrm{CC}$ approaches and reviews can be found in refs 5, 235 ${ }_{-}^{238}$. As with perturbation methods, it is possible to construct multi-reference CC methods using results from MCSCF methods as a starting point ${ }^{239-242}$.

General CC theories can be formulated by introducing a cluster operator $\mathbf{T}^{243}$ :

$$
\mathbf{T}=\mathbf{T}_{1}+\mathbf{T}_{2}+\cdots+\mathbf{T}_{N}
$$

where the operator $\mathbf{T}_{i}$ has the effect of generating all the $i$-th excited determinants when acting on the reference many-electron wavefunction, e.g.

$$
\mathbf{T}_{1} \boldsymbol{\Psi}_{0}=\sum_{a, r} c_{a}^{r} \boldsymbol{\Psi}_{a}^{r}
$$

In the CC literature, the coefficients $c_{a}^{r}, c_{a b}^{r s}, \cdots$ are referred to as amplitudes, and usually written using the letter $t$ instead of $c$. The coupled-cluster many-electron wavefunction is then written in terms of the cluster operator as:

$$
\boldsymbol{\Phi}_{e, c c}=e^{\mathbf{T}} \boldsymbol{\Psi}_{0}=\left(\mathbf{I}+\mathbf{T}+\frac{1}{2} \mathbf{T}^{2}+\cdots\right) \boldsymbol{\Psi}_{0}
$$


This expansion can be substituted into the electronic Schrödinger equation (2.4.4) to obtain expressions for the coefficients $c_{a}^{r}, c_{a b}^{r s}, \cdots$. Combining (2.4.52) and (2.4.54) it is possible to see an analogy between $\mathrm{CC}$ and $\mathrm{CI}$ :

$$
\boldsymbol{\Phi}_{e, c c}=e^{\mathbf{T}} \boldsymbol{\Phi}_{0}=\boldsymbol{\Phi}_{0}+\mathbf{T}_{1} \boldsymbol{\Phi}_{0}+\left(\mathbf{T}_{2}+\frac{1}{2} \mathbf{T}_{1}^{2}\right) \boldsymbol{\Phi}_{0}+\left(\mathbf{T}_{3}+\mathbf{T}_{2} \mathbf{T}_{1}+\frac{1}{6} \mathbf{T}_{1}^{3}\right) \boldsymbol{\Phi}_{0}+\cdots
$$

In truncated CC, only some terms of the cluster operator $\mathbf{T}$ are kept, for example in CCSD the expansion would be $\mathbf{T}=\mathbf{T}_{1}+\mathbf{T}_{2}$. The first two terms in the expansion (2.4.55) are analogous to the first two terms in the CI expansion (2.4.33), i.e. the HF reference and the singly excited determinants. In the second term there is already a difference. There are two terms in the double excited part: one equal to the equivalent CI term, and another counting the "disconnected" double excitations. These additional terms make the CC method size extensive even when the cluster operator is truncated, unlike what happens in CI. Just as perturbation theory includes the contributions from all the excited-determinant corrections (single, double, etc.) up to a given order, $\mathrm{CC}$ includes corrections up to a given point but to infinite order $^{235}$.

It is possible to combine perturbation and $\mathrm{CC}$ methods to obtain accurate results with a lower computational $\operatorname{cost}^{244}$. An example of this is the $\operatorname{CCSD}(\mathrm{T})$ method, where the triples contribution to the energy is estimated using perturbation theory and added to the CCSD energy.

CC methods can estimate the correlation energy very accurately, and are very frequently used to study chemical reactions. The lower-level methods CCD and CCSD have a cost proportional to $N^{6}$, and the more accurate CCSDT a cost of $N^{8}$, which precludes the use of the latter for any but very simple systems. CC theories are similar to CI and perturbation methods in the sense that the results can be improved both by adding more basis functions 
and by including more terms in the cluster expansion. In the limit of an infinite basis set with a complete (infinite) cluster expansion, the results should be exact.

Some recent representative applications involving the CC methodology are:

- A calculation of the structure, vibrational frequencies, atomization energies and standard heats of formation of hydroxylamine, ammonia, hydrogen peroxide and hyponitrous acid ${ }^{245}$ using $\operatorname{CCSD}(\mathrm{T})$ and higher level methods. Both the structural, vibrational and energy results were found to be in good agreement with available experimental results.

- A study of the energetics, geometry and vibrational frequencies of the nitric oxide dimer using singe- and multireference CC methods ${ }^{246}$. The NO dimer is a notoriously difficult molecule to study due to its very weak bonding.

- A study of the PES for the isomerization of the silicon hydrides $\mathrm{Si}_{2} \mathrm{H}_{3}$ and $\mathrm{Si}_{2} \mathrm{H}_{4}$ using the $\operatorname{CCSD}(\mathrm{T}) \operatorname{method}^{247}$. A total of 10 different isomers were found in this work. For these isomers, they obtained harmonic vibrational frequencies, dipole moments and infrared intensities. In the same work, spectroscopic measurements were made and they compared very well with the $a b$ initio results.

\subsubsection{Semi-empirical methods.}

An alternative approach to the electronic problem is provided by the semi-empirical methods. In these, the many-electron problem is simplified in some way, and then some parameters obtained from experiment or higher quality ab initio calculations are included in order to get good results. Semi-empirical methods are particularly useful for dealing with large systems (e.g. large biomolecules) where more computationally demanding methods are impossible to apply. Semi-empirical methods are not truly ab initio methods, 
because they make use of experimental information to obtain their results. Nevertheless, they play an important role in the simulation of chemical reactions, especially in complex systems.

Most semi-empirical methods can be obtained by introducing approximations into the Roothaan equations (2.4.28). There are several different levels of approximation that can be used to develop a semi-empirical method. As we mentioned in our discussion of the HF method, the largest computational cost comes from the evaluation of the two electron integrals appearing in (2.4.31):

$$
(\mu v \mid \lambda \sigma)=\iint d \mathbf{r}_{1} d \mathbf{r}_{2} \phi_{\mu}^{*}\left(\mathbf{r}_{1}\right) \phi_{v}^{*}\left(\mathbf{r}_{1}\right) \frac{1}{r_{12}} \phi_{\lambda}\left(\mathbf{r}_{2}\right) \phi_{\sigma}\left(\mathbf{r}_{2}\right)
$$

These integrals are necessary to construct the Fock operator matrix F. For a basis set with $K$ functions, there are $\sim K^{4} / 8$ distinct two-electron integrals. For a system with many electrons (e.g. a biomolecule) the cost of calculating them can be prohibitive. A way around this problem is the zero differential overlap (ZDO) approximation. Within this approximation, it is assumed that the overlap between different orbitals is always zero, i.e.

$$
\int d \mathbf{r} \phi_{\mu}^{*} \phi_{v}=0 \text { for } \mu \neq v
$$

where the integral is over any region of space. This is a stronger assumption than just saying that $S_{\mu \nu}=0$ for $\mu \neq v$, and a direct consequence of it is that the two electron integrals defined by (2.4.56) are zero unless $\mu=\nu$ and $\lambda=\sigma$. This reduces the number of distinct integrals to calculate to $\sim K^{2} / 2$, much less than in the HF method. There is, however, one major problem with the ZDO approximation. If all orbitals are assumed to have zero overlap over all space, two things happen: (1) The results may be dependent on 
the coordinate system used, because the overlap is in general not invariant to coordinate transformations, and (2) If the overlap between all pairs of orbitals in neighboring atoms is neglected, there can be no bonding.

There are several ways around the problems of the ZDO approximation. One option is used in the complete neglect of differential overlap (CNDO) method ${ }^{248,249}$. In CNDO, the twoelectron integrals $(\mu \mu \mid \lambda \lambda)$ are assumed to be independent of the shape of the orbitals $\phi_{\mu}$ and $\phi_{\lambda}$. This removes the coordinate invariance problem. If the orbitals are centered on the same atom, the corresponding integrals are assigned a value representing the average electron-electron repulsion on that atom. If they are centered on different atoms, the integrals are assigned a value representative of the average electron-electron repulsion between an electron in one atom and an electron in the other atom. Note that the latter value is also dependent on the distance between the atoms.

Two further assumptions are made in CNDO regarding the part of the Fock matrix that depends on the nuclei-electron interactions, which is given by:

$$
H_{\mu \nu} \equiv \int d \mathbf{r} \phi_{\mu}^{*}\left[-\frac{1}{2} \nabla^{2}-\sum_{I} \frac{Z_{I}}{r_{i I}}\right] \phi_{v}
$$

When $\phi_{\mu}$ and $\phi_{v}$ are on the same atom, these terms are assumed to be zero for $\mu \neq v$. For $\mu=v$, they are approximated using experimental ionization energies and electron affinities for the atom. Finally, when they are on different atoms, $H_{\mu v}$ is assumed to be proportional to the overlap integral $S_{\mu v}$, and the proportionality constants are parameterized in terms of empirical single-atom constants. 
One defect of the CNDO method is that, since two-electron integrals are assigned the same value regardless of spin, it does not consider the "exclusion principle" energy contribution. In the intermediate neglect of differential overlap (INDO) method ${ }^{250}$, these contributions are included in the form of additional contributions to the two-electron integrals that depend on the spin of the orbitals involved. This necessarily means that the differential overlap between orbitals on the same atom is not neglected anymore. In INDO, some of the two-electron integrals on the same atom are parameterized using spectroscopic data for the atom.

Aside from the CNDO and INDO methods, there are other, more sophisticated semiempirical methods. In the neglect of diatomic differential overlap (NDDO) method ${ }^{251}$, only the differential overlap between orbitals on different atoms is neglected. This means that now all the two-electron integrals $(\mu \nu \mid \lambda \sigma)$ when $\phi_{\mu}, \phi_{\lambda}$ are on the same atom and $\phi_{\lambda}, \phi_{\sigma}$ are also on the same atom have to be calculated. This causes a major increase in the computational cost for NDDO as compared to CNDO or INDO.

Other semi-empirical methods which make more use of experimental information and are more accurate than the first-generation methods discussed above are the MINDO (modified INDO) method $^{252-255}$, the MNDO (modified neglect of diatomic overlap) $\operatorname{method}^{256,257}$, the AM1 (Austin Model 1, a second-generation MNDO) method ${ }^{258}$, the PM3 (Parameterized Model 3, a third-generation MNDO) method ${ }^{259,260}$, and the SAM1 (Semi Ab initio Model 1) method $^{261}$, among others.

It is not easy in general to assess the relative accuracy of all the available semi-empirical methods, mainly because their accuracy depends on what system they are applied to. Some comparisons of the strengths and weaknesses of various empirical methods as applied to various systems can be found in refs. 5, 262-265. 
As it is the case with force-field methods, there is no way to systematically improve on the results obtained with a semi-empirical method. Also similarly, their performance is limited to compounds for which experimental data is available; unlike $a b$ initio methods, it is not generally good to use a semi-empirical method to predict the properties of a new or unknown compound. Most applications of semi-empirical methods involve the study of very large molecules, or molecules in complex environments. Some recent representative applications of semi-empirical methods include:

- A calculation of the permanent dipole of the enzyme $\alpha$-chymotrypsin using the AM1, PM3 and PM5 methods ${ }^{266}$. The results from the PM5 method were the closest to the experimental values for this particular system.

- A study of the reaction path for the reduction of nitric oxide in the enzyme nitric oxide reductase from the fungus Fusarium oxysporum using the SAM1 method ${ }^{267}$. The reaction path obtained for this process consisted of a cycle of seven reaction steps, and was similar to a mechanism proposed based on previous experimental results.

- A study of the fluorination ${ }^{268}$ and the hydrogenation and oxidation ${ }^{269}$ of carbon nanotubes using the MNDO method. In these works the potential energy curve for the adsorption of fluorine, hydrogen and oxygen was obtained for different kinds of nanotubes.

\subsubsection{Density Functional Theory. ${ }^{3-5,9,270-274}$}

As shown in the previous sections, an accurate estimation of the electronic energies for a given atom or molecule is in general a very computationally expensive task. The relatively simple approach of the HF method suffers from the correlation energy error, and improving on HF results is often very computationally demanding. Density functional theory (DFT) is an alternative approach that permits one to obtain reasonably accurate results for complex systems. 
The main difference between DFT and the methods mentioned in the previous sections is that the fundamental variable is not the wave function but the electronic density $\rho(\mathbf{r})$ defined by:

$$
\rho\left(\mathbf{r}_{i}\right)=N \int \cdots \int\left|\Phi_{e}\right|^{2} d \mathbf{r}_{1} d \mathbf{r}_{2} \cdots d \mathbf{r}_{i-1} d \sigma_{i} d \mathbf{r}_{i+1} \cdots d \mathbf{r}_{N}
$$

In (2.4.59), $N$ is the number of electrons and $\sigma_{i}$ is the spin coordinate of electron $i$. The results that make DFT possible are two theorems proved by Hohenberg and Kohn in $1964^{275,276}$, and later generalized by several authors. The first theorem states that, if one knows the electronic density $\rho(\mathbf{r})$ for a given system, then all the properties of the ground state can be obtained. The second theorem is a variational principle for the electronic density. It states that, for any electronic density $\rho$, which may or may not be the true density for the ground state, the relation $E[\rho] \geq E_{0}$ is satisfied, where $E_{0}$ is the energy of the ground state. This is analogous to the variational principle for the wavefunction mentioned in section 2.4.2, and it also leads to a method for estimating $E_{0}$.

The energy functional $E[\rho]$ in DFT can be expressed as:

$$
E[\rho]=T[\rho]+V_{e n}[\rho]+V_{e e}[\rho]
$$

where $T[\rho]$ accounts for the kinetic energy, $V_{e n}[\rho]$ for the potential energy of the electron-nucleus interaction, and $V_{e e}[\rho]$ for the potential energy of the electron-electron interaction. The later is further decomposed into:

$$
V_{e e}[\rho]=V_{\text {coul }}[\rho]+V_{x c}[\rho]
$$


where $V_{\text {coul }}[\rho]$ is the potential energy of the Coulomb interaction and $V_{x c}[\rho]$ is the called the exchange-correlation potential energy. Comparing equations (2.4.60) and (2.4.61) with equations (2.4.24)-(2.4.27) shows an analogy between HF and DFT, and also the main difference: the correlation energy is included in the term $V_{x c}[\rho]$ together with the exchange potential, whereas in HF only the exchange part is accounted for. Also, the exchange energy in HF is calculated exactly, whereas in DFT it is normally approximated in some way.

If the form of the energy functional $E[\rho]$ is known, the problem of finding the groundstate electronic density and its energy is reduced to a minimization problem through use of the variational principle $E[\rho] \geq E_{0}$. It is possible to simplify the problem further. Since the electronic density determines the ground state, any system with the same electronic density as the real system will have the same ground-state properties. One may then think of a set of noninteracting electrons that give the same electronic density as the real system. In this case, the exchange-correlation energy in terms of the wavefunctions of these noninteracting electrons has to contain all the missing terms. In particular, it will also contain a correction to the kinetic energy. This approach, known as the Kohn-Sham ${ }^{277,278}$ method, is sketched in figure 2.4.6.

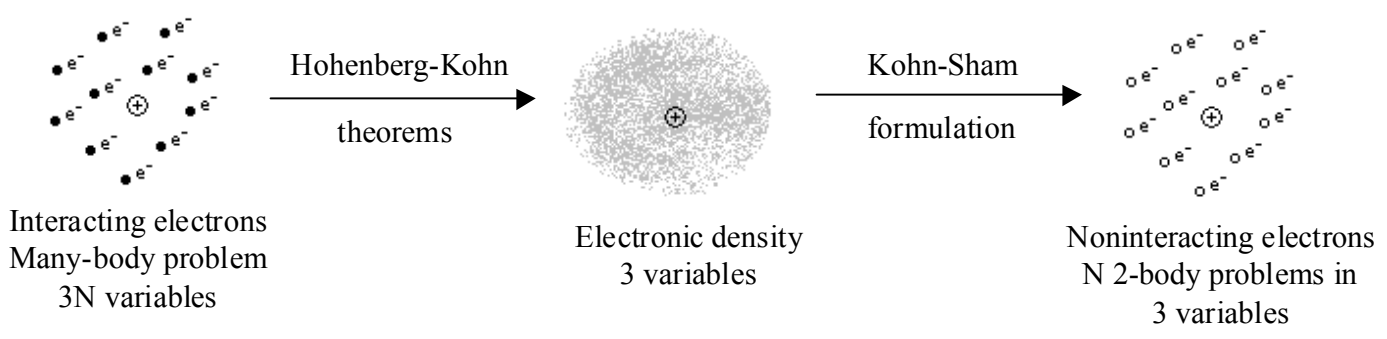

Figure 2.4.6. The Kohn-Sham approach. 
Within this framework, one may write the electronic density as a sum over occupied orbitals:

$$
\rho(\mathbf{r})=\sum_{i=1}^{N_{\text {orb }}}\left|\psi_{i}(\mathbf{r})\right|^{2}
$$

and the expressions for $T[\rho], V_{e n}[\rho]$ and $V_{\text {coul }}[\rho]$ can be written as:

$$
\begin{gathered}
T(\rho)=2 \sum_{i=1}^{N_{\text {orb }}} \int d \mathbf{r} \psi_{i}^{*}\left(-\frac{1}{2} \nabla^{2} \psi_{i}\right) \\
V_{\text {en }}[\rho]=\sum_{i=1}^{N_{\text {orb }}} \int d \mathbf{r} \psi_{i}^{*}\left(-\sum_{I} \frac{Z_{I}}{r_{i I}}\right) \psi_{i}=-\sum_{I} \int d \mathbf{r} \rho(\mathbf{r}) \frac{Z_{I}}{\left|\mathbf{r}-\mathbf{r}_{I}\right|} \\
V_{\text {coul }}[\rho]=\sum_{i=1}^{N_{\text {orb }}} \sum_{j>i}^{N_{\text {ort }}} \iint d \mathbf{r}_{1} d \mathbf{r}_{2} \psi_{i}^{*}\left(\mathbf{r}_{1}\right) \psi_{j}^{*}\left(\mathbf{r}_{2}\right) \frac{1}{r_{12}} \psi_{i}\left(\mathbf{r}_{1}\right) \psi_{j}\left(\mathbf{r}_{2}\right)= \\
=\frac{1}{2} \iint d \mathbf{r}_{1} d \mathbf{r}_{2} \frac{\rho\left(\mathbf{r}_{1}\right) \rho\left(\mathbf{r}_{2}\right)}{r_{12}}
\end{gathered}
$$

For the exchange-correlation potential $V_{x c}[\rho]$, however, an additional theory is necessary. The simplest assumption is the local density approximation (LDA), which states that the exchange-correlation energy is locally that of a uniform electron gas; i.e. the exchange correlation energy density at a position $\mathbf{r}$ is the same as that of a uniform electron gas of density $\rho$, where $\rho$ equals the local electron density at $\mathbf{r}$ in the actual system. If the exchange correlation energy $E_{x c}(\rho)$ for a uniform electron gas is known as a function of the density $\rho$, then $V_{x c}[\rho]$ can be obtained from:

$$
V_{x c}[\rho]=\int d \mathbf{r} \rho(\mathbf{r}) E_{x c}[\rho(\mathbf{r})]
$$


In formulations of DFT for open-shell systems, or systems with spin polarization, the electronic density is treated differently for spin-up and spin-down electrons. In this case one speaks of the local spin density (LSD) approximation, instead of the LDA.

There are more elaborate expressions for the exchange-correlation energy that give better results than the LDA. One of the most common approaches is to also include a dependence of the exchange-correlation energy on the gradient of the electronic density. This leads to the generalized gradient approximation ${ }^{279-284}$ (GGA). Among the most commonly used functionals of this kind are the Becke ${ }^{280}-$ Perdew $^{285}$ (BP) functional, the Perdew-Wang $1991^{283,286-288}$ (PW91) functional, the Becke ${ }^{280}$-Lee-Yang-Parr ${ }^{289}$ (BLYP) functional, and the Perdew-Burke-Ernzerhof ${ }^{281}$ (PBE) functional. Some new GGA functionals that seem to perform very well ${ }^{290-292}$ are the OLYP functional, a combination of the OPTX exchange functional of Handy and Cohen ${ }^{293-295}$ and the Lee-Yang and Parr correlation functional, and the Hamprecht-Cohen-Tozer-Handy (HCTH) functionals ${ }^{296-298}$. Other exchangecorrelation functionals in common use include a contribution from exact exchange, i.e. exchange energy calculated as in HF theory. A functional of this kind, which is very commonly used, is the three-parameter Becke-Lee-Yang-Parr (B3LYP) functional ${ }^{299}$. The main drawback of these functionals is that the calculation of the exact exchange energy is very computationally demanding, thus restricting somewhat the system sizes that can be handled.

Once the exchange-correlation functional is defined, a DFT calculation proceeds by solving the one-electron Kohn-Sham equations ${ }^{277,278}$ :

$$
\hat{E}_{K S} \psi_{i}=\varepsilon_{i} \psi_{i}
$$


where $\hat{E}_{K S}$ is the one-electron Kohn-Sham operator:

$$
\hat{E}_{K S}=-\frac{\nabla^{2}}{2}-\sum_{m} \frac{Z_{m}}{\left|\mathbf{r}-\mathbf{r}_{m}\right|}+\int d \mathbf{r}^{\prime} \frac{\rho\left(\mathbf{r}^{\prime}\right)}{\left|\mathbf{r}-\mathbf{r}^{\prime}\right|}+\frac{\delta V_{x c}}{\delta \rho}
$$

In (2.4.68), $\delta$ denotes functional differentiation. Once this system of equations is set up the rest of the procedure is very similar to a HF calculation, and actually less computationally demanding. Expanding now the $\psi_{i}$ in terms of some basis functions as in (2.4.13) one can write a system of equations for the coefficients $C_{\mu i}$ and obtain the electronic density and the orbital energies.

The fact that DFT allows the calculation of energies with a much lower computational cost than other $a b$ initio approaches makes this method one of the most popular. DFT allows studies of much larger and more complex systems than most other methods, and with the newer functionals its accuracy can be comparable to that of much more expensive methods.

One drawback of DFT is the fact that it is a method designed to obtain only ground-state properties. There are, however, extensions of DFT that allow the treatment of excited states, such as the restricted open shell Kohn-Sham (ROKS) method ${ }^{300,301}$, which uses a mixture of symmetry-adapted Kohn-Sham all-electron wavefunctions, the density functional theory/single configuration interaction (DFT/SCI) method ${ }^{302,303}$, which mixes the ideas of the CI method and DFT, and the time-dependent density functional theory (TDDFT) method ${ }^{304-306}$, which so far seems the most promising extension of DFT to excited states. Another problem of DFT is, of course, that its accuracy is only as good as the exchange-correlation functional used. The Hohenberg-Kohn theorem guarantees that the DFT treatment is exact, unlike semiempirical approaches, because there exists an 
exchange-correlation functional that makes its results exact. But this functional is not known and we are forced to approximate it in some way. Other than employing a larger basis set, as with HF, there is no evident way to systematically improve on the results: one may try two different exchange-correlation functionals for the same system and there is in principle no way to tell which result is better unless results from a higher-level $a b$ initio calculation are available, and this is usually not the case. Nevertheless, DFT has an excellent accuracy/computational cost ratio, and this makes it an extremely useful tool in quantum chemistry. A detailed treatment of DFT can be found in refs. 3-5,270-273.

There are numerous applications of DFT in the literature, and their number is likely to increase as better exchange-correlation functionals are developed. DFT is used often to study large molecules, surface chemistry and properties of solids. Another common application is to do geometry optimizations that are later followed by energy calculations using more accurate methods. Some recent representative applications include:

- A study of the reaction mechanism of the cycloaddition of 1,5-cyclooctadiene with alkynes over a ruthenium catalyst, using the B3LYP functional ${ }^{307}$. In this work, several possible mechanisms for this complex reaction were considered, something made possible by the relatively low computational cost of DFT.

- An analysis of the stability of carbon dioxide hydrates with 20, 24 and 28 water molecules using DFT with the B3LYP functional ${ }^{308}$. In this study it was found that the tetrakaidecahedral cluster with 24 molecules was the most stable of the ones considered, and good agreement with experimental results was obtained.

- A study of the vanadyl pyrophosphate (100) surface and its ability to oxidize $n$-butane to maleic anhydride using the PW91 functional ${ }^{309}$. In this work the chemisorption of water onto the surface and its role on the selective oxidation of $n$-butane was analyzed. 


\subsubsection{Ab Initio Molecular Dynamics $4,11,181,310-313$}

In order to simulate chemically reactive systems, one often needs to do first an $a b$ initio calculation in order to find the potential energy surface. The results of this $a b$ initio calculation can then be fitted to an empirical equation for the potential energy and this in turn can be used in a simulation within one of the techniques discussed in the following sections. It is also possible, in principle, to omit the fitting stage and do the $a b$ initio calculations "on-the-fly" to obtain the potential energies within the simulation. There are several ways that this can be done. One may, for example, run a Molecular Dynamics (MD) simulation in which the energies at each time step are found by minimizing the electronic energy. This approach is sometimes called Born-Oppenheimer Molecular Dynamics $^{181,314}$ (BOMD). The major difficulty with BOMD is that it is necessary to perform an extremely accurate $a b$ initio calculation at each time-step, because otherwise the error accumulates and causes an unphysical drift of the total energy of the system.

Car and Parrinello ${ }^{315}$ proposed in 1985 an alternative way to obtain ab initio energies "onthe-fly", by embedding the dynamics of the electrons in the MD Lagrangian. This is called the Car-Parrinello Molecular Dynamics (CPMD) method. Although in principle one may formulate CPMD with any ab initio method, DFT is the most commonly used because of its very good accuracy/computational cost ratio.

The idea behind CPMD is to simulate simultaneously the motion of the nuclei and of the electrons. Nevertheless, since the electrons cannot be treated classically, what is really done is to generate a fictitious dynamics of the one-electron orbitals $\psi_{i}$. 
The first step to generate the dynamic equations for both the nuclei and the electrons in CPMD is to define a Lagrangian comprising both the electronic and nuclear dynamics as $^{181,310,315}$ :

$$
L=\frac{1}{2} \sum_{i=1}^{N} \mu_{i} \int d \mathbf{r}\left|\dot{\psi}_{i}(\mathbf{r})\right|^{2}+\frac{1}{2} \sum_{I} M_{I} \dot{\mathbf{R}}_{I}-E\left[\psi_{i}, \mathbf{R}_{I}\right]+\sum_{i=1}^{N} \sum_{j=1}^{N} \Lambda_{i j}\left(\left\langle\psi_{i} \mid \psi_{j}\right\rangle-\delta_{i j}\right)
$$

In (2.4.69), the $\mu_{i}$ are fictitious masses assigned to the electron orbitals (typically between 400 and 2400 a.u.), $M_{I}$ and $\mathbf{R}_{I}$ are the mass and the position of nucleus $I, E$ is the expectation value of the Kohn-Sham Hamiltonian, $\delta_{i j}$ is the Kronecker delta and the $\Lambda_{i j}$ are Lagrange multipliers associated with the orthonormality constraints ${ }^{316}$ :

$$
\left\langle\psi_{i} \mid \psi_{j}\right\rangle=\int d \mathbf{r} \psi_{i}^{*} \psi_{j}=\delta_{i j}
$$

The equations of motion for the nuclei and the electronic orbitals can be obtained by writing the Euler-Lagrange equations associated with the Lagrangian (2.4.69):

$$
\begin{gathered}
\mu_{i} \ddot{\psi}_{i}=-\frac{\delta E\left[\psi_{i}, \mathbf{R}_{I}\right]}{\delta \psi_{i}^{*}}+\sum_{j=1}^{N} \Lambda_{i j} \psi_{j} \\
M_{I} \ddot{\mathbf{R}}_{I}=-\frac{\partial E\left[\psi_{i}, \mathbf{R}_{I}\right]}{\partial \mathbf{R}_{I}}
\end{gathered}
$$

These equations can be integrated using a Verlet ${ }^{317}$ or velocity Verlet $^{318,319}$ algorithm $^{11,12}$ or a similar numerical technique ${ }^{320-322}$. It is important, however, to keep the electron dynamics separated from the nuclear dynamics, i.e. to keep the fictitious electronic kinetic energy low, in order to keep the system on the Born-Oppenheimer surface. In some cases, such as the simulation of non-conducting molecules, this can be accomplished simply by 
using a large enough value for the orbital masses $\mu_{i}$. For conducting molecules, however, since the valence electrons are less tightly bound to the nuclei, there is a tendency for the nuclei and the electrons to achieve thermal equilibrium, hence removing the system from the Born-Oppenheimer surface. This may be avoided by using Nose-Hoover ${ }^{323-325}$ thermostats $^{11,12}$ for the nuclei and the electrons ${ }^{181,326,327}$.

The difficult part in the solution of the system (2.4.71)-(2.4.72) is usually the integration of (2.4.71), the equation of motion for the orbitals subject to the orthonormality constraints (2.4.70). In the standard Verlet scheme, which is the most commonly used, an unconstrained set of orbitals $\hat{\psi}_{i}$ at $t+\delta t$ is found from:

$$
\hat{\psi}_{i}(t+\delta t)=2 \psi_{i}(t)-\psi_{i}(t-\delta t)-\left.\frac{\delta t^{2}}{\mu} \frac{\delta E\left[\psi_{i}, \mathbf{R}_{I}\right]}{\delta \psi_{i}^{*}}\right|_{t}
$$

And then these predicted orbitals are corrected by adding in the effect of the constraints:

$$
\psi_{i}(t+\delta t)=\hat{\psi}_{i}(t+\delta t)+\sum_{j=1}^{N} \frac{\delta t^{2}}{\mu_{i}} \Lambda_{i j} \psi_{j}(t)
$$

In order to find the Lagrange multipliers $\Lambda_{i j}$, equation (2.4.74) is substituted into the constraints (2.4.70) to get a system of equations that can be solved iteratively. Once the $\Lambda_{i j}$ are known, the correct orbitals are obtained from (2.4.74). For a more detailed description of this kind of approach see refs. ${ }^{181},{ }^{320}$. Combining the Verlet algorithm with the constraints in this fashion is the method known as the SHAKE algorithm ${ }^{328}$. A similar method that uses the velocity Verlet method is known as the RATTLE algorithm ${ }^{329}$. 
One advantage of the CPMD approach as compared to BOMD is that it is more numerically stable. As mentioned above, BOMD requires a very accurate minimization of the electronic energy at each step to avoid energy drifts. CPMD does not have this problem, the total energy usually oscillates and its average remains constant ${ }^{310,311}$. On the other hand, doing a full minimization of the electronic energy at each timestep allows BOMD to use larger timesteps than CPMD (usually $~ 10$ times larger) because they only need to be of the order of the characteristic time of the nuclear motion. The extra computational effort associated with the accurate $a b$ initio calculations, however, counterbalances this advantage, and at the end the choice depends more on how much of an energy drift can be accepted as payment for a faster calculation. A discussion of the relative computational efficiencies of CPMD and BOMD can be found in ref. 181. Recent reviews on the CPMD method can be found in refs. $312,313$.

Another advantage of CPMD is that treating the dynamics of the nuclei and the electrons simultaneously yields a very good picture of the real behavior of the system. It is not necessary to introduce empirical equations for the intermolecular potential, at least in principle, because it is obtained during the simulation. This is of course true as long as the relevant interactions are accurately pictured by the $a b$ initio method within. With DFT, for example, dispersion interactions are not well represented unless the nuclei are close to each other, limiting the application of the method in cases where dispersion is the most important interaction ${ }^{330}$. In a case like this, better results may be obtained by treating the interactions at longer distances with an empirical potential and using $a b$ initio methods at shorter distances, as in QM/MM.

The other major issue with CPMD is its computational cost when compared to "classical" molecular simulation techniques that use empirical potentials. The time-step in a CPMD simulation has to be kept small enough to get good statistics for the motion of the electrons, typically of the order of 0.1 fs. Sometimes it is possible to increase the orbital 
mass $\mu$ and use slightly larger time-steps and still get good statistics. However, the total

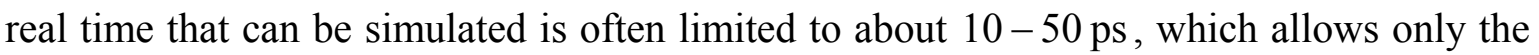
study of very fast processes. In addition, the size of the system that can be studied is limited, usually of the order of 100 heavy atoms. The time scale problem can be overcome by combining CPMD with a multiple time-scale technique such as the ones described in the next section; some applications of this idea will be discussed in section 2.5.

CPMD has also been adapted to efficiently explore potential energy surfaces to look for different transition states and products in complex reactions by including historydependent terms into the Lagrangian, as in the Laio-Parrinello Metadynamics method $^{331,332}$. This technique seems very close to being a "black-box" method for studying reaction mechanisms. It is very useful, as there are many reactions for which both the reaction mechanism and the possible products are not evident a priori.

Although molecular dynamics is most commonly combined with DFT due to its high accuracy/computational cost ratio, it has also been combined with other ab initio methods. In the literature there are examples combining molecular dynamics with the HF method ${ }^{333-}$ 335 , the CI method ${ }^{336}$, the CASSCF method ${ }^{337,338}$ and others ${ }^{339,340}$.

Applications of $a b$ initio molecular dynamics have increased recently due to the availability of more efficient algorithms and faster computers. Some recent representative applications include:

- A CPMD calculation of the infrared spectrum and other properties of uracil in an aqueous solution ${ }^{341}$, showing a remarkably good agreement with experiment. The CPMD method is often used to study water and aqueous solutions, as well as other hydrogen-bonded liquids ${ }^{342}$. Since the main interactions in water and aqueous solutions are hydrogen bonding and Coulomb interactions, which are strong, they can be 
pictured fairly well by using DFT. A recent study assessing the ability of CPMD to

simulate water can be found in ref. ${ }^{343}$, and some other recent applications are in refs. $344-348$.

- A study of the solvent effects on the ground state and first excited singlet state of acetone in aqueous solution, using a new hybrid Car-Parrinello QM/MM technique ${ }^{349}$. In this work the radial distribution function of the water atoms around the carbonyl oxygen is obtained for both the ground state and the excited state, and the absorption and fluorescence spectra of acetone in both the gas phase and the aqueous phase are calculated. The results obtained are in reasonably good agreement with experimental data for this system.

- A study of the torsions of two model arylamides, acetanilide and $O$ methylthioacetanilide, using $\mathrm{CPMD}^{350}$. The dynamics of these molecules are interesting because they are capable of forming intramolecular hydrogen bonds.

\subsubsection{Quantum mechanics/molecular mechanics, ${ }^{4,531-367}$.}

As was discussed in section 2.3.1, a purely FF approach is adequate mostly for studying reactions that involve conformational changes, because force fields are classical models. Reactions that involve bond breaking or formation are much more difficult to represent, since this is inherently a quantum mechanical process. Although there are recently developed force fields targeted at the description of these reactions, such as $\mathrm{RFF}^{110}$, AIREBO $^{108}$ and $\operatorname{ReaxFF}^{61,69}$, it is in general preferable to use an electronic structure method to study them. The problem is that for many systems of interest an electronic structure method can have a prohibitive computational cost. It is possible to find a compromise between the two approaches by using a quantum chemistry method to handle the site(s) in the system where bond breaking or formation occurs, and a FF method to describe the other interactions. This approach is known as the quantum mechanics/molecular mechanics (QM/MM) method ${ }^{368,369}$. 
The main idea in $\mathrm{QM} / \mathrm{MM}$ methods is to partition space into several regions. In the simplest case, the system would be separated into a quantum mechanical (QM) region, where an electronic structure method would be used to obtain the energies ${ }^{370}$, a molecular mechanical (MM) region, represented by a FF, and possibly a boundary region. The major difficulty in the QM/MM approach is to "match" the QM and MM potentials in a smooth way. It is also not trivial in general to decide which parts of the system would be in which region. For a system with the three regions described, the Hamiltonian of the system could be written as ${ }^{371}$ :

$$
H=H_{Q M}+H_{M M}+H_{Q M / M M}
$$

As depicted in figure 2.4.7, the term $H_{Q M}$ includes the interactions between particles in the QM region, and $H_{M M}$ includes the interactions between particles in the MM region. Both of this terms can be directly taken from the particular electronic structure method and FF used for each region. The $H_{Q M / M M}$ term includes the interactions between particles in the QM and MM regions of the system. This is the part that is difficult to model, and its particular form will depend on the electronic structure method chosen for the QM part and the FF used for the MM part. A detailed discussion on how to build the QM/MM Hamiltonian for different methods is outside the scope of this work, more detailed descriptions and references can be found in refs. 351-358.

It is possible to include more than one $\mathrm{QM}$ or $\mathrm{MM}$ region in a $\mathrm{QM} / \mathrm{MM}$ calculation. For example, a very high-level $a b$ initio method can be used in a small region, and a lower level method in the region surrounding it. Or a more complex FF can be used in an intermediate region and a simpler one in the outer region. This can be done, for example, in the ONIOM (Our own N-layered Integrated $\mathrm{mO}$ and $\mathrm{mM}$ ) $\operatorname{method}^{372,373}$. A generalpurpose implementation of QM/MM has been recently developed by the QUASI 
(QUAntum Simulation in Industry) project, a description of this can be found in ref. 374.

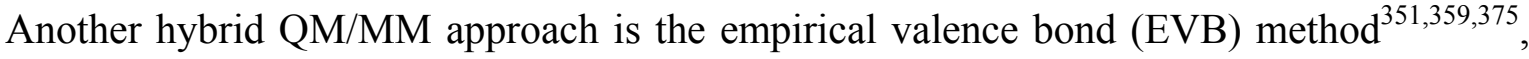
which is based on the valence bond representation rather than the molecular orbital representation of the wavefunction.

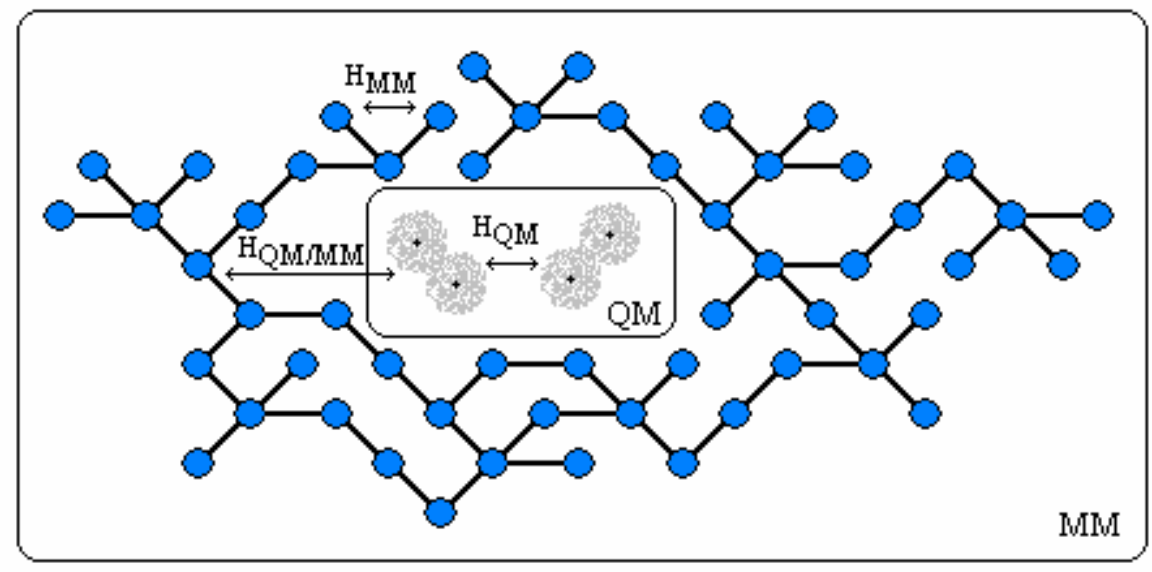

Figure 2.4.7. Sketch of a $Q M / M M$ system, showing the kinds of interactions that contribute to each part of the Hamiltonian in equation (2.4.75)

Applications of QM/MM methods are common in the study of solvation effects, reactions involving macromolecules (particularly enzymes), and surface chemistry. The QM/MM methodology seems to be an extremely efficient way of handling complex problem in chemistry. There are many examples of applications of $\mathrm{QM} / \mathrm{MM}$ in the literature, and the number is likely to grow as the available computational power increases and the techniques are refined due to the high accuracy/computational cost ratio of the method. Some recent examples showing applications of the $\mathrm{QM} / \mathrm{MM}$ methodology include:

- A study of the catalytic mechanism of the wild type and several mutants of the human enzyme cyclophilin A for the proline isomerization reaction ${ }^{376}$. In this work the 
contributions of various effects to the catalytic effect of the enzyme are quantified, and transition states are found for both the wild type and the mutants.

- An analysis of the energetics of eight enol isomers of malonaldehyde in carbon tetrachloride solution ${ }^{377}$, showing the changes in the relative energies and conformations, as well as the excitation energies of the isomers due to the interactions with the solvent.

- The original paper of the QUASI ${ }^{374}$ program shows several applications to catalysis and enzyme chemistry, including a study of the mechanism of the synthesis of methanol over a $\mathrm{Cu} / \mathrm{ZnO}$ catalyst, a study of the decomposition of nitrous oxide on a $\mathrm{Cu}$-exchanged zeolite, and reactions in triosephosphate isomerase, p-hydroxybenzoate hydroxylase and cytochrome P450 enzymes.

\subsection{Classical Simulation Methods for Equilibrium and Rate Constants.}

As we mentioned in section 2.2, the goal of a simulation method for chemical reactions is usually to estimate an equilibrium constant or a reaction rate. Equilibrium constants are easier because they only require the knowledge of the thermodynamic properties of the reactants and the products. For kinetic rates the problem is more complicated. In the following sections we will discuss some of the techniques that can be used to obtain equilibrium and rate constants once the PES is known (or a method for obtaining it has been chosen). In section 2.5.1 we will discuss an approach for the estimation of equilibrium constants, the reactive Monte Carlo method. In the remaining sections we will discuss some of the methods that can be used for estimating reaction rates.

It is important to note that, although the methods in these sections are usually termed "classical", this does not mean that no quantum mechanical calculations are involved. $A b$ initio methods are still necessary to obtain the PES that will later be used to estimate 
reaction rates (unless a purely FF description is used). The term "classical" is used to distinguish the methods that are mostly based in classical statistical mechanics from those where the description of the reactive process itself is quantum mechanical (see the discussion at the end of section 2.2).

Another important thing to keep in mind is that the accuracy of the results is determined by the method used to generate the PES (sections 2.3 and 2.4). If the $a b$ initio (or FF) method chosen does not give a good representation of the system, the results will be bad regardless of how good is the method chosen to determine the rate or equilibrium constant.

\subsubsection{Estimation of Equilibrium Constants ${ }^{3}$}

The equilibrium constant for a given chemical reaction is a thermodynamic equilibrium property, and hence it depends only on the thermodynamic properties of the reactants and the products involved, and not on the reaction mechanism. This can be restated in the terms of section 2.3 by saying that the equilibrium constant depends only on what happens at the minima of the PES or, at a finite temperature, the minima of the free energy surface (FES). From classical thermodynamics, we know that the equilibrium constant can be expressed as:

$$
K_{e q}=\exp \left(-\Delta G^{0} / k T\right)=\prod_{i} a_{i}^{v_{i}}
$$

where $\Delta G^{0}$ is the change in the Gibbs free energy per molecule for the reaction with all the components in their standard states, the $v_{i}$ are the stoichometric coefficients and the $a_{i}$ are the activities of the components involved in the reaction. These quantities can be related to the microscopic properties of the system through statistical mechanics. For 
example, for reacting mixture of ideal gases it is possible to write the equilibrium constant in terms of the molecular partition functions as ${ }^{3,378}$ :

$$
K_{e q}^{i d}=\prod_{i}\left(q_{i} / V\right)^{v_{i}}
$$

where the product runs over all components and the $q_{i}$ are the molecular partition functions. Within the semiclassical approximation ${ }^{379}$ it is possible to rewrite these partition functions as products of contributions from the different degrees of freedom ${ }^{380}$ :

$$
q_{i}=q_{i, t} q_{i, r} q_{i, v} q_{i, e} q_{i, i}
$$

In (2.5.3), $q_{i, t}=V / \Lambda^{3}$ is the translational partition function ( $\Lambda$ being the thermal De Broglie wavelength), and the other terms are respectively the rotational, vibrational, electronic and internal partition function. The latter corresponds to internal degrees of freedom not considered in the remaining terms (e.g. internal rotations about a bond). Depending on the temperature, the rotational degrees of freedom may be treated classically or quantum mechanically. The vibrational and electronic partition functions must be obtained from quantum mechanics unless the system is at very high temperatures (in which case the semiclassical approximation will probably not work anyway). The electronic partition function is in many cases assumed to be constant, as we mentioned in the introduction, because for a typical system at a near-ambient temperature practically all the molecules will be in the electronic ground state. If this is not the case, it is necessary to obtain the energies for the excited electronic states. In order to obtain the vibrational partition function it is necessary to know the characteristic frequencies of oscillation for the given molecule; these can be obtained by doing a vibrational analysis using linear response theory with an $a b$ initio method (see, for example, ref. 272). 
When the mixture is not ideal, it is necessary to include the effect of the intermolecular potential energy in the calculation of the equilibrium constant. Several attempts have been made to estimate equilibrium constants for chemical reactions in non-ideal systems by molecular simulation. In 1981, Coker and Watts ${ }^{381,382}$ proposed a modification of the Grand Canonical Monte Carlo ${ }^{383-385}$ (GCMC) method ${ }^{11,12}$ to obtain equilibrium constants. In the method an extra Monte Carlo move was added that swapped the identity of a reactant molecule with that of a product molecule, and the transition probability was obtained by using the difference between the chemical potential of the two species. They applied their method to the reaction $\mathrm{Br}_{2}+\mathrm{Cl}_{2} \rightarrow 2 \mathrm{BrCl}$ and obtained very good agreement with experimental results. The main drawback of the method, aside from requiring the chemical potentials as an input, is the fact that it can only be used to simulate equimolecular reactions. This method was later modified and extended by Kofke and Glandt $^{386}$, who called the new method the semigrand canonical Monte Carlo (SGCMC) method. The SGCMC method, although more versatile than the original modified GCMC (allowing, for example, for studying phase and chemical equilibria simultaneously), still had the limitation of being only applicable to equimolecular reactions. After this work, several other methods were proposed for the simulation of chemical equilibrium, such as the $\mathrm{N}_{\text {atoms }} \mathrm{P}-\mathrm{T}$ ensemble method of Shaw ${ }^{387}$, the association biased Monte Carlo method $^{388,389}$, the bond-biased Monte Carlo method ${ }^{390}$, and the subspace sampling Monte Carlo method ${ }^{391-393}$. All of these methods were successful for the systems studied, yet were difficult to generalize to any kind of reaction or ensemble, or required not readily available parameters $^{394}$.

Another method for the simulation of chemical equilibrium, the reactive Monte Carlo (RxMC) method, was developed independently by Smith and Triska ${ }^{395}$ and Johnson et al. ${ }^{396}$. The RxMC method is similar to the $\mathrm{N}_{\text {atoms }}-\mathrm{P}-\mathrm{T}$ ensemble method of Shaw, but is readily adapted to reactions where the number of moles is not preserved. 
A simulation using RxMC includes "reactive" moves that may correspond to forward or backward reaction steps. In order to preserved detailed balance, both types of moves have to be attempted with equal probability ${ }^{395,397}$. In a forward reaction step, reactant molecules are removed and replaced with product molecules according to the stoichiometry of the reaction. The acceptance probability of this move is obtained from:

$$
P_{a c c}=\min \left[1, \exp \left(-\frac{\delta \mathcal{U}}{k T}\right) \prod_{i} q_{i}^{v_{i}} \frac{N_{i} !}{\left(N_{i}+v_{i}\right) !}\right]
$$

where $\delta \mathcal{U}$ is the change in the potential energy between the initial and final configurations. For a reverse reaction step, the product molecules are removed and replaced with reactant molecules. It is important to do so in a way that preserves the microscopic reversibility. For example, when simulating a reaction of the form $\mathrm{A}+\mathrm{B} \rightarrow \mathrm{C}+\mathrm{D}$, a forward reaction step may be replacing a molecule of $\mathrm{A}$ with a molecule of $\mathrm{C}$ and a molecule of $\mathrm{B}$ with a molecule of $\mathrm{D}$. If this is so, then in the reverse reaction step $\mathrm{C}$ has to be replaced with $\mathrm{A}$ and $\mathrm{D}$ with $\mathrm{B}$ to preserve the microscopic reversibility. The acceptance probability for a reverse reaction step is given by:

$$
P_{a c c}=\min \left[1, \exp \left(-\frac{\delta \mathcal{U}}{k T}\right) \prod_{i} q_{i}^{-v_{i}} \frac{N_{i} !}{\left(N_{i}-v_{i}\right) !}\right]
$$

The acceptance criteria (2.5.4) and (2.5.5) can be equivalently formulated in terms of the Gibbs free energy of reaction instead of the molecular partition functions, as in ref. 395. The most attractive features of the RxMC method are its versatility and the fact that the information it requires (i.e. the molecular partition functions) is readily available for most reactions. Since its inception, RxMC has been applied to a variety of reactive systems, including organic and inorganic reactions in gases, solutions, plasmas, and in nanoporous 
materials ${ }^{396,398-410}$. So far RxMC seems to be the best choice for the estimation of chemical equilibrium constants in non-ideal systems.

\subsubsection{Transition State Theory and the Reactive Flux Method Me,13-15,411-413 $^{2,4}$}

One of the early attempts to calculate reaction rates, and arguably the most successful, is the transition state theory (TST) of Eyring ${ }^{414,415}$, sometimes also called activated complex theory or absolute rate theory ${ }^{14}$. Since its inception, the method has been extended and reformulated in several different ways ${ }^{15,412}$. The original formulation of TST is in thermodynamic terms. TST states that, for a reactive mixture in thermal equilibrium, the reaction rate may be obtained from the equilibrium constant for the conversion of reactant molecules to a transition state molecule $\mathrm{e}^{3,13-15}$ :

$$
k_{r}^{0}=\frac{k T}{h} K_{e q}^{\ddagger}=\frac{k T}{h} \exp \left(-\Delta G^{\ddagger} / k T\right)=\frac{k T}{h} \frac{a^{\ddagger}}{\prod_{\text {reac }} a_{i}^{-v_{i}}}
$$

where the product runs over the reactants, $\Delta G^{*}$ is the free energy of activation, i.e. the change in the free energy of the system when reactant molecules are replaced by a transition state molecule, and $k_{r}^{0}$ is the standard-state reaction rate constant. TST thus reduces the dynamical problem of finding a reaction rate to an equilibrium problem that, as discussed in the previous sections, is much easier to solve. If, for example, the intermolecular potential effects are neglected, one may use (2.5.2) and (2.5.6) to write the transition state equilibrium constant in term of molecular partition functions. In this case there is, however, an important difference. Since a transition state species corresponds to a first-order saddle point in the potential energy surface, it has one less vibrational mode than a stable molecule. The transition state does not occur at a minimum, but at a maximum free energy in the direction of the reaction coordinate. Hence, when calculating 
the vibrational partition function, there will be one vibrational mode missing. The term $k T / h$ appearing in (2.5.6) can be interpreted as the contribution to the partition function corresponding to the non-vibrational motion along the reaction coordinate, i.e. as the term replacing the missing vibrational term ${ }^{13}$.

It is also possible to formulate TST in dynamical terms ${ }^{416,417}$. This formulation requires introducing the concept of a dividing surface, which separates the "reactants" and "products" regions of configurational space. In TST, the dividing surface can be defined as the plane normal to the reaction path and containing the transition state. Then the reaction rate can be defined as the net rate at which the system crosses the dividing surface in the direction of the products. For simplicity, let us consider an isomerization reaction $\mathrm{A} \rightarrow \mathrm{B}$. It can be shown that its reaction rate is given by the stationary-state value of the timecorrelation function $k_{r}(t)$ defined by ${ }^{417}$ :

$$
k_{r}(t)=\frac{\left\langle\dot{\xi}(0) \theta\left[\xi(t)-\xi^{\ddagger}\right] \delta\left[\xi^{\ddagger}-\xi(0)\right]\right\rangle}{\left\langle C_{A}\right\rangle}
$$

In (2.5.7), $C_{A}$ is the concentration of the reactant species $A,\left\langle C_{A}\right\rangle=\left\langle\theta\left[\xi(0)-\xi^{\ddagger}\right]\right\rangle, \xi(t)$ is the reaction coordinate as a function of time, $\xi^{\ddagger}$ is the value of the reaction coordinate at the dividing surface, $\theta$ is the Heaviside step function, $\delta$ the Dirac delta function, and the brackets indicate an ensemble average over equilibrium initial conditions ${ }^{418}$. Note that the term $\delta\left[\xi^{\ddagger}-\xi(0)\right]$ constrains the system to be initially at the dividing surface. The reaction rate is then:

$$
k_{r}=\lim _{t \rightarrow \infty} k_{r}(t)=\lim _{t \rightarrow \infty} \frac{\left\langle\dot{\xi}(0) \theta\left[\xi(t)-\xi^{\ddagger}\right] \delta\left[\xi^{\dagger}-\xi(0)\right]\right\rangle}{\left\langle C_{A}\right\rangle}
$$


Equation (2.5.8) is often called the Bennett ${ }^{419}$-Chandler ${ }^{420-422}$ expression for the rate constant. In taking the limit, the time is supposed to be large compared to the characteristic time for the molecular motion, but small compared to the characteristic time for the reaction. Thus in the derivation of (2.5.8) it is implicitly assumed that the reaction is a rare event compared to molecular relaxation. This will be true as long as the free energy barrier for the reaction is large compared to $k T$.

It is possible to recover the transition state theory constant by taking the limit as $t \rightarrow 0^{+}$of $k_{r}(t)$ :

$$
k_{r, T S T}=\lim _{t \rightarrow 0^{+}} \frac{\left\langle\dot{\xi}(0) \theta\left[\xi(t)-\xi^{\ddagger}\right] \delta\left[\xi^{\ddagger}-\xi(0)\right]\right\rangle}{\left\langle C_{A}\right\rangle}=\frac{\left\langle\dot{\xi}(0) \theta[\dot{\xi}(0)] \delta\left[\xi^{\ddagger}-\xi(0)\right]\right\rangle}{\left\langle C_{A}\right\rangle}
$$

Here we have used the fact that $\xi(t)$ will be larger than $\xi^{*}$ as $t \rightarrow 0^{+}$only if the derivative $\dot{\xi}(0)$ is positive, because only configurations starting at $\xi^{\ddagger}$ contribute to the average. It may not be directly evident that (2.5.9) is equivalent to (2.5.6); for a derivation of this see, e.g. refs. 423,424. Comparing equation (2.5.9) with (2.5.8) gives a clearer interpretation of the TST approximation: the two expressions will be equal only if, after initially crossing the dividing surface, the system does not return to the "reactants side", i.e. no recrossings of the dividing surface occur. This gives us another, more precise way of stating the quasiequilibrium assumption, which is to say that all trajectories crossing the dividing surface in the direction of the products and which do not recross it are in equilibrium with the reactants ${ }^{412}$.

We already mentioned that, for (2.5.8) to be valid, it is necessary that the reaction barrier is high compared to $k T$. From the above reasoning, we see that TST will be valid when, after crossing the dividing surface, the reactive complex is able to dissipate its energy fast 
enough so that it cannot go back again. If this is not true, some recrossings of the dividing surface can occur and the real reaction rate will be lower than the TST rate. In order to account for this, one may define a transmission coefficient $\kappa \leq 1$ that corrects the TST rate constant due to the recrossings:

$$
k_{r}=\kappa k_{r, T S T}, \kappa \leq 1
$$

Unlike the TST constant, the transmission coefficient is a dynamical quantity and cannot be obtained from an equilibrium simulation such as RxMC. The fact that $\kappa \leq 1$ implies that the TST constant is an upper bound for the true reaction rate.

There is one important remark to make about equation (2.5.10). This expression is correct even when the dividing surface is not exactly at the transition state. What is used in its derivation $^{417}$ is the separation of timescales between the reaction process and the molecular motion, and not the fact that the dividing surface contains the transition state. Now, since the $k_{r, T S T}$ in (2.5.10) is always an upper bound for the reaction rate regardless of how the dividing surface is chosen, this suggests a way to improve the TST estimate: do a TST calculation for different dividing surfaces and choose the lowest one. This is the approach used in variational transition state theory $(\mathrm{VTST})^{425}$.

One may ask why is it necessary to search for a dividing surface that does not contain the transition state. After all, it sounds reasonable to assume that the surface least likely to be recrossed is the one containing the transition state, and hence the TST reaction rate should be the lowest for that case. Nevertheless, it is important to take into account the fact that the PES does not include finite-temperature effects. Also the reactive system may be in a medium that imposes a force field on the atoms that was not taken into account during the $a b$ initio calculations (e.g. an attractive surface, a solvent). Hence the true saddle point in the real free energy surface (FES) may be quite different than the one in the PES. By using 
a VTST approach this can be accounted for. As an example, for a reactive mixture of ideal gases the TST rate constant can be written, using (2.5.6) and (2.5.2), as:

$$
k_{r, T S T}=\frac{k T}{h} K_{e q}^{\ddagger}=\frac{k T}{h} \frac{q^{\ddagger} / \mathrm{V}}{\prod_{\text {reac }}\left(q_{i} / V\right)^{-v_{i}}}
$$

In order to minimize this rate, it is necessary to choose the dividing surface such that the molecular partition function of the transition state, $q^{\ddagger}$, will be minimal. Since this function contains the contributions from vibrational, rotational, etc. modes, it accounts for the finite-temperature effects not considered in the PES.

What we have given so far is a description of TST in purely classical terms. Due to the success of TST as both a theoretical and computational tool for understanding rate processes, there has been considerable effort to cast TST in quantum mechanical terms. This is a difficult problem, though, because of TST's natural formulation in terms of classical dynamical trajectories. In quantum TST (QTST), the objective is to obtain a quantum mechanical expression for the time-correlation function. Among the quantumbased versions of QTST are the short-time QTST method of Miller et al. ${ }^{426-428}$ and its variational version $^{429}$, the path integral QTST methods ${ }^{430-432}$, including the reversible action-space work QTST (RAW-QTST) method $^{433,434}$, and several others ${ }^{435-441}$. A discussion of QTST and related methods is beyond the scope of this work; reviews can be found in refs. 411,412,442,443. One important contribution that is missing in classical TST, besides the transmission coefficient, is the quantum-tunneling correction. For some systems (e.g. reactions involving light molecules, low temperatures), it is possible for the reactants to tunnel through the activation barrier, and hence the reaction rate will be higher than that obtained by TST. A quantum mechanical treatment is necessary to obtain this correction. 
Equations (2.5.7)-(2.5.10) suggest a natural way to correct a TST rate constant: obtain the transmission coefficient. In the reactive flux method, or Bennet-Chandler method, the reaction rate is obtained by using (2.5.7) directly, which is equivalent to finding the TST rate and the transmission coefficient. For this it is convenient to rewrite $(2.5 .7)$ as ${ }^{11}$ :

$$
k_{f}(t)=\frac{\left\langle\dot{\xi}(0) \theta\left[\xi(t)-\xi^{\ddagger}\right] \delta\left[\xi^{*}-\xi(0)\right]\right\rangle}{\left\langle\delta\left[\xi^{\ddagger}-\xi(0)\right]\right\rangle} \frac{\left\langle\delta\left[\xi^{*}-\xi\right]\right\rangle}{\left\langle\theta\left[\xi^{*}-\xi\right]\right\rangle}
$$

The first term in this equation is the conditional average of the product $\dot{\xi}(0) \theta\left[\xi(t)-\xi^{*}\right]$, given that the system is initially at the dividing surface, i.e. $\xi(0)=\xi^{\ddagger}$. This term is a dynamical quantity, like the transmission coefficient. The second term is a ratio of the probability density of finding the system at the dividing surface to the probability of finding the system in the reactant side of the barrier. This term, like the TST constant, is an equilibrium quantity and can be obtained from a thermodynamic integration. In the next section we will discuss a combined approach for finding the reaction rate from $(2.5 .12)$ using constrained dynamics.

One important comment about the methods discussed in this and the following sections is that studying chemical reactions is not their only application. The kinetic rate for any process that involves crossing an energy barrier can be obtained within the formalism. This includes, for example, nucleation ${ }^{444}$ and diffusion in solid surfaces ${ }^{445,446}$. Finally, an improved reactive flux method allowing the estimation of rate constants with a lower computational effort has been recently proposed by Drozdov and Tucker ${ }^{447}$. 


\subsubsection{Constrained Reaction Dynamics: “Blue Moon” Method.}

As discussed in the previous section, it is possible to find good estimates of kinetic rate constants by separately evaluating the two factors in (2.5.12). The first term is a conditional average over trajectories starting at the dividing surface, and it can be evaluated from molecular dynamics (MD) simulations with the system initially at the top of the barrier. In this case, it is necessary to correct the statistics using bias factors to get the right averages. The second factor in (2.5.12) can be obtained also from constrained dynamics, and hence it is possible to use a unified two-step MD approach to calculate the reaction rate. This is the so-called "Blue Moon" ${ }^{448}$ approach for finding reaction rates ${ }^{11,449-}$ 453.

In the Blue Moon method, a constrained MD run with the reaction coordinate held fixed at the constant value $\xi=\xi^{\ddagger}$ is used to generate configurations at the top of the barrier and also to estimate the second term in (2.5.12) using the constraint force. Then these configurations can be used in a second set of MD runs where the constraint is relaxed and the atoms are assigned initial velocities from a Maxwell-Boltzmann distribution for the given temperature. These runs are used to accumulate statistics on the number of pathways with $\dot{\xi}(0)>0$ that reach the "products" region of space, thus allowing the estimation of the first term in (2.5.12).

In order to derive expressions for the proper averages when the Blue Moon technique is used, let us start from the canonical ensemble probability distribution for states with a prespecified value of the reaction coordinate, $\xi=\xi^{\ddagger 449}$ :

$$
P_{\mathrm{I}}\left(\mathbf{r}^{N}, \mathbf{p}^{N}\right) \delta\left(\xi-\xi^{\ddagger}\right) d \mathbf{r}^{N} d \mathbf{p}^{N}=\frac{1}{Q_{\mathrm{I}}} \exp \left(-\frac{H}{k T}\right) \delta\left(\xi-\xi^{\ddagger}\right) d \mathbf{r}^{N} d \mathbf{p}^{N}
$$


We will call this ensemble I. In this equation, $H$ is the system Hamiltonian, $Q_{I}$ is the partition function and $\delta$ is the Dirac delta function. Note that in ensemble I the reaction coordinate is restricted to the value $\xi=\xi^{\ddagger}$, but is not constrained, i.e. its time derivative $\dot{\xi}$ is not restricted to be zero. The phase-space points generated in this ensemble could be used as starting points for an MD simulation, and would generate reacting trajectories frequently. The probability distribution (2.5.13) can be factored as:

$$
P_{\mathrm{I}}\left(\mathbf{r}^{N}, \mathbf{p}^{N}\right) \delta\left(\xi-\xi^{\ddagger}\right) d \mathbf{r}^{N} d \mathbf{p}^{N}=P_{c, \mathrm{I}}\left(\mathbf{r}^{N}\right) P_{m, \mathrm{I}}\left(\mathbf{p}^{N} \mid \mathbf{r}^{N}\right) \delta\left(\xi-\xi^{\ddagger}\right) d \mathbf{r}^{N} d \mathbf{p}^{N}
$$

Where $P_{c, I}\left(\mathbf{r}^{N}\right)$ is the configurational probability density and $P_{m, I}\left(\mathbf{p}^{N} \mid \mathbf{r}^{N}\right)$ is the conditional probability density of the momenta given the configuration. Only the latter will be of interest to us. It can be expressed as:

$$
P_{m, I}\left(\mathbf{p}^{N} \mid \mathbf{r}^{N}\right) d \mathbf{p}^{N}=\exp \left(-\frac{K}{k T}\right) d \mathbf{p}^{N}
$$

where $K$ is the kinetic energy:

$$
K=\sum_{j=1}^{N} \frac{\mathbf{p}_{j} \cdot \mathbf{p}_{j}}{2 m_{j}}
$$

The probability density (2.5.15) is a product of Maxwell distributions for individual atoms, and it is fairly straightforward to sample from it. Now we will introduce an ensemble II where the reaction coordinate is not only restricted to the value $\xi=\xi^{\ddagger}$, but also constrained to it (i.e. where $\dot{\xi}=0$ ). 
The configurational part of the probability distribution for ensemble II will be:

$$
P_{c, \mathrm{II}}\left(\mathbf{r}^{N}\right) d \mathbf{r}^{N}=\frac{1}{Q_{\mathrm{II}}}|\mathbf{Z}|^{1 / 2} \exp \left(-\frac{\mathcal{U}}{k T}\right) \delta\left(\xi-\xi^{\sharp}\right) d \mathbf{r}^{N}
$$

where $\mathcal{U}=\mathcal{U}\left(\mathbf{r}^{N}\right)$ is the potential energy and $Q_{\mathrm{II}}$ is the partition function for this ensemble. The term $|\mathbf{Z}|^{1 / 2}$ is a bias factor arising from the constraint imposed on the reaction coordinate. For the case being discussed (where only the reaction coordinate is constrained), it can be obtained from:

$$
|\mathbf{Z}|=\sum_{j=1}^{N} \frac{1}{m_{j}}\left(\frac{\partial \xi}{\partial \mathbf{r}_{j}}\right)^{2}
$$

The derivation for the more general case when other constraints are imposed on the system is discussed in refs. 449,451 .

At this stage, we recognize that both ensembles I and II are difficult to sample in a MD run. In ensemble I, since there is no restriction on the derivative of the reaction coordinate, it is fairly easy to sample from the momentum distribution, but it is difficult to generate the configurations. We would need to keep $\xi=\xi^{\ddagger}$ as time goes on, but without having $\dot{\xi}=0(!)$.

In ensemble II, on the other hand, we can easily generate configurations because $\dot{\xi}=0$. The problem, however, is that we cannot use the states from ensemble II to get statistics on how the system crosses the energy barrier because of the constraint. This reasoning suggests defining a new ensemble, the blue moon ensemble $e^{449}$, with the configurational 
probability density of ensemble II and the conditional momenta probability density of ensemble I, i.e.

$$
\begin{aligned}
P_{\mathrm{BM}}\left(\mathbf{r}^{N}, \mathbf{p}^{N}\right) d \mathbf{r}^{N} d \mathbf{p}^{N} & =P_{c, \mathrm{II}}\left(\mathbf{r}^{N}\right) P_{m, \mathrm{I}}\left(\mathbf{p}^{N} \mid \mathbf{r}^{N}\right) d \mathbf{r}^{N} d \mathbf{p}^{N} \\
& =\frac{1}{Q_{\mathrm{II}}}|\mathbf{Z}|^{1 / 2} \exp \left(-\frac{H}{k T}\right) \delta\left(\xi-\xi^{\ddagger}\right) d \mathbf{r}^{N} d \mathbf{p}^{N}
\end{aligned}
$$

We now have an ensemble that is easier to sample. We need to generate configurations with a constrained value of the reaction coordinate, and velocities from a Maxwell distribution. This procedure will generate a set of configurations that, if used as starting points for a MD, will produce barrier crossings frequently. The true dynamic trajectories, however, are the ones of ensemble I, where $\dot{\xi}$ is not restricted, and therefore we need to include a bias factor when averaging over the blue moon ensemble.

If we want to obtain the average of the observable $A\left(\mathbf{r}^{N}, \mathbf{p}^{N}\right)$ over ensemble I, we need to calculate:

$$
\langle A\rangle=\frac{\left\langle A\left(\mathbf{r}^{N}, \mathbf{p}^{N}\right) \delta\left(\xi-\xi^{\ddagger}\right)\right\rangle_{I}}{\left\langle\delta\left(\xi-\xi^{\ddagger}\right)\right\rangle_{I}}
$$

Comparing the probability distribution of ensemble I, equation (2.5.13), with the probability distribution of the blue moon ensemble, equation (2.5.19), we see that this is equivalent to:

$$
\langle A\rangle=\frac{\left\langle|\mathbf{Z}|^{-1 / 2} A\left(\mathbf{r}^{N}, \mathbf{p}^{N}\right)\right\rangle_{B M}}{\left\langle|\mathbf{Z}|^{-1 / 2}\right\rangle_{B M}}
$$


Thus the factor $|\mathbf{Z}|^{-1 / 2}$ is what we need to correct the bias caused by the constraint $\dot{\xi}=0$ in the configurational distribution. The averages in (2.5.21) are now taken in the blue moon ensemble.

The derivation above pertains to the case where only the reaction coordinate is being constrained. Sometimes it is convenient to include additional constraints in the simulation, for example fixed bond lengths in a molecule. For the general case when $L$ additional holonomic ${ }^{454}$ constraints of the form $\sigma_{i}\left(\mathbf{r}^{N}\right)=0, i=1, \cdots, L$ are included, equation (2.5.21) has to be modified. The corresponding equations can be found in refs. 449,451 .

We may now turn to the problem of calculating reaction constants in the blue moon ensemble. For this we need to rewrite the averages in (2.5.12) as averages in the blue moon ensemble. For the first factor in (2.5.12), we can directly use the result (2.5.21):

$$
\frac{\left\langle\dot{\xi}(0) \theta\left[\xi(t)-\xi^{*}\right] \delta\left[\xi^{*}-\xi(0)\right]\right\rangle}{\left\langle\delta\left[\xi^{*}-\xi(t)\right]\right\rangle}=\frac{\left\langle|\mathbf{Z}|^{-1 / 2} \dot{\xi}(0) \theta\left[\xi(t)-\xi^{*}\right]\right\rangle_{B M}}{\left\langle|\mathbf{Z}|^{-1 / 2}\right\rangle_{B M}}
$$

The second factor in (2.5.12), as we mentioned earlier, is the probability density of finding the system at the top of the barrier, divided by the probability that the system is on the "reactants" side of the dividing surface. This can also be obtained from the constrained MD in the blue moon simulation. We will define the probability of finding the system at any value of the reaction coordinate $\xi=\xi^{\prime}$ (not necessarily at the top of the energy barrier) as:

$$
P\left(\xi^{\prime}\right)=\frac{\int d \mathbf{r}^{N} \exp (-\mathcal{U} / k T) \delta\left(\xi^{\prime}-\xi\right)}{\int d \mathbf{r}^{N} \exp (-\mathcal{U} / k T) \theta\left(\xi^{\star}-\xi\right)}
$$


Differentiating the logarithm of $P\left(\xi^{\prime}\right)$ with respect to $\xi^{\prime}$, we obtain:

$$
\frac{\partial \ln P\left(\xi^{\prime}\right)}{\partial \xi^{\prime}}=\frac{\int d \mathbf{r}^{N} \exp (-\mathcal{U} / k T) \partial \delta\left(\xi^{\prime}-\xi\right) / \partial \xi^{\prime}}{\int d \mathbf{r}^{N} \exp (-\mathcal{U} / k T) \delta\left(\xi^{\sharp}-\xi\right)}
$$

The negative of this quantity (times $k T$ ) is the mean force associated with the reaction coordinate constraint. In order to remove the derivative of the Dirac delta in (2.5.24), we need to integrate by parts. However, the dependence of $\xi$ on the configuration in (2.5.24) is implicit. It is convenient to define a new set of generalized coordinates $\left(\mathbf{q}^{N-1}, \xi\right)$ where $\xi$ appears explicitly. In terms of these coordinates, (2.5.24) is:

$$
\frac{\partial \ln P\left(\xi^{\prime}\right)}{\partial \xi^{\prime}}=\frac{\int d \mathbf{q}^{N-1} d \xi|\mathbf{J}| \exp (-\mathcal{U} / k T) \partial \delta\left(\xi^{\prime}-\xi\right) / \partial \xi^{\prime}}{\int d \mathbf{r}^{N} \exp (-\mathcal{U} / k T) \delta\left(\xi^{\ddagger}-\xi\right)}
$$

Where $|\mathbf{J}|$ is the Jacobian of the transformation $\mathbf{r}^{N} \rightarrow\left(\mathbf{q}^{N-1}, \xi\right)$. The exact form of this transformation will depend on the functional form of the reaction coordinate.

Integrating the numerator of (2.5.25) by parts, we find:

$$
\begin{aligned}
\frac{\partial \ln P\left(\xi^{\prime}\right)}{\partial \xi^{\prime}} & =\frac{\int d \mathbf{q}^{N-1} d \xi \delta\left(\xi^{\prime}-\xi\right) \partial[|\mathbf{J}| \exp (-\mathcal{U} / k T)] / \partial \xi}{\int d \mathbf{r}^{N} \exp (-\mathcal{U} / k T) \delta\left(\xi^{\ddagger}-\xi\right)}= \\
& =\frac{\int d \mathbf{q}^{N-1} d \xi \delta\left(\xi^{\prime}-\xi\right) \exp (-\mathcal{U} / k T) \partial\{\ln [|\mathbf{J}|-\mathcal{U} / k T]\} / \partial \xi}{\int d \mathbf{r}^{N} \exp (-\mathcal{U} / k T) \delta\left(\xi^{\ddagger}-\xi\right)}
\end{aligned}
$$


The latter expression can be expressed in terms of averages in the blue moon ensemble by using (2.5.21):

$$
\frac{\partial \ln P\left(\xi^{\prime}\right)}{\partial \xi^{\prime}}=\frac{\left\langle|\mathbf{Z}|^{-1 / 2} \partial[|\mathbf{J}| \exp (-\mathcal{U} / k T)] / \partial \xi\right\rangle_{B M}}{\left\langle|\mathbf{Z}|^{-1 / 2}\right\rangle_{B M}}
$$

If we now want the second factor in (2.5.12), we need to integrate this expression from $\xi^{\prime}=0 \quad$ (the reactant side) to $\xi^{\prime}=\xi^{\ddagger}$. The expression will therefore be:

$$
\frac{\left\langle\delta\left[\xi^{\ddagger}-\xi(t)\right]\right\rangle}{\left\langle\theta\left[\xi^{\ddagger}-\xi(t)\right]\right\rangle}=\int_{0}^{\xi^{\ddagger}} d \xi \frac{\left\langle|\mathbf{Z}|^{-1 / 2} \partial[|\mathbf{J}| \exp (-\mathcal{U} / k T)] / \partial \xi\right\rangle_{B M}}{\left\langle|\mathbf{Z}|^{-1 / 2}\right\rangle_{B M}}
$$

This expression has to be calculated numerically from runs in the blue moon ensemble. It is possible $e^{449,452}$ to relate the integral on the right-hand side to the Lagrangian multiplier associated with the constraint $\xi=\xi^{*}$, which is obtained during the constrained MD run using an algorithm such as $\mathrm{SHAKE}^{328}$. Therefore the second term in (2.5.12) can be obtained from quantities that are already calculated during the constrained MD run.

Summing up all the information we have, we may write the procedure for obtaining rate constants from blue moon MD:

1. Specify a value for the reaction coordinate, $\xi^{\ddagger}$, at the dividing surface.

2. Generate phase space trajectories where the reaction coordinate is constrained to $\xi=\xi^{\ddagger}$. These can be obtained from a constrained MD algorithm such as SHAKE.

3. Choose a set of configurations generated in step 2. For each of these:

3.1 Assign velocities to the particles from a Maxwell-Boltzmann distribution. 
3.2 Using the configuration from 2 and the velocities from 3.1, run an MD simulation for a time long enough for the system to reach the reactants or products basin.

3.3 Record the final state of the system. Accumulate the number of configurations with $\dot{\xi}>0$ that reach the products basin.

4. Using the results from step (3), compute the first term in (2.5.12) using (2.5.22).

5. Obtain the second term in (2.5.12) by numerically integrating the mean constraint force, i.e. using equation (2.5.28).

6. Finally, use (2.5.12) to obtain the reaction rate.

A schematic of this is shown in figure 2.5.1. An advantage of the Blue Moon MD approach is that it can in principle handle systems as large as a standard MD simulation ${ }^{455}$. The time interval spanned in each MD run in step 3 may also be chosen to be the same as in a standard MD simulation ${ }^{455}$, but the "real-time" interval spanned in the blue moon ensemble is much longer. The reason for this is that Blue Moon MD "jumps" from one reaction event to another, not simulating in detail over the long time intervals between two events $^{449}$.

One disadvantage of blue moon $\mathrm{MD}$ is that the reaction must be simple enough to have a clearly defined energy barrier and reaction coordinate. Recently, however, the method has been extended to handle more complex systems ${ }^{451}$. Another problem is that it is necessary to impose a definition of the reaction coordinate beforehand. If this reaction coordinate is too far from the intrinsic reaction coordinate, the results will not be good. It is important to verify that the reaction coordinate chosen is a good one. A way to do this that is amenable to the Blue Moon MD method is to repeat the calculation of mean forces with a reversed reaction path. If a large hysteresis loop appears, the reaction coordinate is not adequate $^{456,457}$ and it is necessary to choose another one. 


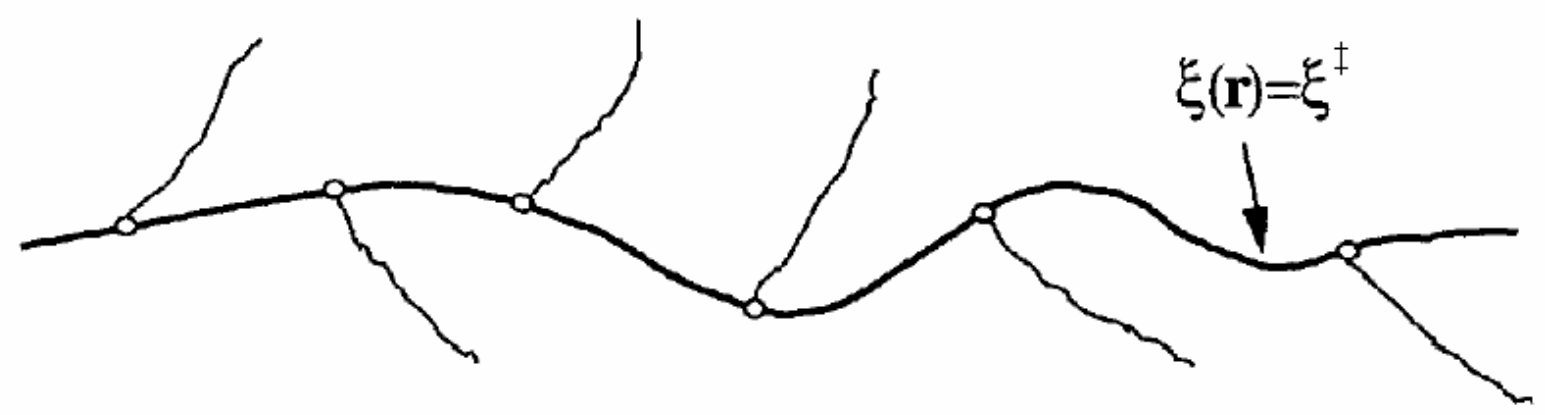

Figure 2.5.1. Sampling in the Blue Moon method. The thick line represents the phase space trajectory with the reaction coordinate constrained, which is used to obtain the second factor in (2.5.12). The thin lines are unconstrained trajectories starting at configurations from the constrained simulation; these are used to obtain the first factor in (2.5.12). From ref. 450 .

The most recent applications of the blue moon MD methodology are in combination with $a b$ initio molecular dynamics, due to the ability of the latter to generate dynamic trajectories while taking the electronic degrees of freedom into account explicitly. Some of these applications include:

- A study of the intramolecular solvation effects on the reaction of a chloride anion with several cyano-chloroalkanes ${ }^{458}$. In this work the blue moon methodology in conjunction with CPMD was used to find the thermal effects on the PES for the reactions considered.

- An $a b$ initio MD study of the reaction of the fluoride ion with chloromethane ${ }^{459}$ and chloroethane $^{460}$. In this work, as in the previous one, the blue moon MD methodology is used to incorporate the thermal effects on the PES in combination with the CPMD method. 
- A calculation of the $\mathrm{p} K_{a}$ for the axial and equatorial hydroxyl groups in aqueous $\mathrm{P}(\mathrm{OH})_{5}$ using constrained $a b$ initio molecular dynamics ${ }^{461}$. The calculated values in this work show good agreement with experimental results for a related system (aqueous tetracyclohexyloxyhydroxyphosphorane).

\subsubsection{Transition Path Sampling.}

As mentioned in the previous sections, when a system has a PES that depends on a small number of degrees of freedom, it is not too difficult to find the relevant transition state(s) and reaction coordinate(s). For more complex systems with high-dimensional PES, however, there are usually many local minima and saddle points and it becomes much harder to determine the relevant reaction paths. This is illustrated in figure 2.5.2. The

Transition Path Sampling (TPS) method ${ }^{462-468}$ offers a way to determine reaction rates for such systems.

There is no single way to deal with the problem of the separation of timescales between reactions and molecular relaxation. In the Blue Moon MD method, for example, this problem is overcome by creating an ensemble of initial states close to the top of the energy barrier, running simulations starting from these states, and then obtaining reaction rates from ensemble averages over the results of these runs. Nevertheless, if the energy landscape is complicated, one does not know where the top of the barrier is to begin with, and it is even possible that several different paths contribute to the reaction rate. In TPS, as in Blue Moon MD, we generate an ensemble of dynamic trajectories and find the reaction rate from averages on this ensemble. The trajectories, however, are not restricted to start from the top of any barrier. 

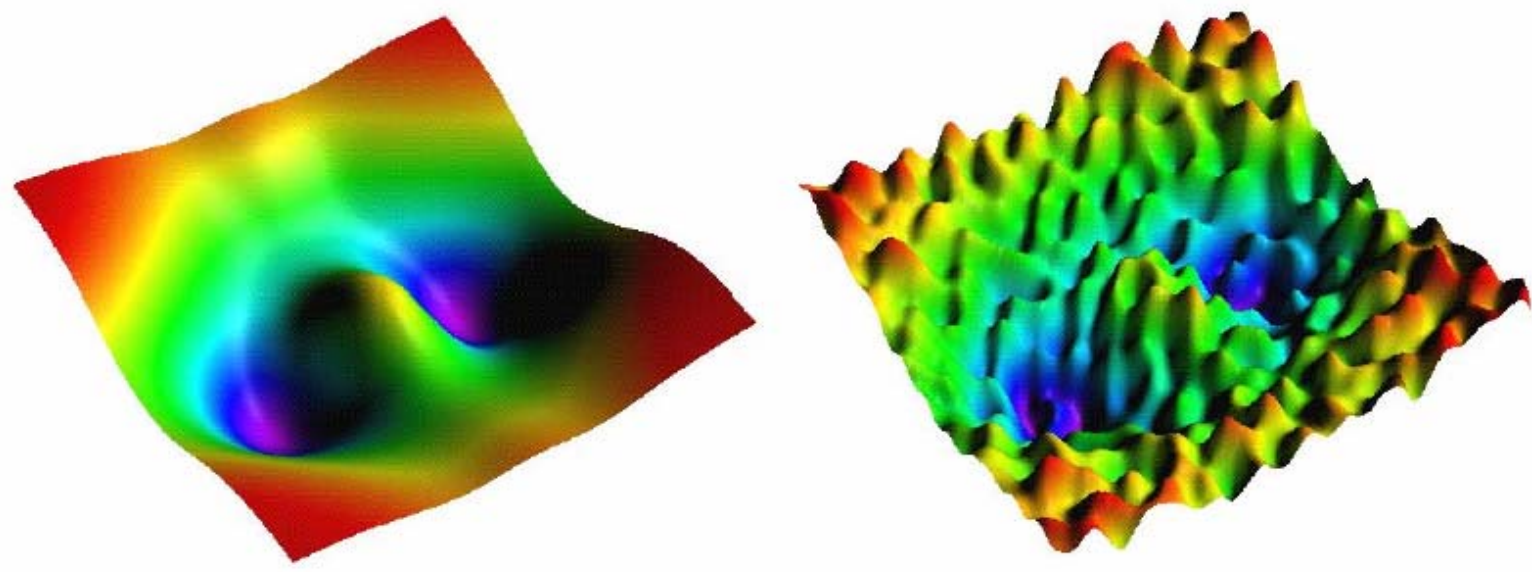

Figure 2.5.2 For simple reactions that do not involve many degrees of freedom it is usually easy to determine the reaction mechanism by locating the energy minima and saddle points (left). In complex reactions where many degrees of freedom are involved there may be many saddle-points and local minima, and hence many possible reaction pathways (right). From ref. 468.

The basic idea behind TPS is to construct reactive trajectories that go from the reactants basin (A) to the products basin (B) in the potential energy surface, and then sample over these trajectories ${ }^{462}$. The transition path ensemble is defined as the collection of all trajectories starting at $\mathrm{A}$ and ending at $\mathrm{B}$ after a given time $t_{F}$. The trajectories are defined as a set of points in phase space $\{\Gamma(0), \Gamma(\delta t), \Gamma(2 \delta t), \cdots, \Gamma(M \delta t)\}$, where $\delta t$ is the timestep and $M \delta t=t_{F}$. These trajectories can be chosen to be Verlet ${ }^{11,12,317,318}$ trajectories, although other definitions are possible ${ }^{468}$. It is clear from this definition that, in order to run a TPS simulation, we need to identify beforehand the reactant and products basins, and choose a time $t_{F}$. It is also necessary to find a reactive trajectory to start the simulation. These points are discussed below. 
In order to find a reactive trajectory, one possibility is to run a MD simulation at conditions for which the reaction is no longer a rare event, such as a higher temperature, and then anneal the system to obtain a reactive trajectory at a lower temperature. This procedure, however, is very laborious. An alternative is to use an action-based method, i.e. find a dynamic path connecting a reactant and a product state that corresponds to a stationary point of the classical action. A procedure for doing this in the context of CPMD has been proposed in ref. 469. Other alternatives for finding an initial trajectory are discussed in ref. 468.

Since the molecular equations of motion are non-linear, the trajectories generated from them are chaotic and hence sensitively dependent on the initial conditions. Therefore, a small change in a position or momentum in one time slice of a given trajectory will cause it to diverge from the original one after some time. Once this happens, the trajectory becomes uncorrelated with the original one and depends solely on the potential energy surface. The total path time $t_{F}$ has, thus, to be larger than the time required for the trajectory to diverge after a small change to get a correct sampling of the true dynamics. The simplest way to do this is to take the initial reactive path, change the momentum of one time-slice in the smallest possible value representable on the computer, and integrate the dynamic equations forward and backwards in time. The time after which the trajectories have diverged from the original one, multiplied by some factor (usually around 10), is taken as $t_{F}$.

Defining the basins of reactants and products is one of the crucial points in TPS. If the reactants and/or products basin is defined as a too narrow region of phase space, it is likely that many reactive trajectories are not sampled, yielding a wrong value of the reaction constant. On the other hand, if they are too large, there is a chance that non-reactive trajectories are counted as reactive. In most cases, defining the basins of attraction is a trial-and-error procedure, as described in ref. 468. 
Once the parameters of the simulation are set and an initial reacting trajectory is known, the simulation proceeds by generating new trajectories and checking if they are still reactive, i.e. if they still connect regions $\mathrm{A}$ and $\mathrm{B}$. The new trajectories may be generated in several different ways, the two most common being shooting and shifting moves. Shooting moves are the simplest possible. In this case a time slice from the original trajectory is selected at random and the momenta of the particles are randomly changed by a small amount $\delta p$. Then the equations of motion are integrated either forward in time to $t_{F}$, or backwards in time to $t=0$. If the new trajectory still connects regions $\mathrm{A}$ and $\mathrm{B}$, it is accepted. In a shifting move, an additional number of time slices $l$ is added to either the beginning of the trajectory (by integrating backwards in time from $t=0$ to $t=-l \delta t$ ) or its end (by integrating forward in time from $t=t_{F}$ to $t=t_{F}+l \delta t$ ). When the shift is done at the beginning of the trajectory, the last $l$ slices are discarded and when it is done at the end, the first $l$ slices are discarded. This is done to keep the total number of time-steps fixed. If the new trajectory still connects regions A and B, it is accepted.

Once the set of trajectories is generated, the reaction rate can be obtained through expressions similar to the ones used in the reactive flux method.

First, the $\theta(\mathbf{x})$ functions are defined as:

$$
\theta_{A, B}(\mathbf{x})= \begin{cases}1 & \text { for } \mathbf{x} \in A, B \\ 0 & \text { for } \mathbf{x} \notin A, B\end{cases}
$$

These are the equivalent of the $\theta$ function in Blue Moon MD. The $\theta_{A}$ function is one for configurations in the reactants basin, and zero outside, and the $\theta_{B}$ function is one for configurations in the products basin, and zero outside. 
In terms of these functions, the forward reaction rate will be the stationary value of:

$$
k_{f}(t)=\frac{\left\langle\theta_{A}(\mathbf{x}(0)) \dot{\theta}_{B}(\mathbf{x}(t))\right\rangle}{\left\langle\theta_{A}(\mathbf{x}(0))\right\rangle}
$$

This is analogous to equation (2.5.7) for the reactive flux method. It expresses that the reaction rate is proportional to the time derivative of the probability that the system is in region $\mathrm{B}$ at time $t$, provided that it started from region A at time 0. Equation (2.5.30) is often rewritten as:

$$
k_{F}(\tau \delta t)=\frac{\left\langle\theta_{A}\left(\mathbf{x}_{0}\right) \dot{\theta}_{B}\left(\mathbf{x}_{\tau}\right)\right\rangle}{\left\langle\theta_{A}\left(\mathbf{x}_{0}\right) \theta_{B}\left(\mathbf{x}_{M}\right)\right\rangle} \frac{\left\langle\theta_{A}\left(\mathbf{x}_{0}\right) \theta_{B}\left(\mathbf{x}_{M}\right)\right\rangle}{\left\langle\theta_{A}\left(\mathbf{x}_{0}\right)\right\rangle}=\left\langle\dot{\theta}_{B}\left(\mathbf{x}_{\tau}\right)\right\rangle_{A B} \frac{\left\langle\theta_{A}\left(\mathbf{x}_{0}\right) \theta_{B}\left(\mathbf{x}_{M}\right)\right\rangle}{\left\langle\theta_{A}\left(\mathbf{x}_{0}\right)\right\rangle}
$$

Where $\tau \equiv t / \delta t$ is a dimensionless time. This equation is also analogous to (2.5.12). The first factor can be obtained directly from the TPS simulation, but the second factor requires additional work. The first term is the time derivative of the probability that, in a trajectory connecting $\mathrm{A}$ and $\mathrm{B}$, region $\mathrm{B}$ is reached after $\tau$ time steps. For $k_{F}$ to attain a stationary value it is necessary that this first factor also attain a stationary value within the time frame studied. This can be used as a test to check if the transition path is long enough: if the stationary value is not frequently attained, it is necessary to use a larger number of time slices, $M$.

The second factor in (2.5.31) is the probability that a system starting in region A reaches region $\mathrm{B}$ in $M$ time steps. This cannot be obtained directly from TPS, because all trajectories in the TPS ensemble are assumed to start at A and end in B. It cannot be obtained either from a standard simulation because the reaction is a rare event (otherwise we would have used a standard simulation to begin with). One way around this problem is 
very similar to the thermodynamic integration used to find the second factor of $(2.5 .12)$ in the Blue Moon MD method. We define a quantity $W(M)$ as:

$$
W(M)=-\ln \frac{\left\langle\theta_{A}\left(\mathbf{x}_{0}\right) \theta_{B}\left(\mathbf{x}_{M}\right)\right\rangle}{\left\langle\theta_{A}\left(\mathbf{x}_{0}\right)\right\rangle}
$$

This can be regarded as the reversible work needed to make the trajectory end in region B at $t=M \delta t$. We can find it through thermodynamic integration. First we define a succession of regions $B_{\lambda}$, where $\lambda$ is a parameter, with $B_{\lambda}$ at $\lambda=0$ spanning the whole of phase space, and with $B_{\lambda}=B$ for $\lambda=1$. The quantity $W(M)$ will then be:

$$
W(M)=-\int_{0}^{1} \frac{d}{d \lambda} \ln \left\langle\theta_{A}\left(\mathbf{x}_{0}\right) \theta_{B_{\Lambda}}\left(\mathbf{x}_{M}\right)\right\rangle d \lambda
$$

This quantity can be obtained by running MD simulations for several different values of $\lambda$, and then numerically evaluating (2.5.33). It is also possible to obtain it by using constrained dynamics, as in the Blue Moon method.

Putting everything together, we can write the procedure for finding a reaction rate using TPS as:

1. Obtain an initial reacting trajectory, for example, by running a standard MD simulation at a much higher temperature or using an action-based method. Define the length of the path $t_{F}$ and the number of slices $M$, and the reactant and product regions $\mathrm{A}$ and $\mathrm{B}$.

2. Generate new paths by slightly modifying the previous path. This may be done using the shooting and shifting moves described earlier. 
3. For each of these paths, obtain the quantity $\left\langle\dot{\theta}_{B}\left(\mathbf{x}_{\tau}\right)\right\rangle_{A B}$. If this function does not attain a stationary value, restart the simulation using a longer path length.

4. Run a set of MD simulations for different end regions $B_{\lambda}$, and numerically obtain the integrand in (2.5.33). Then integrate (2.5.33) numerically to obtain the reversible work $W(M)$.

5. Using the results from steps 3 and 4 , obtain the reaction rate from equation (2.5.31).

A detailed and very clear description of how to find reaction rates using TPS can be found in ref. 468. A description of a new modified method to evaluate reaction rates, transition interface sampling, can be found in ref 470. The major advantages of the TPS methodology are the fact that it can generate real dynamic trajectories, which can be used to compute time correlation functions, and the fact that it does not require defining a reaction coordinate, or identifying an energy barrier for the reaction. The TPS trajectories are not biased by imposing an artificial restriction on the initial conditions, and thus better represent the real behavior of the system. This, however, has to be balanced against the increased computational cost of TPS ${ }^{471}$.

The trajectories generated with TPS can be used to find the transition state regions of configurational space, and to determine proper reaction coordinates for the system. In order to determine the transition state region, one may take TPS trajectories and, starting from a given time slice, generate several dynamic trajectories by assigning initial velocities from a Maxwell-Boltzmann probability distribution. If the time slice selected corresponds to the transition state region, the probability of ending in either basin of attraction should be close to $1 / 2$. Further descriptions of how to define transition states and reaction coordinates using TPS results can be found in refs. 468,472-474. 
The TPS methodology is relatively new, and hence there are not as many applications in the literature. Some recent representative applications of TPS are:

- A study of the reaction mechanism for the autoionization of liquid water using TPS in combination with $\mathrm{CPMD}^{475}$. This simple reaction, which is key to the understanding of acid-base chemistry, has actually a complicated mechanism involving the hydrogenbonded network of liquid water.

- A study of the reaction between the ferrous ion and hydrogen peroxide in aqueous solution using TPS in combination with $\mathrm{CPMD}^{476}$. In this work TPS is used to elucidate the reaction mechanism, which involves complex solvent effects.

- An analysis of the folding pathways of $\beta$-hairpin folding of aqueous G-B1 protein, using TPS together with the CHARMM force field ${ }^{477}$. In this work the proper order parameters to define the stable states are found for this system, and the rates for the transitions involved in the folding are estimated.

- A study of the kinetic pathways for single base-pair binding/unbinding in DNA, using the CHARMM force field ${ }^{478}$. In this work the free energy surface for a model DNA sequence in aqueous solution at $300 \mathrm{~K}$, and proper reaction coordinates and the transition state region are found for the base pairing/unpairing.

\subsection{Concluding Remarks.}

In recent years, many methods have been developed for the study of the dynamics of different chemical reactions. To this date, there are several approaches available for the computation of rate constants, depending on the complexity of the reaction studied, how well its mechanism is understood, and how long is the characteristic time associated with it. From the basic principles of quantum chemistry, we have the $a b$ initio methods, which make it possible for us to understand how a reaction proceeds without much previous 
knowledge. The CPMD method even provides a way of looking at the dynamics of a reaction from first principles. The computational cost of such methods, however, makes them prohibitive for the study of complex systems where a large number of atoms has to be considered. We have also reviewed methods that allow us to handle the separation of time scales typical of reaction processes, for both the cases when the mechanism is well understood (TST, reactive flux method, blue moon MD) and the cases where it is not (TPS). Although these methods are relatively new, they promise to be very useful. They allow us to bridge the gap between the extremely detailed, short-time description of CPMD and the much longer-time description required for studying activated processes. In several recent applications, the CPMD method has been successfully combined with the Blue Moon MD and TPS techniques to obtain accurate pictures of reactive processes.

There are still major limitations to the types of systems that can be handled using current state-of-the-art techniques. Many chemical reactions of interest to both scientists and engineers (e.g. polymerizations, biological reactions) are not simple enough to be treated with a method like CPMD, and can be expected to be still too complex for many years. Force field methods offer a way around this, but they cannot be used as predictive tools as $a b$ initio methods can. The intermediate approach of $\mathrm{QM} / \mathrm{MM}$ is the most likely to be successful as a predictive tool for such complex systems.

There is a definite need to develop methods that simplify the problem of finding potential energies. The CPMD method is a big step in this direction, but still has serious limitations. It is also necessary to refine the methods for handling differing time scales, so they can be applied to larger systems without prohibitive computational costs. Nevertheless, we have seen a big increase in the complexity of the systems that can be studied in the recent past, an increase due more to the development of better simulation techniques than to the development of better computers. If this trend continues, we can expect to have much better tools in the near future. 


\section{References and Notes}

\footnotetext{
${ }^{1}$ Santiso, E.E., Gubbins, K.E. (2004) “Multi-Scale Molecular Modeling of Chemical Reactivity”, Mol. Simulat. 30, 699 (2004).

${ }^{2}$ Truhlar, D. (1981) Potential Energy Surfaces and Dynamics Calculations for Chemical Reactions and Molecular Energy Transfer (Plenum Press, New York)

${ }^{3}$ Simons, J. (2003) An Introduction to Theoretical Chemistry (Cambridge University Press, Cambridge)

${ }^{4}$ Leach, A.R. (2001) Molecular Modeling. Principles and Applications, $2^{\text {nd }}$ ed (Prentice Hall, Harlow)

${ }^{5}$ Jensen, F. (1999) Introduction to Computational Chemistry (John Wiley and Sons, Chichester)

${ }^{6}$ Hirst, D.M. (1985) Potential Energy Surfaces: Molecular Structure and Reaction Dynamics (Taylor and Francis, London)

${ }^{7}$ Szabo A., Ostlund, N. (1996) Modern Quantum Chemistry: Introduction to Advanced Electronic Structure Theory (Dover, New York)

${ }^{8}$ McQuarrie, D.A., Simon, J.D., Simon, J.D., Choi, J. (1997) Physical Chemistry: A Molecular Approach (University Science Books, Sausalito)

${ }^{9}$ Simons, J. (1991) “An experimental chemist's guide to ab initio quantum chemistry”, J. Phys. Chem. 95, 1017.

${ }^{10}$ Thijssen, J.M. (1999) Computational Physics (Cambridge University Press, Cambridge)

${ }^{11}$ Frenkel, D., Smit, B. (2002) Understanding Molecular Simulation: From Algorithms to Applications, $2^{\text {nd }}$ ed. (Academic Press, London)

${ }^{12}$ Allen, M.P., Tildesley, D.J. (1987) Computer Simulation of Liquids (Clarendon Press, Oxford)

${ }^{13}$ Connors, K.A. (1990) Chemical Kinetics: The Study of Reaction Rates in Solution (VCH Publishers, New York)

${ }^{14}$ Laidler, K.J. (1969) Theories of Chemical Reaction Rates (McGraw-Hill, New York)

${ }^{15}$ Glasstone, S., Laidler, K.J., Eyring, H. (1941) The Theory of Rate Processes (McGraw-Hill, New York)
} 
${ }^{16}$ For several examples of this kind of system, see ref 6, chapter 3.

${ }^{17}$ Sprik, M. (2000) "Computation of the pK of liquid water using coordination constraints", Chem. Phys. 258, 139.

${ }^{18}$ Sprik, M. (1998) "Coordination numbers as reaction coordinates in constrained molecular dynamics", Faraday Discuss. 110, 437.

${ }^{19}$ Gertner, B.J., Hynes, J.T. (1998) "Model molecular dynamics simulation of hydrochloric acid ionization at the surface of stratospheric ice", Faraday Discuss. 110, 301.

${ }^{20}$ Geissler, P.L., Dellago, C., Chandler, D. (1999) “Kinetic pathways of ion pair dissociation in water”, J. Phys. Chem. B 103, 3706.

${ }^{21}$ See section 2.4.10, also see refs. 331, 332 .

${ }^{22}$ Fukui, K. (1970) “A formulation of reaction coordinate”, J. Phys. Chem. 74, 4161.

${ }^{23}$ Fukui, K., Kato, S., Fujimoto, H. (1975) "Constituent analysis of the potential gradient along a reaction coordinate. Method and an application to $\mathrm{CH}_{4}+\mathrm{T}$ reaction", J. Am. Chem. Soc. 97, 1 .

${ }^{24}$ Fukui, K. (1981) “The path of chemical reactions - The IRC approach”, Acc. Chem. Res. 14, 363.

${ }^{25}$ The Hessian is the matrix of second derivatives of the energy with respect to the configurational variables. It is also often called the dynamical matrix.

${ }^{26}$ For a system with $N$ degrees of freedom, there are $N(N+1) / 2$ distinct elements in the Hessian matrix. The numerical estimation of each derivative would require at least two single-point calculations using, for example, an $a b$ initio method. This can be prohibitive for all but the simplest systems.

${ }^{27}$ Schinke, R., Lester Jr. , W.A. (1979) “Trajectory study of O $+\mathrm{H}_{2}$ reactions fitted $a b$ initio surfaces I: Triplet case", J. Chem. Phys. 70, 4893

${ }^{28}$ An exception to this is transition path sampling, which does not require a previous knowledge of the transition state. This method is discussed in section 5.

${ }^{29}$ Fletcher, R. (1987) Practical Methods of Optimization, $2^{\text {nd }}$ ed (Wiley, New York)

${ }^{30}$ Gill, P.E., Murray, W., Wright, M.H. (1981) Practical Optimization (Academic Press, New York) 
${ }^{31}$ Schlegel, H.B. (2003) "Exploring potential energy surfaces for chemical reactions: An overview of some practical methods", J. Comput. Chem. 24, 1514.

${ }^{32}$ Broyden, C.G. (1970) "The convergence of a class of double-rank minimization algorithms 1. General considerations", J. Inst. Math. Applicat. 6, 76.

${ }^{33}$ Broyden, C.G. (1970) "The convergence of a class of double-rank minimization algorithms 2 . The new algorithm", J. Inst. Math. Applicat. 6, 222.

${ }^{34}$ Fletcher, R. (1970) “A new approach to variable metric algorithms”, Comput. J. 13, 317.

${ }^{35}$ Goldfarb, D. (1970) “A family of variable-metric methods derived by variational means”, Math. Comput. 24, 23.

${ }^{36}$ Shanno, D.F. (1970) "Conditioning of quasi-Newton methods for function minimization", Math. Comput. 24, 647.

${ }^{37}$ A. Banerjee, N. Adams, J. Simons, R. Shepard (1985) "Search for stationary points on surfaces", J. Phys. Chem. 89, 52.

${ }^{38}$ Liu, D.C., Nocedal J. (1989) “On the limited memory BFGS method for large-scale optimization”, Math. Prog. 45, 503.

${ }^{39}$ Nocedal, J. (1980) “Updating quasi-Newton matrices with limited storage”, Math. Comput. 35, 773.

${ }^{40}$ Murrell, J.N., Carter, S., Farantos, S.C., Huxley, P., Varandas, A.J.C. (1984) Molecular Potential Energy Functions (Wiley, New York)

${ }^{41}$ Jasien, P.G., Shepard, R. (1988) “A general polyatomic potential energy surface fitting method”, Int. J. Quantum Chem. Quantum Chem. Symp. 22, 183.

${ }^{42}$ Downing, J.W., Michi, J. “Interpolation of Multidimensional Potential Surfaces by Polynomial Roots”, In: Truhlar D., ed, Potential Energy Surfaces and Dynamics Calculations for Chemical Reactions and Molecular Energy Transfer (Plenum Press, New York)

${ }^{43}$ Hollebeek, T., Ho, T.-S., Rabitz, H. (1999) “Constructing multidimensional molecular potential energy surfaces from ab initio data", Annu. Rev. Phys. Chem. 50, 537. 
${ }^{44}$ Ischtwan, J., Collins, M.A. (1994) "Molecular potential energy surfaces by interpolation”, J. Chem. Phys. 100, 8080 .

${ }^{45}$ Jordan, M.J.T., Thompson, K.C., Collins, M.A. (1995) “Convergence of molecular potential energy surfaces by interpolation: Application to the $\mathrm{OH}+\mathrm{H}_{2} \rightarrow \mathrm{H}_{2} \mathrm{O}+\mathrm{H}$ reaction”, J. Chem. Phys. 102, 5647. ${ }^{46}$ Jordan, M.J.T., Thompson, K.C., Collins, M.A. (1995) “The utility of higher order derivatives in constructing molecular potential energy surfaces by interpolation”, J. Chem. Phys. 103, 9669.

${ }^{47}$ Thompson, K.C., Jordan, M.J.T., Collins, M.A. (1998) “Molecular potential energy surfaces by interpolation in Cartesian coordinates", J. Chem. Phys. 108, 564.

${ }^{48}$ Thompson, K.C., Jordan, M.J.T., Collins, M.A. (1998) "Polyatomic molecular potential energy surfaces by interpolation in local internal coordinates", J. Chem. Phys. 108, 8302.

${ }^{49}$ Bettens, R.P.A., Collins, M.A. (1999) "Learning to interpolate molecular potential energy surfaces with confidence: A Bayesian approach", J. Chem. Phys. 111, 816.

${ }^{50} \mathrm{Wu}, \mathrm{T}$., Manthe, I. (2003) “A potential energy surface construction scheme for accurate reaction rate calculations: General approach and a test for the $\mathrm{H}+\mathrm{CH}_{4} \rightarrow \mathrm{H}_{2}+\mathrm{CH}_{3}$ ”, J. Chem. Phys. 119, 14.

${ }^{51}$ Ayala, P.Y., Schlegel, H.B. (1997) “A combined method for determining reaction paths, minima, and transition state geometries", J. Chem. Phys. 107, 375.

${ }^{52}$ Elber, R., Karplus, M. (1987) “A method for determining reaction paths in large molecules: Application to myoglobin”, Chem. Phys. Lett. 139, 375.

${ }^{53}$ Czerminski, R., Elber, R. (1990) "Reaction path study of conformational transitions in flexible systems: Applications to peptides", J. Chem. Phys. 92, 5580.

${ }^{54}$ Jónsson, H., Mills, G., Jacobsen, K.W. (1998) “Nudged Elastic Band Method for Finding Minimum Energy Paths of Transitions”, In: Berne, B.J., Ciccotti G., Coker, D.F., eds, Computer Simulation of Rare Events and Dynamics of Classical and Quantum Condensed-Phase Systems - Classical and Quantum Dynamics in Condensed Phase Simulations, (World Scientific, Singapore); see references therein for related methods. 
${ }^{55}$ Ponder, J.W., Case, D.A. (2003) "Force fields for protein simulations", Adv. Prot. Chem. 66, 27.

${ }^{56}$ Hünenberger, P.H., van Gunsteren, W.F. (1997) “Empirical Classical Force Fields for Molecular Systems”, In: van Gunsteren, W.F., Weiner, P.K., Wilkinson, A.J., eds, Computer Simulations of Biomolecular Systems, (Kluwer Academic Publishers, Dordrecht, Netherlands)

${ }^{57}$ MacKerell Jr., A.D. (2001) “Atomistic Models and Force Fields”, In: Becker, O., MacKerell Jr., A.D., Roux B., Watanabe, M., eds, Computational Biochemistry and Biophysics, (Marcel Dekker Inc., New York)

${ }^{58}$ Orozco, M., Pérez, A., Noy, A., Luque, F.J. (2003) “Theoretical methods for the simulation of nucleic acids", Chem. Soc. Rev. 32, 350.

${ }^{59}$ Note that this also requires a way to figure out which atoms are bonded. This can be an input to the method or it can be calculated using some empirical equations.

${ }^{60}$ Morse, P.M. (1929) "Diatomic molecules according to the wave mechanics. II. Vibrational levels", Phys. Rev. 34, 57.

${ }^{61}$ van Duin, A.C.T., Dasgupta, S., Lorant, F., Goddard III, W.A. (2001) "ReaxFF: A reactive force field for hydrocarbons", J. Phys. Chem. A 105, 9396.

${ }^{62}$ This is also true for the stretching and valence angle terms. Sometimes the energy penalty due to the outof-plane bending of an $s p^{2}$-hibridized molecule is added separately to the energy expansion.

${ }^{63}$ London, F. (1930) "Zur theorie und systematik der molekularkräfte”, Z. Phys. 63, 245.

${ }^{64}$ London, F. (1930) “Über einige eigenschaften und anwendungen der molekularkräfte”, Z. Phys. Chem. B $11,222$.

${ }^{65}$ London, F. (1937) “The general theory of molecular forces”, Trans. Faraday Soc. 33, 8.

${ }^{66}$ Stone, A.J. (1996) The Theory of Intermolecular Forces (Clarendon Press, Oxford)

${ }^{67}$ Buckingham, R.A. (1938) “The classical equation of state of gaseous Helium, Neon and Argon”, Proc. Roy. Soc. London A 168, 264. 
${ }^{68}$ Morley, S.D., Abraham, R.J., Haworth, I.S., Jackson, D.E., Saunders, M.R., Vinter, J.G. (1991)

"COSMIC(90) - An improved molecular mechanics treatment of hydrocarbons and conjugated systems", $J$. Comput.-Aid. Mol. Des. 5, 475.

${ }^{69}$ van Duin, A.C.T., Strachan, A., Stewman, S., Zhang, Q., Xu, X., Goddard III, W.A. (2003) "ReaxFF ${ }_{\mathrm{SiO}}$ reactive force field for silicon and silicon oxide systems", J. Phys. Chem. A 107, 3803.

${ }^{70}$ Rappé, A.K., Goddard, W.A. (1991) “Charge equilibration for molecular dynamics simulations”, J. Phys. Chem. 95, 3358.

${ }^{71}$ Mortier, W.J., Ghosh, S.K., Shankar, S. (1986) "Electronegativity equalization method for the calculation of atomic charges in molecules", J. Am. Chem. Soc. 108, 4315.

${ }^{72}$ Janssens, G.O.A., Baekelandt, B.G., Toufar, H., Mortier, W.J., Schoonheydt, R.A. (1995) “Comparison of cluster and infinite crystal calculations on zeolites with the electronegativity equalization method (EEM)", $J$. Phys. Chem. 99, 3251.

${ }^{73}$ This list is by no means complete. For more comprehensive lists of FFs see, for example, refs. 4, 5 and 55.

${ }^{74}$ Allinger, N.L. (1977) "Conformational analysis. 130. MM2. A hydrocarbon force field utilizing $\mathrm{V}_{1}$ and $\mathrm{V}_{2}$ torsional terms", J. Am. Chem. Soc. 99, 8127.

${ }^{75}$ Allinger, N.L., Zhou, X.F., Bergsma, J. (1994) “Molecular mechanics parameters”, J. Mol. Struct. Theochem 118, 69.

${ }^{76}$ Allinger, N.L., Yuh, Y.H., Lii, J.H. (1989) “Molecular mechanics. The MM3 force field for hydrocarbons. 1", J. Am. Chem. Soc. 111, 8551.

${ }^{77}$ Lii, J.H., Allinger, N.L. (1989) "Molecular mechanics. The MM3 force field for hydrocarbons. 2. Vibrational frequencies and thermodynamics", J. Am. Chem. Soc. 111, 8566.

${ }^{78}$ Lii, J.H., Allinger, N.L. (1989) "Molecular mechanics. The MM3 force field for hydrocarbons. 3. The van der Waals' potentials and crystal data for aliphatic and aromatic hydrocarbons", J. Am. Chem. Soc. 111, 8576. 
${ }^{79}$ Allinger, N.L., Chen, K., Lii, J.H. (1996) “An improved force field (MM4) for saturated hydrocarbons”, J. Comput. Chem. 17, 642.

${ }^{80}$ Nevins, N., Chen, K., Allinger, N.L. (1996) “Molecular mechanics (MM4) calculations on alkenes”, J. Comput. Chem. 17, 669.

${ }^{81}$ Nevins, N., Lii, J.H., Allinger, N.L. (1996) “Molecular mechanics (MM4) calculations on conjugated hydrocarbons", J. Comput. Chem. 17, 695.

${ }^{82}$ Nevins, N., Allinger, N.L. (1996) “Molecular mechanics (MM4) vibrational frequency calculations for alkenes and conjugated hydrocarbons", J. Comput. Chem. 17, 730.

${ }^{83}$ Allinger, N.L., Chen, K., Katzenellenbogen, J.A., Wilson, S.R., Anstead, G.M. (1996) “Hyper conjugative effects on carbon-carbon bond lengths in molecular mechanics (MM4)", J. Comput. Chem. 17, 747. ${ }^{84}$ Halgren, T.A. (1996) “Merck molecular force field. 1. Basis, form, scope, parameterization, and performance of MMFF94", J. Comput. Chem. 17, 490.

${ }^{85}$ Halgren, T.A. (1996) "Merck molecular force field. 2. MMFF94 van der Waals and electrostatic parameters for intermolecular interactions", J. Comput. Chem. 17, 520.

${ }^{86}$ Halgren, T.A. (1996) "Merck molecular force field. 3. Molecular geometries and vibrational frequencies for MMFF94”, J. Comput. Chem. 17, 553.

${ }^{87}$ Halgren, T.A. (1996) "Merck molecular force field. 4. Conformational energies and geometries for MMFF94", J. Comput. Chem. 17, 587.

${ }^{88}$ Halgren, T.A. (1996) "Merck molecular force field. 5. Extension of MMFF94 using experimental data, additional computational data, and empirical rules”, J. Comput. Chem. 17, 616.

${ }^{89}$ Weiner, P.K., Kollman, P.A. (1981) “AMBER: Assisted model building with energy refinement. A general program for modeling molecules and their interactions", J. Comput. Chem. 2, 287.

${ }^{90}$ Cornell, W.D., Cieplak, P., Bayly, C.I., Gould, I.R., Merz Jr., K.M., Ferguson, D.M., Spellmeyer, D.C., Fox, T., Caldwell, J.W., Kollman, P.A. (1995) “A second generation force field for the simulation of proteins, nucleic acids, and organic molecules”, J. Am. Chem. Soc. 117, 5179. 
${ }^{91}$ For a history of the development of the AMBER, OPLS and CHARMM force fields, as well as a more complete list of references, see ref. ${ }^{55}$.

${ }^{92}$ Damm, W., Frontera, A., Tirado-Rives, J., Jorgensen, W.L. (1997) “OPLS all-atom force field for carbohydrates", J. Comput. Chem. 18, 1955.

${ }^{93}$ Jorgensen, W.L. (1998) “OPLS Force Fields”, In: von Rague Schleyer, P., Allinger, N.L., Clark, T., Gasteiger, J., Kollman, P.A., Schaefer III, H.F., Schreiner, P.R., eds, Encyclopedia of Computational Chemistry (John Wiley \& Sons, Chichester)

${ }^{94}$ Brooks, R., Bruccoleri, R.E., Olafson, B.D., States, D.J., Swaminathan, S., Karplus, M. (1983)

"CHARMM: A program for macromolecular energy, minimization, and dynamics calculations", J. Comput. Chem. 4, 187.

${ }^{95}$ MacKerell Jr., A.D., Brooks, B., Brooks III, C.L., Nilsson, L., Roux, B., Won, Y., Karplus, M. (1998)

"CHARMM: The Energy Function and its Parameterization with an Overview of the Program", In: von Rague Schleyer, P., Allinger, N.L., Clark, T., Gasteiger, J., Kollman, P.A. Schaefer III, H.F., Schreiner, P.R. , eds, Encyclopedia of Computational Chemistry (John Wiley \& Sons, Chichester)

${ }^{96}$ van Gunsteren, W.F., Berendsen, H.J.C. (1987) Groningen Molecular Simulation (GROMOS) Library Manual (University of Groningen, Netherlands)

${ }^{97}$ van Gunsteren, W.F., Daura, X., Mark, A.E. (1998) “The GROMOS Force Field”, In: von Rague Schleyer, P., Allinger, N.L., Clark, T., Gasteiger, J., Kollman, P.A., Schaefer III, H.F., Schreiner, P.R., eds, Encyclopedia of Computational Chemistry, (John Wiley \& Sons, Chichester)

${ }^{98}$ Scott, W.R.P., Hünenberger, P.H., Tironi, I.G., Mark, A.E., Billeter, S.R., Fennen, J., Torda, A.E., Huber, T., Krüger, P., van Gunsteren, W.F. (1999) “The GROMOS biomolecular simulation program package”, J. Phys. Chem. A 103, 3596.

${ }^{99}$ Némethy, G., Pottle, M.S., Scheraga, H.A. (1983) “Energy parameters in polypeptides. 9. Updating of geometrical parameters, nonbonded interactions, and hydrogen bond interactions for the naturally occurring amino acids", J. Phys. Chem. 87, 1883. 
${ }^{100}$ Némethy, G., Gibson, K.D., Palmer, K.A., Yoon, C.N., Paterlini, G., Zagari, A., Rumsey, S., Scheraga, H.A. (1992) "Energy parameters in polypeptides. 10. Improved geometrical parameters and nonbonded interactions for use in the ECEPP/3 algorithm, with application to proline-containing peptides", J. Phys. Chem. 96, 6472.

${ }^{101}$ Ripoll, D.R., Scheraga, H.A. (1998) “ECEPP: Empirical Conformational Energy Program for Peptides”, In: von Rague Schleyer, P., Allinger, N.L., Clark, T., Gasteiger, J., Kollman, P.A., Schaefer III, H.F., Schreiner, P.R., eds, Encyclopedia of Computational Chemistry (John Wiley \& Sons, Chichester) ${ }^{102}$ Mayo, S.L., Olafson, B.D., Goddard III, W.A. (1990) “DREIDING: a generic force field for molecular simulations", J. Phys. Chem. 94, 8897.

${ }^{103}$ Comba, P., Hambley, T.W. (2001) Molecular Modeling of Inorganic Compounds (Wiley-VCH, Weinheim)

${ }^{104}$ Allured, V.S., Kelly, C.M., Landis, C.R. (1991) "SHAPES empirical force field: new treatment of angular potentials and its application to square-planar transition-metal complexes”, J. Am. Chem. Soc. 113, 1.

${ }^{105}$ Dillen, J.L.M. (1995) “An empirical force field. 1. Alkanes”, J. Comput. Chem. 16, 595.

${ }^{106}$ Dillen, J.L.M. (1995) “An empirical force field. 2. Crystalline alkanes”, J. Comput. Chem. 16, 610.

${ }^{107}$ Brenner, D.W. (1990) "Empirical potential for hydrocarbons for use in simulating the chemical vapor deposition of diamond films", Phys. Rev. B 42, 9458.

${ }^{108}$ Stuart, S.J., Tutein, A.B., Harrison, J.A. (2000) “A reactive potential for hydrocarbons with intermolecular interaction", J. Chem. Phys. 112, 6472.

${ }^{109}$ Rappé, A.K., Casewit, C.J., Colwell, K.S., Goddard III, W.A., Skiff, W.M. (1992) “UFF, a full periodic table force field for molecular mechanics and molecular dynamics simulations", J. Am. Chem. Soc. 114, 10024.

${ }^{110}$ Rappé, A.K., Pietsch, M.A., Wiser, D.C., Hart, J.R., Bormann, L.M., Skiff, W.M. (1997) “RFF, conceptual development of a full periodic table force field for studying reaction potential surfaces”, J. Mol. Eng. 7, 385. 
${ }^{111}$ Eksterowicz, J.E., Houk, K.N. (1993) “Transition-state modeling with empirical force fields”, Chem. Rev. 93, 2439.

112 Jensen, F. (2003) “Using force field methods for locating transition structures”, J. Chem. Phys. 119, 8804.

${ }^{113}$ Jensen, F., Norrby, P.-O. (2003) “Transition states from empirical force fields”, Theor. Chem. Acc. 109, 1.

${ }^{114}$ Jensen, F. (1994) “Transition structure modeling by intersecting potential energy surfaces”, J. Comput.

Chem. 15, 1199.

115 Jensen, F. (1992) "Locating minima on seams of intersecting potential energy surfaces. An application to transition structure modeling”, J. Am. Chem. Soc. 114, 1596.

${ }^{116}$ Kim, Y., Corchado, J.C., Villà, J., Xing, J., Truhlar, D.G. (2000) “Multiconfiguration molecular mechanics algorithm for potential energy surfaces of chemical reactions", J. Chem. Phys. 112, 2718.

${ }^{117}$ Albu, T.V., Corchado, J.C., Truhlar, D.G. (2001) "Molecular mechanics for chemical reactions: A standard strategy for using multiconfiguration molecular mechanics for variational transition state theory with optimized multidimensional tunneling", J. Phys. Chem. A 105, 8465.

${ }^{118}$ Norrby, P.-O. (2000) "Selectivity in asymmetric synthesis from QM-guided molecular mechanics”, J. Mol. Struct. Theochem 506, 9.

${ }^{119}$ Bernardi, F., Olivucci, M., Robb, M.A. (1992) "Simulation of MC-SCF results on covalent organic multibond reactions: Molecular mechanics with valence bond (MM-VB)", J. Am. Chem. Soc. 114, 1606.

${ }^{120}$ Bearpark, M.J., Robb, M.A. (1994) "Molecular mechanics valence-bond methods for large active spaces: Application to conjugated polycyclic hydrocarbons", Chem. Phys. Lett. 217, 513.

${ }^{121}$ Mo, Y., Gao, J. (2000) “An ab initio molecular orbital - valence bond (MOVB) method for simulating chemical reactions in solution", J. Phys. Chem. A 104, 3012.

${ }^{122}$ Mo, Y., Gao, J. (2000) “Ab initio QM/MM simulations with a molecular orbital - valence bond (MOVB) method: Application to an $\mathrm{S}_{\mathrm{N}} 2$ reaction in water", J. Comput. Chem. 21, 1458. 
${ }^{123}$ Gundertofte, K., Palm, J., Pettersson, I., Stamvik, A. (1991) “A comparison of conformational energies calculated by molecular mechanics (MM2(85), SYBYL-5.1, SYBYL-5.21 and CHEMX) and semiempirical (AM1 and PM3) methods", J. Comput. Chem. 12, 200.

${ }^{124}$ Roterman, I.K., Gibson, K.D., Scheraga, H.A. (1989) “A comparison of the CHARMM, AMBER and ECEPP potentials for peptides. 1. Conformational predictions of the tandemly repeated peptide (ASN-ALAASN-PRO),", J. Biomol. Struct. Dyn. 7, 391.

${ }^{125}$ Roterman, I.K., Lambert, M.H., Gibson, K.D., Scheraga, H.A. (1989) “A comparison of the CHARMM, AMBER and ECEPP potentials for peptides. 2. Phi-psi maps for n-acetyl alanine n'-methyl amide: comparisons, contrasts and simple experimental tests”, J. Biomol. Struct. Dyn. 7, 421.

${ }^{126}$ Teeter, M.M., Case, D.A. (1990) "Harmonic and quasiharmonic descriptions of crambin”, J. Phys. Chem. 94, 8091.

${ }^{127}$ Kini, R.M., Evans, H.J. (1992) “Comparison of protein models minimized by the all-atom and unitedatom models in the AMBER force field: Correlation of RMS deviation with the crystallographic R factor and size”, J. Biomol. Struct. Dyn. 10, 265.

${ }^{128}$ Kaminski, G., Jorgensen, W.L. (1996) "Performance of the AMBER94, MMFF94, and OPLS-AA force fields for modeling organic liquids", J. Phys. Chem. 100, 18010.

${ }^{129}$ Gundertofte, K., Liljefors, T., Norrby, P.-O., Pettersson, I. (1996) “A comparison of the conformational energies calculated by several molecular mechanics methods", J. Comput. Chem. 17, 429.

${ }^{130}$ Hobza, P., Kabeláč, M., Šponer, J., Mejzlík, P., Vondrášek, J. (1997) “Performance of empirical potentials (AMBER, CFF95, CVFF, CHARMM, OPLS, POLTEV), semiempirical quantum chemical methods (AM1, MNDO/M, PM3), and ab initio Hartree-Fock method for interaction of DNA bases: Comparison with nonempirical beyond Hartree-Fock results", J. Comput. Chem. 18, 1136.

${ }^{131}$ Ceccarelli, M., Marchi, M. (1997) “Simulation of a protein crystal at constant pressure”, J. Phys. Chem. B $101,2105$. 
${ }^{132}$ Pérez, S., Imberty, A., Engelsen, S.B., Gruza, J., Mazeau, K., Jimenez-Barbero, J., Poveda, A., Espinosa, J.-F., van Eyck, B.P., Johnson, G., French, A.D., Kouwijzer, M.L.C.E., Grootenuis, P.D.J., Bernardi, A., Raimondi, L., Senderowitz, H., Durier, V., Vergoten, G., Rasmussen, K. (1998) “A comparison and chemometric analysis of several molecular mechanics force fields and parameter sets applied to carbohydrates", Carbohyd. Res. 314, 141.

${ }^{133}$ Price, D.J., Brooks III, C.L. (2002) "Modern protein force fields behave comparably in molecular dynamics simulations", J. Comput. Chem. 23, 1045.

${ }^{134}$ Reddy, S.Y., Leclerc, F., Karplus, M. (2003) “DNA polymorphism: A comparison of force fields for nucleic acids", Biophys. J. 84, 1421.

${ }^{135}$ Hu, H., Elstner, M., Hermans, J. (2003) “Comparison of a QM/MM force field and molecular mechanics force fields in simulations of alanine and glycine "dipeptides" (Ace-Ala-Nme and Ace-Gly-Nme) in water in relation to the problem of modeling the unfolded peptide backbone in solution”, Proteins 50, 451.

${ }^{136}$ Zhou, R.H. (2003) "Free energy landscape of protein folding in water: Explicit vs. implicit solvent”, Proteins, 53, 148.

${ }^{137}$ M. Patra, M. Karttunen (In press) "Systematic comparison of force fields for microscopic simulations of $\mathrm{NaCl}$ in aqueous solutions: Diffusion, free energy of hydration and structural properties". A preprint is available online at http://www.arxiv.org/PS_cache/physics/pdf/0211/0211059.pdf

${ }^{138}$ Ford, M.G., Weimar, T., Köhli, T., Woods, R.J. (2003) “Molecular dynamics simulations of galectin-1oligosaccharide complexes reveal the molecular basis for ligand diversity”, Proteins 53, 229.

${ }^{139}$ Bingham, N.C., Smith, N.E.C., Cross, T.A., Busath, D.D. (2003) "Molecular dynamics simulations of Trp side-chain conformational flexibility in the gramicidin A channel”, Biopolymers 71, 593.

${ }^{140}$ Clément, M.-J., Imberty, A., Phalipon, A., Pérez, S., Simenel, C., Mulard, L.A., Delepierre, M. (2003)

"Conformational studies of the O-species polysaccharide of Shigella flexneri 5a and of four related synthetic pentasaccharide fragments using NMR and molecular modeling”, J. Biol. Chem. 278, 47928. 
${ }^{141}$ Sarzynska, J., Nilsson, L., Kulinski, T. (2003) "Effects of base substitutions in an RNA hairpin from molecular dynamics and free energy simulations", Biophys. J. 85, 3445.

${ }^{142}$ Schaefer III, H.F., ed (1977) Modern Theoretical Chemistry (Plenum Press, New York), v. III and IV.

${ }^{143}$ Lawley K.P., ed (1987) Advances in Chemical Physics (Wiley, New York), v. LXVII and LXIX.

${ }^{144}$ Makri, N. (1999) “Time-dependent quantum methods for large systems”, Annu. Rev. Phys. Chem. 50, 167.

${ }^{145}$ Althorpe, S.C., Clary, D.C. (2003) “Quantum scattering calculations on chemical reactions”, Annu. Rev. Phys. Chem. 54, 493.

${ }^{146}$ Lester Jr., W.A., Hammond, B.L. (1990) “Quantum Monte Carlo for the electronic structure of atoms and molecules", Annu. Rev. Phys. Chem. 41, 283.

${ }^{147}$ W.M.C. Foulkes, L. Mitas, R.J. Needs, G. Rajagopal (2001) “Quantum Monte Carlo simulations of solids”, Rev. Mod. Phys. 73, 33.

${ }^{148}$ Born, M., Oppenheimer, R. (1927) “Zur Quantentheorie der Molekeln”, Ann. Phys. 84, 457.

${ }^{149}$ Tully, J.C. (2000) "Perspective on “Zur Quantentheorie der Molekeln”: Born M, Oppenheimer, R (1927) Ann. Phys 84:457”, Theor. Chem. Acc. 103, 173.

${ }^{150}$ Sutcliffe, B.T. (1975) “Fundamentals of computational quantum Chemistry”, In: Diercksen, G.H.F., Sutcliffe B.T., Veillard, A., eds, Computational Techniques in Quantum Chemistry, (Reidel, Boston)

${ }^{151}$ The term "adiabatic" in this context makes reference to the absence of thermal coupling between the electrons and the nuclei, which is equivalent to the statement that the electronic states do not depend on the momenta of the nuclei. Strictly speaking, the adiabatic approximation is not exactly the same as the BO approximation, although the terms are often confused. For a detailed explanation of this point, see ref. 6 , chapter 1 , and ref. 5 , chapter 3 .

${ }^{152}$ Preston, R.K., Tully, J.C. (1971) "Effects of surface crossing in chemical reactions: The $\mathrm{H}_{3}{ }^{+}$system”, $J$. Chem. Phys. 54, 4297.

${ }^{153}$ Tully, J.C., Preston, R.K. (1971) “Trajectory surface hopping approach to nonadiabatic molecular collisions: The reaction of $\mathrm{H}^{+}$with $\mathrm{D}_{2}{ }^{\prime}$, J. Chem. Phys. 55, 562. 
${ }^{154}$ Tully, J.C. (1990) "Molecular dynamics with electronic transitions”, J. Chem. Phys. 93, 1061.

${ }^{155}$ Drukker, K. (1999) "Basics of surface hopping in mixed quantum/classical simulations", J. Comput. Phys. $153,225$.

${ }^{156}$ Butler, L.J. (1998) “Chemical reaction dynamics beyond the Born-Oppenheimer approximation”, Annu.

Rev. Phys. Chem. 49, 125.

${ }^{157}$ A Hermitian operator is one that is equal to its adjoint.

${ }^{158}$ Poirier, R., Kari, R., Csizmadia, I.G. (1985) Handbook of Gaussian Basis Sets: a Compendium for AbInitio Molecular Orbital Calculations (Elsevier, New York)

${ }^{159}$ Dunning, T.H., Hay, P.J. “Gaussian Basis Sets for Molecular Calculations”, in ref. 142, v. 3.

${ }^{160}$ Slater, J.C. (1930) “Atomic shielding constants”, Phys. Rev. 36, 57.

${ }^{161}$ Collins, J.B., Schleyer, P.R., Binkley, J.S., Pople, J.A. (1976) “Self-consistent molecular orbital methods.

XVII. Geometries and binding energies of second-row molecules. A comparison of three basis sets.”, $J$.

Chem. Phys. 64, 5142 and references therein.

${ }^{162}$ Binkley, J.S., Pople, J.A., Hehre, W.J. (1980) “Self-consistent molecular orbital methods. 21. Small splitvalence basis sets for first-row elements", J. Am. Chem. Soc. 102, 939.

${ }^{163}$ Gordon, M.S., Binkley, J.S., Pople, J.A., Pietro, W.J., Hehre, W.J. (1982) “Self-consistent molecular orbital methods. 22. Small split-valence basis sets for second-row elements", J. Am. Chem. Soc. 104, 2797. ${ }^{164}$ Pietro, W.J., Francl, M.M., Hehre, W.J., Defrees, D.J., Pople, J.A., Binkley, J.S. (1982) “Self-consistent molecular orbital methods. 24. Supplemented small split-valence basis sets for second-row elements", J. Am .Chem. Soc. 104, 5039 .

${ }^{165}$ Ditchfield, R., Hehre, W.J., Pople, J.A. (1971) “Self-consistent molecular orbital methods. IX. An extended Gaussian-type basis for molecular-orbital studies of organic molecules", J. Chem. Phys. 54, 724. ${ }^{166}$ Hehre, W.J., Ditchfield, R., Pople, J.A. (1972) “Self-consistent molecular orbital methods. XII. Further extensions of Gaussian-type basis sets for use in molecular orbital studies of organic molecules", J. Chem. Phys. 56, 2257. 
${ }^{167}$ Hariharan, P.C., Pople, J.A. (1974) “Accuracy of AH equilibrium geometries by single determinant molecular-orbital theory", Mol. Phys. 27, 209.

${ }^{168}$ Krishnan, R., Binkley, J.S., Seeger, R., Pople, J.A. (1980) “Self-consistent molecular orbital methods. XX. A basis set for correlated wave functions", J. Chem. Phys. 72, 650.

${ }^{169}$ McLean, A.D., Chandler, G.S. (1980) “Contracted Gaussian basis sets for molecular calculations. I. Second row atoms, Z=11-18”, J. Chem. Phys. 72, 5639.

${ }^{170}$ Blaudeau, J.-P., McGrath, M.P., Curtiss, L.A., Radom, L. (1997) “Extension of Gaussian-2 (G2) theory to molecules containing third-row atoms K and Ca", J. Chem. Phys. 107, 5016.

${ }^{171}$ Curtiss, L.A., McGrath, M.P., Blaudeau, J.-P., Davis, N.E., Binning Jr., R.C., Radom, L. (1995)

"Extension of Gaussian-2 theory to molecules containing third-row atoms Ga-Kr", J. Chem. Phys. 103, 6104.

${ }^{172}$ Francl, M.M., Pietro, W.J., Hehre, W.J., Binkley, J.S., Gordon, M.S., DeFrees, D.J., Pople, J.A. (1982)

"Self-consistent molecular orbital methods. XXIII. A polarization-type basis set for second-row elements", $J$. Chem. Phys. 77, 3654

${ }^{173}$ Rassolov, V.A., Pople, J.A., Ratner, M.A., Windus, T.L. (1998) “6-31G* basis set for atoms K through Zn”, J. Chem. Phys. 109, 1223.

${ }^{174}$ Rassolov, V.A., Ratner, M.A., Pople, J.A., Redfern, P.C., Curtiss, L.A. (2001) “6-31G* basis set for thirdrow atoms", J. Comput. Chem. 22, 976.

${ }^{175}$ Petersson, G.A., Bennett, A., Tensfeldt, T.G., Al-Laham, M.A., Shirley, W.A. (1988) “A complete basis set model chemistry. I. The total energies of closed-shell atoms and hydrides of the first-row elements", $J$. Chem. Phys. 89, 2193.

${ }^{176}$ A Rydberg orbital is a diffuse function that describes the region of space that would be occupied by an electron in the presence of a closed-shell molecular cation. An example of a Rydberg state is that of the $\mathrm{NH}_{4}$ molecule.

${ }^{177}$ Dunning Jr., T. H. (1989) "Gaussian basis sets for use in correlated molecular calculations. I. The atoms boron through neon and hydrogen", J. Chem. Phys. 90, 1007. 
${ }^{178}$ Kendall, R. A., Dunning Jr., T. H., Harrison, R. J. (1992) "Electron affinities of the first-row atoms revisited. Systematic basis sets and wave functions", J. Chem. Phys. 96, 6796.

${ }^{179}$ Woon D. E., Dunning Jr., T. H. (1993) “Gaussian basis sets for use in correlated molecular calculations. III. The atoms aluminum through argon", J. Chem. Phys. 98, 1358 (1993)

${ }^{180}$ This is also known as the basis set superposition error. The term Pulay forces is more commonly used in the context of $a b$ initio molecular dynamics, where plane wave basis sets are most commonly used.

${ }^{181}$ Marx D., Hutter, J. (2000) “Ab Initio Molecular Dynamics: Theory and Implementation”, In: Grotendorst, J., ed, Modern Methods and Algorithms of Quantum Chemistry Proceedings, (Forschungszentrum Jülich), v. 1.

${ }^{182}$ Hartree, D.R. (1928) “The wave mechanics of an atom with a non-Coulomb central field. Part I. Theory and methods", Proc. Camb. Philos. Soc. 24, 89.

${ }^{183}$ Hartree, D.R. (1928) “The wave mechanics of an atom with a non-Coulomb central field. Part II. Some results and discussion", Proc. Camb. Philos. Soc. 24, 111.

${ }^{184}$ Fock, V. (1930) “Näherungsmethode zur Lösung der quantenmechanischen Mehrkörperproblems”, Z. Physik 61, 126.

${ }^{185}$ Slater, J.C. (1929) “The theory of complex spectra”, Phys. Rev. 34, 1293.

${ }^{186}$ In RHF calculations for open-shell systems (which are not recommendable in general), UHF calculations, and in calculations involving excited determinants, there is an additional complication because states appear that are not eigenfunctions of the electronic spin operator. This problem can be solved by using spin-adapted configurations. Discussions of this topic can be found in refs. $5,{ }^{7}$.

${ }^{187}$ Roothaan, C.C.J. (1951) "New developments in molecular orbital theory”, Rev. Mod. Phys. 23, 69.

${ }^{188}$ Zerner, M.C. (2000) "Perspective on "New developments in molecular orbital theory": Roothaan CCJ (1951) Rev. Mod. Phys. 23:69-89”, Theor. Chem. Acc. 103, 217.

${ }^{189}$ Hall, G.G. (1951) "The molecular orbital theory of chemical valency. 8. A method of calculating ionization potentials", Proc. Roy. Soc. Lond. Ser. A 205, 541. 
${ }^{190}$ This is the Mulliken, or chemist's notation for the two-electron integrals.

${ }^{191}$ There is, in fact, some correlation between the electrons included in the HF method due to the presence of the exchange energy. In the HF picture, electrons with the same spin avoid being in the same region, and a Fermi hole is said to exist around each electron. The term correlation energy refers to the energy that is not included in the HF method.

${ }^{192}$ Prencipe, M., Tribaudino, M., Nestola, F. (2003) “Charge-density analysis of spodumene ( $\left.\mathrm{LiAlSi}_{2} \mathrm{O}_{6}\right)$, from ab initio Hartree-Fock calculations", Phys. Chem. Miner. 30, 606.

${ }^{193}$ Csontos, J., Kálmán, M, Tasi, G. (2003) “Conformational analysis of melatonin at Hartree-Fock ab initio level”, J. Mol. Struct. Theochem 640, 69.

${ }^{194}$ Zhang, D.W., Xiang, Y., Zhang, J.Z.H. (2003) "New advance in computational chemistry: Full quantum mechanical ab initio computation of Streptavidin-Biotin interaction energy", J. Phys. Chem. B 107, 12039.

${ }^{195}$ Schütz, M., Hetzer, G., Werner, H.-J. (1999) “Low-order scaling local electron correlation methods. I. Linear scaling local MP2”, J. Chem. Phys. 111, 5691.

${ }^{196}$ Scuseria, G.E., Ayala, P.Y. (1999) "Linear scaling coupled cluster and perturbation theories in the atomic orbital basis", J. Chem. Phys. 111, 8330.

${ }^{197}$ Koopmans, T. (1933) “Ordering of wave functions and eigenenergies to the individual electrons of an atom", Physica 1, 104.

198 and also the energies of the occupied orbitals to the ionization potentials. For a discussion of Koopmans' theorem see, for example, refs. 3, 5, 7 .

${ }^{199}$ Pople, J.A., Binkley, J.S., Seeger, R. (1976) “Theoretical models incorporating electron correlation”, Int. J. Quantum Chem. Y-10, 1.

${ }^{200}$ Pople, J.A., Head-Gordon, M., Raghavachari, K. (1987) "Quadratic configuration interaction. A general technique for determining electron correlation energies", J. Chem. Phys. 87, 5968.

${ }^{201}$ Ross, B.O., Taylor, P.R., Siegbahn, P.E.M. (1980) “A complete active space SCF method (CASSCF) using a density matrix formulated super-CI approach", Chem. Phys. 48, 157; see also refs. 55, 143. 
${ }^{202}$ In the case of transition metals, also the $d$ states from the next-to-last layer will likely need to be included.

${ }^{203}$ Goddard III, W.A., Dunning Jr., T.H., Hunt, W.J., Hay, P.J. (1973) “Generalized valence bond description of bonding in low-lying states of molecules", Acc. Chem. Res. 6, 368 and references therein.

${ }^{204}$ Goddard III, W.A., Harding, L.B. (1978) “The description of chemical bonding from ab initio

calculations", Ann. Rev. Phys. Chem. 29, 363.

${ }^{205}$ Heitler W., London, F. (1927) “Wechselwirkung neutraler atome und homöopolare bindung nach der quantenmechanik", Z. Phys. 44, 455.

${ }^{206}$ Frenking, G. (2000) "Perspecitve on "Wechselwirkung neutraler atome und homöopolare bindung nach der quantenmechanik": Heitler W, London F (1927) Z. Phys. 44:455-472”, Theor. Chem. Acc. 103, 177. ${ }^{207}$ Gallup, G.A. (2002) Valence Bond Methods: Theory and Applications (Cambridge University Press, Cambridge)

${ }^{208}$ Cooper D.L., ed (2002) Valence Bond Theory (Elsevier, Amsterdam)

${ }^{209}$ Gerratt, J., Cooper, D.L., Karadakov, P.B., Raimondi, M. (1997) “Modern valence bond theory”, Chem. Soc. Rev. 26, 87.

${ }^{210}$ Hiberty, P.C., Shaik, S. (2002) "Two new symmetry-adapted perturbation theories for the calculation of intermolecular interaction energies", Theor. Chem. Acc. 108, 255.

${ }^{211}$ Thorsteinsson, T., Cooper, D.L., Gerratt, J., Karadakov, P.B., Raimondi, M. (1996) “Modern valence bond representations of CASSCF wavefunctions", Theor. Chem. Acc. 93, 343.

${ }^{212}$ Sakai, S. (2003) "Theoretical analysis of concerted and stepwise mechanisms of the hetero-Diels-Alder reaction of butadiene with formaldehyde and thioformaldehyde" J. Mol. Struct. Theochem 630, 177.

${ }^{213}$ Wirtz, L., Burgdörfer, J., Dallos, M., Müller, T., Lischka, H. (2003) “Potential-energy surfaces for charge exchange between singly charged ions and a LiF surface", Phys. Rev. A 68, \#032902.

${ }^{214}$ Chattopadhyaya, S., Das, K.K. (2003) "Electronic states of SiSe: a configuration interaction study", Chem. Phys. Lett. 382, 249. 
${ }^{215}$ Møller C., Plesset, M.S. (1934) “Note on an approximation treatment for many-electron systems”, Phys. Rev. 46, 618.

${ }^{216}$ Pople, J.A., Krishnan, R., Schlegel, H.B., Binkley, J.S. (1978) “Electron correlation theories and their application to study of simple reaction potential surfaces", Int. J. Quantum Chem. 14, 545.

${ }^{217}$ Bartlett, R.J., Silver, D.M. (1975) "Many-body perturbation theory applied to electron pair correlation energies. I. Closed-shell first-row diatomic hydrides", J. Chem. Phys. 62, 3258.

${ }^{218}$ Head-Gordon, M., Pople, J.A., Frisch, M.J. (1988) "MP2 energy evaluation by direct methods", Chem. Phys. Lett. 153, 503.

${ }^{219}$ Frisch, M.J., Head-Gordon, M., Pople, J.A. (1990) “A direct MP2 gradient method”, Chem. Phys. Lett. 166, 275.

${ }^{220}$ Frisch, M.J., Head-Gordon, M., Pople, J.A. (1990) “Semi-direct algorithm for the MP2 energy and gradient", Chem. Phys. Lett. 166, 281.

${ }^{221}$ Krishnan, R., Pople, J.A. (1978) “Approximate 4th-order perturbation theory of electron correlation energy", Int. J. Quantum Chem. 14, 91.

${ }^{222}$ Andersson, K., Malmqvist, P.-Å., Ross, B.O. (1992) "Second-order perturbation theory with a complete active space self-consistent field reference function”, J. Chem. Phys. 96, 1218.

${ }^{223}$ Ignatov, S.K., Sennikov, P.G., Razuvaev, A.G., Chuprov, L.A., Schrems, O., Ault, B.S. (2003)

"Theoretical study of the reaction mechanism and role of water clusters in the gas-phase hydrolysis of $\mathrm{SiCl}_{4}$ ", J. Phys. Chem. A 107, 8705.

${ }^{224}$ Enchev, V., Ivanova, G., Stoyanov, N. (2003) “Tautomeric and conformational equilibrium of 2nitrosophenol and 9,10-phenanthrenequinonemonooxime: $a b$ initio and NMR study", J. Mol. Struct. Theochem 640, 149.

${ }^{225}$ Khuong, K.S., Houk, K.N. (2003) “One-bond, two-bond, and three-bond mechanisms in thermal diazetizations of 2,3-Diazabicyclo[2.2.2]oct-2-enes, trans-Azomethane, and 2,3-Diazabicyclo[2.2.1]hept-2ene", J. Am. Chem. Soc. 125, 14867. 
${ }^{226}$ Sinanoğlu, O. (1964) "Many-electron theory of atoms, molecules and their interactions", Adv. Chem.

Phys. 6, 315.

${ }^{227}$ Nesbet, R.K. (1965) "Electronic correlation in atoms and molecules", Adv. Chem. Phys. 9, 321.

${ }^{228}$ Rigorously speaking, the pair function should also contain the contributions from the single excitations of electrons $a$ and $b$. We include only the double excitations in this discussion, which eventually leads to the CCD method.

${ }^{229}$ An unitary transformation is defined in terms of an operator whose inverse is equal to its adjoint. It is the complex equivalent of a rotation operator.

${ }^{230}$ Also triplets, quintuples, etc., which would lead to full CI. As mentioned in note 228, we consider only the even excitation contributions.

${ }^{231}$ Čižek, J. (1966) "Correlation problem in atomic and molecular systems. Calculation of wave function components in Ursell-type expansion using quantum-field theoretical methods", J. Chem. Phys. 45, 4256.

${ }^{232}$ Čižek, J. (1969) "Use of the cluster expansion and the technique of diagrams in calculations of correlation effects in atoms and molecules", Adv. Chem, Phys. 14, 35.

${ }^{233}$ The equations for CCD were derived in Čižek, J., Paldus, J. (1971) "Correlation problems in atomic and molecular systems. III. Rederivation of the coupled-pair many-electron theory using the traditional quantum chemical methods", Int. J. Quantum. Chem. 5, 359.

${ }^{234}$ Purvis III, G.D., Bartlett, R.J. (1982) “A full coupled-cluster singles and doubles model: The inclusion of disconnected triples", J. Chem. Phys. 76, 1910.

${ }^{235}$ Bartlett, R.J. (1989) “Coupled-cluster approach to molecular structure and spectra: A step toward predictive quantum chemistry", J. Phys. Chem. 93, 1697.

${ }^{236}$ Bartlett, R.J. (1981) "Many-body perturbation theory and coupled cluster theory for electron correlation in molecules", Annu. Rev. Phys. Chem. 32, 359. 
${ }^{237}$ Lee, T.J., Scuseria, G.E. (1995) “Achieving Chemical Accuracy with Coupled-Cluster Theory”, In: Langhoff, S. R., ed, Quantum Mechanical Structure Calculations with Chemical Accuracy (Kluwer Academic, Boston) ${ }^{238}$ Bartlett, R.J., ed (1997) Recent Advances in Coupled-Cluster Methods (World Scientific, New Jersey) ${ }^{239}$ Oliphant, N., Adamowicz, L. (1993) “Multireference coupled-cluster method for electronic structure of molecules", Int. Rev. Phys. Chem. 12, 339.

${ }^{240}$ Mahapatra, U.S., Datta, B., Mukherjee, D. (1999) “A size-consistent state-specific multireference coupled cluster theory: Formal developments and molecular applications", J. Chem. Phys. 110, 6171.

${ }^{241}$ Kowalski, K., Piecuch, P. (2000) “Complete set of solutions of multireference coupled-cluster equations: The state-universal formalism”, Phys. Rev. A 61, \#052506.

${ }^{242}$ Chattopadhyay, S., Mahapatra, U.S., Mukherjee, D. (1999) "Property calculations using perturbed orbitals via state-specific multireference coupled-cluster and perturbation theories", J. Chem. Phys. 111, 3820.

${ }^{243}$ See, e.g. ref. 5.

${ }^{244}$ Scuseria, G.E., Lee, T.J. (1990) “Comparison of coupled-cluster methods which include the effects of connected triple excitations", J. Chem. Phys. 93, 5851.

${ }^{245}$ Feller, D., Dixon, D.A. (2003) “A nonparametrized $a b$ initio determination of the heat of formation of hydroxylamine, $\mathrm{NH}_{2} \mathrm{OH} "$, J. Phys. Chem. A 107, 10419.

${ }^{246}$ Tobita, M., Perera, S.A., Musial, M., Bartlett, R.J., Nooijen, M., Lee, J.S. (2003) “Critical comparison of single-reference and multireference coupled-cluster methods: Geometry, harmonic frequencies, and excitation energies of $\mathrm{N}_{2} \mathrm{O}_{2} "$, J. Chem. Phys. 119, 10713.

${ }^{247}$ Sari, L., McCarthy, M.C., Schaefer III, H.F., Thaddeus, P. (2003) “Mono- and dibridged isomers of $\mathrm{Si}_{2} \mathrm{H}_{3}$ and $\mathrm{Si}_{2} \mathrm{H}_{4}$ : The true ground state global minima. Theory and experiment in concert", J. Am. Chem. Soc. 125, 11409.

${ }^{248}$ Pople, J.A., Segal, G.A. (1965) “Approximate self-consistent molecular orbital theory. 2. Calculations with complete neglect of differential overlap", J. Chem. Phys. 43, S136. 
${ }^{249}$ Pople, J.A., Segal, G.A. (1966) “Approximate self-consistent molecular orbital theory. 3. CNDO results for $\mathrm{AB}_{2}$ and $\mathrm{AB}_{3}$ systems", J. Chem. Phys. 44, 3289.

${ }^{250}$ Pople, J.A., Beveridge, D.L., Dobosh, P.A. (1967) “Approximate self-consistent molecular orbital theory. 5. Intermediate neglect of differential overlap", J. Chem. Phys. 47, 2026.

${ }^{251}$ Pople, J.A., Santry, D.P., Segal, G.A. (1965) “Approximate self-consistent molecular orbital theory. I. Invariant procedures", J. Chem. Phys. 43, S129.

${ }^{252}$ Bingham, R.C., Dewar, M.J.S., Lo, D.H. (1975) “Ground states of molecules. XXV. MINDO/3. Improved version of the MINDO semiempirical SCF-MO method", J. Am. Chem. Soc. 97, 1285.

${ }^{253}$ Bingham, R.C., Dewar, M.J.S., Lo, D.H. (1975) “Ground states of molecules. XXVI. MINDO/3 calculations for hydrocarbons", J. Am. Chem. Soc. 97, 1294.

${ }^{254}$ Bingham, R.C., Dewar, M.J.S., Lo, D.H. (1975) “Ground states of molecules. XXVII. MINDO/3 calculations for carbon, hydrogen, oxygen and nitrogen species”, J. Am. Chem. Soc. 97, 1302.

${ }^{255}$ Bingham, R.C., Dewar, M.J.S., Lo, D.H. (1975) “Ground states of molecules. XXVIII. MINDO/3 calculations for compounds containing carbon, hydrogen, fluorine, and chlorine", J. Am. Chem. Soc. 97, 1307.

${ }^{256}$ Dewar, M.J.S., Thiel, W. (1977) “Ground states of molecules. 38. The MNDO method. Approximations and parameters", J. Am. Chem. Soc. 99, 4899.

${ }^{257}$ Dewar, M.J.S., Thiel, W. (1977) “Ground states of molecules. 39. MNDO results for molecules containing hydrogen, carbon, nitrogen and oxygen”, J. Am. Chem. Soc. 99, 4907.

${ }^{258}$ Dewar, M.J.S., Zoebisch, E.G., Healy, E.F., Stewart, J.J.P. (1985) “Development and use of quantum mechanical molecular models. 76. AM1: a new general purpose quantum mechanical molecular model”, $J$. Am. Chem. Soc. 107, 3902.

${ }^{259}$ Stewart, J.J.P. (1989) “Optimization of parameters for semiempirical methods. 1. Method”, J. Comput. Chem. 10, 209. 
${ }^{260}$ Stewart, J.J.P. (1989) “Optimization of parameters for semiempirical methods. 2. Applications", J. Comput. Chem. 10, 221.

${ }^{261}$ Dewar, M.J.S., Jie, C., Yu, J. (1993) “SAM1; The first of a new series of general purpose quantum mechanical molecular models", Tetrahedron 49, 5003.

${ }^{262}$ Winget, P., Selçuki, C., Horn, A.H.C., Martin, B., Clark, T. (2003) “Towards a "next generation” neglect of diatomic differential overlap based semiempirical molecular orbital technique", Theor. Chem. Acc. 110, 254.

${ }^{263}$ Linnanto, J., Korppi-Tommola, K. (2004) "Semiempirical PM5 molecular orbital study on chlorophylls and bacteriochlorophylls: Comparison of semiempirical, ab initio, and density functional results", J. Comput. Chem. 25, 123.

${ }^{264}$ Tubert-Brohman, I., Guimarães, C.R.W., Repasky, M.P., Jorgensen, W.L. (2004) “Extension of the PDDG/PM3 and PDDG/MNDO semiempirical molecular orbital methods to the halogens", J. Comput. Chem. 25, 138.

${ }^{265}$ Bräuer, M., Kunert, M., Dinjus, E., Klußmann, M., Döring, M., Görls, H., Anders, E. (2000) “Evaluation of the accuracy of PM3, AM1 and MNDO/d as applied to zinc compounds", J. Mol. Struct. Theochem 505, 289.

${ }^{266}$ Pichierri, F. (2003) "Computation of the permanent dipole moment of $\alpha$-chymotrypsin from linear scaling semiempirical quantum mechanical methods", J. Mol. Struct. Theochem 664-665, 197.

${ }^{267}$ Tsukamoto, K., Nakamura, S., Shimizu, K. (2003) “SAM1 semiempirical calculations on the catalytic cycle of nitric oxide reductase from Fusarium oxysporum", J. Mol. Struct. Theochem, 624, 309.

${ }^{268}$ Lebedev, N.G., Zaporotskova, I.V., Chernozatonskii, L.A. (2004) "Fluorination of carbon nanotubes: Quantum chemical investigation within MNDO approximation”, Int. J. Quant. Chem. 96, 142.

${ }^{269}$ Zaporotskova, I.V., Lebedev, N.G., Chernozatonskii, L.A. (2004) "Single and regular hydrogenation and oxidation of carbon nanotubes: MNDO calculations", Int. J. Quant. Chem. 96, 149. 
${ }^{270}$ Parr, R.G., Yang, W. (1989) Density-Functional Theory of Atoms and Molecules (Oxford University Press, Oxford)

${ }^{271}$ Lundqvist, S., March, N.H. (1983) Theory of the Inhomogeneous Electron Gas (Plenum Press, New York)

${ }^{272}$ Gross, E.K.U., Dreizler, R.M. (1995) Density Functional Theory (Plenum Press, New York)

${ }^{273}$ Koch, W., Holthausen, M.C. (2000) A Chemist's Guide to Density Functional Theory (Wiley-VCH, Weinheim)

${ }^{274}$ Baerends, E.J., Gritsenko, O.V. (1997) “A quantum chemical view of density functional theory”, J. Phys. Chem. A 101, 5383.

${ }^{275}$ Hohenberg, P., Kohn, W. (1964) “Inhomogeneous electron gas”, Phys. Rev. 136, B864.

${ }^{276}$ Ernzerhof, M., Scuseria, G.E. (2000) "Perspective on "Inhomogeneous electron gas": Hohenberg P, Kohn W (1964) Phys Rev 136: B846”, Theor. Chem. Acc. 103, 259.

${ }^{277}$ Kohn, W., Sham, L.J. (1965) "Self-consistent equations including exchange and correlation effects", Phys. Rev.140, A1133.

${ }^{278}$ Baerends, E.J. (2000) "Perspective on "Self-consistent equations including exchange and correlation effects" Kohn W, Sham LJ (1965) Phys Rev A 140:133-1138”, Theor. Chem. Acc. 103, 265.

${ }^{279}$ Langreth, D.C., Mehl, M.J. (1983) "Beyond the local-density approximation in calculations of groundstate electronic properties", Phys. Rev. B 28, 1809.

${ }^{280}$ Becke, A.D.(1988) “Density-functional exchange-energy approximation with correct asymptotic behavior", Phys. Rev. A 38, 3098.

${ }^{281}$ Perdew, J.P., Burke, K., Ernzerhof, M. (1996) “Generalized gradient approximation made simple”, Phys. Rev. Lett. 77, 3865.

${ }^{282}$ Perdew, J.P., Burke, K., Ernzerhof, M. (1997) “Generalized gradient approximation made simple [Phys. Rev. Lett. 77, 3865 (1996)]”, Phys. Rev. Lett. 78, 1396. 
${ }^{283}$ Perdew, J.P., Chevary, J.A., Vosko, S.H., Jackson, K.A., Pederson, M.R., Singh, D.J., Fiolhais, C. (1992) "Atoms, molecules, solids and surfaces: Applications of the generalized gradient approximation for exchange and correlation", Phys. Rev. B 46, 6671.

${ }^{284}$ Perdew, J.P., Chevary, J.A., Vosko, S.H., Jackson, K.A., Pederson, M.R., Singh, D.J., Fiolhais, C. (1993) "Erratum: Atoms, molecules, solids and surfaces: Applications of the generalized gradient approximation for exchange and correlation", Phys. Rev. B 48, 4978.

${ }^{285}$ Perdew, J.P. (1986) "Density-functional approximation for the correlation energy of the inhomogeneous electron gas", Phys. Rev. B 33, 8822.

${ }^{286}$ Wang, Y., Perdew, J.P. (1991) “Correlation hole of the spin-polarized electron gas, with exact smallwave-vector and high-density scaling", Phys. Rev. B 44, 13298.

${ }^{287}$ Perdew, J.P., Wang, Y. (1992) “Accurate and simple analytical representation of the electron-gas correlation energy", Phys. Rev. B 45, 13244.

${ }^{288}$ Perdew, J.P., Burke, K., Wang, Y. (1996) “Generalized gradient approximation for the exchangecorrelation hole of a many-electron system", Phys. Rev. B 54, 16533.

${ }^{289}$ Lee, C., Yang, W., Parr, R.G. (1988) "Development of the Colle-Salvetti correlation-energy formula into a functional of the electron density", Phys. Rev. B 37, 785.

${ }^{290}$ Baker, J., Pulay, P. (2002) “Assessment of the Handy-Cohen optimized exchange density functional for organic reactions", J. Chem. Phys. 117, 1441.

${ }^{291}$ Baker, J., Pulay, P. (2003) “Assessment of the OLYP and O3LYP density functionals for first-row transition metals", J. Comput. Chem. 24, 1184.

${ }^{292}$ Altmann, J.A., Handy, N.C. (1999) "Evaluation of the performance of the HCTH exchange-correlation functional using a benchmark of sulfur compounds", Phys. Chem. Chem. Phys. 1, 5529.

${ }^{293}$ Handy, N.C., Cohen, A.J. (2001) “Left-right correlation energy”, Mol. Phys. 99, 403.

${ }^{294}$ Hoe, W.M., Cohen, A.J., Handy, N.C. (2001) “Assessment of a new local exchange functional OPTX”, Chem. Phys. Lett. 341, 319. 
${ }^{295}$ Handy, N.C., Cohen, A.J. (2002) “A dynamical correlation functional”, J. Chem. Phys. 116, 5411.

${ }^{296}$ Hamprecht, F.A., Cohen, A.J., Tozer, D.J., Handy, N.C. (1998) “Development and assessment of new exchange-correlation functionals", J. Chem. Phys. 109, 6264.

${ }^{297}$ Boese, A.D., Doltsinis, N.L., Handy, N.C., Sprik, M. (2000) “New generalized gradient approximation functionals", J. Chem. Phys. 112, 1670.

${ }^{298}$ Boese, A.D., Handy, N.C. (2001) “A new parametrization of exchange-correlation generalized gradient approximation functionals", J. Chem. Phys. 114, 5497.

${ }^{299}$ Becke, A.D. (1993) “Density-functional thermochemistry. III The role of exact exchange”, J. Chem. Phys. 98, 5648.

${ }^{300}$ Filatov, M., Shaik, S. (1998) "Spin-restricted density functional approach to the open-shell problem”, Chem. Phys. Lett. 288, 689.

${ }^{301}$ Filatov, M., Shaik, S. (1999) “Application of spin-restricted open-shell Kohn-Sham method to atomic and molecular multiplet states", J. Chem. Phys. 110, 116.

${ }^{302}$ Grimme, S. (1996) "Density functional calculations with configuration interaction for the excited states of molecules", Chem. Phys. Lett. 259, 128.

${ }^{303}$ See also Grimme, S., Waletzke, M. (1999) “A combination of Kohn-Sham density functional theory and multi-reference configuration interaction methods", J. Chem. Phys. 111, 5645 for an extension to multireference CI.

${ }^{304}$ Casida, M.E. (1995) “Time Dependent Density Functional Response Theory for Molecules”, In: Chong, D.P., ed, Recent Advances in Density Functional Methods, Part I (World Scientific, Singapore)

${ }^{305}$ Burke, K., Gross, E.K.U. (1998) “A Guided Tour of Time-Dependent Density Functional Theory”, In: Joubert, D., ed, Density Functionals: Theory and Applications, Lecture Notes in Physics, v. 500 (Springer, Heidelberg) 
${ }^{306}$ Petersilka, M., Gossmann, U.J., Gross, E.K.U. (1998) “Time Dependent Optimized Effective Potential in the Linear Response Regime”, In: Dobson, J.F., Vignale, G., Das, M.P., eds, Electronic Density Functional Theory, Recent Progress and New Directions (Plenum Press, New York)

${ }^{307}$ Huang, X., Lin, Z. (2003) “Density functional theory studies of ruthenium-catalyzed bis-Diels-Alder cycloaddition of 1,5-cyclooctadiene with alkynes", Organometallics 22, 5478.

${ }^{308}$ Khan, A. (2003) "Theoretical studies of $\mathrm{CO}_{2}\left(\mathrm{H}_{2} \mathrm{O}\right)_{20,24,28}$ clusters: stabilization of cages in hydrates by $\mathrm{CO}_{2}$ guest molecules", J. Mol. Struct. Theochem 664-665, 237.

${ }^{309}$ Thompson, D.J., Ciobîcă, I.M., Hodnett, B.K., van Santen, R.A., Fanning, M.O. (2003) “A DFT periodic study of the vanadyl pyrophosphate (100) surface", Surf. Sci. 547, 438.

${ }^{310}$ Pastore, G., Smargiassi, E., Buda, F. (1991) “Theory of ab initio molecular-dynamics calculations”, Phys. Rev. A 44, 6334.

${ }^{311}$ Remler, D.K., Madden, P.A. (1990) "Molecular dynamics without effective potentials via the CarParrinello approach”, Mol. Phys. 70, 921.

${ }^{312}$ Parrinello, M. (1997) "From silicon to RNA: The coming of age of ab initio molecular dynamics", Solid. State Comm. 102, 107.

${ }^{313}$ Tse, J.S. (2002) “Ab initio molecular dynamics with density functional theory”, Annu. Rev. Phys. Chem. 53, 249.

${ }^{314}$ Bolton, K, Hase, W.L., Peslherbe, G.H. (1998) “Mapping Multidimensional Intramolecular Dynamics Using Frequency Analysis”, In: Thompson, D.L., ed, Modern Methods for Multidimensional Dynamics Computations in Chemistry (World Scientific, Singapore)

${ }^{315}$ Car, R., Parrinello, M. (1985) "Unified approach for molecular dynamics and density-functional theory", Phys. Rev. Lett. 55, 2471.

316 There may be additional constraints imposed on the system, such as a fixed volume, pressure, etc. In this case there would be additional terms in the Lagrangian. Here we consider the simplest case, i.e. when only orthonormality constraints are imposed. For a more general treatment, see ref. 181, and also Laasonen, K., 
Pasquarello, A., Car, R., Lee, C., Vanderbilt, D. (1993) “Car-Parrinello molecular dynamics with Vanderbilt ultrasoft pseudopotentials", Phys. Rev. B 47, 10142.

${ }^{317}$ Verlet, L. (1967) “Computer "experiments” on classical fluids. I. Thermodynamical properties of Lennard-Jones molecules", Phys. Rev. 159, 98.

${ }^{318}$ Swope, W.C., Andersen, H.C., Berens, P.H., Wilson, K.R. (1982) “A computer simulation method for the calculation of equilibrium constants for the formation of physical clusters of molecules: Application to small water clusters", J. Chem. Phys. 76, 637.

319 Tuckerman, M., Berne, B.J., Martyna, G.J. (1992) “Reversible multiple time scale molecular dynamics”, J. Chem. Phys. 97, 1990.

${ }^{320}$ Tuckerman, M.E., Parrinello, M. (1994) “Integrating the Car-Parrinello equations. I. Basic integration techniques", J. Chem. Phys. 101, 1302.

${ }^{321}$ Tuckerman, M.E., Parrinello, M. (1994) “Integrating the Car-Parrinello equations. II. Multiple time scale techniques", J. Chem. Phys. 101, 1316.

${ }^{322}$ Hutter, J., Tuckerman, M.E., Parrinello, M. (1995) “Integrating the Car-Parrinello equations. III. Techniques for ultrasoft pseudopotentials", J. Chem. Phys. 102, 859.

${ }^{323}$ Nose, S. (1984) “A molecular dynamics method for simulations in the canonical ensemble", Mol Phys. 2, 255.

${ }^{324}$ Nose, S. (1984) “A unified formulation of the constant temperature molecular dynamics methods", $J$. Chem. Phys. 81, 511.

${ }^{325}$ Hoover, W.G. (1985) “Canonical dynamics: Equilibrium phase-space distributions”, Phys. Rev. A

31,1695 .

${ }^{326}$ Blöchl, P.E., Parrinello, M. (1992) “Adiabaticity in first-principles molecular dynamics”, Phys. Rev. B 45, 9413 (1992).

${ }^{327}$ Pasquarello, A., Laasonen, K., Car, R., Lee, Ch., Vanderbilt, D. (1992) “Ab initio molecular dynamics for d-electron systems: Liquid copper at 1500 K”, Phys. Rev. Lett. 69, 1982. 
${ }^{328}$ Ryckaert, J.P., Ciccotti, G., Berendsen, H.J.C. (1977) "Numerical integration of Cartesian equations of motion of a system with constraints: Molecular dynamics of n-alkanes”, J. Comput. Phys. 23, 237.

${ }^{329}$ Andersen, H.C. (1983) "RATTLE: A velocity version of the SHAKE algorithm for molecular dynamics calculations", J. Comput. Phys. 52, 24.

${ }^{330}$ See, for example, Giannozzi, P., Car, R., Scoles, G. (2003) “Oxygen adsorption on graphite and nanotubes", J. Chem. Phys. 118, 1003.

${ }^{331}$ Laio, A., Parrinello, M. (2002) “Escaping free-energy minima”, P. Natl. Acad. Sci. USA 99, 12562.

${ }^{332}$ Iannuzzi, M., Laio, A., Parrinello, M. (2003) “Efficient exploration of reactive potential energy surfaces using Car-Parrinello molecular dynamics”, Phys. Rev. Lett. 90, \#238302.

${ }^{333}$ Leforestier, V. (1978) "Classical trajectories using the full ab initio potential energy surface $\mathrm{H}^{-}$ $+\mathrm{CH}_{4} \rightarrow \mathrm{CH}_{4}+\mathrm{H}^{-”}$, J. Chem. Phys. 68, 4406.

${ }^{334}$ Field, M.J. (1991) "Constrained optimization of $a b$ initio and semiempirical Hartree-Fock wave functions using direct minimization or simulated annealing”, J. Phys. Chem. 95, 5104.

${ }^{335}$ Jellinek, J., Bonačić-Koutecký, V., Fantucci, P., Wiechert, M. (1994) “Ab initio Hartree-Fock selfconsistent-field molecular dynamics study of structure and dynamics of $\mathrm{Li}_{8}$ ", J. Chem. Phys. 101, 10092. ${ }^{336}$ Liu, Z., Carter, L.E., Carter, E.A. (1995) "Full configuration interaction molecular dynamics of $\mathrm{Na}_{2}$ and Na3”, J. Phys. Chem. 99, 4355.

${ }^{337}$ Senda, Y., Shimojo, F., Hoshino, K. (1999) “The origin of the first sharp diffraction peak in liquid Na-Pb alloys: ab initio molecular-dynamics simulations”, J. Phys.: Condens. Matter 11, 2199.

${ }^{338}$ Senda, Y., Shimojo, F., Hoshino, K. (1999) “Composition dependence of the structure and the electronic states of liquid K-Pb alloys: ab initio molecular-dynamics simulations”, J. Phys.: Condens. Matter 11, 5387 (1999)

${ }^{339}$ Ortega, J., Lewis, J.P., Sankey, O.F. (1994) “Simplified electronic-structure model for hydrogen-bonded systems: Water", Phys. Rev. B 50, 10516. 
${ }^{340}$ Ortega, J., Lewis, J.P., Sankey, O.F. (1997) “First principles simulations of fluid water: The radial distribution functions", J. Chem. Phys. 106, 3696.

${ }^{341}$ Gaigeot, M.-P., Sprik, M. (2003) “Ab initio molecular dynamics computation of the infrared spectrum of aqueous uracil", J. Phys. Chem. B 107, 10344.

${ }^{342}$ For a recent application to methanol, for example, see Pagliai, M., Cardini, G., Righini, R., Schettino, V. (2003) "Hydrogen bond dynamics in liquid methanol”, J. Chem. Phys. 119, 6655.

${ }^{343}$ Grossman, J.C., Schwegler, E., Draeger, E.W., Gygi, F., Galli, G. (2004) “Towards an assessment of the accuracy of density functional theory for first principles simulations of water", J. Chem. Phys. 120, 300.

${ }^{344}$ Trout, B.L., Parrinello, M. (1998) “The dissociation mechanism of $\mathrm{H}_{2} \mathrm{O}$ in water studied by firstprinciples molecular dynamics", Chem. Phys. Lett. 288, 343.

${ }^{345}$ Trout, B.L., Parrinello, M. (1999) “Analysis of the dissociation of $\mathrm{H}_{2} \mathrm{O}$ in water using first-principles molecular dynamics", J. Phys. Chem. B 103, 7340.

${ }^{346}$ Izvekov, S., Voth, G.A. (2002) “Car-Parrinello molecular dynamics simulation of liquid water: New results", J. Chem. Phys. 116, 10372.

${ }^{347}$ Buhl, M. (2002) "Structure, dynamics, and magnetic shieldings of permanganate ion in aqueous solution. A density functional study.”, J. Phys. Chem. A 106, 10505.

${ }^{348}$ Sillanpaa, A.J., Simon, C., Klein, M.L., Laasonen, K. (2002) “Structural and spectral properties of aqueous hydrogen fluoride studied using ab initio molecular dynamics", J. Phys. Chem. B 106, 11315. ${ }^{349}$ Röhrig, U.F., Frank, I., Hutter, J., Laio, A., VandeVondele, J., Rothlisberger, U. (2003) “QM/MM CarParrinello molecular dynamics study of the solvent effects on the ground state and on the first excited states of acetone in water", Chemphyschem 4, 1177.

${ }^{350}$ Doerksen, R.J., Chen, B., Klein, M.L. (2003) “Intramolecular hydrogen bonds: $a b$ initio Car-Parrinello simulations of arylamide torsions", Chem. Phys. Lett. 380, 150.

351 Åqvist, J., Warshel, A. (1993) "Simulation of enzyme reactions using valence bond force fields and other hybrid quantum/classical approaches", Chem. Rev. 93, 2523. 
${ }^{352}$ Gao, J. (1996) "Hybrid quantum and molecular mechanical simulations: An alternative avenue to solvent effects in organic chemistry", Acc. Chem. Res. 29, 298.

${ }^{353}$ Monard, G., Merz Jr., K.M. (1999) “Combined quantum mechanical/molecular mechanical methodologies applied to biomolecular systems", Acc. Chem. Res. 32, 904.

${ }^{354}$ Amara, P., Field, M.J. (1998) "Combined Quantum Mechanical and Molecular Mechanical Potentials", In: von Rague Schleyer, P., Allinger, N.L., Clark, T., Gasteiger, J., Kollman, P.A., Schaefer III, H.F., Schreiner, P.R., eds, Encyclopedia of Computational Chemistry (John Wiley \& Sons, Chichester) ${ }^{355}$ Ruiz-Lopez, M.F., Rivail, J.L. (1998) “Combined Quantum Mechanics and Molecular Mechanics Approaches to Chemical and Biochemical Reactivity", In: von Rague Schleyer, P., Allinger, N.L., Clark, T., Gasteiger, J., Kollman, P.A., Schaefer III, H.F., Schreiner, P.R., eds, Encyclopedia of Computational Chemistry (John Wiley \& Sons, Chichester)

${ }^{356}$ Merz Jr., K.M., Stanton, R.V. (1998) “Quantum Mechanical/Molecular Mechanical (QM/MM) Coupled Potentials”, In: von Rague Schleyer, P., Allinger, N.L., Clark, T., Gasteiger, J., Kollman, P.A., Schaefer III, H.F., Schreiner, P.R., eds, Encyclopedia of Computational Chemistry (John Wiley \& Sons, Chichester) ${ }^{357}$ Tomasi, J., Pomelli, C.S. (1998) “Quantum Mechanics/Molecular Mechanics”, In: von Rague Schleyer, P., Allinger, N.L., Clark, T., Gasteiger, J., Kollman, P.A., Schaefer III, H.F., Schreiner, P.R., eds, Encyclopedia of Computational Chemistry (John Wiley \& Sons, Chichester)

${ }^{358}$ Sauer, J., Sierka, M. (2000) "Combining quantum mechanics and interatomic potential functions in $a b$ initio studies of extended systems", J. Comput. Chem. 21, 1470

${ }^{359}$ Warshel, A. (1997) Computer Modeling of Chemical Reactions in Enzymes and Solutions (Wiley, New York)

${ }^{360}$ Clementi, E. (1980) Computational Aspects for Large Chemical Systems (Springer-Verlag, Berlin)

${ }^{361}$ Gao, J., Thompson M.A.,eds (1998) Combined Quantum Mechanical and Molecular Mechanical Methods (American Chemical Society, Washington) 
${ }^{362}$ Tomasi, J., Persico, M. (1994) "Molecular interactions in solution: An overview of methods based on continuous distributions of the solvent”, Chem. Rev. 94, 2027.

${ }^{363}$ Hwang, J.-K., King, G., Creighton, S., Warshel, A. (1988) "Simulation of the free energy relationships and dynamics of $\mathrm{S}_{\mathrm{N}} 2$ reactions in aqueous solution", J. Am. Chem. Soc. 110, 5297.

${ }^{364}$ Wesolowski, T.A., Warshel, A. (1993) "Frozen density functional approach for $a b$ initio calculations of solvated molecules", J. Phys. Chem. 97, 8050.

${ }^{365}$ Stanton, R.V., Hartsough, D.S., Merz Jr., K.M. (1993) “Calculation of solvation free energies using a density functional/molecular dynamics coupled potential”, J. Phys. Chem. 97, 11868.

${ }^{366}$ Gao, J., Truhlar, D.G. (2002) “Quantum mechanical methods for enzyme kinetics”, Annu. Rev. Phys. Chem. 53, 467.

${ }^{367}$ Truhlar, D.G., Gao, J.L., Alhambra, C., García-Viloca, M., Corchado, J., Sanchez, M.L., Villa, J. (2002) “The incorporation of quantum effects in enzyme kinetics modeling”, Acc. Chem. Res. 35, 341.

${ }^{368}$ Warshel, A., Levitt, M. (1976) “Theoretical studies of enzymic reactions: Dielectric, electrostatic and steric stabilization of carbonium ion in reaction of lysozyme", J. Mol. Biol. 103, 227.

${ }^{369}$ Field, M.J., Bash, P.A., Karplus, M. (1990) “A combined quantum mechanical and molecular mechanical potential for molecular dynamics simulations", J. Comput. Chem. 11, 700.

${ }^{370}$ Very often the method of choice for the quantum mechanical part is DFT due to its good accuracy/cost ratio.

${ }^{371}$ An additional term including the contribution from the boundary region, $H_{\text {boundary }}$ can also be included.

${ }^{372}$ Svensson, M., Humbel, S., Froese, R.D.J., Matsubara, T., Sieber, S., Morokuma, K. (1996) “ONIOM: A multilayered integrated $\mathrm{MO}+\mathrm{MM}$ method for geometry optimizations and single point energy predictions. A test for Diels-Alder reactions and $\operatorname{Pt}\left(\mathrm{P}(t-\mathrm{Bu})_{3}\right)_{2}+\mathrm{H}_{2}$ oxidative addition”, J. Phys. Chem. 100, 19357.

${ }^{373}$ Froese, R.D.J., Morokuma, K. (1998) “The ONIOM Method. Integration of Different Levels of Molecular Orbital Methods and/or Molecular Mechanics Methods for Large Molecular Systems and Its Applications to Structures, Energies and Chemical Reactions”, In: von Rague Schleyer, P., Allinger, N.L., Clark, T., 
Gasteiger, J. , Kollman, P.A., Schaefer III, H.F., Schreiner, P.R., eds, Encyclopedia of Computational Chemistry (John Wiley \& Sons, Chichester)

${ }^{374}$ Sherwood, P., de Vries, A.H., Guest, M. F., Schreckenbach, G., Catlow, C.R.A., French, S.A., Sokol, A.A., Bromley, S.T., Thiel, W., Turner, A.J., Billeter, S., Terstegen, F., Thiel, S., Kendrick, J., Rogers, S.C., Casci, J., Watson, M., King, F., Karlsen, E., Sjøvoll, M., Fahmi, A., Schäfer, A., Lennartz, C. (2003)

"QUASI: A general purpose implementation of the QM/MM approach and its application to problems in catalysis", J. Mol. Struct. Theochem 632, 1.

${ }^{375}$ Warshel, A., Weiss, R.M. (1980) “An empirical valence bond approach for comparing reactions in solutions and in enzymes", J. Am. Chem. Soc. 102, 6218.

${ }^{376}$ Li, G., Cui, Q. (2003) "What is so special about Arg 55 in the catalysis of Cyclophilin A? Insights from hybrid QM/MM simulations", J. Am. Chem. Soc. 125, 15028.

${ }^{377}$ Shigeta, Y. (2004) "Hybrid QM/MM studies on energetics of malonaldehyde in condensed phase", Int. J. Quant. Chem. 96, 32.

${ }^{378}$ Hill, T.L. (1960) An Introduction to Statistical Thermodynamics (Dover, New York)

${ }^{379}$ Gray, C.C., Gubbins, K.E. (1984) Theory of Molecular Fluids (Clarendon Press, Oxford)

${ }^{380}$ This is not the only possibility. In particular, when simulating a chemical reaction at very high temperatures, there is a coupling of the internal degrees of freedom of the molecule and the molecular partition function cannot be factored as in (2.5.3). We will not concern ourselves with such complications here.

${ }^{381}$ Coker, D.F., Watts, R.O. (1981) “Computer simulation of reactive liquids in chemical equilibrium”, Chem. Phys. Lett. 78, 333

${ }^{382}$ Coker, D.F., Watts, R.O. (1981) "Chemical equilibria in mixtures of bromine and chlorine”, Mol. Phys. 44, 1303.

${ }^{383}$ Norman, G.E., Filinov, V.S. (1969) “Investigations of phase transitions by a Monte Carlo method”, High Temp. 7, 216. 
${ }^{384}$ Adams, D.J. (1974) “Chemical potential of hard-sphere fluids by Monte Carlo methods", Mol. Phys. 28, 1241

${ }^{385}$ Adams, D.J. (1975) “Grand canonical ensemble Monte Carlo for a Lennard-Jones fluid”, Mol. Phys. 29, 307.

${ }^{386}$ Kofke D.A., Glandt, E.D. (1988) "Monte Carlo simulation of multicomponent equilibria in a semigrand canonical ensemble", Mol. Phys. 64, 1105.

${ }^{387}$ Shaw M.S. (1991) "Monte Carlo simulation of equilibrium chemical composition of molecular fluid mixtures in the $\mathrm{N}_{\text {atoms }} \mathrm{PT}$ ensemble", J. Chem. Phys. 94, 7550.

${ }^{388}$ Busch, N.A., Wertheim, W.S., Chiew, Y.C., Yarmush, M.L. (1994) “A Monte Carlo method for simulating associating fluids", J. Chem. Phys. 101, 3147.

${ }^{389}$ Busch, N.A., Wertheim, M.S., Yarmush, M.L. (1996) “Monte Carlo simulation of $n$-member associating fluids: Application to antigen-antibody systems", J. Chem. Phys. 104, 3962.

${ }^{390}$ Tsangaris, D.M., de Pablo, J.J. (1994) "Bond-bias simulation of phase equilibria for strongly associated fluids", J. Chem. Phys. 101, 1477.

${ }^{391}$ Shew, C.-Y., Mills, P. (1993) “A Monte Carlo method to simulate systems with barriers: subspace sampling", J. Phys. Chem. 97, 13824.

${ }^{392}$ Shew, C.-Y., Mills, P. (1995) “The subspace sampling method: A Monte Carlo approach for simulating the single-particle density function and the equilibrium constant for systems described by multiple Hamiltonians", J. Phys. Chem. 99, 12980.

${ }^{393}$ Shew, C.-Y., Mills, P. (1995) "Monte Carlo simulations of the pair correlation function and the equilibrium association constant of the sticky electrolyte model using the subspace sampling method", $J$. Phys. Chem. 99, 12988.

${ }^{394}$ Turner, C.H. (2002), PhD Thesis (North Carolina State University, Raleigh)

${ }^{395}$ Smith, W.R., Triska, B. (1994) "The reaction ensemble method for the computer simulation of chemical and phase equilibria. I. Theory and basic examples", J. Chem. Phys. 100, 3019. 
${ }^{396}$ Johnson, J.K., Panagiotopoulos, A.Z., Gubbins, K.E. (1994) "Reactive canonical Monte Carlo: A new simulation technique for reacting or associating fluids", Mol. Phys. 81, 717.

${ }^{397}$ Johnson, J.K. (1991) "Reactive canonical Monte Carlo", Adv. Chem. Phys. 105, 461.

${ }^{398}$ Turner, C.H., Johnson, J.K., Gubbins, K.E. (2001) “Effect of confinement on chemical reaction equilibria: The reactions $2 \mathrm{NO} \leftrightarrow(\mathrm{NO})_{2}$ and $\mathrm{N}_{2}+3 \mathrm{H}_{2} \leftrightarrow 2 \mathrm{NH}_{3}$ in carbon micropores", J. Chem. Phys. 114, 1851 .

${ }^{399}$ Turner, C.H., Pikunic, J., Gubbins, K.E. (2001) “Influence of chemical and physical surface heterogeneity on chemical reaction equilibria in carbon micropores", Mol. Phys. 99, 1991.

${ }^{400}$ Turner, C.H., Brennan, J.K., Johnson, J.K., Gubbins, K.E. (2002) “Effect of confinement by porous materials on chemical reaction kinetics", J. Chem. Phys. 116, 2138.

${ }^{401}$ Turner, C.H., Brennan, J.K., Pikunic, J. (2002) "Simulation of chemical reaction equilibria and kinetics in heterogeneous carbon micropores", Appl. Surf. Sci. 196, 366.

${ }^{402}$ Turner, C.H., Gubbins, K.E. (2003) "Effects of supercritical clustering and selective confinement on reaction equilibrium: A molecular simulation study of the esterification reaction”, J. Chem. Phys. 119, 6057 ${ }^{403}$ Lisal, M., Nezbeda, I., Smith, W.R. (1999) "The reaction ensemble method for the computer simulation of chemical and phase equilibria. II. The $\mathrm{Br}_{2}+\mathrm{Cl}_{2}+\mathrm{BrCl}$ system”, Journal of Chemical Physics 110, 8597 ${ }^{404}$ Smith, W.R., Lisal, M. (2002) “Direct Monte Carlo simulation methods for nonreacting and reacting systems at fixed total internal energy or enthalpy”, Phys. Rev. E 66, \#011104.

${ }^{405}$ Lisal, M., Smith, W.R., Nezbeda, I. (2000) “Computer simulation of the thermodynamic properties of high-temperature chemically-reacting plasmas", J. Chem. Phys. 113, 4885.

${ }^{406}$ Lisal, M., Smith, W.R., Nezbeda, I. (1999) “Accurate computer simulation of phase equilibrium for complex fluid mixtures. Application to binaries involving isobutene, methanol, methyl tert-butyl ether, and n-butane”, J. Phys. Chem. B 103, 10496.

${ }^{407}$ Lisal, M., Smith, W.R., Nezbeda, I. (2000) “Accurate vapour-liquid equilibrium calculations for complex systems using the reaction Gibbs ensemble Monte Carlo simulation method", Fluid Phase Equil. 181, 127. 
${ }^{408}$ de Souza, L.E.S., Deiters, U.K. (1999) "Non-ideality of the system $\mathrm{NH}_{3}-\mathrm{H}_{2}-\mathrm{N}_{2}$. Comparison of equation of state and simulation predictions with experimental data", Phys. Chem. Chem. Phys. 1, 4069.

${ }^{409}$ Borówko, M., Zagórski, R. (2001) “Chemical equilibria in slitlike pores”, J. Chem. Phys. 114, 5397.

${ }^{410}$ Brennan, J.K., Rice, B.M. (2002) "Molecular simulation of shocked materials using the reactive Monte Carlo method", Phys. Rev. E 66, \#021105.

${ }^{411}$ Truhlar, D.G., Hase, W.L., Hynes, J.T. (1983) “Current status of transition-state theory”, J. Phys. Chem. 87, 2664.

${ }^{412}$ Truhlar, D.G., Garrett, B.C., Klippenstein, S.J. (1996) “Current status of transition-state theory”, J. Phys. Chem. 100, 12771.

${ }^{413}$ Garrett B. C., Truhlar, D. G. (1998) “Transition State Theory”, In: Schleyer, P. v. R., Allinger, N. L., Clark, T., Gasteiger, J., Kollman, P. A., Schaefer III, H. F.,eds, Encyclopedia of Computational Chemistry (John Wiley, Chichester)

${ }^{414}$ Eyring, H. (1935) “Activated complex in chemical reactions”, J. Chem. Phys. 3, 107.

${ }^{415}$ Petersson, G.A. (2000) "Perspective on "The activated complex in chemical reactions" Eyring H (1935) J Chem Phys 3: 107”, Theor. Chem. Acc. 103, 190.

${ }^{416}$ Yamamoto, T. (1960) "Quantum statistical mechanical theory of the rate of exchange chemical reactions in the gas phase", J. Chem. Phys. 33, 281.

${ }^{417}$ See, for example, refs. 11,420

${ }^{418}$ This expression is ensemble-independent, see e.g. ref. 420

${ }^{419}$ Bennett, C.H. (1977) “Algorithms for Chemical Computations”, In: Christofferson, R.E., ed, ACS

Symposium Series v. 46, (American Chemical Society, Washington)

${ }^{420}$ Chandler, D. (1978) "Statistical mechanics of isomerization dynamics in liquids and the transition state approximation”, J. Chem. Phys. 68, 2959.

${ }^{421}$ Chandler, D. (1987) Introduction to Modern Statistical Mechanics (Oxford University Press, Oxford) 
${ }^{422}$ Chandler, D. (1998) "Barrier Crossings: Classical Theory of Rare but Important Events”, In: Berne, B.J., Ciccotti, G., Coker, D.F.,eds, Computer Simulation of Rare Events and Dynamics of Classical and Quantum Condensed-Phase Systems - Classical and Quantum Dynamics in Condensed Phase Simulations (World Scientific, Singapore)

${ }^{423}$ Mahan, B.H. (1974) “Activated complex theory of bimolecular reactions”, J. Chem. Educ. 51, 709.

${ }^{424}$ Miller, W.H. (1976) "Importance of nonseparability in quantum mechanical transition-state theory", Acc. Chem. Res. 9, 306.

${ }^{425}$ Truhlar, D.G., Garrett, B.C. (1980) "Variational transition-state theory”, Acc. Chem. Res. 13, 440.

${ }^{426}$ Miller, W.H., Schwartz, S.D., Tromp, J.W. (1983) “Quantum mechanical rate constants for bimolecular reactions", J. Chem. Phys. 79, 4889.

${ }^{427}$ Tromp, J.W., Miller, W.H. (1986) "New approach to quantum mechanical transition-state theory", J. Phys. Chem. 90, 3482.

${ }^{428}$ Tromp, J.W., Miller, W.H. (1987) “The reactive flux correlation function for collinear reactions $\mathrm{H}+\mathrm{H}_{2}$, $\mathrm{Cl}+\mathrm{HCl}$ and $\mathrm{F}+\mathrm{H}_{2} "$, Faraday Discuss. Chem. Soc. 84, 441.

${ }^{429}$ Day, P.N., Truhlar, D.G. (1991) "Calculation of thermal rate coefficients from the quantum flux autocorrelation function: Converged results and variational quantum transition state theory for $\mathrm{O}+\mathrm{HD} \leftrightarrow \mathrm{OD}+\mathrm{H}$ and $\mathrm{O}+\mathrm{HD} \leftrightarrow \mathrm{OH}+\mathrm{D}$ ", J. Chem. Phys. 95, 5097.

${ }^{430}$ Voth, G.A., Chandler, D., Miller, W.H. (1989) “Rigorous formulation of quantum transition state theory and its dynamical corrections", J. Chem. Phys. 91, 7749.

${ }^{431}$ Voth, G.A. (1993) "Feynman path integral formulation of quantum mechanical transition-state theory", $J$. Phys. Chem. 97, 8365.

${ }^{432}$ Stuchebrukhov, A.A. (1991) “Green's functions in quantum transition state theory”, J. Chem. Phys. 95, 4258.

${ }^{433}$ Mills, G., Schenter, G.K., Makarov, D.E., Jónsson, H. (1998) "RAW Quantum Transition State Theory”, In: Berne, B.J., Ciccotti, G., Coker, D.F., eds, Computer Simulation of Rare Events and Dynamics of 
Classical and Quantum Condensed-Phase Systems - Classical and Quantum Dynamics in Condensed Phase Simulations, (World Scientific, Singapore)

${ }^{434}$ Mills, G., Schenter, G.K., Makarov, D., Jónsson, H. (1997) “Generalized path integral based quantum transition state theory", Chem, Phys. Lett. 278, 91.

${ }^{435}$ Hansen, N.F., Anderson, H.C. (1994) “A new formulation of quantum transition state theory for adiabatic rate constants", J. Chem. Phys. 101, 6032.

${ }^{436}$ Cheney, B.G., Andersen, H.C. (2003) "Dynamical corrections to quantum transition state theory”, $J$. Chem. Phys. 118, 9542.

${ }^{437}$ Park, T.J., Light, J.C. (1986) “Unitary quantum time evolution by iterative Lanczos reduction”, J. Chem. Phys. 85, 5870.

${ }^{438}$ Miller, W.H. (1993) "Beyond transition-state theory: a rigorous quantum theory of chemical reaction rates", Acc. Chem. Res. 26, 174.

${ }^{439}$ Manthe, U., Seideman, T., Miller, W.H. (1993) "Full-dimensional quantum mechanical calculation of the rate constant for the $\mathrm{H}_{2}+\mathrm{OH} \rightarrow \mathrm{H}_{2} \mathrm{O}+\mathrm{H}$ reaction", J. Chem. Phys. 99, 10078.

${ }^{440}$ Barik, D., Banik, S.K., Ray, D.S. (2003) “Quantum phase-space function formulation of reactive flux theory", J. Chem. Phys. 119, 680.

${ }^{441}$ For a reactive-flux formulation for mixed quantum/classical dynamics, see Sergi, A., Kapral, R. (2003) "Quantum-classical dynamics of nonadiabatic chemical reactions", J. Chem. Phys. 118, 8566 and references therein.

${ }^{442}$ Miller, W.H. (1998) ““'Direct” and "correct” calculation of canonical and microcanonical rate constants for chemical reactions", J. Phys. Chem. A, 102, 793.

${ }^{443}$ Miller, W. H. (1998) "Spiers memorial lecture quantum and semiclassical theory of chemical reaction rates", Faraday Discuss. 110, 1.

${ }^{444}$ See, for example, Schenter, G.K., Kathmann, S.M., Garrett, B.C. (1999) "Variational transition state theory of vapor phase nucleation", J. Chem. Phys. 110, 7951. 
${ }^{445}$ See, for example, Bader, J.S., Berne, B.J., Pollak, E. (1995) “Activated rate processes: The reactive flux method for one-dimensional surface diffusion”, J. Chem. Phys. 102, 4037.

${ }^{446}$ See, for example, Voter, A.F. (1997) "A method for accelerating the molecular dynamics simulation of infrequent events", J. Chem. Phys. 106, 4665.

${ }^{447}$ Drozdov, A.N., Tucker, S.C. (2001) “An improved reactive flux method for evaluation of rate constants in dissipative systems", J. Chem. Phys. 115, 9675.

${ }^{448}$ Ocassionally, a full moon occurs twice in a given calendar month. The second occurred is referred to as a "Blue Moon".

${ }^{449}$ Carter, E.A., Ciccotti, G., Hynes, J.T., Kapral, R. (1989) “Constrained reaction coordinate dynamics for the simulation of rare events", Chem. Phys. Let. 156, 472.

${ }^{450}$ Ciccotti, G., Ferrario, M. (2000) “Rare events by constrained molecular dynamics”, J. Molec. Liquids 89, 1 .

${ }^{451}$ Coluzza, I., Sprik, M., Ciccotti, G. (2003) "Constrained reaction coordinate dynamics for systems with constraints", Mol. Phys. 101, 2885.

${ }^{452}$ Sprik, M., Ciccotti, G. (1998) "Free energy from constrained molecular dynamics”, J. Chem. Phys. 109, 7737.

${ }^{453}$ Paci, E., Ciccotti, G., Ferrario, M., Kapral, R. (1991) “Activation energies by molecular dynamics with constraints", Chem. Phys. Lett. 176, 581.

${ }^{454}$ A holonomic constraint is a constraint that involves only configurational variables, not momenta.

${ }^{455}$ This, of course, assumes that there is a way to obtain atom-atom interactions that is not too computationally costly, such as a reactive force field. If the potentials are obtained from "on-the-fly" $a b$ initio calculations, as in CPMD, the system sizes that can be handled by the method are much smaller. ${ }^{456}$ Meijer, E.J., Sprik, M. (1998) “Ab initio molecular dynamics study of the reaction of water with formaldehyde in sulfuric acid solution", J. Am. Chem. Soc. 120, 6345. 
${ }^{457}$ Ensing, B., Meijer, E.J., Blöchl, B.E., Baerends, E.J. (2001) "Solvation effects on the $\mathrm{S}_{\mathrm{N}} 2$ reaction between $\mathrm{CH}_{3} \mathrm{Cl}$ and $\mathrm{Cl}^{-}$in water", J. Phys. Chem. A 105, 3300 .

${ }^{458}$ Pagliai, M., Raugei, S., Cardini, G., Schettino, V. (2003) "Intramolecular solvation effects in the $\mathrm{S}_{\mathrm{N}} 2$ reaction $\mathrm{Cl}^{-}+\mathrm{Cl}\left(\mathrm{CH}_{2}\right)_{\mathrm{n}} \mathrm{CN}$ ", J. Chem. Phys. 119, 9063.

${ }^{459}$ Mugnai, M., Cardini, G., Schettino, V. (2003) “An ab initio molecular dynamics study of the $\mathrm{S}_{\mathrm{N}} 2$ reaction $\mathrm{F}^{-}+\mathrm{CH}_{3} \mathrm{Cl} \rightarrow \mathrm{CH}_{3} \mathrm{~F}+\mathrm{Cl}^{-’}$, J. Chem. Phys. 118, 2767.

${ }^{460}$ Mugnai, M., Cardini, G., Schettino, V. (2003) "Substitution and elimination reaction of $\mathrm{F}^{-}$with $\mathrm{C}_{2} \mathrm{H}_{5} \mathrm{Cl}$ : An $a b$ initio molecular dynamics study", J. Phys. Chem. A 107, 2540.

${ }^{461}$ Doltsinis, N., Sprik, M. (2003) “Theoretical $\mathrm{pK}_{\mathrm{a}}$ estimates for solvated $\mathrm{P}(\mathrm{OH})_{5}$ from coordination constrained Car-Parrinello molecular dynamics", Phys. Chem. Chem. Phys. 5, 2612.

${ }^{462}$ Dellago, C., Bolhuis, P.G., Csajka, F.S., Chandler, D. (1998) “Transition path sampling and the calculation of rate constants", J. Chem. Phys. 108, 1964.

${ }^{463}$ Dellago, C., Bolhuis, P.G., Chandler, D. (1999) “On the calculation of reaction rate constants in the transition path ensemble", J. Chem. Phys. 110, 6617.

${ }^{464}$ Dellago, C., Bolhuis, P.G., Chandler, D. (1998) "Efficient transition path sampling: Application to Lennard-Jones cluster rearrangements", J. Chem. Phys. 108, 9236.

${ }^{465}$ Bolhuis, P.G., Dellago, C., Chandler, D. (1998) "Sampling ensembles of deterministic transition pathways", Faraday Discuss. Chem. Soc. 110, 421.

${ }^{466}$ Bolhuis, P.G., Chandler, D., Dellago, C., Geissler, P.L. (2002) “Transition path sampling: Throwing ropes over rough mountain passes, in the dark", Annu. Rev. Phys. Chem. 53, 291.

${ }^{467}$ Chandler, D. (1998) "Finding Transition Pathways: Throwing Ropes Over Rough Mountain Passes, in the Dark”, In: Berne, B.J., Ciccotti, G., Coker, D.F., eds, Computer Simulation of Rare Events and Dynamics of Classical and Quantum Condensed-Phase Systems - Classical and Quantum Dynamics in Condensed Phase Simulations (World Scientific, Singapore)

${ }^{468}$ Dellago, C., Bolhuis, P.G., Geissler, P.L. (2002) “Transition path sampling”, Adv. Chem. Phys. 123, 1. 
${ }^{469}$ Passerone, D., Parrinello, M. (2001) “Action-derived molecular dynamics in the study of rare events”, Phys. Rev. Lett. 87, \#108302.

${ }^{470}$ van Erp, T.S., Moroni, D., Bolhuis, P.G. (2003) "A novel path sampling method for the calculation of rate constants", J. Chem. Phys. 118, 7762.

${ }^{471}$ Ramírez, J., Laso, M. (2001) “Conformational kinetics in liquid $n$-butane by transition path sampling”, $J$. Chem. Phys. 115, 7285.

${ }^{472}$ Bolhuis, P.G., Dellago, C., Chandler, D. (2000) "Reaction coordinates of biomolecular isomerization", Proc. Nat. Acad. Sci. U.S., 97, 5877.

${ }^{473}$ Bolhuis, P.G., Chandler, D. (2000) “Transition path sampling of cavitation between molecular scale solvophobic surfaces", J. Chem. Phys. 113, 8154.

${ }^{474}$ Geissler, P.L., Dellago, C., Chandler, D. (1999) "Kinetic pathways of ion pair dissociation in water", J. Phys. Chem. B 103, 3706.

${ }^{475}$ Geissler, P.L., Dellago, C., Chandler, D., Hutter, J., Parrinello, M. (2001), “Autoionization in liquid water", Science 291, 2121.

${ }^{476}$ Ensing, B., Baerends, E.J. (2002) "Reaction path sampling of the reaction between iron(II) and hydrogen peroxide in aqueous solution", J. Phys. Chem. A 106, 7902.

${ }^{477}$ Bolhuis, P.G. (2003) “Transition path sampling of $\beta$-hairpin folding”, Proc. Nat. Acad. Sci. USA 100, 12129.

${ }^{478}$ Hagan, M.F., Dinner, A.R., Chandler, D., Chakraborty, A.K. (2003) “Atomistic understanding of kinetic pathways for single base-pair binding and unbinding in DNA", Proc. Nat. Acad. Sci. U.S., 100, 13922. 


\section{Chapter 3}

\section{Isomerization of Small Hydrocarbons in Narrow Slit Pores and the Double Exponential Effect}

\subsection{Introduction}

As was mentioned in the introductory chapter, the main goal of this project is to obtain a fundamental understanding of each of the different types of effects that confinement can have on different chemical reactions. One of the most interesting and little-understood effects of confinement is what we term the "shape-catalytic" effect, i.e. the influence that the geometry of the confining material can have on both the equilibrium and the dynamics of a chemical reaction.

As part of an effort to understand this shape-catalytic effect at a fundamental level, we have carried out density functional theory calculations on the rotational isomerization of three 4-membered hydrocarbons: n-butane, 1-butene and 1,3-butadiene. Our results show that the interactions with the carbon walls cause a dramatic change on the potential energy surface for pore sizes comparable to the molecular dimensions. The porous material enhances or hinders reactions depending on how similar the shape of the transition state is to the shape of the confining material. The structure of the stable states and their equilibrium distributions are also drastically modified by confinement. Our results are consistent with a doubly exponential behavior of the reaction rates as a function of pore size, illustrating how the shape of a catalytic support can dramatically change the efficiency of a catalyst. 
The rest of this chapter is organized as follows: in section 3.2 we describe the methods used to obtain the torsional energy profiles of the three hydrocarbons studied, and the methods used to estimate the rates of isomerization in the gas phase and in confinement. In section 3.3 we summarize our findings on the effect of confinement on the energetics and the dynamics of all three systems, and provide a simple theoretical explanation for the observed double-exponential effect on the reaction rates. Finally, in section 3.4 we make some concluding remarks and discuss the possibility of integrating this kind of shapecatalytic effect in the design of highly effective catalytic materials. The material from this chapter has been published in refs. 1-6.

\subsection{Methods}

The results presented in this chapter were obtained using plane-wave pseudopotential density functional theory ${ }^{7,8}$ (DFT), with the Becke-Lee-Yang-Parr (BLYP) exchangecorrelation functional ${ }^{9,10}$. We used ultrasoft pseudopotentials ${ }^{11}$ with a plane-wave cutoff of 35 Ry and a density cutoff of 280 Ry. The calculations were done using the CPMD package $^{12}$.

We found the torsional energy profile for n-butane, 1-butene and 1,3-butadiene in two different environments: (1) In the gas phase and (2) between two graphene sheets separated by distances between 11 a.u. (5.82 $\AA$ ) and 15 a.u. (7.94 $\AA$ ). The first set of calculations serves as a comparison point to gauge the accuracy of our results. The second set of calculations represents the rotational isomerizations happening in carbon pores of sizes comparable to the molecular size. We also carried out calculations for the torsional profile of n-butane physisorbed on a single graphene sheet using an empirical van der Waals correction to the DFT energies ${ }^{13-15}$, but did not find any appreciable effect on the torsional 
profile. This shows that the effect of confinement is mostly due to the steric hindrance imposed by the pore walls.

The torsional energy profile for all three hydrocarbons was obtained using the following procedure: First, we performed a series of calculations with the dihedral angle between the four carbon atoms constrained to values ranging from zero to 180 degrees in steps of 15 degrees (5 degrees for the ideal gas n-butane calculations). Then we relaxed the stable states, and optimized the transition state structures using the partitioned rational function optimization (P-RFO) method ${ }^{16,17}$. In some cases, especially for the smaller pore sizes, we repeated the calculations with different starting positions within the pore, and carried out more constrained geometry optimizations around the transition state regions. This was necessary because, for the smaller pore sizes, the detailed structure of the pore walls becomes important and it is possible for the confined molecule to be trapped at metastable sites within the pore.

The graphene walls were modeled as 32-atom sheets on a hexagonal unit cell with periodic boundary conditions. The cell dimensions were allowed to relax fully before the calculation, yielding an equilibrium carbon-carbon bond length of $1.43 \AA$. In order to maintain a constant pore size in the nanopore calculations, we constrained the atoms on the graphene sheets to remain separated by the desired pore width.

In order to illustrate the effect of confinement on the isomerization kinetics, we calculated the variational transition state theory (VTST) rate constant ${ }^{18}$ in the ideal gas limit (Henry's law limit on the pores) for the all the asymmetric transitions (anti-gauche (syn) for nbutane, syn-skew (anti) for 1-butene, s-trans-gauche (s-cis) for 1,3-butadiene) for all the pore sizes considered. In order to obtain these values, we ran phonon calculations for the anti, gauche, and eclipsed (transition state) conformations, and calculated the corresponding ideal-gas partition functions using the ParFuMS code ${ }^{19-21}$. For the adsorbed 
states, the translational and rotational modes were treated as those of a 2-dimensional ideal gas. In all cases, we treated rotations, translations and vibrations as independent of each other. For the 'activated' (i.e. nonequilibrium) conformations, we projected out the reactive mode as obtained from the equation defining the torsional constraint ${ }^{22-24}$. The partition function was then used to obtain the free energy as a function of temperature at all conformations and pore widths in the ideal gas limit (in the bulk) or Henry's law limit (in the pores). We then splined the data at each temperature as a function of the torsion angle, located the maximum of the activation free energy barrier, and used it to calculate the VTST rate constant.

\subsection{Results and Discussion}

In order to clarify the discussion in the following sections, we present in table $3.1 \mathrm{a}$ summary of the stable conformers of n-butane, 1-butene and 1,3-butadiene both in the bulk and within carbon slit pores. Figure 3.1 also shows snapshots of the various molecular conformations to aid in visualization. A more detailed description of the torsional profiles is given in the next subsections.

For n-butane, the global minimum both in the bulk and within the pores is an anti conformer, with the methyl end groups pointing away from each other at $180^{\circ}$. The geometry of the high-energy conformer, however, changes with decreasing pore width because the steric hindrance imposed by the pore walls favors the planar conformations. Thus, the gauche conformation at $65.0^{\circ}$ is first changed to a more planar gauche conformation at $58.2^{\circ}$ within the pore of width 15 a.u., then to an even more planar conformation at $38.9^{\circ}$ within the 14 a.u.-pore, and below this pore width the syn conformer, at $0^{\circ}$, becomes stable. 
For 1-butene, the global energy minimum in the bulk is a skew conformer, with a C-C-C-C dihedral angle of $121.2^{\circ}$. As is the case with the gauche conformer of n-butane, this nonplanar conformation interacts strongly with the pore walls and the torsion angle within the pore drifts toward the planar anti conformation at $180^{\circ}$ as pore size decreases. The highenergy conformer in the bulk, with a syn geometry $\left(0^{\circ}\right)$, actually becomes the energy minimum within the pores, as it has a lower steric hindrance between the methyl and methylene end groups than the distorted skew and anti conformers.

Table 3.1. Stable conformers of n-Butane, 1-Butene, and 1,3-Butadiene in the bulk and within carbon slit pores. The $\mathrm{C}-\mathrm{C}-\mathrm{C}-\mathrm{C}$ dihedral angle for each conformer is given in parenthesis below its name.

\begin{tabular}{ccccccc}
\hline Molecule $\rightarrow$ & \multicolumn{2}{c}{ n-Butane } & \multicolumn{2}{c}{ 1-Butene } & \multicolumn{2}{c}{ 1,3-Butadiene } \\
\hline Pore Width $\downarrow$ & $\begin{array}{c}\text { Global } \\
\text { minimum }\end{array}$ & $\begin{array}{c}\text { High- } \\
\text { energy } \\
\text { conformer }\end{array}$ & $\begin{array}{c}\text { Global } \\
\text { minimum }\end{array}$ & $\begin{array}{c}\text { High- } \\
\text { energy } \\
\text { conformer }\end{array}$ & $\begin{array}{c}\text { Global } \\
\text { minimum }\end{array}$ & $\begin{array}{c}\text { High- } \\
\text { energy } \\
\text { conformer }\end{array}$ \\
\hline Bulk $(\infty)$ & $\begin{array}{c}\text { anti } \\
\left(180^{\circ}\right)\end{array}$ & $\begin{array}{c}\text { gauche } \\
\left(65.0^{\circ}\right)\end{array}$ & $\begin{array}{c}\text { skew } \\
\left(121.2^{\circ}\right)\end{array}$ & $\begin{array}{c}\text { syn } \\
\left(0^{\circ}\right)\end{array}$ & $\begin{array}{c}\text { s-trans } \\
\left(180^{\circ}\right)\end{array}$ & $\begin{array}{c}\text { gauche } \\
\left(30.3^{\circ}\right)\end{array}$ \\
15 a.u. & anti & gauche & syn & skew & s-trans & gauche \\
$(7.9 \AA)$ & $\left(180^{\circ}\right)$ & $\left(58.2^{\circ}\right)$ & $\left(0^{\circ}\right)$ & $\left(135.0^{\circ}\right)$ & $\left(180^{\circ}\right)$ & $\left(26.7^{\circ}\right)$ \\
14 a.u. & anti & gauche & syn & skew & s-trans & gauche \\
$(7.4 \AA)$ & $\left(180^{\circ}\right)$ & $\left(38.9^{\circ}\right)$ & $\left(0^{\circ}\right)$ & $\left(148.1^{\circ}\right)$ & $\left(180^{\circ}\right)$ & $\left(19.7^{\circ}\right)$ \\
13 a.u. & anti & syn & syn & skew & s-trans & s-cis \\
$(6.9 \AA)$ & $\left(180^{\circ}\right)$ & $\left(0^{\circ}\right)$ & $\left(0^{\circ}\right)$ & $\left(167.4^{\circ}\right)$ & $\left(180^{\circ}\right)$ & $\left(0^{\circ}\right)$ \\
12 a.u. & anti & syn & syn & anti & s-trans & s-cis \\
$(6.4 \AA)$ & $\left(180^{\circ}\right)$ & $\left(0^{\circ}\right)$ & $\left(0^{\circ}\right)$ & $\left(180^{\circ}\right)$ & $\left(180^{\circ}\right)$ & $\left(0^{\circ}\right)$ \\
11 a.u. & anti & syn & syn & anti & s-trans & s-cis \\
$(5.8 \AA)$ & $\left(180^{\circ}\right)$ & $\left(0^{\circ}\right)$ & $\left(0^{\circ}\right)$ & $\left(180^{\circ}\right)$ & $\left(180^{\circ}\right)$ & $\left(0^{\circ}\right)$ \\
\hline
\end{tabular}

The trends seen in 1,3-butadiene are similar to the ones shown for n-butane: both in the bulk and within the pores the global energy minimum corresponds to an s-trans conformation, with both methylene groups pointing away from each other at $180^{\circ}$. A gauche conformer, with a $\mathrm{C}-\mathrm{C}-\mathrm{C}-\mathrm{C}$ dihedral angle of $30.3^{\circ}$, is the stable conformer in the bulk. The lower torsion angle (as compared to n-butane) is due to the additional energy 
penalty of breaking the conjugated bond in 1,3-butadiene. As in the previous cases, the non-planar gauche conformation interacts more strongly with the pore walls: its torsion angle is first decreased to $26.7^{\circ}$ in the pore of width 15 a.u., then to $19.7^{\circ}$ in the 14 a.u. pore, and then the $s$-cis planar conformation, at $0^{\circ}$, becomes stable.

n-Butane
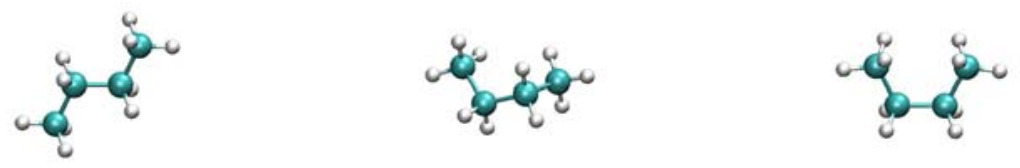

anti

gauche

syn

1-Butene
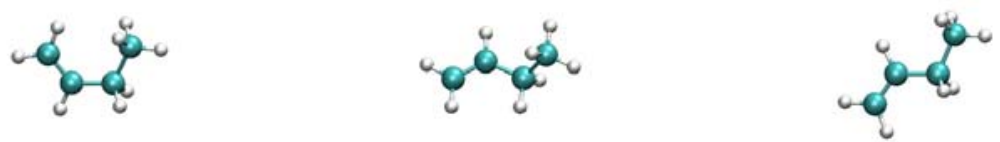

syn

skew

anti

$1,3-$

Butadiene
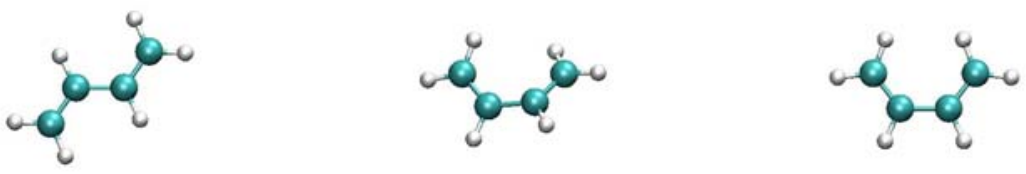

s-trans

gauche

s-cis

Figure 3.1. Snapshots of the stable conformers of n-butane, 1-butene, and 1,3butadiene as obtained from our DFT calculations. The non-planar conformations shown correspond to the bulk molecules.

\subsection{1 n-Butane}

Figure 3.2 shows the torsional profile for an isolated n-butane molecule as obtained from our DFT calculations. We find the torsion angles of the four stationary states to be $180^{\circ}$ 
(anti), $118.6^{\circ}$ (eclipsed), $65.0^{\circ}$ (gauche) and $0^{\circ}$ (syn). We obtain an energy barrier for the anti-gauche transition of $3.1 \mathrm{kcal} / \mathrm{mol}$, and an anti-gauche energy difference of 0.9 $\mathrm{kcal} / \mathrm{mol}$. The anti-syn energy difference is $5.5 \mathrm{kcal} / \mathrm{mol}$. These results are within $1^{\mathrm{o}}$ and $0.5 \mathrm{kcal} / \mathrm{mol}$ of other theoretical calculations ${ }^{25,26,27,28}$. Experimental values for the energy barrier for the anti-gauche transition and for the anti-gauche energy difference are $3.62 \pm 0.06 \mathrm{kcal} / \mathrm{mol}$ and $0.67 \pm 0.10 \mathrm{kcal} / \mathrm{mol}^{29}$, which are in excellent agreement with our results. The estimated experimental value for the anti-syn energy difference is $3.95 \pm 0.03$ $\mathrm{kcal} / \mathrm{mol}$, which is about $1.5 \mathrm{kcal} / \mathrm{mol}$ lower than our calculated difference and other theoretical results. This discrepancy, however, is likely due to the procedure used to interpolate the experimental data, as discussed in ref. 27. We therefore conclude that our calculation method gives a good representation of the torsional profile of n-butane.

The torsional profiles for $n$-butane confined in carbon slit pores with pore widths ranging between 11 a.u. (5.82 $\AA$ ) and 15 a.u. (7.94 $\AA$ ) are shown in figure 3.3. Table 3.2 shows the effect of confinement on the location of the stationary points and their energies.

It is clear from figure 3.3 and table 3.2 that the torsional potential is greatly distorted by confinement, with the main changes being:

- The torsion angle for the gauche conformation decreases with decreasing pore size, and for the pore sizes of 13 a.u. and below the gauche conformer disappears and the syn rotamer becomes stable. This is again a consequence of the steric hindrance imposed by the pore walls, which makes the planar syn conformation more favorable energetically as the pore size decreases.

- A large increase in the barrier for the anti-gauche transition (which becomes anti-syn for pore sizes below 14 a.u.) from $3.1 \mathrm{kcal} / \mathrm{mol}$ in the ideal gas phase to $38.5 \mathrm{kcal} / \mathrm{mol}$ in the smallest pores. The reason for this change is mainly the steric hindrance imposed by the pore walls, which increases the energy of any conformation that is not planar. 
The torsion angle for the eclipsed conformer, however, does not change much with pore size. This suggests that the intramolecular steric hindrance, which determines the location of this maximum, is still dominant in comparison with the constraint imposed by the pore walls (which favors a transition state with a torsion angle closer to $90^{\circ}$ ).

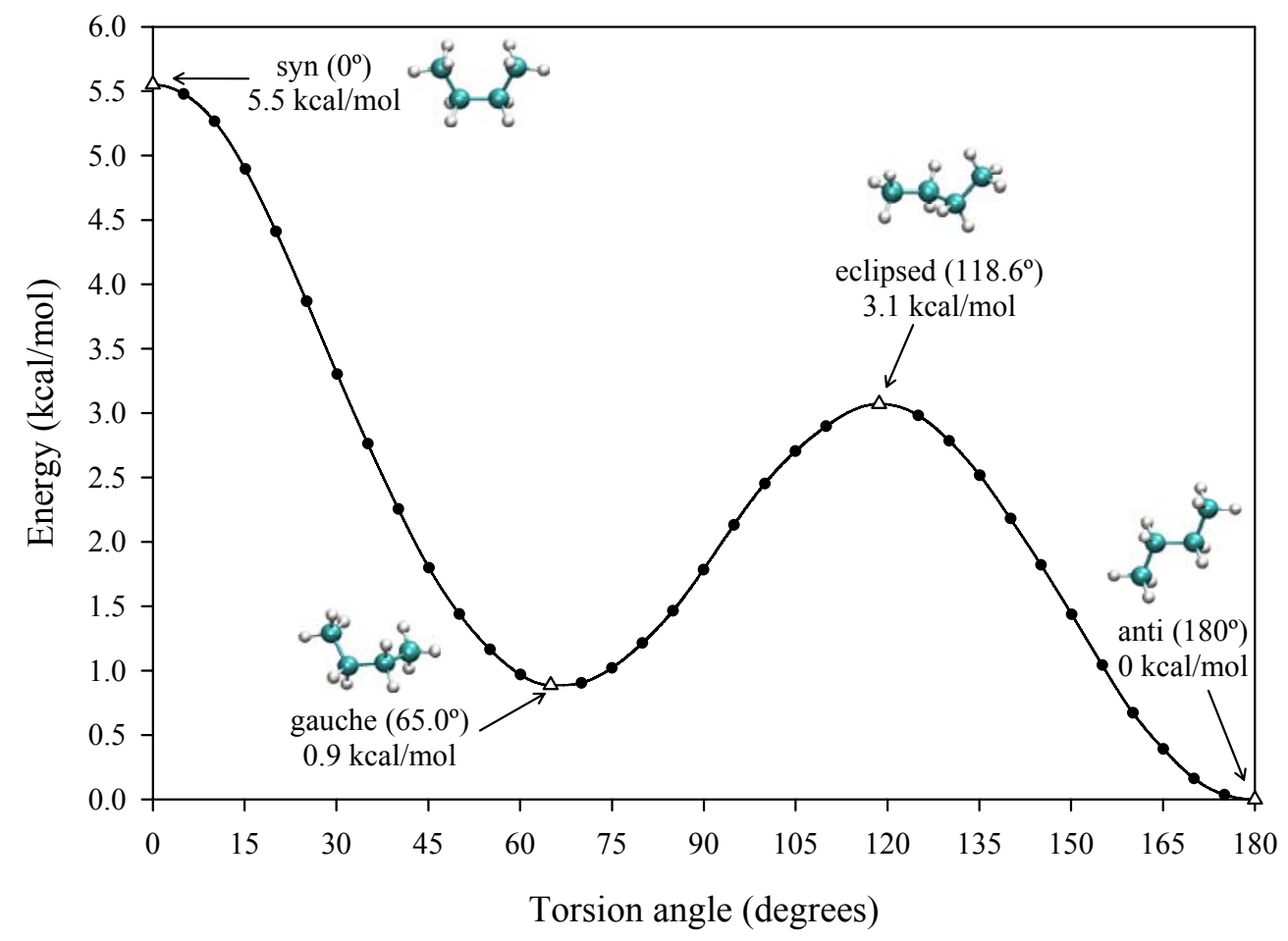

Figure 3.2. Torsional potential of an isolated n-butane molecule as obtained from our DFT-BLYP calculations. The open triangles denote the four stationary points (syn, gauche, eclipsed, and anti), and labels indicate the corresponding values of energy and torsion angle. The black circles denote results of constrained geometry optimizations at various torsion angles. The line is a spline fit to the data. 
- For the smallest pore sizes, the equilibrium distribution of the stable conformers (antisyn) is more favorable toward the anti conformation than it is in the bulk. This is likely due to the intramolecular steric hindrance in the syn conformer, which is higher than that for the gauche conformer as the extreme methyl groups are closer to each other.

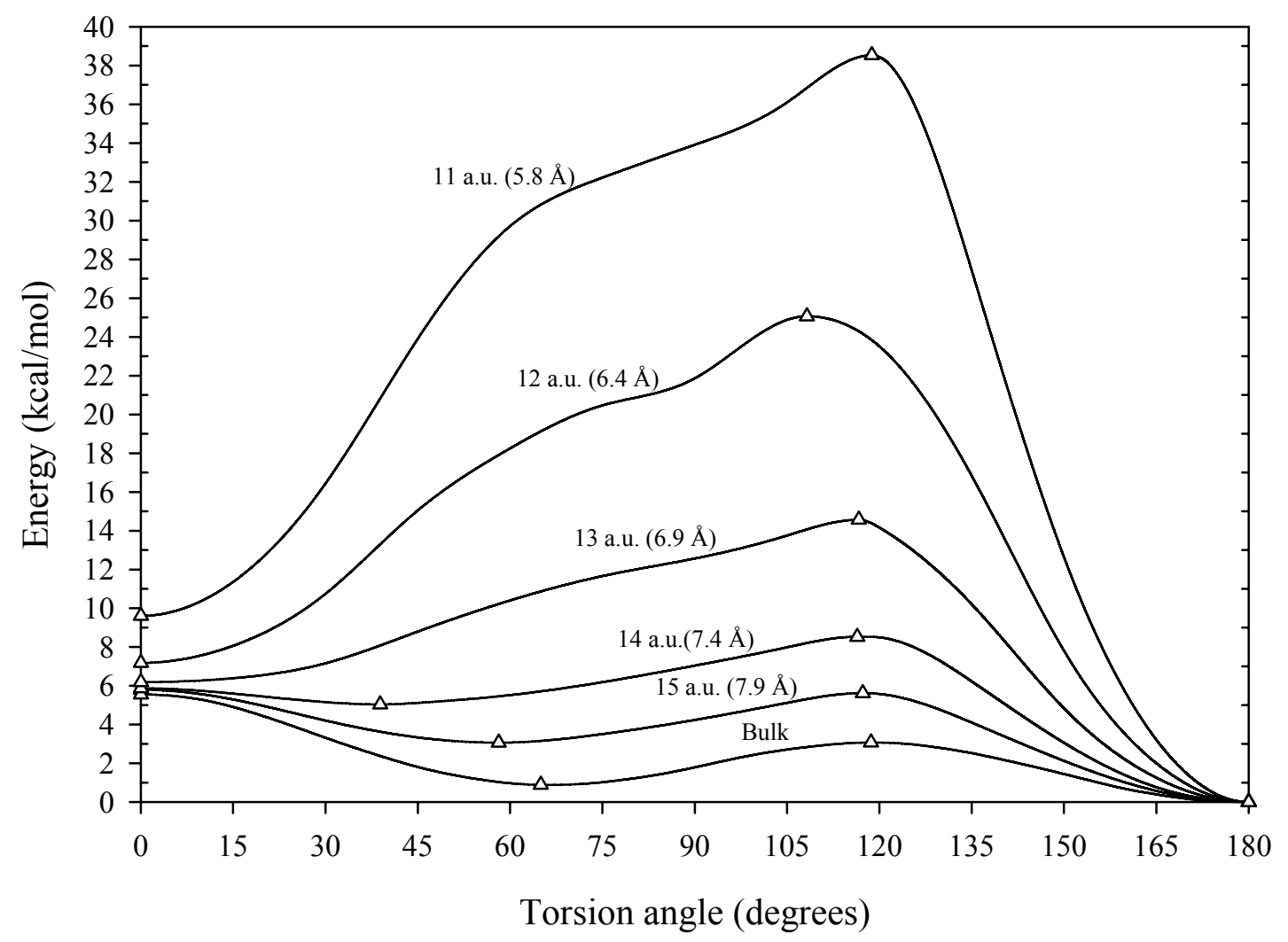

Figure 3.3. Torsional potential of a n-butane molecule confined within carbon slit pores as obtained from our DFT-BLYP calculations. The open triangles denote the stationary points. The lines are spline fits to the data, and labels adjacent to the lines indicate the corresponding pore widths. 
Table 3.2. C-C-C-C dihedral angles $(\omega)$ and energies of the stationary points for the rotational isomerization of $\mathbf{n}$-butane in the gas phase and in carbon slit pores. Energies are in $\mathrm{kcal} / \mathrm{mol}$, and angles in degrees.

\begin{tabular}{cccccc}
\hline Pore width & $\omega_{\text {gauche }}\left({ }^{\circ}\right)$ & $\omega_{\text {eclipsed }}\left({ }^{\circ}\right)$ & $\Delta \mathrm{E}_{\text {anti-gauche }}$ & $\Delta \mathrm{E}_{\text {anti-eclipsed }}$ & $\Delta \mathrm{E}_{\text {anti-syn }}$ \\
\hline Bulk & 65.0 & 118.6 & 0.9 & 3.1 & 5.5 \\
15 a.u. $(7.9 \AA)$ & 58.2 & 117.3 & 3.1 & 5.6 & 5.8 \\
14 a.u. $(7.4 \AA)$ & 38.9 & 116.4 & 5.0 & 8.5 & 5.9 \\
13 a.u. $(6.9 \AA)$ & - & 116.6 & - & 14.6 & 6.2 \\
12 a.u. $(6.4 \AA)$ & - & 108.2 & - & 25.1 & 7.2 \\
11 a.u. $(5.8 \AA)$ & - & 118.8 & - & 38.5 & 9.6 \\
\hline
\end{tabular}

The first two changes illustrate one of the key aspects of the shape-catalytic effect. The reaction for which the transition state is non-planar (i.e. the anti-gauche/anti-syn transition) is severely hindered by confinement in a planar environment, whereas the reaction for which the transition state is planar (i.e. the gauche-gauche automerization, for which the transition state is the syn rotamer), is greatly favored. In fact, the latter is favored so much that it effectively stops being a chemical reaction, because the reaction barrier disappears. We will encounter similar trends in the torsional profiles for 1-butene and 1,3-butadiene.

In order to estimate the impact that these changes due to confinement have on the kinetics for the rotational isomerization of n-butane, we computed the VTST rate constant $k_{V T S T}$ for the anti-gauche/anti-syn transition as a function of temperature both for the ideal gas and pore sizes between 11 a.u. and 14 a.u. The results are shown in figure 3.4 as a set of Arrhenius plots. In this figure, we see that the rate for this transition changes dramatically over a very narrow range of pore sizes, as the rate constants span almost 45 orders of magnitude for temperatures between $200 \mathrm{~K}$ and $800 \mathrm{~K}$. This is also illustrated in table 3.3 , 
which shows the characteristic time (i.e. the inverse of the rate constant) for the transition at $300 \mathrm{~K}$ in the bulk and in the various confined states. Not only is the rate in the smallest pore (11 a.u.) 25 orders of magnitude slower than for the free molecule, also the characteristic time changes over 23 orders of magnitude over a range of pore sizes of $\sim 2 \AA$ only. A simple explanation for such an extreme variation can be given by the following argument. The energy increase due to the overlap of the electron clouds (i.e. the steric hindrance), can be approximated as an exponential ${ }^{30,31}$,

$$
E_{\text {hind }} \approx P \exp (-r / \rho)
$$

where $r$ is the separation between the molecule and the pore walls, and $P$ and $\rho$ are parameters measuring the strength of the interaction and its characteristic length scale. We do not expect this to be very accurate in this case since it assumes, for example, that the molecules in question are spherically symmetric ${ }^{31}$. We will, however, use (3.3.1) as a first approximation. Additionally, we will assume that the steric hindrance imposed on the anti state is negligible compared to the one imposed on the eclipsed state (since anti is planar).

Thus we can write:

$$
\Delta E^{\ddagger} \approx \Delta E_{\infty}^{\ddagger}+P \exp (-r / \rho)
$$

where $\Delta E^{\ddagger}$ is the activation barrier for the reaction, and $\Delta E_{\infty}^{\ddagger}$ is the activation barrier for the isolated molecule. 


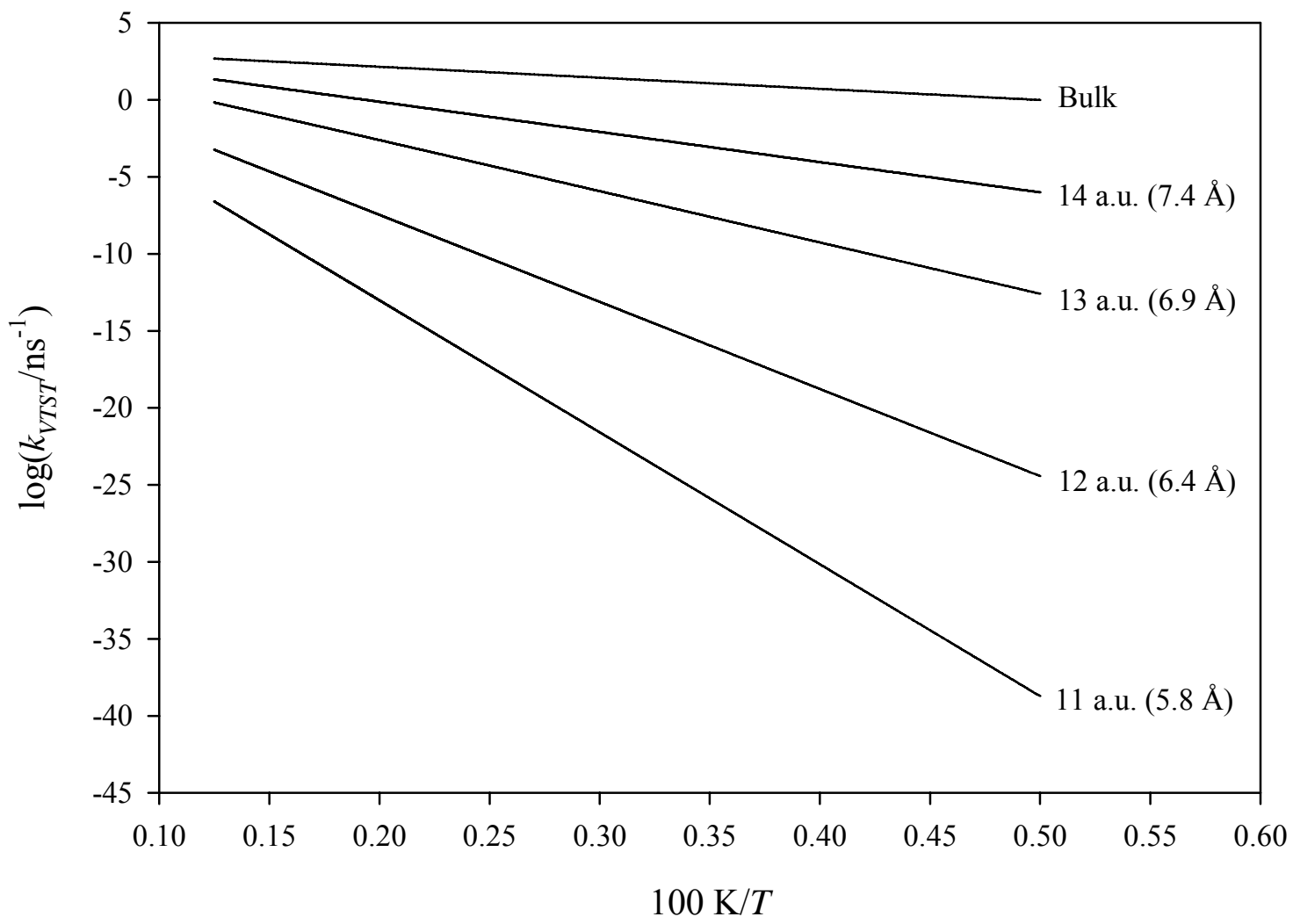

Figure 3.4. Arrhenius plots for the VTST rate constant of the anti-gauche/anti-syn transition in n-butane in the ideal gas phase and within carbon slit pores. The labels to the right of each line indicate the pore size.

If we now assume that transition state theory applies to this reaction ${ }^{32}$, and also that the entropy and volume of activation $\Delta S^{\ddagger}$ and $\Delta V^{\ddagger}$ are approximately constant, we have:

$$
k=A \exp \left[-\frac{\Delta E_{\infty}^{\ddagger}+P \exp (-r / \rho)}{k T}\right]=A^{\prime} \exp \left[-P^{\prime} \exp (-r / \rho)\right]
$$


where $A$ is a frequency factor, and $A^{\prime} \equiv A \exp \left(-\Delta E_{\infty}^{\ddagger} / k T\right)$ and $P^{\prime} \equiv P / k T$ are temperaturedependent parameters. Another way to get this result is to say that the Arrhenius activation energy increases exponentially with decreasing pore size. Thus, as a first approximation, we expect the rate constant to have a doubly exponential dependence on pore size.

Table 3.3. Characteristic time (inverse of the rate constant) for the anti-gauche/antisyn transition of butane at $300 \mathrm{~K}$ in the bulk and within graphene slit pores.

\begin{tabular}{cc}
\hline Pore width & $\tau$ \\
\hline Bulk & $64 \mathrm{ps}$ \\
15 a.u. $(7.9 \AA)$ & $8.0 \mathrm{~ns}$ \\
14 a.u. $(7.4 \AA)$ & $0.5 \mu \mathrm{s}$ \\
13 a.u. $(6.9 \AA)$ & $11 \mathrm{~ms}$ \\
12 a.u. $(6.4 \AA)$ & 12 days \\
11 a.u. $(5.8 \AA)$ & $9 \times 10^{7}$ years \\
\hline
\end{tabular}

From (3.3.3), we can write:

$$
\begin{aligned}
& \ln k=\ln A^{\prime}-P^{\prime} \exp (-r / \rho) \Rightarrow \ln \frac{k_{\infty}}{k}=P^{\prime} \exp (-r / \rho) \Rightarrow \\
& \Rightarrow \ln \left(\ln \frac{k_{\infty}}{k}\right)=\ln P^{\prime}-\frac{r}{\rho}
\end{aligned}
$$

where $k_{\infty}$ is the rate for $r \rightarrow \infty$, i.e. the rate for the isolated molecule. A plot of $\ln \left[\ln \left(k_{\infty} / k\right)\right]$ versus pore size at $300 \mathrm{~K}$ is shown in figure 3.5 . The data fits a straight line 
with a coefficient of determination $R^{2}=0.99$, which is very good considering all the approximations made in obtaining (3.3.4).

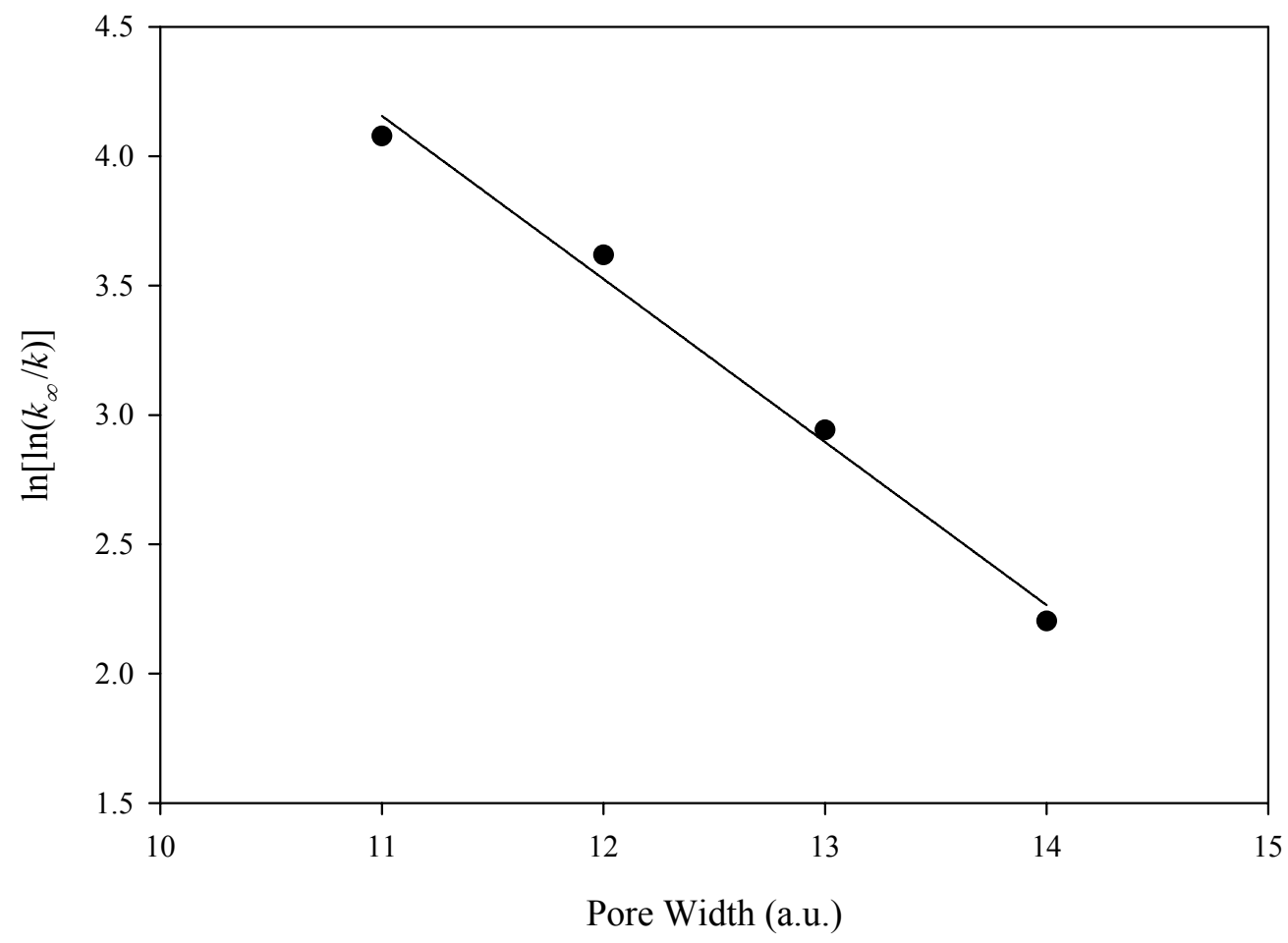

Figure 3.5. The double exponential effect: a plot of $\ln \left[\ln \left(k_{\infty} / k\right)\right]$ vs. pore width at 300 $K$ for $n$-butane using the data from figure 3.4. The black circles correspond to our calculation results, and the line is a linear fit to the data.

From the previous discussion, it is clear that a very small change in the geometry of the confining material can cause a huge change in the dynamics of the confined molecule, as the rate changes roughly as a double exponential of the pore dimensions. This could be used, in principle, to design catalytic materials with greatly improved conversions and selectivities - the geometry should be designed to favor the formation of the transition 
state for the desired reaction, while hindering it for others. It is important to note, however, that there is a trade-off between the enhancement of the desired reaction and the work required to get the molecule inside the pore in the first place. Since the pressure (or, more precisely, the chemical potential) required for the molecule to enter the confined space also increases with the steric hindrance, it would be necessary to adjust the dimensions of the confining material to maximize the reaction enhancement while minimizing the energy required to get the molecule in the pores. Given how sensitive the reaction rate is to the pore dimensions, this would require synthesizing porous materials with very precisely controlled pore sizes.

\subsubsection{1-Butene}

Figure 3.6 shows the torsional profile for an isolated 1-butene molecule as obtained from our DFT calculations. We find the torsion angles of the four stationary states to be $180^{\circ}$ (anti), $121.2^{\circ}$ (skew), 54.4 (gauche) and $0^{\circ}$ (syn). We obtain an energy barrier for the skew-syn transition of $2.4 \mathrm{kcal} / \mathrm{mol}$, and a syn-skew energy difference of $0.6 \mathrm{kcal} / \mathrm{mol}$. The skew-anti energy difference is $2.0 \mathrm{kcal} / \mathrm{mol}$. These results are within $2^{\circ}$ and $0.5 \mathrm{kcal} / \mathrm{mol}$ of other theoretical calculations ${ }^{27,28}$. The skew-anti energy difference calculated from experimental data is $1.75 \pm 0.10 \mathrm{kcal} / \mathrm{mol}^{27,33}$, and the corresponding syn-skew energy difference is $0.15 \pm 0.15 \mathrm{kcal} / \mathrm{mol}$. The agreement between the DFT results and experiment is, in relative terms, slightly worse in this case than it is for n-butane, but the energy differences are also quite small. A more accurate treatment, able to capture these energies precisely, would require a much larger computational effort, which would make the study of the molecule in the pores nearly impossible. Thus, we will accept the DFT results as a reasonable representation of the torsional potential of 1-butene for our purposes. 


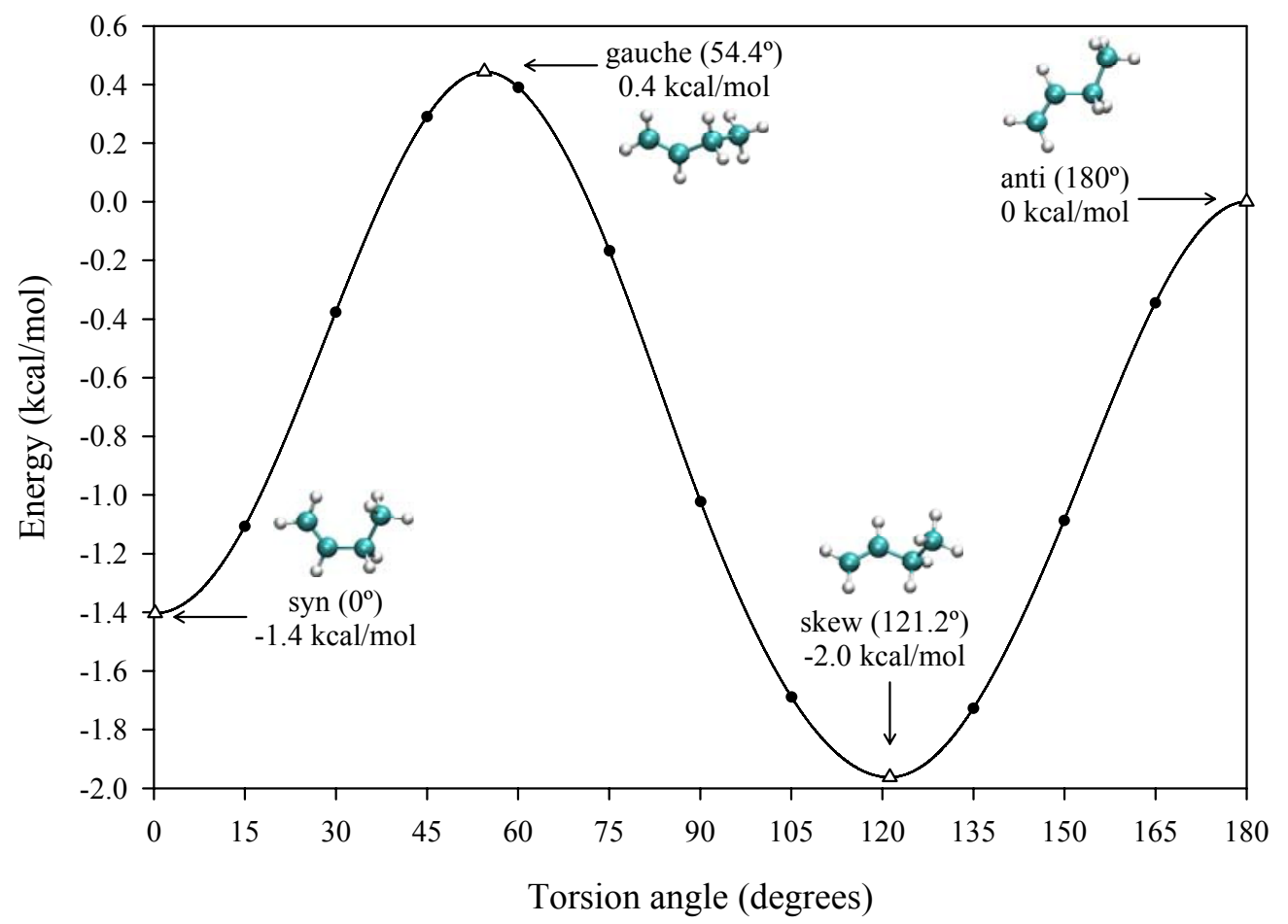

Figure 3.6. Torsional potential of an isolated 1-butene molecule as obtained from our DFT calculations. The open triangles denote the four stationary points (syn, gauche, skew and anti), and labels indicate the corresponding values of energy and torsion angle. The black circles denote results of constrained geometry optimizations at various torsion angles. The line is a spline fit to the data.

The torsional profiles for 1-butene confined in carbon slit pores with pore widths ranging between 11 a.u. (5.82 $\AA$ ) and 15 a.u. (7.94 $\AA$ ) are shown in figure 3.7. Table 3.4 shows the effect of confinement on the location of the stationary points and their energies. 
As was the case for n-butane, figure 3.7 and table 3.4 show a large effect on the torsional profile as a result of confinement. The main changes are:

- The torsion angle for the skew conformation increases with decreasing pore size, and for the pore sizes of 12 a.u. and below the skew conformer disappears and the anti rotamer becomes stable (for the 13 a.u. pores the torsional profile becomes quite flat near the anti state, and it is hard to tell whether there is still a stable skew conformer). This is again a consequence of the steric hindrance imposed by the pore walls, which makes the planar anti conformation more favorable energetically as the pore size decreases.

- A large increase in the barrier for the skew-syn transition (which becomes the anti-syn for pore sizes below 13 a.u.) from $2.4 \mathrm{kcal} / \mathrm{mol}$ in the ideal gas phase to $27.8 \mathrm{kcal} / \mathrm{mol}$ in the smallest pores. This is analogous to what happens to the anti-syn transition in nbutane: since the gauche transition state is not planar, the steric hindrance imposed by the pore walls increases its energy. The torsion angle for the gauche transition state, unlike that for the eclipsed state in butane, does change quite a lot due to confinement: from $54.4^{\circ}$ in the bulk to $102.5^{\circ}$ in the smallest pore. This indicates that in the case of 1-butene, having one methylene and one methyl end groups, the intramolecular steric hindrance is not as important as it is in the case of n-butane, which has two (more bulky) methyl groups. Thus the constraint imposed by the pore walls dominates and causes the transition structure to be closer to $90^{\circ}$.

- For the smaller pore sizes, the equilibrium distribution of the stable conformers (skewsyn) becomes more favorable toward the syn conformation than it is in the bulk. This is probably because of the intramolecular steric hindrance in the anti conformer, which is a stable state at the smaller pore sizes. 


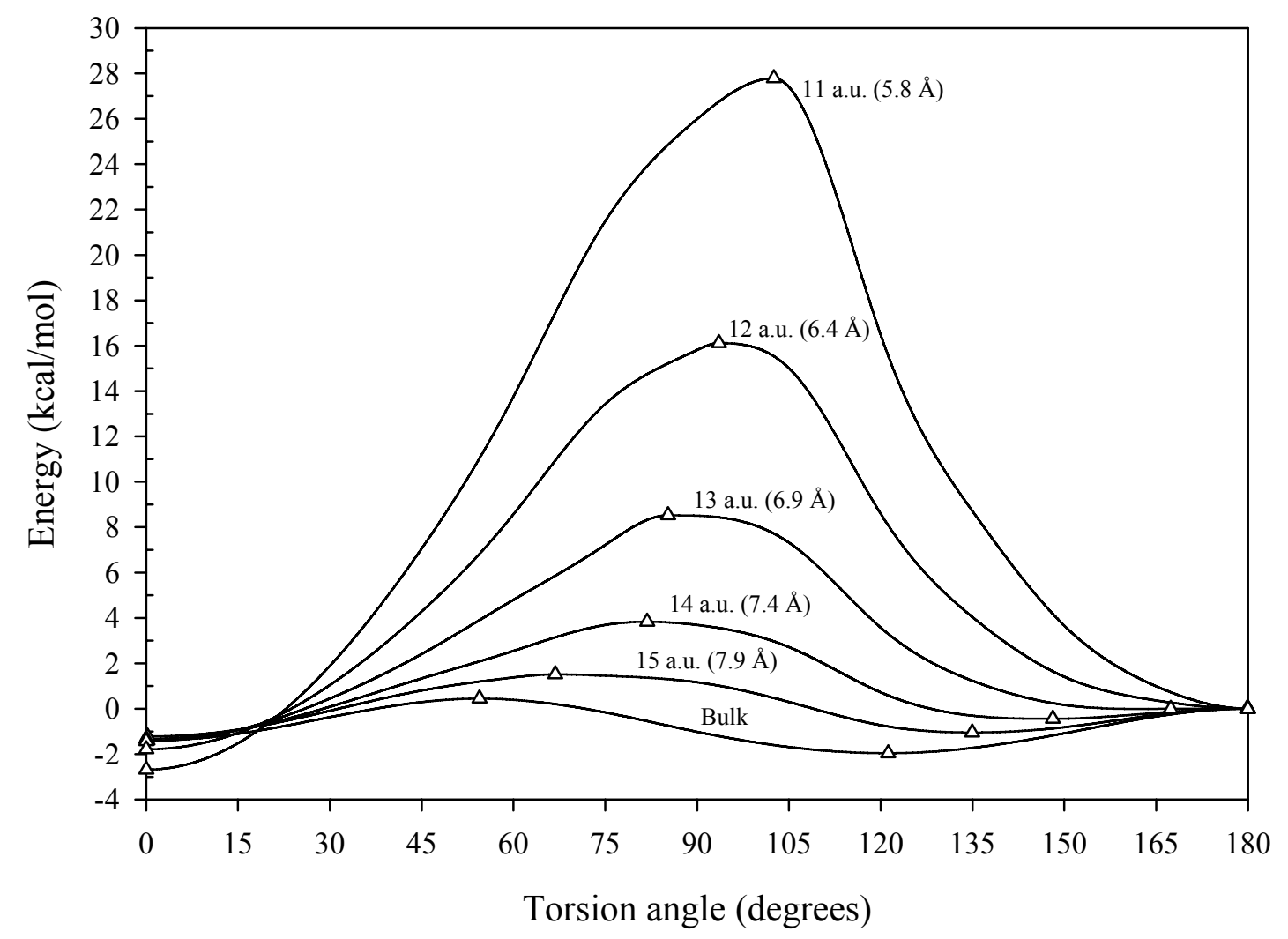

Figure 3.7. Torsional potential of a 1-butene molecule confined within carbon slit pores as obtained from our DFT-BLYP calculations. The open triangles denote the stationary points. The lines are spline fits to the data, and labels adjacent to the lines indicate the corresponding pore widths.

These changes show, as in the case of n-butane, that the geometrical restrictions imposed on the confined molecule by the pore walls hinder the reaction for which the transition state is not planar (skew to syn), and enhance the one for which the transition state is planar (skew-skew automerization), eventually causing the latter to not be a reaction and the former transition state (anti) to be stable. It is interesting to note that, despite the fact that the torsional potential for the isolated 1-butene molecule is very different qualitatively 
from the one for n-butane, both molecules have quite similar torsional potentials in the pores. This shows that, for small enough pore sizes it is really the structure of the confining material that determines the potential energy surface rather than the structure of the reacting molecule. A similar trend will be seen for 1,3-butadiene.

Table 3.4. C-C-C-C dihedral angles $(\omega)$ and energies of the stationary points for the rotational isomerization of 1-butene in the gas phase and in carbon slit pores. Energies are in $\mathrm{kcal} / \mathrm{mol}$, and angles in degrees.

\begin{tabular}{|c|c|c|c|c|c|}
\hline Pore width & $\omega_{\text {gauche }}\left({ }^{\circ}\right)$ & $\omega_{\text {skew }}\left({ }^{o}\right)$ & $\Delta \mathrm{E}_{\text {skew-syn }}$ & $\Delta \mathrm{E}_{\text {skew-gauche }}$ & $\Delta \mathrm{E}_{\text {skew-ant }}$ \\
\hline Bulk & 54.4 & 121.2 & 0.6 & 2.4 & 2.0 \\
\hline $\begin{array}{l}15 \text { a.u. } \\
(7.9 \AA)\end{array}$ & 66.9 & 135.0 & 0.2 & 2.6 & 1.0 \\
\hline $\begin{array}{l}14 \text { a.u. } \\
\text { (7.4 } \AA)\end{array}$ & 81.8 & 148.1 & -0.9 & 4.3 & 0.4 \\
\hline $\begin{array}{l}13 \text { a.u. } \\
(6.9 \AA)\end{array}$ & 85.3 & 167.4 & -1.4 & 8.5 & $<0.1$ \\
\hline $\begin{array}{l}12 \text { a.u. } \\
(6.4 \AA)\end{array}$ & 93.6 & - & -1.8 (anti-syn) & 16.1 (anti-gauche) & - \\
\hline $\begin{array}{l}11 \text { a.u. } \\
(5.8 \AA)\end{array}$ & 102.5 & - & -2.7 (anti-syn) & 27.8 (anti-gauche) & - \\
\hline
\end{tabular}

As we did for n-butane, we computed the VTST rate constant for the pore widths ranging from 11 a.u. to 14 a.u. The results are shown in figure 3.8 as a set of Arrhenius plots. The results are similar to those obtained for n-butane, although the more drastic changes happen at smaller pore sizes, reflecting the fact that 1-butene is a smaller molecule. Nevertheless, the variation of the rate with pore size is still quite extreme - for example, the characteristic time for the asymmetric isomerization $(s y n \rightarrow$ skew/syn $\rightarrow$ anti) changes 
from an estimated $2 \mathrm{ps}$ in the bulk to $0.27 \mathrm{~ns}$ in the 14 a.u.-wide pore and to 430 years in the 11 a.u.-wide pore. The variation is still over more than 20 orders of magnitude.

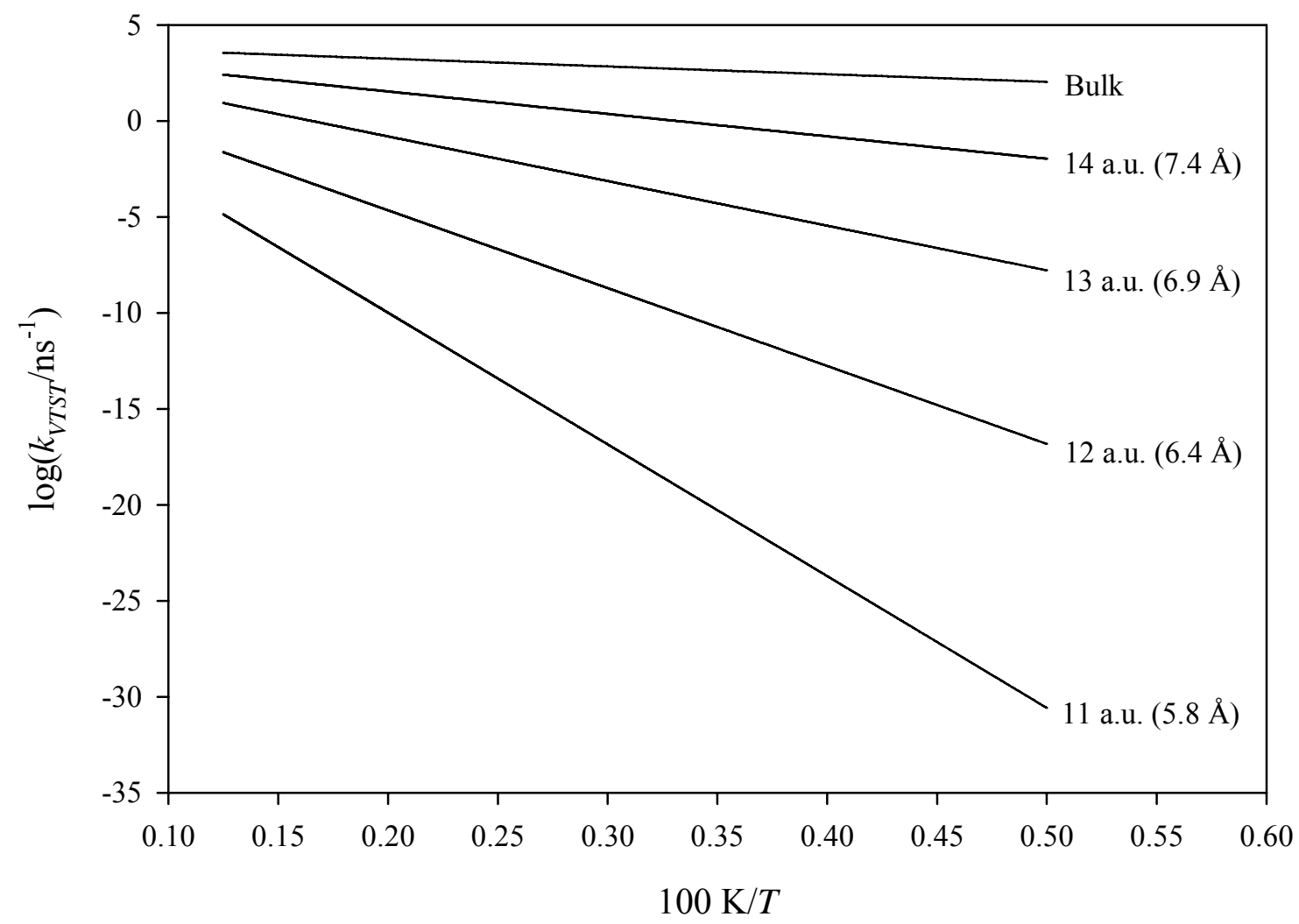

Figure 3.8. Effect of confinement on the isomerization rate of 1-butene as obtained from our VTST calculations. The line labeled "Bulk" corresponds to the isolated molecule (ideal gas). The other lines correspond to isolated molecules confined in carbon slit pores. The labels next to the lines indicate the corresponding pore widths.

In order to illustrate the double exponential effect, figure 3.9 shows a plot of $\ln \left[\ln \left(k_{\infty} / k\right)\right]$ versus pore size for 1 -butene at $300 \mathrm{~K}$. As in the case of n-butane, the data 
fits a straight line with a coefficient of determination $R^{2}=0.99$, which again suggests that equation (3.3.4) gives a good representation of the confinement effect in this range of pore sizes.

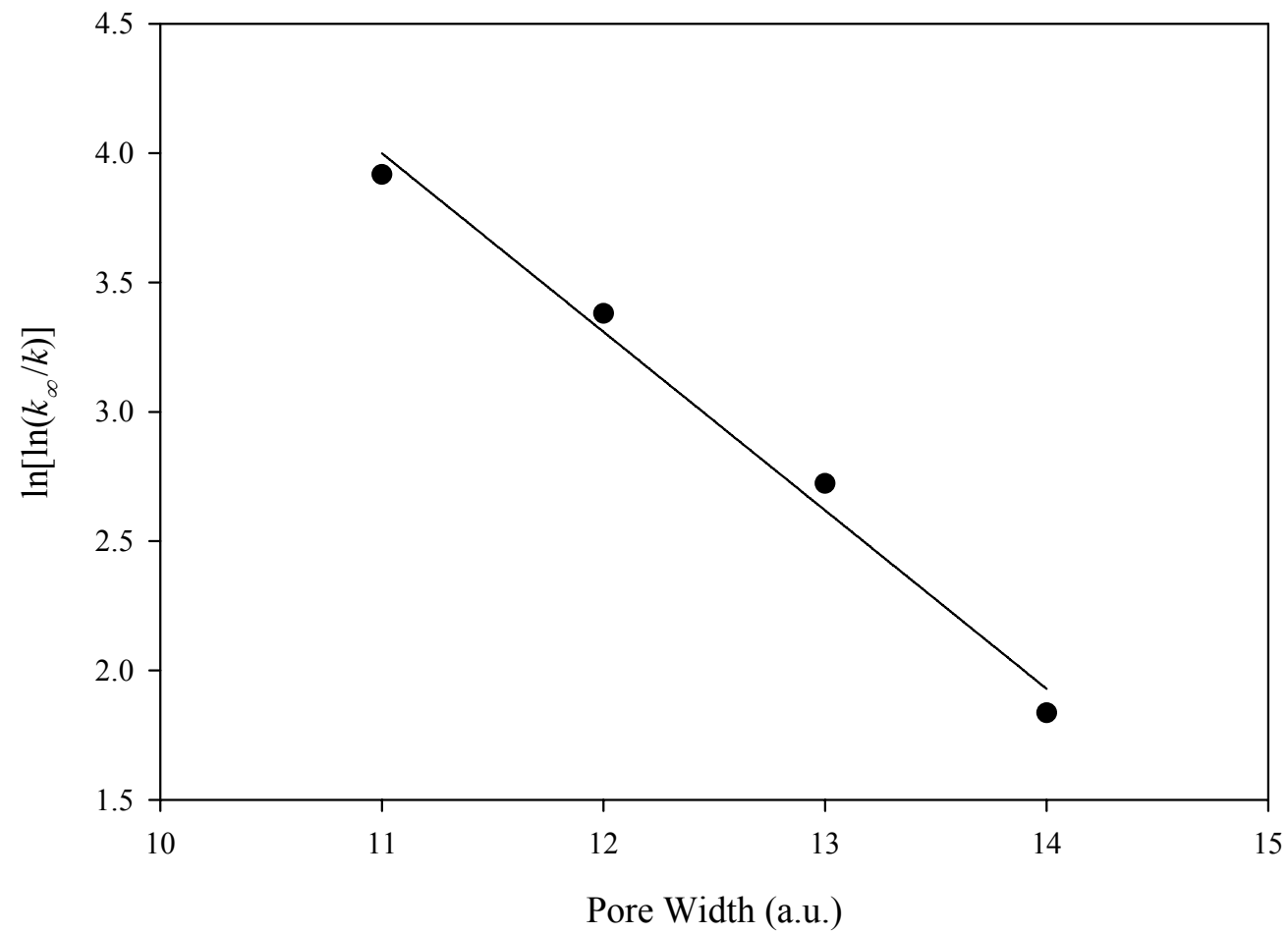

Figure 3.9. The double exponential effect: a plot of $\ln \left[\ln \left(k_{\infty} / k\right)\right]$ vs. pore width at 300 $\mathrm{K}$ for 1-butene using the data from figure 3.8. The black circles correspond to our calculation results, and the line is a linear fit to the data. 


\subsubsection{1,3-Butadiene}

The torsional profile for an isolated 1,3-butadiene molecule, as obtained from our DFT calculations, is shown in figure 3.10. This profile is a refined version of the one in refs. 1, 2.

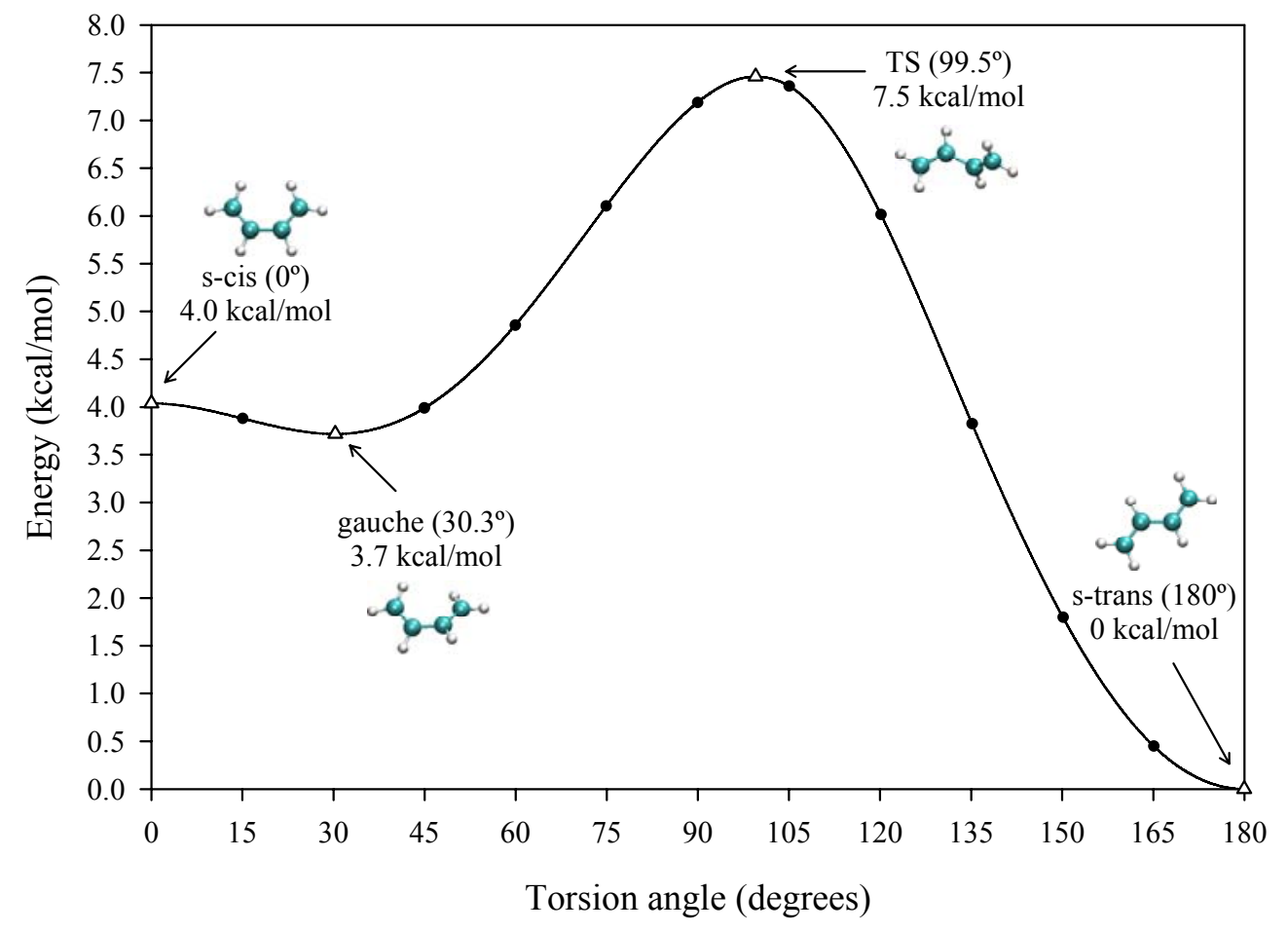

Figure 3.10. Torsional potential of an isolated 1,3-butadiene molecule as obtained from our DFT-BLYP calculations. The open triangles denote the four stationary points (s-cis, gauche, TS and s-trans), and labels indicate the corresponding values of energy and torsion angle. The black circles denote results of constrained geometry optimizations at various torsion angles. The line is a spline fit to the data. 
We find the torsion angles of the four stationary points to be $180^{\circ}$ (s-trans), $99.5^{\circ}$ (TS, for "transition state"), $30.3^{\circ}$ (gauche) and $0^{\circ}$ (s-cis). We obtain an energy barrier for the strans-gauche transition of $7.5 \mathrm{kcal} / \mathrm{mol}$, and an energy difference of $3.7 \mathrm{kcal} / \mathrm{mol}$ between the gauche and the s-trans conformers. The s-trans-s-cis energy difference is $4.0 \mathrm{kcal} / \mathrm{mol}$, and the gauche-s-cis energy difference is $0.3 \mathrm{kcal} / \mathrm{mol}$. The energy difference between the s-trans and the s-cis conformers, as well as the difference between the s-trans and gauche conformers and the location of the TS state, are in good agreement with both experimental $^{34}$ and theoretical ${ }^{35,36}$ results.

The height of the s-trans-gauche barrier shown in figure 3.10 is, however, overestimated by about $1.6 \mathrm{kcal} / \mathrm{mol}$ (as compared to experiment), and the torsion angle for the gauche conformer is predicted to be about $3-5^{\circ}$ lower than the best theoretical estimates (using coupled-cluster theory ${ }^{36}$ ). The overestimation by $1-2 \mathrm{kcal} / \mathrm{mol}$ of the barrier height for conjugated bond breaking is a known drawback of the Becke exchange ${ }^{36-38}$, but this error is much smaller than the effect of confinement on the torsional profile. The difference in the torsion angle for the gauche conformer among different theoretical estimates is related to the fact that the torsional potential becomes nearly flat in that region, which makes it very difficult to locate the minimum precisely. Since a more accurate treatment would make the problem intractable when considering the rotation within the pores, we will accept our DFT results as a reasonable representation of the torsional potential of 1,3-butadiene for the purpose of studying the effect of confinement.

The torsional profiles for 1,3-butadiene confined in carbon slit pores with pore widths ranging between 11 a.u. (5.82 $\AA$ ) and 15 a.u. (7.94 $\AA$ ) are shown in figure 3.11. Table 3.5 shows the effect of confinement on the location of the stationary points and their energies. As was the case for n-butane and 1-butene, confinement greatly changes the torsional profile. The changes are analogous to what we observed for the previous cases: 
- The torsion angle for the gauche conformer decreases with pore size, and for pore sizes of 13 a.u. and lower the gauche conformer disappears, and the s-cis conformer becomes stable (previous simulations ${ }^{1,2}$ ) had predicted a very shallow gauche minimum at $15.2^{\circ}$ in the 13 a.u. pore, but this minimum disappeared upon refining the calculations). This is analogous to what happened to the gauche state in n-butane and the skew state in 1-butene: the steric hindrance imposed by the pore walls makes the planar s-cis conformation more stable than the non-planar gauche conformation. In the particular case of 1,3-butadiene, the transformation of the gauche structure in the gas phase to a s-cis structure when confined in an Argon matrix has been studied in the past due to a controversy regarding the correct structure of the high-energy stable rotamer ${ }^{39,40}$. Our results indicate a similar trend in the graphene pores.

- The barrier for the s-trans-gauche transition (which becomes the s-trans-s-cis for pore sizes below 14 a.u.) increases from $7.5 \mathrm{kcal} / \mathrm{mol}$ in the ideal gas state to $29.5 \mathrm{kcal} / \mathrm{mol}$ in the smallest pore. This is analogous to the changes in the anti-gauche transition in butane and the skew-syn transition in 1-butene: since the TS state is not planar, its energy is increased rapidly due to the steric hindrance imposed by the pore walls. The torsion angle for the transition state does not change much due to confinement, indicating that the predominant influence in its location is the intramolecular steric hindrance.

- The equilibrium distribution of the s-trans and gauche (s-trans and s-cis for pore sizes smaller than 14 a.u.) remains approximately the same regardless of pore size, unlike the case for n-butane and 1-butene. The reason for this difference is the much smaller intramolecular steric hindrance in the s-cis conformation of 1,3-butadiene, as compared to the syn conformer of n-butane and the anti conformer of 1-butene. Since this factor is not as important in this case, confinement does not have a strong influence in the energy difference between s-trans and s-cis, as they are both planar structures. 


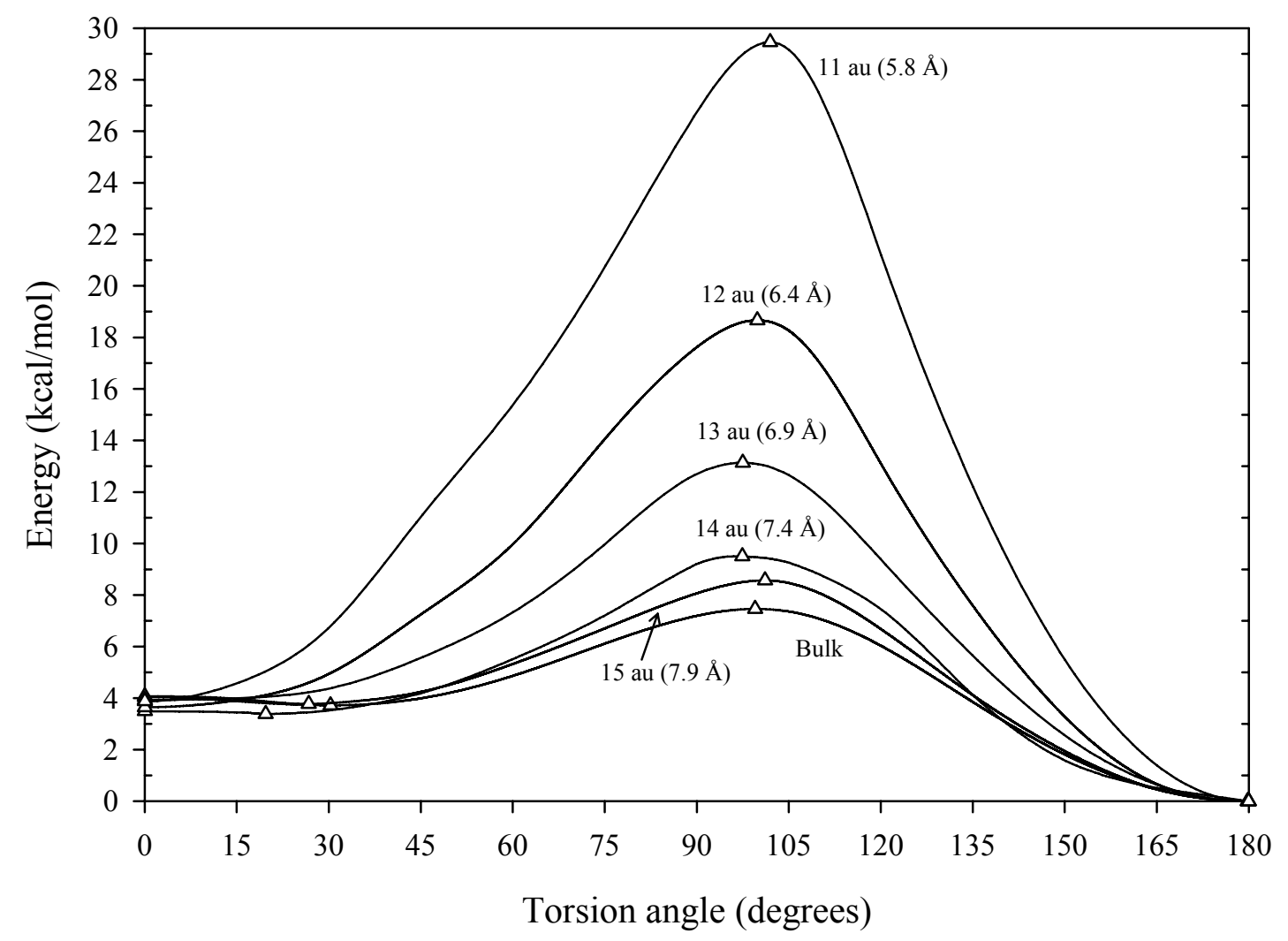

Figure 3.11. Torsional potential of a 1,3-butadiene molecule confined within carbon slit pores as obtained from our DFT-BLYP calculations. The open triangles denote the stationary points. The lines are spline fits to the data, and labels adjacent to the lines indicate the corresponding pore widths.

As we observed for n-butane and 1-butene, the effect of confinement is again to hinder the transition for which the transition state is not planar (s-trans to gauche), and to enhance the transition for which it is planar (gauche automerization). As in the previous cases, the gauche automerization is not even a reaction, as the transition state (s-cis) becomes stable. We also observe again that the potential energy surface in the smaller pores is determined by the geometry of the pore space rather than the confined molecule: all of the torsional 
profiles studied become like a cis-trans profile in the smallest pores, despite the different bonding structures and bulk torsional potentials of the molecules involved.

Table 3.5. C-C-C-C dihedral angles $(\omega)$ and energies of the stationary points for the rotational isomerization of 1,3 -butadiene in the gas phase and in carbon slit pores.

Energies are in $\mathrm{kcal} / \mathrm{mol}$, and angles in degrees.

\begin{tabular}{|c|c|c|c|c|c|}
\hline Pore width & $\omega_{\text {gauche }}\left({ }^{\circ}\right)$ & $\omega_{\mathrm{TS}}\left({ }^{\mathrm{o}}\right)$ & $\Delta \mathrm{E}_{\text {s-trans-gauche }}$ & $\Delta \mathrm{E}_{s \text {-trans-TS }}$ & $\Delta \mathrm{E}_{\text {s-trans-s-cis }}$ \\
\hline Bulk & 30.3 & 99.5 & 3.7 & 7.5 & 4.0 \\
\hline $\begin{array}{l}15 \text { a.u. } \\
(7.9 \AA)\end{array}$ & 26.7 & 101.2 & 3.8 & 8.6 & 4.1 \\
\hline $\begin{array}{l}14 \text { a.u. } \\
(7.4 \AA)\end{array}$ & 19.7 & 97.4 & 3.4 & 9.5 & 3.5 \\
\hline $\begin{array}{l}13 \text { a.u. } \\
(6.9 \AA)\end{array}$ & - & 97.5 & - & 13.1 & 3.9 \\
\hline $\begin{array}{l}12 \text { a.u. } \\
(6.4 \AA)\end{array}$ & - & 99.9 & - & 18.7 & 3.6 \\
\hline $\begin{array}{l}11 \text { a.u. } \\
(5.8 \AA)\end{array}$ & - & 102.0 & - & 29.5 & 3.9 \\
\hline
\end{tabular}

As we did for n-butane and 1-butene, we computed the VTST rate constant for the pore widths ranging from 11 a.u. to 14 a.u. The results are shown in figure 3.12 as a set of Arrhenius plots. The results follow the same trends described for n-butane and 1-butene. Since this is the smallest molecule considered, the larger part of the variation in the rates happens at smaller pore widths than for n-butane and 1-butene, again reflecting that the important parameter is the molecular size compared to the characteristic size of the confining material. We still see, however, extreme variations of the rate with pore width, with the characteristic time for the s-trans $\rightarrow$ gauche/s-trans $\rightarrow$ s-cis transition at $300 \mathrm{~K}$ 
varying from $17 \mathrm{~ns}$ in the bulk to over 12 years in the smallest pore, a change of 16 orders of magnitude.

In order to illustrate the double exponential effect for 1,3-butadiene, figure 3.13 shows a plot of $\ln \left[\ln \left(k_{\infty} / k\right)\right]$ versus pore size at $300 \mathrm{~K}$. In this case the data fits a straight line with a coefficient of determination $R^{2}=0.97$, slightly worse than what was obtained for the other two hydrocarbons. The larger deviation from linear behavior comes from the pore at the largest pore size (14 a.u.), where it is likely that the steric hindrance from the pore walls is not the only important contribution, as it was assumed in the derivation of equation (3.3.4). Nevertheless, considering the simplicity of the theory behind (3.3.4), the agreement seen in figure 3.13 is still quite good. We expect that such a dependence of kinetics on pore size will be found for any systems where the overlap interaction dominates over other factors, and for which the Arrhenius equation is an adequate representation of the variation of the rate with the activation barrier. This includes most systems in which the characteristic dimensions of the confining material are comparable to the molecular size. 


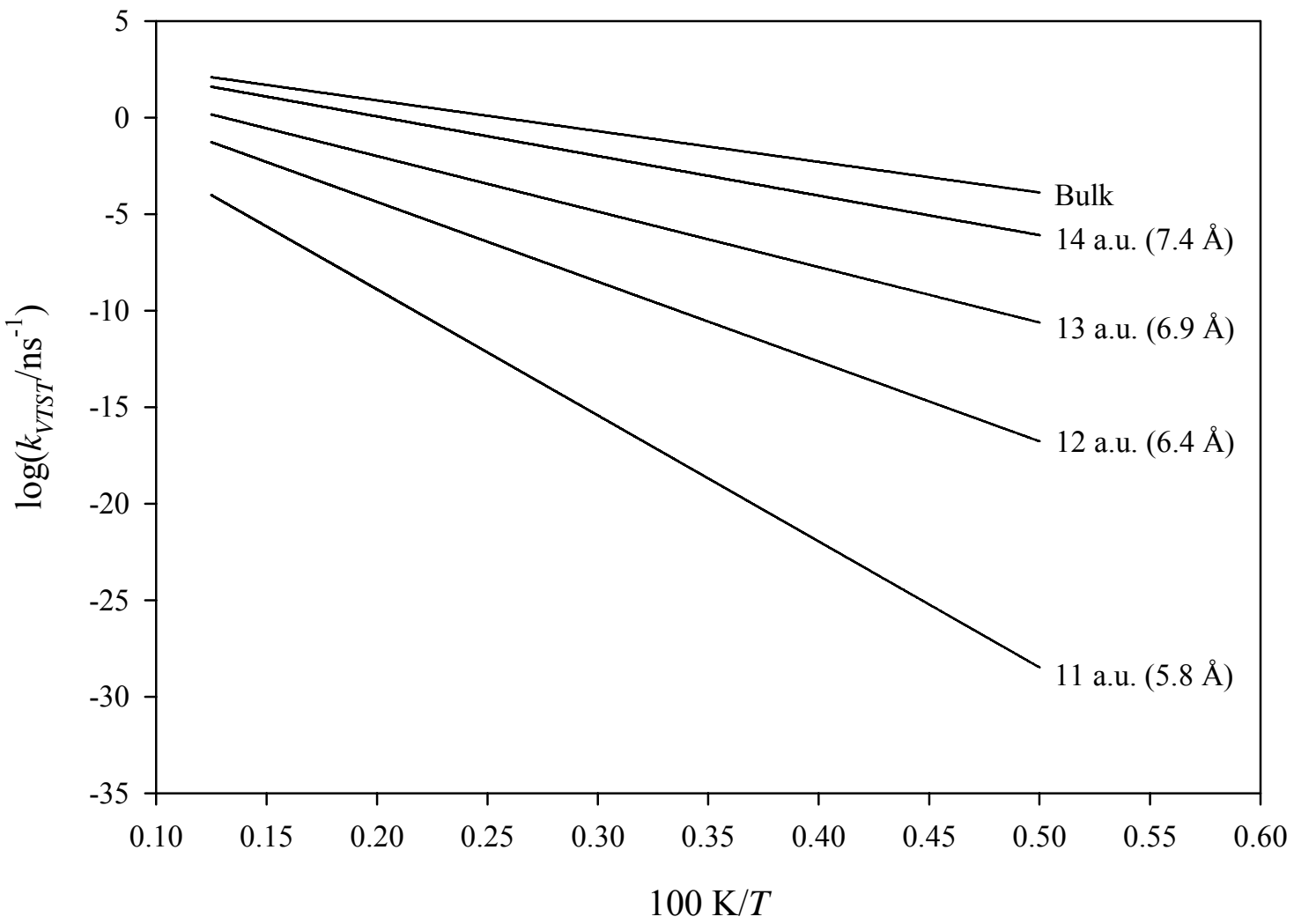

Figure 3.12. Effect of confinement on the isomerization rate of 1,3-butadiene as obtained from our VTST calculations. The line labeled "Bulk" corresponds to the isolated molecule (ideal gas). The other lines correspond to isolated molecules confined in carbon slit pores. The labels next to the lines indicate the corresponding pore widths. 


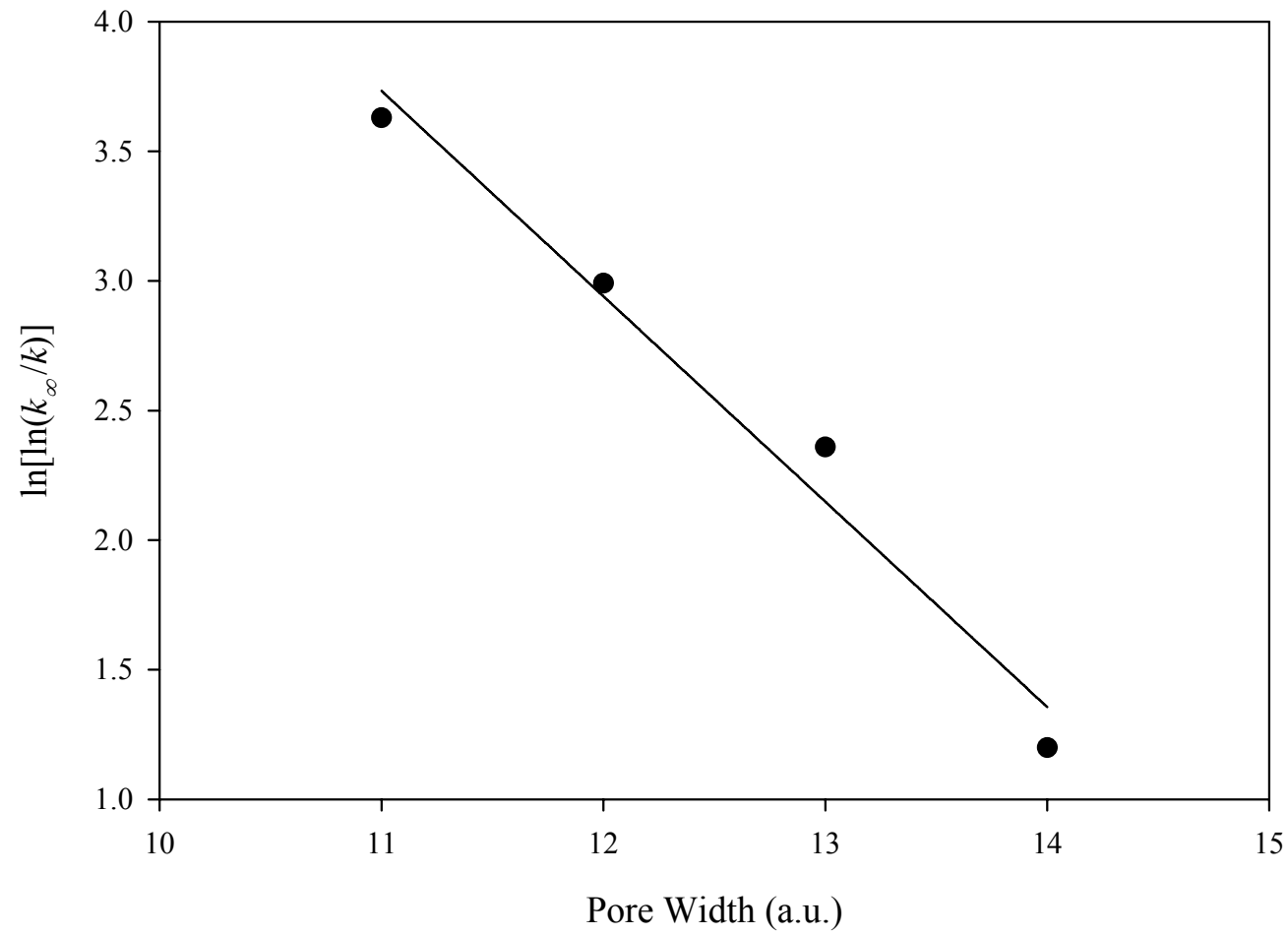

Figure 3.13. The double exponential effect: a plot of $\ln \left[\ln \left(k_{\infty} / k\right)\right]$ vs. pore width at 300 $K$ for 1,3-butadiene using the data from figure 3.12. The black circles correspond to our calculation results, and the line is a linear fit to the data

\subsection{Concluding Remarks}

Our results show that the torsional potentials of n-butane, 1-butene and 1,3-butadiene are greatly altered by confinement when the pore width of the confining material becomes comparable to the molecular dimensions. Despite the different bonding structures and gasphase torsional potentials of the three molecules, all are affected in an analogous way by confinement, thus showing that the potential energy surface is determined mainly by the 
geometry of the porous space. Therefore, we expect that other molecules would be affected by confinement in a similar way.

Our calculations also indicate that the isomerization dynamics of n-butane, 1-butene and 1,3-butadiene are dramatically affected by confinement in the molecular sieving limit, i.e. when the pore size of the confining material becomes comparable to the molecular size. In spite of the differences in the bonding structure of the three molecules, they all show the same behavior within the smallest pores - their torsional potentials shift to an anti-syn (or cis-trans) form, and the isomerization rates vary by many orders of magnitude in a very narrow range of pore sizes. Our results are consistent with a double exponential dependence of the reaction rate with pore width, independently of the details of the confined molecule. We expect this type of behavior to be seen in most systems where the overlap interaction with the pore walls is the dominant interaction, i.e. for sufficiently small pore sizes. If this is indeed the case, this shape-catalytic effect could be used to design much improved catalytic supports using flexible materials such as graphitic carbon. It is likely, however, that there is a trade-off between the work required to get the molecule into the pore and the catalytic enhancement. Our current studies of the influence of the thermodynamic state of the adsorbed phase on the dynamics of the confined molecule will shed light on this aspect of the problem. 


\section{References and Notes}

${ }^{1}$ E.E. Santiso, A.M. George, C.H. Turner, M.K. Kostov, K.E. Gubbins, M. Buongiorno Nardelli, M.

Sliwinska-Bartkowiak, Appl. Surf. Sci. 252, 766 (2005).

${ }^{2}$ E.E. Santiso, A.M. George, M. Sliwinska-Bartkowiak, M. Buongiorno Nardelli, K.E. Gubbins, Adsorption 11, 349 (2005).

${ }^{3}$ E.E. Santiso, M. Buongiorno Nardelli, K.E. Gubbins, Adsorption, submitted (2007).

${ }^{4}$ E.E. Santiso, M. Buongiorno Nardelli, K.E. Gubbins, J. Chem. Phys., submitted (2007).

${ }^{5}$ E.E. Santiso, M.K. Kostov, A.M. George, M. Buongiorno Nardelli, K.E. Gubbins, Appl. Surf. Sci. 253, 5570 (2007).

${ }^{6}$ E.E. Santiso, L. Huang, K.E. Gubbins, M.K. Kostov, A.M. George and Marco Buongiorno Nardelli, “Ab initio simulations of chemical reactions in nanostructured carbon materials", in Quantum Chemical Calculations of Surfaces and Interfaces of Materials, American Scientific Publishers, Valencia, California (2007).

${ }^{7}$ R.G. Parr, W. Yang, Density-Functional Theory of Atoms and Molecules, Oxford University Press, Oxford (1989).

${ }^{8}$ E.E. Santiso, K.E. Gubbins, Mol. Simul. 30, 699 (2004).

${ }^{9}$ A.D. Becke, Phys. Rev. A 38, 3098 (1988).

${ }^{10}$ C. Lee, W. Yang, R.G. Parr, Phys. Rev. B 37, 785 (1988).

${ }^{11}$ D. Vanderbilt, Phys. Rev. B 41, 7892 (1990).

${ }^{12}$ CPMD, Copyright IBM Corp 1990-2006, Copyright MPI für Festkörperforschung Stuttgart 1997-2001. http://www.cpmd.org.

${ }^{13}$ M. Elstner, P. Hobza, T. Frauenheim, S. Suhai, E. Kaxiras, J. Chem. Phys. 114, 5149 (2001).

${ }^{14}$ W.T.M. Mooij, F.B. van Duijneveldt, J.G.C.M. van Duijneveldt-van de Rijdt, B.P. van Eijck, J. Phys. Chem. A 103, 9872 (1999). 
${ }^{15}$ R.W. Williams, D. Malhotra, Chem. Phys. 327, 54 (2006).

${ }^{16}$ A. Banerjee, N. Adams, J. Simons, R. Shepart, J. Phys. Chem. 89, 52 (1985).

${ }^{17}$ S.R. Billeter, A. Curioni, W. Andreoni, Comp. Mater. Sci. 27, 437 (2003).

${ }^{18}$ See, for example, J. Simons, An Introduction to Theoretical Chemistry, Cambridge University Press, Cambridge (2003).

${ }^{19}$ ParFuMS (Partition Functions of Molecules and Solids), E.E. Santiso, 2007. The full source code for the program as well as extensive documentation and examples, can be found at the beta release web site, http://gubbins.ncsu.edu/users/erik/parfums/.

${ }^{20}$ T.M. Reed, K.E. Gubbins, Applied Statistical Mechanics, Butterworth-Heinemann, Boston (1973).

${ }^{21}$ C.G. Gray, K.E. Gubbins, Molecular Theory of Fluids Volume 1: Fundamentals, Clarendon Press, Oxford (1984).

${ }^{22}$ M. Page, J.W. McIver, Jr., J. Chem. Phys. 88, 922 (1988).

${ }^{23}$ J. González, X. Giménez, J.M. Bofill, Theor. Chem. Acc. 112, 75 (2004).

${ }^{24}$ E.E. Santiso, K.E. Gubbins, in preparation (2007).

${ }^{25}$ K. Raghavachari, J. Chem. Phys. 81, 1383 (1984).

${ }^{26}$ K.B. Wiberg, M.A. Murcko, J. Am. Chem. Soc. 110, 8029 (1988).

${ }^{27}$ M.A. Murcko, H. Castejon, K.B. Wiberg, J. Phys. Chem. 100, 16162 (1996).

${ }^{28}$ A. Karpfen, C.H. Choi, M. Kertesz, J. Phys. Chem. A 101, 7426 (1997).

${ }^{29}$ W.A. Herrebout, B.J. van der Veken, A. Wang, J.R. Durig, J. Phys. Chem. 99, 578 (1995).

${ }^{30}$ R.A. Buckingham, Proc. Royal Soc. London 167, A264 (1938).

${ }^{31}$ Strictly speaking, $P$ should be a polynomial in $r$ (see ref. 30 and references therein). Even for the case of constant $P$, the interaction is between a molecule and a plane, and the expression would have to be integrated to yield a linear function times an exponential. The latter, however, does not improve much the fit in figure 3.4, and we just want a first approximation to the behavior of the rate with pore size. Thus, we keep the simpler equation (3.3.1) in our derivation, and identify $r$ with the pore width. 
${ }^{32}$ For TST to be a good approximation, the activation barrier $\Delta E^{\ddagger}$ for the reaction should be large compared to the thermal energy $R T$. A rule of thumb is that $\Delta E^{\ddagger} / R T$ should be around 5 or greater. For the case of the bulk reaction and the largest pore, this means that TST would not be a very good approximation, especially at the higher temperatures, and dynamic corrections to the rate constant would be important. These corrections are not expected, however, to be so large as to change our conclusions about the shape catalytic effect. In fact, since figures 3 and 4 are on a single and double-log scale, the changes to the plot would be very small. For the smaller pores, the barrier increases so much that TST is expected to be a good approximation.

${ }^{33}$ S. Kondo, E. Hirota, Y. Morino, J. Mol. Spectrosc. 28, 471 (1968).

${ }^{34}$ R. Engeln, D. Consalvo, J. Reuss, Chem. Phys. 160, 427 (1992).

${ }^{35}$ J.C. Sancho-García, A.J. Pérez-Jiménez, J.M. Pérez-Jordá, F. Moscardó, Mol. Phys. 99, 47 (2001).

${ }^{36}$ J.C. Sancho-García, A.J. Pérez-Jiménez, F. Moscardó, J. Phys. Chem. A 105, 11541 (2001).

${ }^{37}$ C.H. Choi, M. Kertesz, A. Karpfen, Chem. Phys. Lett. 276, 266 (1997).

${ }^{38}$ J.C. Sancho-García and A. Karpfen, Theor. Chem. Acc. 115, 427 (2006).

${ }^{39}$ C.H. Choi, M. Kertesz, S. Dobrin, J. Michl, Theor. Chem. Acc. 102, 196 (1999).

${ }^{40}$ M. Kofranek, A. Karpfen, H. Lischka, Chem. Phys. Lett. 189, 281 (1992). 


\section{Chapter 4}

\section{Unimolecular Decomposition of Formaldehyde in Porous Carbons}

\subsection{Introduction}

One of the factors that can influence chemical reactions in confinement is the effect of physical interactions (e.g. dispersion or electrostatic interactions) on both the reaction dynamics and the reaction equilibrium. Materials adsorbed on surfaces, or confined in porous spaces, often have thermodynamic properties that differ markedly from those of the bulk material, since the molecule-surface interactions in such systems are often as important or more so than the molecule-molecule interactions. Since the chemical reaction equilibrium is determined by the thermodynamic properties of the reactants and products involved, such interactions can often displace significantly the equilibrium in the adsorbed phase compared to that in the bulk. These effects are similar to those observed for reactions in solution, and the term "pseudo-solvent effect" has been used to describe them for this reason ${ }^{1}$. Some recent examples in the literature studying the effect of confinement on equilibrium distributions due to physical interactions with the confining material can be found in refs. 1-13.

Although the reaction equilibrium is the most obviously influenced by physical interactions with the confining material, it is also possible for such interactions to influence the reaction dynamics ${ }^{1-5,7}$. For example, if a reaction involves the transference of charge from one atom to another or across a bonding network, the presence of a polarizable environment can enhance this process and lower the reaction barrier. 
With the purpose of studying in detail an effect of the kind described above, in this chapter we focus on the effect of physical interactions with the confining material on the unimolecular decomposition of formaldehyde within carbon pores. This reaction involves the breaking and formation of chemical bonds and a redistribution of electric charge on the atoms involved. We show that interactions with the confining material result in a slight lowering of the barrier for the dissociation. We also show that for large pores, modeled in this work as a single graphene sheet, there is also a shift of equilibrium towards the dissociated species. This trend is reversed in small pores.

The rest of this chapter is organized as follows. In section 4.2 we describe the methods we used to model the unimolecular decomposition of formaldehyde in the gas phase, on a graphene surface, and within carbon slit pores of various pore widths. In section 4.3 we present our results and discuss the influence that the interactions between the molecular species involved and the confining material have on the reaction mechanism and equilibrium. Finally, some concluding remarks are given in section 4.4. The material in this paper has been published in refs. 2-5 and 14 .

\subsection{Methods}

The results presented in this chapter were obtained using plane-wave pseudopotential density functional theory ${ }^{15,16}$ (DFT), with the Becke-Lee-Yang-Parr (BLYP) exchangecorrelation functional $^{17,18}$. Some of the calculations were repeated using the Perdew-BeckeErnzerhof (PBE) exchange-correlation functional ${ }^{19}$ in order to verify that the trends found were not an artifact of the particular choice of exchange-correlation functional. We used ultrasoft pseudopotentials ${ }^{20}$ with a plane-wave cutoff of $35 \mathrm{Ry}$ and a density cutoff of 280 Ry. The reaction pathways both in the gas phase and in confinement were obtained using the Nudged Elastic Band (NEB) method ${ }^{21,22}$ with the climbing image modification ${ }^{23}$ to 
obtain the structure of the transition states and the activation barrier, as implemented in the “Quantum-ESPRESSO” (PWSCF) package ${ }^{24}$. We studied the dissociation reaction $\mathrm{HCOH} \rightarrow \mathrm{CO}+\mathrm{H}_{2}$ in three different environments: (1) In the gas phase, (2) on top of a graphene sheet, and (3) between two graphene sheets separated by distances between 11 au (5.82 $\AA$ ) and 14 au (7.41 $\AA$ ). The first calculation serves as a comparison point and to gauge the accuracy of our results. The second calculation represents the reaction happening in carbon pores that are large compared to the molecular size, and the third set of calculations are a model for the reaction happening within a nanoporous material. The graphene walls were modeled as 32-atom sheets on a hexagonal unit cell with periodic boundary conditions. The cell dimensions were allowed to relax fully before the calculation, yielding an equilibrium carbon-carbon bond length of $1.43 \AA$. In order to maintain a constant pore size in the nanopore calculations, we constrained two of the atoms on the graphene sheets to remain separated by the desired pore width.

In addition to the reaction mechanism and reaction barriers, we obtained the equilibrium distribution of reactants and products in the ideal gas limit for the gas-phase reaction, and the Henry's law limit for the reactions on the surface and the pore, for three cases: the bulk reaction, the reaction above a graphene sheet, and the reaction happening in a pore of width 11 au (as a representative case). In order to do so, we ran phonon calculations for both reactants and products, and calculated the ideal-gas partition function as described in ref. 25. For the adsorbed states, the translational and rotational modes were taken as those of a 2-dimensional ideal gas. In all cases we treated rotations, translations and vibrations as independent of each other. Rotations were treated quantum mechanically at low temperatures. For formaldehyde, since it is an asymmetric top, an exact quantum mechanical expression for the rotational partition function is not available, so we used a perturbation correction of order $O\left(\hbar^{2}\right) \cdot{ }^{26}$ 


\subsection{Results and Discussion}

The energetics for the dissociation of formaldehyde in the gas phase, as obtained from a NEB calculation with 5 images, is shown in table 4.1, as well as the relevant geometric parameters of all images. The last image corresponds to $\mathrm{CO}$ and $\mathrm{H}_{2}$ infinitely far from each other. The activation barrier as predicted by DFT-BLYP is $80.3 \mathrm{kcal} / \mathrm{mol}$, in good agreement with the experimental value of $79.2 \pm 0.8 \mathrm{kcal} / \mathrm{mol}^{27,28}$ and other theoretical results $^{29-40}$. The predicted geometries are also in good agreement with other theoretical calculations. The products, $\mathrm{CO}$ and $\mathrm{H}_{2}$, are higher in energy than formaldehyde by 7.1 $\mathrm{kcal} / \mathrm{mol}$. The standard Gibbs free energy of reaction at $298 \mathrm{~K}$, as obtained from these energies and the phonons obtained from the DFT calculations, is $-5.63 \mathrm{kcal} / \mathrm{mol}$, which is within $1 \mathrm{kcal} / \mathrm{mol}$ of the experimental value of $-6.50 \mathrm{kcal} / \mathrm{mol}^{41}$.

Table 4.1. Optimized geometries and energies of the stationary points along the reaction path for the dissociation of formaldehyde in vacuum. Energies are in $\mathrm{kcal} / \mathrm{mol}$, distances in $\AA$ and angles in degrees.

\begin{tabular}{ccccccc}
\hline Image & Energy & $d_{\mathrm{C}-\mathrm{O}}(\AA)$ & $d_{\mathrm{H}_{1}-\mathrm{H}_{2}}(\AA)$ & $d_{\mathrm{C}-\mathrm{H}_{1}}(\AA)$ & $\alpha_{\mathrm{O}-\mathrm{C}-\mathrm{H}_{1}}\left({ }^{\circ}\right)$ & $\alpha_{\mathrm{H}_{1}-\mathrm{C}-\mathrm{H}_{2}}\left({ }^{\circ}\right)$ \\
\hline $\mathrm{HCOH}$ & 0.0 (ref.) & 1.215 & 1.894 & 1.116 & 122.0 & 116.1 \\
$\mathrm{TS}$ & 80.3 & 1.173 & 1.241 & 1.133 & 152.6 & 43.8 \\
$\mathrm{CO}+\mathrm{H}_{2}$ & 7.1 & 1.140 & 0.755 & - & - & - \\
\hline
\end{tabular}

Figure 4.1 shows the energetics for the dissociation of formaldehyde above a graphene sheet, and within a nanopore of width 11 au (5.82 Å), as obtained from NEB calculations with 11 images. The relevant geometric parameters and the energies are also shown in table 4.2. 

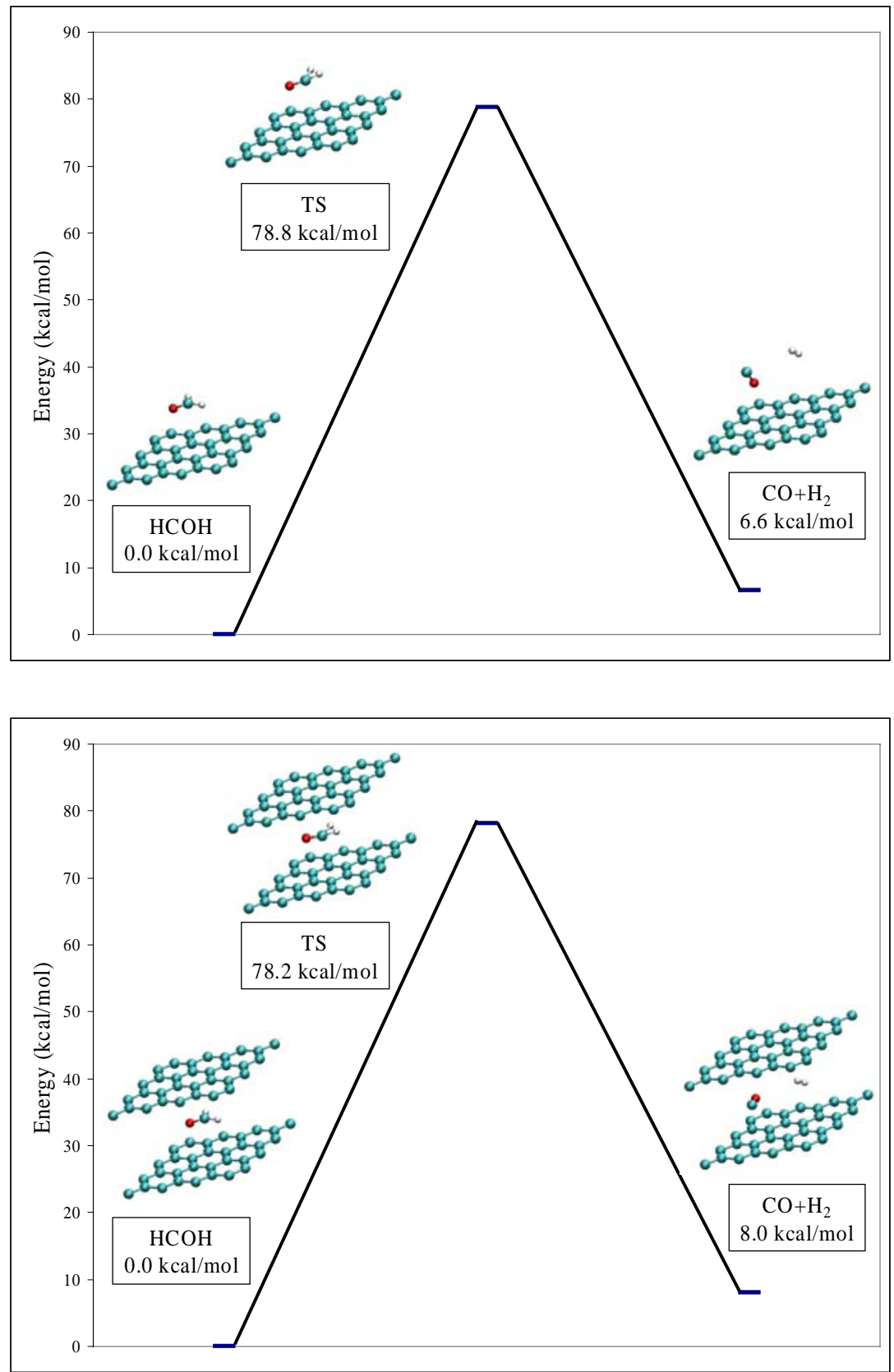

Figure 4.1. Energetics for the dissociation of formaldehyde over a graphene sheet (top) and within a 11 au (5.82 Å) wide pore (bottom). 
The activation barrier for the dissociation over a graphene sheet is lower than that in the bulk by about $1.5 \mathrm{kcal} / \mathrm{mol}$, and it is further lowered by another $0.6 \mathrm{kcal} / \mathrm{mol}$ when the reaction happens within the 11 au-wide pore. The reduction of the activation barrier could be explained by the large polarizability of the graphene walls, which thus acts in a way similar to a solvent with a high dielectric constant. Since the dissociation requires charge to travel along the $\mathrm{C}-\mathrm{O}$ bond, the highly polarizable medium helps the reaction to happen. Halls and Schlegel ${ }^{1}$ have reported a similar effect for a different reaction happening within a carbon nanotube. The effect in this case is, however, small in relative terms due to the large energy barrier for the dissociation.

The equilibrium is, unlike the activation barrier, shifted in different directions above the graphene sheet and in the pore. When the reaction happens above a single graphene surface, the products of the reaction $\left(\mathrm{CO}\right.$ and $\left.\mathrm{H}_{2}\right)$ are $6.6 \mathrm{kcal} / \mathrm{mol}$ above the reactants, which is $0.5 \mathrm{kcal} / \mathrm{mol}$ lower than the same value for the bulk reaction. For the reaction happening within the 11 au pore, however, the products are $8.0 \mathrm{kcal} / \mathrm{mol}$ above the reactants, which represents an increase of almost $1 \mathrm{kcal} / \mathrm{mol}$ with respect to the bulk value. The lower energy of the products for the reaction happening on the sheet means that the products are stabilized by physisorption on the surface slightly more than formaldehyde ${ }^{42}$. For the narrow pore, however, the trend is different because the steric hindrance affects the products in a way different from the way it affects the reactant - $\mathrm{CO}$ and $\mathrm{H}_{2}$ adsorb at an angle above the sheet, whereas formaldehyde adsorbs parallel to the surface. Thus the reduction of the pore size makes the energy of the products increase faster than the energy of the reactant.

The effect of pore size on the activation barrier for the dissociation and the energy difference between the reactants and the products is shown in table 4.3. 
Table 4.2. Optimized geometries and energies of the stationary points along the reaction path for the dissociation of formaldehyde above a graphene sheet (a) and within a slit pore of width 11 au (b), as obtained from NEB calculations. The differences with the values for the bulk reaction are given in parentheses for comparison. Energies are in $\mathrm{kcal} / \mathrm{mol}$, distances in $\AA$ and angles in degrees.

(a)

\begin{tabular}{|c|c|c|c|c|c|c|}
\hline Image & Energy & $d_{\mathrm{C}-\mathrm{O}}(\AA)$ & $d_{\mathrm{H}_{1}-\mathrm{H}_{2}}(\AA)$ & $d_{\mathrm{C}-\mathrm{H}_{1}}(\AA)$ & $\alpha_{\mathrm{O}-\mathrm{C}-\mathrm{H}_{1}}\left({ }^{\circ}\right)$ & $\begin{array}{c}\alpha_{\mathrm{H}_{1}-\mathrm{C}-\mathrm{H}_{2}} \\
\left(^{\mathrm{o}}\right)\end{array}$ \\
\hline \multirow{2}{*}{$\mathrm{HCOH}$} & 0.0 & 1.216 & 1.894 & 1.116 & 122.0 & 116.1 \\
\hline & (ref.) & $(+0.001)$ & $(0)$ & $(0)$ & $(0)$ & $(0)$ \\
\hline \multirow{2}{*}{ TS } & 78.8 & 1.177 & 1.317 & 1.101 & 163.5 & 51.5 \\
\hline & $(-1.5)$ & $(+0.004)$ & $(+0.076)$ & $(-0.032)$ & $(+10.9)$ & $(+7.7)$ \\
\hline \multirow{2}{*}{$\mathrm{CO}+\mathrm{H}_{2}$} & 6.6 & 1.141 & 0.755 & \multirow{2}{*}{-} & \multirow{2}{*}{-} & \multirow{2}{*}{ - } \\
\hline & $(-0.5)$ & $(+0.001)$ & $(0)$ & & & \\
\hline \multicolumn{7}{|c|}{ (b) } \\
\hline Image & Energy & $d_{\mathrm{C}-\mathrm{O}}(\AA)$ & $d_{\mathrm{H}_{1}-\mathrm{H}_{2}}(\AA)$ & $d_{\mathrm{C}-\mathrm{H}_{1}}(\AA)$ & $\alpha_{\mathrm{O}-\mathrm{C}-\mathrm{H}_{1}}\left({ }^{\circ}\right)$ & $\alpha_{\mathrm{H}_{1}-\mathrm{C}-\mathrm{H}_{2}}\left({ }^{\circ}\right)$ \\
\hline \multirow{2}{*}{$\mathrm{HCOH}$} & 0.0 & 1.219 & 1.886 & 1.113 & 122.1 & 115.8 \\
\hline & (ref.) & $(+0.004)$ & $(-0.008)$ & $(-0.003)$ & $(+0.1)$ & $(-0.3)$ \\
\hline \multirow{2}{*}{$\mathrm{TS}$} & 78.2 & 1.174 & 1.358 & 1.095 & 164.7 & 51.4 \\
\hline & $(-2.1)$ & $(+0.001)$ & $(+0.117)$ & $(-0.038)$ & $(+12.1)$ & $(+7.6)$ \\
\hline \multirow{2}{*}{$\mathrm{CO}+\mathrm{H}_{2}$} & 8.0 & 1.143 & 0.753 & \multirow[b]{2}{*}{ - } & \multirow{2}{*}{-} & \multirow[b]{2}{*}{-} \\
\hline & $(+0.9)$ & $(+0.003)$ & $(-0.002)$ & & & \\
\hline
\end{tabular}

The results in table 4.3 show that the slight reduction of the reaction barrier increases with decreasing pore size, even for very narrow pores. This is related to the fact that both formaldehyde and the transition state are planar, and thus the steric hindrance increases their energy in a similar way. The electrostatic interaction that lowers the reaction barrier, 
however, is stronger the closer the molecule is to the pore walls, resulting in a reduction of the barrier at all distances. The energy difference between reactants and products at the smaller pore sizes does not, however, change appreciably for the pore widths studied. For all these pore sizes the products, $\mathrm{CO}$ and $\mathrm{H}_{2}$, adsorb parallel to the carbon walls, as opposed to what happens above a single graphene sheet. Thus the steric hindrance increases the energies of both the reactant and the products in a similar way with changing pore sizes, and the net effect on the energy of reaction is negligible. There is most likely a transition between the planar conformation of the products above the surface and the inclined one seen above a single graphene sheet, but this transition would occur at pore sizes greater than those studied in this work.

Table 4.3. Activation barriers and energies of reaction for the dissociation of formaldehyde within a carbon pore as a function of pore size, as obtained from NEB calculations. The values for the dissociation above a graphene sheet correspond to an infinite pore width. The differences with respect to the bulk reaction are given in parentheses for comparison. All the energies are in $\mathrm{kcal} / \mathrm{mol}$.

\begin{tabular}{cccccc}
\hline Pore width & $\begin{array}{c}14 \mathrm{au} \\
\text { (sheet) }\end{array}$ & $\begin{array}{c}13 \mathrm{au} \\
(7.41 \AA)\end{array}$ & $\begin{array}{c}12 \mathrm{au} \\
(6.88 \AA)\end{array}$ & $\begin{array}{c}11 \mathrm{au} \\
(6.35 \AA)\end{array}$ & $(5.82 \AA)$ \\
\hline$E_{\mathrm{TS}}-E_{\mathrm{HCOH}}$ & $78.8(-1.5)$ & $78.6(-1.7)$ & $78.3(-2.0)$ & $78.2(-2.1)$ & $77.7(-2.6)$ \\
$E_{\mathrm{CO}+\mathrm{H}_{2}}-E_{\mathrm{HCOH}}$ & $6.6(-0.5)$ & $8.1(+1.0)$ & $8.1(+1.0)$ & $8.1(+1.0)$ & $8.0(+0.9)$ \\
\hline
\end{tabular}

In order to quantify the effect of the interactions between the reacting mixture and the carbon pores on the reaction equilibrium at finite temperatures, we computed the equilibrium constant based on mole fractions, $K_{x}=x_{\mathrm{HCOH}} /\left(x_{\mathrm{CO}} x_{\mathrm{H}_{2}}\right)$, as a function of temperature for the bulk, sheet, and 11 au pore. The values for the bulk were calculated assuming an initial amount of 1 mole of formaldehyde at the standard pressure $P^{0}=1 \mathrm{bar}$. 
The values for the sheet and the pore were obtained by imposing the conditions of chemical and thermal equilibrium with the corresponding bulk mixture. The results are presented in Figure 4.2, which shows a plot of $\ln \left(K_{x}\right)$ vs. reciprocal temperature. At lower temperatures, the equilibrium distributions show the trend one would expect from the values at 0 Kelvin, i.e. the equilibrium is displaced towards the products the most (highest $K_{x}$ ) over the graphene sheet, and the least (lowest $K_{x}$ ) for the 11 au pore, with the bulk in between the two. However, since the reaction is most endothermic within the 11 au pore, it follows from van't Hoff's law that the equilibrium constant grows fastest with temperature for this system. Similarly, for the reaction happening over the graphene sheet, the equilibrium constant has the slowest increase with temperature, with the bulk reaction in between the two. Thus the trend in the equilibrium distributions is reversed at high temperatures.

In table 4.4, we compare the equilibrium distributions (in terms of $K_{x}$ ) for the reaction happening in a bulk ideal gas, over a graphene sheet, and within the graphite pore of width $11 \mathrm{au}$. At low temperatures (below $\sim 73.0 \mathrm{~K}$ ), the equilibrium is most displaced toward the formation of $\mathrm{CO}+\mathrm{H}_{2}$ for the reaction happening on top of a graphene sheet, with the pore being the most displaced toward formaldehyde. Between approximately $73.0 \mathrm{~K}$ and 82.9 $\mathrm{K}$, the bulk reaction is the one most displaced toward formaldehyde, with the pore being intermediate between the sheet and the bulk. Then, between $\sim 82.9 \mathrm{~K}$ and $\sim 555.0 \mathrm{~K}$, the reaction is most displaced toward $\mathrm{CO}+\mathrm{H}_{2}$ within the narrow pore, and most displaced toward $\mathrm{CH}_{2} \mathrm{O}$ in the bulk ideal gas. Finally, for high temperatures (above $\sim 555.0 \mathrm{~K}$ ), the equilibrium is most displaced toward $\mathrm{CO}+\mathrm{H}_{2}$ within the narrow pore, and most displaced toward formaldehyde above the graphene surface. As mentioned in the previous paragraph, these trends are to be expected considering that the reaction is most endothermic within the narrow pore and least endothermic on top of the graphene sheet. 


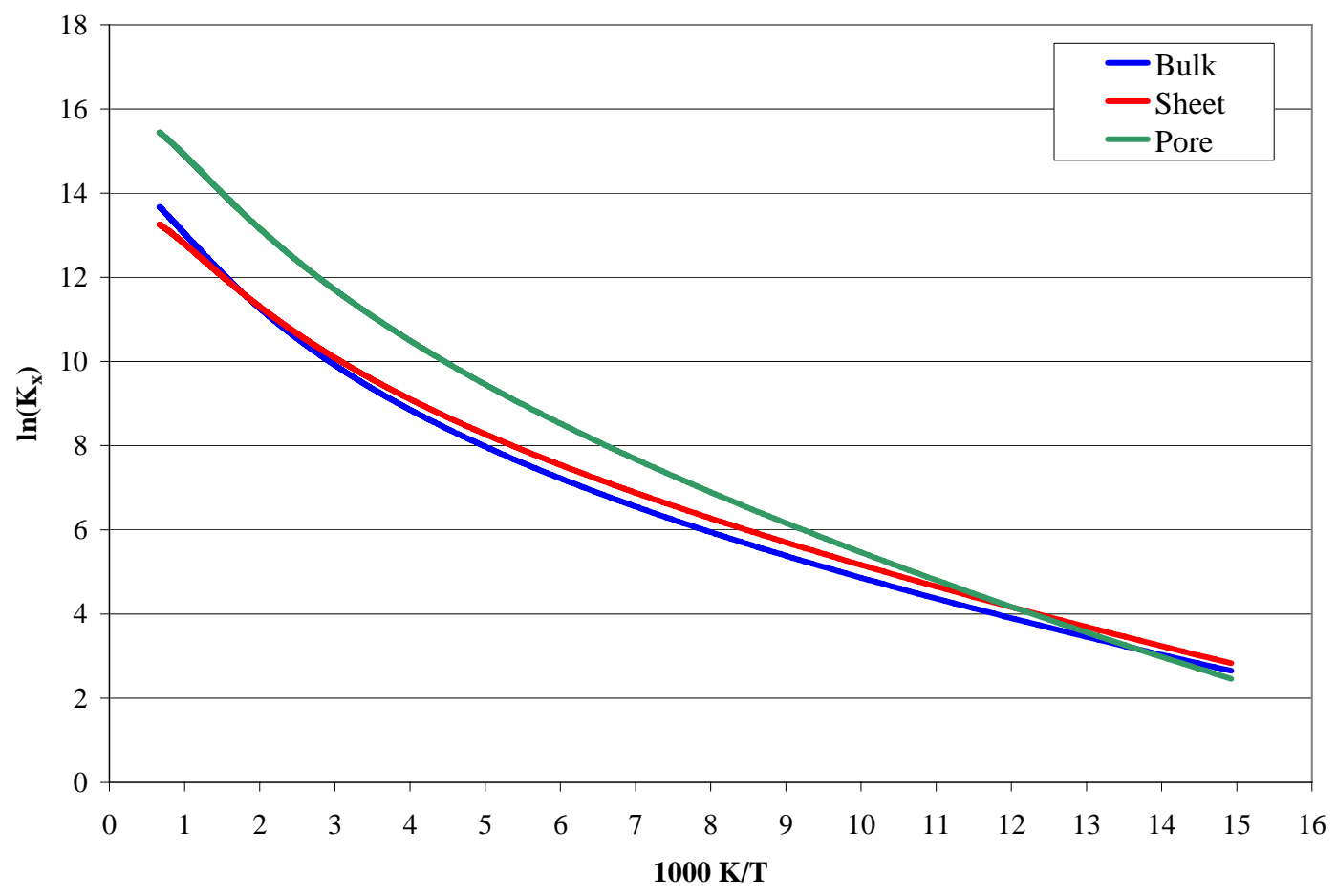

Figure 4.2. A van't Hoff plot comparing the equilibrium distribution of reactants and products for the decomposition of formaldehyde happening in a bulk ideal gas, above a graphene sheet, and within a graphite slit pore of width 11 au.

Table 4.4. Comparison between the equilibrium distributions of reactants and products in the bulk ideal gas, above a graphene sheet, and within a 11 au graphite slit pore for different temperature intervals.

\begin{tabular}{cccc}
\hline Temperature range $(\mathrm{K})$ & Lowest $K_{x}$ & Intermediate $K_{x}$ & Highest $K_{x}$ \\
\hline $0-73.0$ & Pore & Bulk & Sheet \\
$73.0-82.9$ & Bulk & Pore & Sheet \\
$82.9-555.0$ & Bulk & Sheet & Pore \\
$>555.0$ & Sheet & Bulk & Pore \\
\hline
\end{tabular}




\subsection{Concluding Remarks}

We have shown, using first principles calculations, that both the kinetics and the chemical equilibrium for the unimolecular decomposition of formaldehyde are affected by confinement within carbon pores. The activation barrier for the reaction is slightly lowered by the presence of a single graphene surface, and is further lowered within a narrow pore. We believe this trend to be due to a pseudo-solvent effect similar to the one observed in ref. 1.

The endothermicity of the reaction is modified in different directions depending on pore size, being lower than that of the bulk ideal gas for large pores (or a single carbon surface), and higher for narrow pores. This difference appears to be due to a transition between the products being adsorbed perpendicular to the carbon surface and the products being adsorbed parallel to it. This transition is due to the additional steric hindrance at lower pore sizes.

Because of the difference in endothermicities, the equilibrium distribution of reactants and products is altered in different ways by the confining material depending on temperature. Thus for temperatures close to $0 \mathrm{~K}$, the trend towards the formation of $\mathrm{CO}+\mathrm{H}_{2}$ goes in the order Pore $<$ Bulk $<$ Sheet This trend then changes, in order of increasing temperature, to Bulk $<$ Pore $<$ Sheet, then Bulk $<$ Sheet $<$ Pore, and finally Sheet $<$ Bulk $<$ Pore at high temperatures. This means that the optimal pore size of the confining material to carry out the reaction, in terms of equilibrium, would be a function of the operating temperature. 


\section{References and Notes}

${ }^{1}$ M.D. Halls, H.B. Schlegel, J. Phys. Chem. B 106, 1921 (2002) .

${ }^{2}$ E. E. Santiso, M.K. Kostov, A.M. George, M.B. Nardelli, K.E. Gubbins, Appl. Surf. Sci., 253, 5570 (2007).

${ }^{3}$ E.E. Santiso, A.M. George, K.E. Gubbins, M.B. Nardelli, J. Chem. Phys. 125, 084711 (2006)

${ }^{4}$ E.E. Santiso, A.M. George, C.H. Turner, M.K. Kostov, K.E. Gubbins, M. Buongiorno-Nardelli, M.

Sliwinska-Bartkowiak, Appl. Surf. Sci. 252, 766 (2005).

${ }^{5}$ E.E. Santiso, A.M. George, M. Sliwinska-Bartkowiak, M. Buongiorno-Nardelli, K.E. Gubbins, Adsorption 11, 349 (2005).

${ }^{6}$ C.H. Turner, K.E. Gubbins, J. Chem. Phys. 119, 6057 (2003).

${ }^{7}$ C.H. Turner, J.K. Brennan, J.K. Johnson, K.E. Gubbins, J. Chem. Phys. 116, 2138 (2002).

${ }^{8}$ C.H. Turner, J. Pikunic, K.E. Gubbins, Mol. Phys. 99, 1991 (2001).

${ }^{9}$ C.H. Turner, J.K. Johnson, K.E. Gubbins, J. Chem Phys. 114, 1851 (2001).

${ }^{10}$ M. Lisal, J.K. Brennan, W.R. Smith, J. Chem. Phys. 124, 064712 (2006).

${ }^{11}$ O. Byl, P. Kondratyuk, J.T. Yates, J. Phys. Chem. B 107, 4277 (2003).

${ }^{12}$ K. Kaneko, N. Fukuzaki, K. Kakei, T. Suzuki, S. Ozeki, Langmuir 5, 960 (1989).

${ }^{13}$ J. Imai, M. Souma, S. Ozeki, T. Suzuki, K. Kaneko, J. Phys. Chem. 95, 9955 (1991).

${ }^{14}$ E.E. Santiso, L. Huang, K.E. Gubbins, M.K. Kostov, A.M. George and Marco Buongiorno Nardelli, “Ab initio simulations of chemical reactions in nanostructured carbon materials”, in Quantum Chemical Calculations of Surfaces and Interfaces of Materials, American Scientific Publishers, Valencia, California (2007).

${ }^{15}$ R.G. Parr, W. Yang, Density-Functional Theory of Atoms and Molecules, Oxford University Press, Oxford (1989).

${ }^{16}$ E.E. Santiso, K.E. Gubbins, Mol. Simul. 30, 699 (2004).

${ }^{17}$ A.D. Becke, Phys. Rev. A 38, 3098 (1988). 
${ }^{18}$ C. Lee, W. Yang, R.G. Parr, Phys. Rev. B 37, 785 (1988).

${ }^{19}$ J.P. Perdew, K. Burke, M. Ernzerhof, Phys. Rev. Lett. 77, 3865 (1996).

${ }^{20}$ D. Vanderbilt, Phys. Rev. B 41, 7892 (1990).

${ }^{21}$ G. Mills, H. Jónsson, G.K. Schenter, Surf. Sci. 324, 305 (1995).

${ }^{22}$ H. Jónsson, G. Mills, K.W. Jacobsen, in Classical and Quantum Dynamics in Condensed Phase

Simulations, B.J. Berne, G. Ciccotti, D.F. Coker, Eds., World Scientific, Singapore (1998).

${ }^{23}$ G. Henkelman, B.P. Uberuaga, H. Jónsson, J. Chem. Phys. 113, 9901 (2000).

${ }^{24}$ S. Baroni, A. Dal Corso, S. de Gironcoli, P. Giannozzi, C. Cavazzoni, G. Ballabio, S. Scandolo, G.

Chiarotti, P. Focher, A. Pasquarello, K. Laasonen, A. Trave, R. Car, N. Marzari, A. Kokalj, http://www.pwscf.org/.

${ }^{25}$ T.M. Reed, K.E. Gubbins, Applied Statistical Mechanics, Butterworth-Heinemann, Boston (1973).

${ }^{26}$ C.G. Gray, K.E. Gubbins, Theory of Molecular Fluids, Clarendon Press, Oxford (1984).

${ }^{27}$ W.F. Polik, D.R. Guyer, C.B. Moore, J. Chem. Phys. 92, 3453 (1990).

${ }^{28}$ W.H. Green, Jr., C.B. Moore, W.F. Polik, Annu. Rev. Phys. Chem. 43, 591 (1992).

${ }^{29}$ Y.-T. Chang, C. Minichino, W.H. Miller, J. Chem. Phys. 96, 4341 (1992).

${ }^{30}$ L. Deng, T. Ziegler, L. Fan, J. Chem. Phys. 99, 3823 (1993).

${ }^{31}$ W. Chen, W.L. Hase, H.B. Schlegel, Chem. Phys. Lett. 228, 436 (1994).

${ }^{32}$ H. Nakano, K. Nakayama, K. Hirao, M. Dupuis, J. Chem. Phys. 106, 4912 (1997).

${ }^{33}$ P.J. Bruna, M.R.J. Hachey, F. Grein, J. Mol. Struct. (Theochem) 400, 177 (1997).

${ }^{34}$ D. Feller, M. Dupuis, B.C. Garrett, J. Chem. Phys. 113, 218 (2000).

${ }^{35}$ X. Li, J.M. Millam, H.B. Schlegel, J. Chem. Phys. 113, 10062 (2000).

${ }^{36}$ G.F. Bauerfeldt, L.M.M. de Albuquerque, G. Arbilla, E.C. da Silva, J. Mol. Struct. (Theochem) 580, 147 (2002).

${ }^{37}$ T. Yonehara, S. Kato, J. Chem. Phys. 117, 11131 (2002).

${ }^{38}$ X. Zhang, S. Zou, L.B. Harding, J.M. Bowman, J. Phys. Chem. A 108, 8980 (2004). 
39 J.L. Rheinecker, X. Zhang, J.M. Bowman, Mol. Phys. 103, 1067 (2005).

${ }^{40}$ S. Maeda, K. Ohno, J. Phys. Chem. A 109, 5742 (2005).

${ }^{41}$ NIST Standard Reference Database Number 69, June 2005 Release, http://webbook.nist.gov/chemistry/.

${ }^{42}$ DFT-BLYP does not predict an interaction between $\mathrm{H}_{2}$ and a graphene sheet except at very short distances.

In the present case, however, they interact because of the polarization of the graphene sheet induced by the CO molecule. Their interaction in this case is a 3-body effect. 


\section{Chapter 5}

\section{Dissociation of Water on Defective Carbon Surfaces}

\subsection{Introduction}

The third class of factors that can influence chemical reactions in confinement, as discussed in Chapter 1, is the interactions that involve a significant rearrangement of the electron clouds of the confined molecule, including the formation of chemical bonds with the surface. In the previous examples considered (Chapters 3 and 4), we studied molecules interacting with perfect graphene surfaces, which is chemically inert. There are, however, several ways to "turn on" the chemical activity of graphene, including the creation of defects in the carbon structure ${ }^{1,2}$, doping ${ }^{3}$ and the addition of functional groups ${ }^{3}$. In this chapter we consider a chemical reaction - the thermal splitting of water - happening over a defective carbon surface, and show how the chemical interactions with the defect give rise to a chemistry completely different from that seen in the bulk. In addition, we consider the possibility of exploiting changes in the geometry of the surface (see Chapter 3), to further displace the reaction in the desired direction.

The direct thermal splitting of water, $\mathrm{H}_{2} \mathrm{O} \rightarrow \mathrm{H}_{2}+1 / 2 \mathrm{O}_{2}$, which does not generate $\mathrm{CO}_{2}$ and produces highly pure hydrogen, requires temperatures in excess of $2000{ }^{\circ} \mathrm{C}$, thus making its practical implementation very challenging ${ }^{4}$. In addition, it has a relatively low hydrogen yield of about $25 \%$. The fundamental limitation of the thermal splitting of bulk water is the fact that the ground state of oxygen is paramagnetic (a spin triplet), whereas the ground state of water is diamagnetic (a spin singlet). Breaking a water molecule thus requires an excess of $45 \mathrm{kcal} / \mathrm{mol}$ of additional energy to excite the molecule to a higher spin state 
prior to the dissociation. This energy is later released as unrecovered heat when oxygen relaxes to its ground state. Although solutions for exciting the water molecule to the dissociative spin-triplet state have been proposed (photo-excitation, enzymatic and biochemical reactions, etc.), they still fall short of an industrially competitive production process. In this work, we propose an alternative paradigm in $\mathrm{H}_{2}$ production: a process in which the system remains on the singlet spin surface throughout the reaction by exploiting the reactivity of defects in carbon materials.

Graphitic carbon substrates such as activated carbon fibers, nanotubes and fullerenes have been extensively studied for their potential as media for energy storage and $\mathrm{H}_{2}$ sequestration $^{6,7,8}$. However, there is no report of using them as chemical reactors rather than fuel tanks. Using $a b$ initio Density Functional Theory ${ }^{9,10}$ (DFT) techniques, we have found evidence that if structural defects are present or are artificially created in graphiticlike materials, a rich chemistry emerges, yielding many possible water dissociation pathways to produce hydrogen, some of which have activation barriers lower than half the value for the dissociation of bulk water. This means that the production of hydrogen can be achieved at temperatures below $1000^{\circ} \mathrm{C}$.

In the following section, we describe the methods we used to study the dissociation of water over single vacancy defects in graphene and in a model $(10,10)$ nanotube, and discuss our results. The material in this chapter has been published in refs. 11-13.

\subsection{Thermal Splitting of Water on Defective Carbon Surfaces}

In Figure 5.1 we show the estimated equilibrium yield for the dissociation of water on a single vacancy in a nanotube and a graphene sheet as compared with the direct thermolysis 
of the water molecule in free space. It is clear that the defective carbon substrate can produce hydrogen from water at temperatures at least a factor of 2 lower for comparable hydrogen yields ${ }^{14}$.

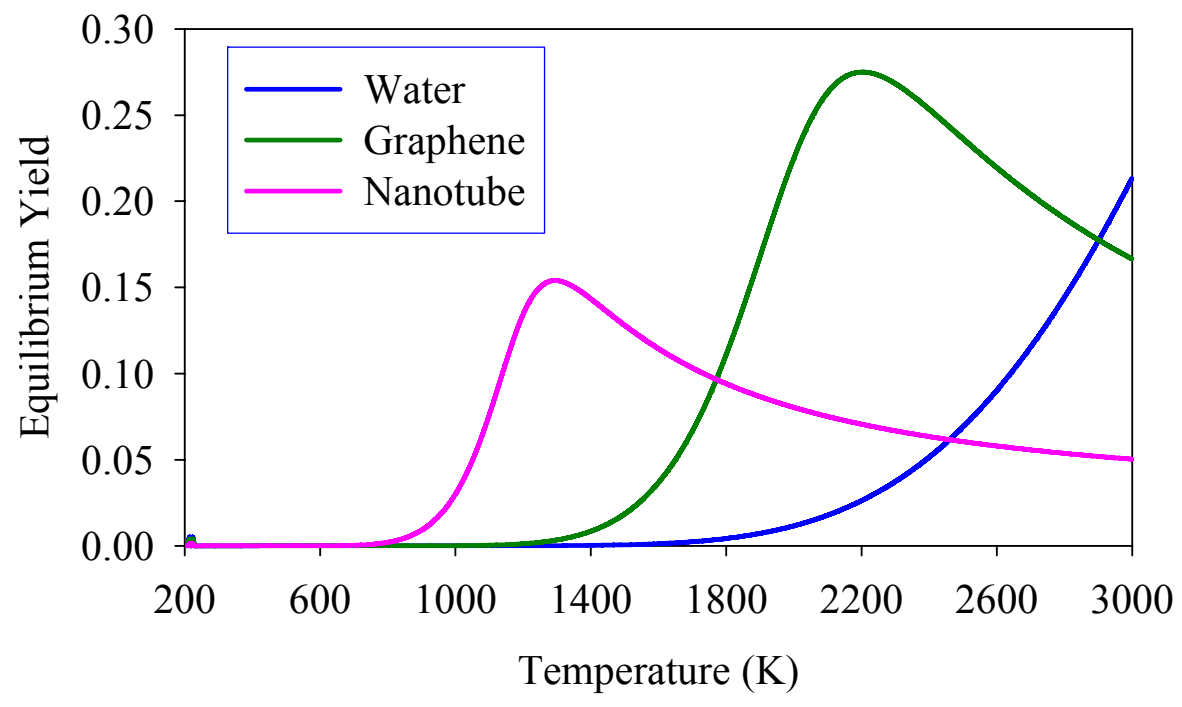

Figure 5.1 Ideal-gas equilibrium yields (fraction of the water that is converted to hydrogen at equilibrium in \% mole) for the dissociation of water in bulk (blue), over a single vacancy in graphene (green) and over a single vacancy in a 10-10 carbon nanotube (pink). The values correspond to a pressure of 1 bar and an initial equimolecular mixture of water and vacancies.

Recent studies have found experimental evidence for the existence of a variety of metastable defects in graphitic carbon materials ${ }^{15}$. These defects can be as-grown or can be induced in both graphene layers and carbon nanotubes by employing electron or ion irradiation ${ }^{16-17}$. Among these defects, mono-vacancies in particular are the most common and they have been experimentally shown to be numerous and stable. Materials containing such defects have potential applications as catalysts, due to the presence of carbon atoms with unsaturated valence shells in their structures. In this work we demonstrate the 
possibility of taking advantage of these vacancy sites to carry out the thermal splitting of water at temperatures substantially lower than $2000^{\circ} \mathrm{C}$ and much higher hydrogen yield.

The electronic structure calculations presented in this work were done using plane-wave density functional theory (DFT) ${ }^{18,19}$ with ultrasoft pseudopotentials ${ }^{20}$ as implemented in the $\mathrm{CPMD}^{21}$ and $\mathrm{PWSCF}^{22}$ codes. We considered both the Becke-Lee-Yang-Parr ${ }^{23,24}$ (BLYP) and Perdew-Becke-Ernzerhof ${ }^{25}$ (PBE) exchange-correlation functionals and found negligible differences in our final results. For graphene we used a periodic super-cell with 32 carbon atoms (see Fig. 5.2), while we simulated the curved nanotube shell as a finite section of 39 carbon atoms with edges saturated with Hydrogen (see Fig. 5.4, H atoms not shown in the picture).

The study of the water splitting on the vacancy requires the knowledge of all possible stable states, transition states and reaction pathways, and the Potential Energy Surface (PES) for this system is quite complicated due to the presence of several "dangling bonds". For this reason, the exploration of the PES has been done using a combination of techniques. First, we identified stable intermediates using the Laio-Parrinello Metadynamics $^{26,27}$ method. This method is a very efficient approach for the exploration of the free energy surface of a reactive system with minimal a priori knowledge of the possible reaction pathways. Given a long enough simulation time, this method allows identifying all the stable states in the PES for the system of choice. The configurations of all the energy minima obtained from Metadynamics were further relaxed with a strict convergence criterion. The structure optimizations for all stable states were carried out for both singlet $(S=0)$ and triplet $(S=1)$ conformations, in order to establish the spin symmetry of the corresponding ground state.

Reaction pathways were obtained using the Nudged Elastic Band ${ }^{28,29}$ (NEB) method of Jónsson et al. with the climbing image modification ${ }^{30}$ in order to determine the structures 
of the transition states. In some cases the structure of the transition state was confirmed by further optimization using the Rational Function Optimization (RFO) method ${ }^{31,32}$. As a test for the accuracy of our approach we have verified that our data for the splitting of a single bulk water molecule agreed with experimental results and other theoretical calculations. ${ }^{33}$ Finally, the standard Gibbs free energy changes for all reactions were computed from the molecular partition functions ${ }^{34}$ and used to obtain the equilibrium yields at different temperatures in the ideal gas limit.

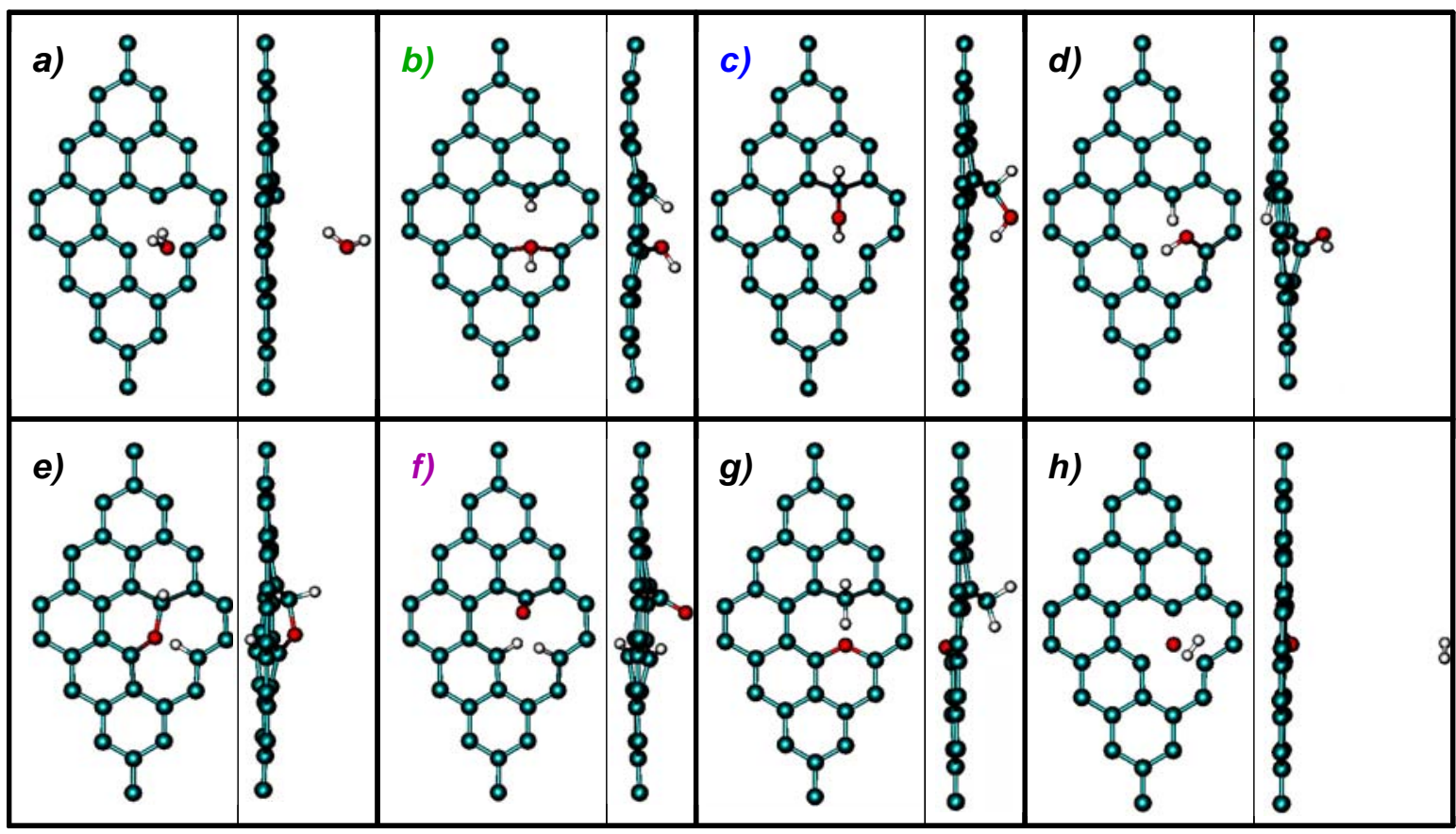

Figure 5.2. Top and side views of various intermediate states in the water splitting reaction over a vacancy site in graphene. (a) initial physisorbed state; (b) IM1: spinsinglet state with the highest energy; (c) IM2: spin-singlet state; (d) IM3: the only spin-triplet state ( $\mathrm{S}=1)$; (e) IM4: spin-singlet state; (f) $K$ (for "ketone"): spin-singlet state; (g) $E$ (for "ether"): a spin-singlet. This is the global minimum of the potential energy surface; (h) F (for "final"): spin-singlet state; the oxygen occupies the vacancy site on the surface and hydrogen is away from it. 
Vacancies in carbon nano-materials indeed have the potential to dissociate water: their formation energy in graphene is approximately $\sim 170 \pm 10 \mathrm{kcal} / \mathrm{mol}^{35,36}$ whereas the reaction enthalpy for the splitting of water is $116 \mathrm{kcal} / \mathrm{mol}^{37}$. The ground state shows a paramagnetic structure where the $D_{3 h}$ symmetric vacancy undergoes a Jahn-Teller distortion, leading to out-of-plane motion of one of the "dangling bond" atoms, (which we denote by $\mathrm{C}_{3}$, see Fig. 5.2a) ${ }^{38,39}$.

In the full exploration of the Potential Energy Surface (PES) for this system, shown in Figure 5.3, we chose the relaxed physisorbed water molecule over the center of the vacancy as the starting ("reactant") point of the reaction and used it as the energy reference in all calculations. We labeled this state by $I$ (for "initial", see Fig. 5.2a). In addition to this initial physisorbed state, we found seven other stable structures that are relevant for the decomposition reaction, including the final state $F$ ("final", Fig. 5.2h) where the hydrogen molecule is fully separated. All the stable structures, including the $I$ and $F$ states are shown in Figure 5.2.

From this knowledge, we investigated all the possible reaction pathways leading to the final state $F$. Figure 5.3 shows the full PES for the reaction while the corresponding energy barriers $\mathrm{E}_{\mathrm{A}}$ are reported in Table 5.1. This complex energy surface exhibits several favorable reaction channels for producing hydrogen. All these reaction pathways are isogyric, i.e. the system stays on the spin singlet surface, which lowers the reaction barrier for dissociation. Among those favorable channels are: $I \rightarrow I M I \rightarrow F$ (with maximum energy barrier $\left.E_{A, \max }=16 \mathrm{kcal} / \mathrm{mol}\right), I \rightarrow K \rightarrow F \quad\left(E_{A, \max }=49 \mathrm{kcal} / \mathrm{mol}\right), I \rightarrow I M 2 \rightarrow F \quad\left(E_{A, \max }=58\right.$ $\mathrm{kcal} / \mathrm{mol})$, and the "straight dissociation" $I \rightarrow F\left(E_{A, \max }=55 \mathrm{kcal} / \mathrm{mol}\right)$. In the straight dissociation reaction we observe a "roaming atom" reaction mechanism - one hydrogen atom explores a large region of the potential energy surface before binding with the second $\mathrm{H}$ atom. Such a reaction mechanism has been just recently discovered in the $\mathrm{HCHO}$ decomposition reaction ${ }^{40}$. 


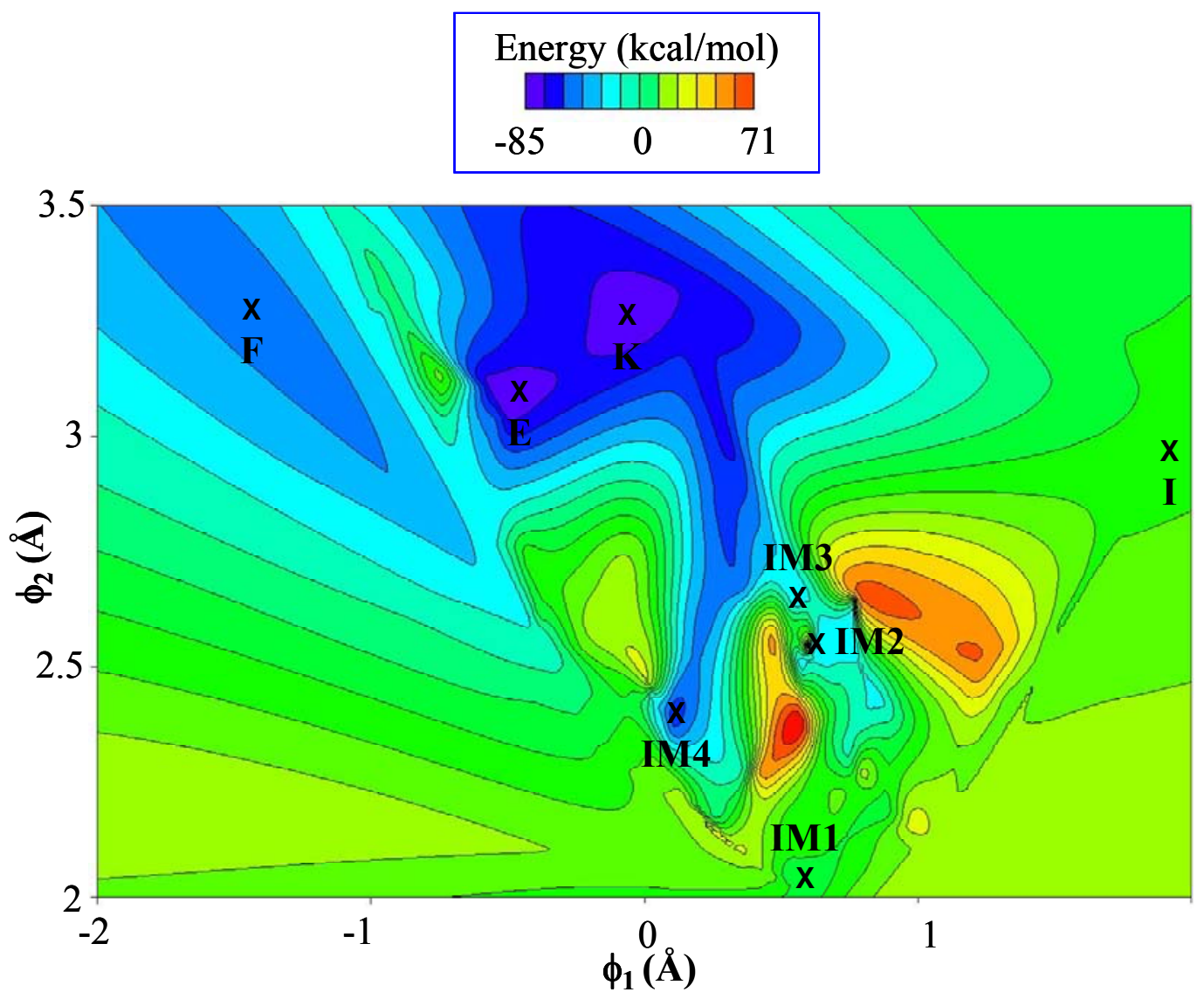

Figure 5.3. Contour plot of the PES for the water dissociation reaction on a vacancy in graphene, as obtained by interpolation of the NEB trajectories. Different configurations are labeled as in Fig. 5.2. The order parameters $\phi_{1}$ and $\phi_{2}$ are defined as $\left.\phi_{1}=(\langle\mathrm{CO}\rangle-\langle\mathrm{OH}\rangle) / \sqrt{ } 2\right)$ and $\left.\phi_{2}=(\langle\mathrm{CO}\rangle+\langle\mathrm{OH}\rangle) / \sqrt{ } 2\right)$, where $\langle\mathrm{CO}\rangle$ is the average distance between the oxygen atom and the carbons immediately adjacent to the vacancy, and $\langle\mathrm{OH}\rangle$ is the average distance between the oxygen atom and the hydrogen atoms.

In reactions involving intermediate states, the process exploits different mechanisms, depending on the species that saturate the $\mathrm{C}_{3}$ "dangling bond" first. In the pathways through $K$ and $I M 4$ states, the $\mathrm{O}$ atom saturates $\mathrm{C}_{3}$ with a very small barrier $(3 \mathrm{kcal} / \mathrm{mol})$, 
whereas going through IMI and IM3 involves the saturation of $\mathrm{C}_{3}$ by $\mathrm{H}$, still with very low energy barriers $(16$ and $15 \mathrm{kcal} / \mathrm{mol})$. In $I M 2$, where both $\mathrm{OH}$ and $\mathrm{H}$ are bonded with $\mathrm{C}_{3}$, the barrier is relatively larger $(33 \mathrm{kcal} / \mathrm{mol})$ but still small compared to the straight dissociation. The number of possible reaction pathways that branch from these intermediate states is large and is best summarized by the activation energy data reported in Table 5.1. An analysis of the data in this table and in Fig. 5.3 reveals that, at moderate temperatures, the most favorable pathway to the $F$ state is $I \rightarrow I M I \rightarrow F$, and the most important competing reactions are the ones toward the $K$ and $E$ states. This is consistent with the equilibrium data shown in Fig. 5.1, where it is seen that at low temperatures, the formation of $E$ and $K$ are most favorable, with the equilibrium shifting towards $F$ at moderate temperatures. None of the other intermediate states are appreciably present. ${ }^{43}$

With the goal of further increasing the efficiency of the dissociation process and the selectivity towards the final dissociated state, we investigated whether the curvature of the reactive substrate, as in a nanotube, could improve upon the reaction mechanism.

In general, the removal of any $\mathrm{C}$ atom in a hexagonal graphite network implies the creation of three two-coordinated $\mathrm{C}$ atoms. When the atomic layer is strongly curved, however, it is possible for two of the "dangling bond" atoms to recombine, thus creating a pentagon. This structure is more stable, since the reconstructed bond is much shorter compared to the distance between two "dangling bond" atoms in graphene $(1.65 \AA$ for a $(10,10)$ tube versus $2.46 \AA$ for graphene). In the nanotube, the second-nearest neighbor distance is slightly shorter, the $\pi$ bonding is weakened due to the lessened overlap of the $p_{z}$ orbitals and the curvature gives the partially bound atom a natural bias out-of-plane ${ }^{41,42}$. This different conformation makes the system less reactive, since now the water molecule can saturate readily only one "dangling bond". 
We have identified several stable intermediate states inspired by the analogy with the planar graphene case and found important differences in the reaction mechanisms solely induced by curvature effects. In the initial physisorbed state $(N-I)$, shown in Figure 5.4a, the molecule is much closer to the vacancy than in the planar case. From there, the water molecule has to overcome higher barriers compared to the planar case to reach the intermediate stable states, since the system is less reactive, and most importantly the molecule can not spontaneously dissociate over three "dangling bonds" (as in the planar geometry for IM4 and $K$ states).

However, if the oxygen atom saturates the $\mathrm{C}_{3}$ "dangling bond" first, as in the straight dissociation in graphene, the energetics for production of hydrogen are much more favorable. The direct dissociation proceeds with a low energy barrier compared to the planar case, of about $18 \mathrm{kcal} / \mathrm{mol}$.

To illustrate the reaction mechanism for the direct decomposition reaction we show in Fig. 5.4 the transition state geometry (N-TST, Fig. 5.4c) and the final stable state with oxygen bonded to the $\mathrm{C}_{3}$ atom (N-F, Fig. 5.4d) while leaving the five-fold ring untouched. This configuration is not the only final state that the system can reach. An analogue of the $F$ state, namely with the $\mathrm{O}$ atom in the middle of the vacancy site, can be reached through pathways that involve intermediate configurations. In these other reaction paths, first one hydrogen atom saturates the $\mathrm{C}_{3}$ "dangling bond", and then the $\mathrm{OH}$ group completes the $s p^{3}$ carbon bonding leading to the exact analogue of the planar IM2 state, (N-IM2 in Fig. 4b). The energy barrier for this reaction is about $20 \mathrm{kcal} / \mathrm{mol}$. Contrary to the graphene case, this first configuration is the precursor of all the other possible intermediate states. The reconstructed bond in the five-fold ring has to be broken to reach, for instance, the $N-K$ and $N-I M 4$ states. This subsequent activation barrier is of the order of $\sim 25 \mathrm{kcal} / \mathrm{mol}$. From there, the dissociation reaction proceeds along similar lines as in the planar case. On the other hand, production of hydrogen from the N-IM2 state is quite unfavorable $\left(E_{A} \sim 70\right.$ 
$\mathrm{kcal} / \mathrm{mol}$ ). A final comment on the analogue of the $E$ state, $N-E$ : even though it is still the global minimum of the PES, as in the planar case, now the reaction barrier for the water splitting from this state is substantially lower, about $46 \mathrm{kcal} / \mathrm{mole}$ vs. $94 \mathrm{kcal} / \mathrm{mole}$ in graphene. It is thus clear that curvature can improve the energetics for the water dissociation reaction over a vacancy and drastically enhance the selectivity of the $\mathrm{H}_{2}$ production process.

Table 5.1. Activation energies for the forward $\left(E_{A, f}\right)$ and backward $\left(E_{A, r}\right)$ reactions and total energy change $(\Delta E)$ for each of the reaction steps in planar graphene.

\begin{tabular}{cccc}
\hline Reaction & $E_{A, f}(\mathrm{kcal} / \mathrm{mol})$ & $E_{A, r}(\mathrm{kcal} / \mathrm{mol})$ & $\Delta E(\mathrm{kcal} / \mathrm{mol})$ \\
\hline Bulk & 131 & 16 & 115 \\
$I \rightarrow I M 1$ & 16 & 24 & -8 \\
$I \rightarrow I M 2$ & 33 & 44 & -11 \\
$I \rightarrow I M 3$ & 15 & 56 & -41 \\
$I \rightarrow I M 4$ & 3 & 68 & -65 \\
$I \rightarrow K$ & 3 & 79 & -76 \\
$I M I \rightarrow F$ & 16 & 47 & -31 \\
$I M I \rightarrow I M 2$ & 28 & 31 & -3 \\
$I M I \rightarrow I M 3$ & 4 & 37 & -33 \\
$I M I \rightarrow E$ & 28 & 107 & -79 \\
$I M I \rightarrow K$ & 3 & 71 & -68 \\
$I M 2 \rightarrow F$ & 58 & 85 & -27 \\
$I M 2 \rightarrow K$ & 21 & 86 & -65 \\
$I M 2 \rightarrow E$ & 38 & 114 & -76 \\
$I M 3 \rightarrow I M 2$ & 55 & 25 & 30 \\
$I M 3 \rightarrow K$ & 14 & 49 & -35 \\
$I M 3 \rightarrow F$ & 112 & 110 & 2 \\
$I M 3 \rightarrow E$ & 60 & 106 & -46 \\
$I M 4 \rightarrow F$ & 80 & 53 & 27 \\
$I M 4 \rightarrow K$ & 13 & 24 & -11 \\
$K \rightarrow E$ & 72 & 83 & -11 \\
$K \rightarrow F$ & 49 & 12 & 37 \\
$E \rightarrow F$ & 94 & 46 & 48 \\
Straight & 55 & 94 & -39 \\
\hline
\end{tabular}




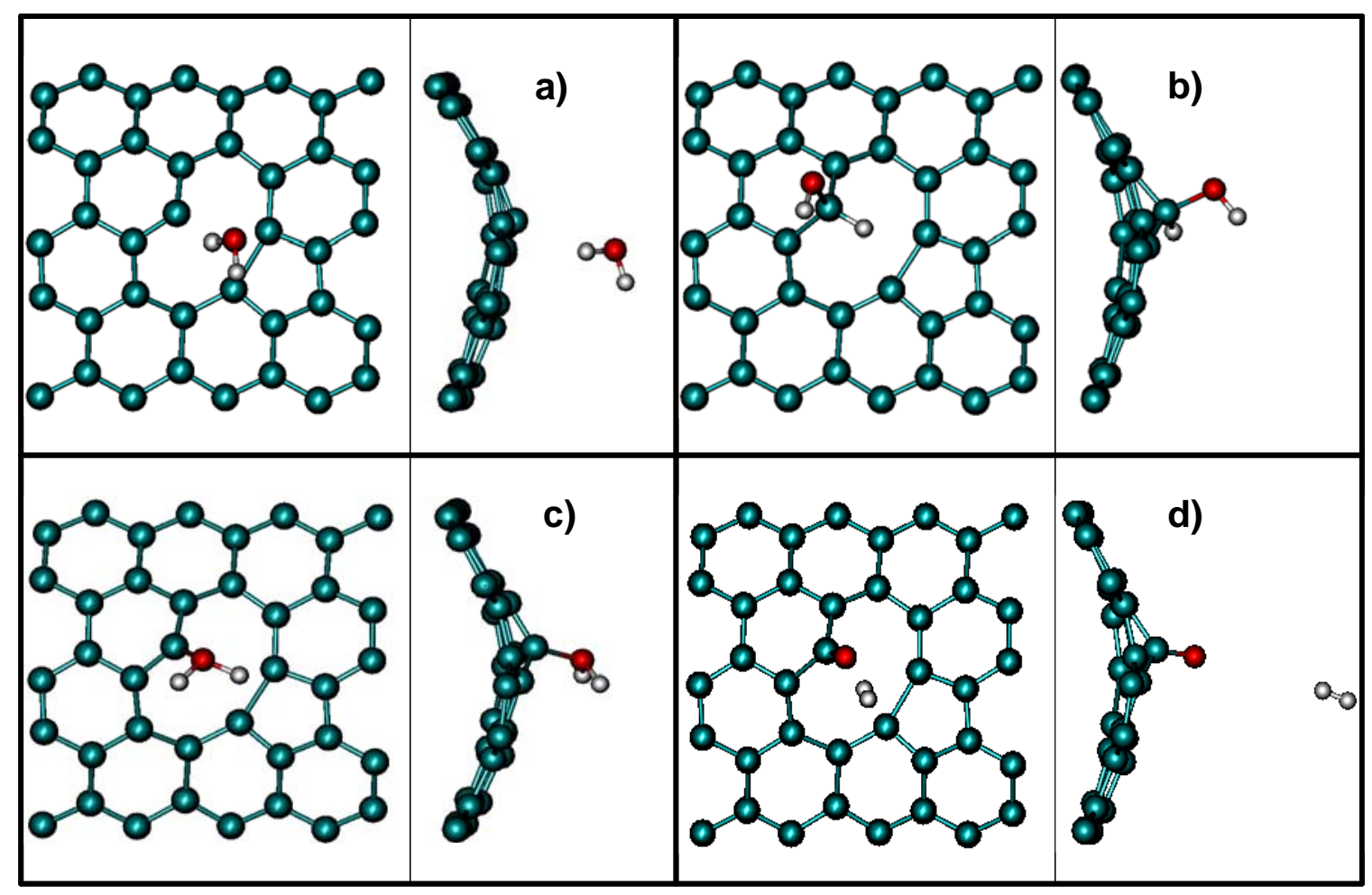

Figure 5.4. Top and side views of (a) the initial (N-I), (b) intermediate (N-IM2), (c) transition state $(N-T S T)$, and (d) final $(N-F)$ conformations for a favorable dissociation pathway over a vacancy in a $(10,10)$ nanotube.

For a quantitative assessment of the efficiency of the reaction, we estimated the equilibrium yield for the different cases of direct thermolysis, dissociation on a vacancy in graphene and in the nanotube, using the calculated PES and the vibrational frequencies of the relevant configurations. From the data displayed in Fig. 5.1 it is clear that the defective carbon substrate improves dramatically the equilibrium yield of $\mathrm{H}_{2}$ with respect to the dissociation in free space. This means that defects in graphitic materials would catalyze the dissociation of water with at least a factor of 2 gain in temperature with respect to the direct thermolysis, that becomes competitive only well above $2000 \mathrm{~K}$. A more detailed analysis of these thermodynamical data will be published elsewhere. ${ }^{43}$ 
Finally, we would like to point out that this reaction mechanism, as presented, is a batch process: once the vacancies in the carbon structure get saturated with oxygen, the process stops. It is then necessary to take the oxygen out to regenerate the active sites to make this a true catalytic process. There are several possibilities to achieve this, such as photoexcitation, or further reaction with other molecules. If such a process could be found, the energy needed to create the defects in the first place would be a one-time expenditure, and the system would thereafter work as a molecular energy conversion cycle: a true nanoscale chemical reactor. 


\section{References and Notes}

${ }^{1}$ A. Hashimoto, K. Suenaga, A. Gloter, K. Urita, S. Iijima, Nature 430, 7002 (2004).

${ }^{2}$ A.A. El-Barbary, First Principles Characterisation of Defects in Irradiated Graphitic Materials, Ph.D. Thesis, University of Sussex (2005).

${ }^{3}$ M.S. Dresselhaus, G. Dresselhaus, P.C. Eklund, Science of Fullerenes and Carbon Nanotubes, Academic Press, San Diego (1995).

${ }^{4}$ J.A. Turner, Science 305, 972 (2004).

${ }^{5}$ A. Kogan, Int. J. Hydrogen Energy 25, 1043 (2000).

${ }^{6}$ A.C. Dillon, K.M. Jones, T.A. Bekkedahl, C.H. Klang, D.S. Bethune, and M.J. Heben, Nature 386, 377 (1997).

${ }^{7}$ L. Schlapbach and A. Züttel, Nature 414, 353 (2001).

${ }^{8}$ A.C. Dillon and M.J. Heben, Appl. Phys. A 72, 133 (2001).

${ }^{9}$ R. G. Parr, W. Yang, Density-Functional Theory of Atoms and Molecules (Oxford Univ. Press, Oxford, 1989).

${ }^{10}$ E. E. Santiso, K. E. Gubbins, Mol. Simul. 30, 699 (2005)

${ }^{11}$ E.E. Santiso, M.K. Kostov, A.M. George, M. Buongiorno Nardelli, K.E. Gubbins, Appl. Surf. Sci. 253, 5570 (2007).

${ }^{12}$ M.K. Kostov, E.E. Santiso, A.M. George, K.E. Gubbins, M. Buongiorno Nardelli, Phys. Rev. Lett. 95, 136105 (2005).

${ }^{13}$ E.E. Santiso, L. Huang, K.E. Gubbins, M.K. Kostov, A.M. George and Marco Buongiorno Nardelli, “Ab initio simulations of chemical reactions in nanostructured carbon materials", in Quantum Chemical Calculations of Surfaces and Interfaces of Materials, American Scientific Publishers, Valencia, California (2007). 
${ }^{14}$ It is to be noted that the reactions compared in figured 5.1 have different end products (other than hydrogen). The main purpose of this figure is only to compare the ability of both processes to produce hydrogen from water.

${ }^{15}$ A. Hashimoto et al., Nature 430, 870 (2004).

${ }^{16}$ K. Nordlund, J. Keinonen, T. Mattila, Phys. Rev. Lett. 77, 699 (1996).

${ }^{17}$ J. R. Hahn, H. Kang, Phys. Rev. B 60, 6007 (1999).

${ }^{18}$ R. G. Parr, W. Yang, Density-Functional Theory of Atoms and Molecules (Oxford Univ. Press, Oxford, 1989).

${ }^{19}$ E. E. Santiso, K. E. Gubbins, Mol. Simul. 30, 699 (2005)

${ }^{20}$ D. Vanderbilt, Phys. Rev. B 41, 7892 (1990)

${ }^{21}$ CPMD, Copyright IBM Corp. 1990-2003, Copyright MPI für Festkörperforschung Stuttgart 1997-2001, http://www.cpmd.org

${ }^{22}$ PWSCF, S. Baroni, A. Dal Corso, S. de Gironcoli and P. Giannozzi, http://www.pwscf.org .

${ }^{23}$ A. D. Becke, Phys. Rev. A 38, 3098 (1988).

${ }^{24}$ C. Lee, W. Yang, R. G. Parr, Phys. Rev. B 37, 785 (1988).

${ }^{25}$ J.P. Perdew, K. Burke, and M. Ernzerhof, Phys. Rev. Lett. 77, 3865 (1996).

${ }^{26}$ A. Laio, M. Parrinello, Proc. Natl. Acad. Sci. U.S.A. 99 , 12562 (2002).

${ }^{27}$ M. Iannuzzi, A. Laio, and M.Parrinello, Phys. Rev. Lett. 90 , 238302 (2003).

${ }^{28}$ G. Mills, H. Jónsson, G. K. Schenter, Surf. Sci. 324, 305 (1995).

${ }^{29}$ H. Jónsson, G. Mills, K. W. Jacobsen, in Classical and Quantum Dynamics in Condensed Phase Simulations, B. J. Berne, G. Ciccotti, D. F. Coker, Eds. (World Scientific, Singapore, 1998) pp. 385-404.

${ }^{30}$ G. Henkelman, B. P. Uberuaga, H. Jónsson, J. Chem. Phys. 113, 9901 (2000).

${ }^{31}$ A. Banerjee, N. Adams, J. Simons, R. Shepard, J. Phys. Chem. 89, 52 (1985).

${ }^{32}$ S. R. Billeter, A. Curioni, W. Andreoni, Comp. Mater. Sci. 27, 437 (2003). 
${ }^{33}$ Our calculated standard enthalpy of formation of ideal gas water at zero $\mathrm{K}$ is $-51.75 \mathrm{kcal} / \mathrm{mole}$, and the free energy of formation at $298 \mathrm{~K}$ is $-206.3 \mathrm{kcal} / \mathrm{mole}$. Experimental values for the same quantities are -57.76 $\mathrm{kcal} / \mathrm{mol}$ and $54.59 \mathrm{kcal} / \mathrm{mol}$, respectively (from ref. 37). Our estimates are within $6 \mathrm{kcal} / \mathrm{mol}$ of the experimental values, which is to be expected of plane-wave BLYP calculations.

${ }^{34}$ T.M. Reed, K.E. Gubbins, “Applied Statistical Mechanics: Thermodynamic and Transport Properties of Fluids", McGraw-Hill, New York, 1973; see also E.E. Santiso and K.E. Gubbins, Molecular Simulation 30, 699 (2004).

${ }^{35}$ A.A. El-Barbary, R.H. Telling, C.P. Ewels, M.I. Heggie, and P.R. Briddon, Phys. Rev. B 68, 144107 (2003).

${ }^{36}$ P.A. Thrower, and R.M. Mayer, Phys. Status Solidi A 47, 11 (1978).

${ }^{37}$ National Institute of Standards and Technology (NIST) online data base.

${ }^{38}$ A. A. El-Barbary, R. H. Telling, C. P. Ewels, M. I. Heggie, P. R. Briddon, Phys. Rev. B 68, 144107 (2003).

${ }^{39}$ R. H. Telling, C. P. Ewels, A. A. El-Barbary, M. I. Heggie, Nature Mat. 2, 333 (2003).

${ }^{40}$ D. Townsend et al., Science 306,1161 (2004).

41 J.-C. Charlier et al., IEEE Trans. Nanotechnology 2(4), 349 (2003).

${ }^{42}$ P.M. Ajayan, V. Ravikumar, J.-C. Charlier, Phys. Rev. Lett. 81, 1437 (1998).

${ }^{43}$ E.E. Santiso, M.K. Kostov, K.E. Gubbins and M. Buongiorno Nardelli, in preparation. 


\section{Chapter 6}

\section{Conclusions and Future Research Directions}

The examples shown in this work illustrate the three main mechanisms in which a reaction can be affected by confinement: geometric restrictions due to steric hindrance, and physical and chemical interactions with the confining material. In our study of rotational isomerizations we have shown that, when the characteristic dimensions of the adsorbent are comparable to the molecular size, the equilibrium and kinetics of the reactions can be radically altered, with rates changing by many orders of magnitude within narrow ranges of pore sizes. It is remarkable that this shape-catalytic effect can be even more important than the effect of actual chemical interactions with the adsorbing material, depending on the reaction considered ${ }^{1}$.

Our study of the formaldehyde dissociation in confinement illustrates the effect that physical interactions with the support can have on both the equilibrium and kinetics of a chemical reaction. Although the effect on the reaction barrier in this case is small in relation to the barrier height, this type of effect can be important for reactions with smaller barriers, especially if charge transfer is involved ${ }^{2}$. The effect on the equilibrium distribution of reactants and products is, however, more important, and we find that, at different temperatures, the most favorable adsorbing material for the dissociation is different.

Finally, in our last example, we have seen how a chemically activated graphene surface can be used to induce the dissociation of hydrogen from water at temperatures lower than would be required for the bulk decomposition. This case also shows how geometry has a dramatic effect on the equilibrium distribution: by making the graphene surface curved, we change the temperature at which the hydrogen production peaks by about $1000 \mathrm{~K}$. From 
these examples it is clear that, by tuning the geometry of a catalytic support, it would be possible to design materials with much improved yields and selectivities.

For the reactions studied in this work, we have computed rates and equilibrium constants at infinite dilution. For porous materials of widths comparable to the molecular dimensions it is likely, however, that the density of the adsorbed phase also has an impact on the kinetics and thermodynamics of the reactions involved. We are currently developing a simulation protocol based on the Reaction Path Hamiltonian (RPH) approach ${ }^{3-8}$ to coarse-grain the nonreactive degrees of freedom and include intermolecular interactions. This will be used to study the impact of the thermodynamic state of the adsorbed phase on the chemical reactions, which is an additional factor to consider in the design of improved catalytic materials.

For the dissociation of water over defective carbons, we are currently studying the interaction of additional water molecules and oxygen atoms with the oxygen-saturated vacancy. This will allow us to ascertain whether this process can be used for hydrogen production. As an extension of this project, we are currently studying the interaction of water and other small molecules (such as $\mathrm{CH}_{4}, \mathrm{CO}$ and $\mathrm{CO}_{2}$ ) with other defective graphene surfaces, such as edges ${ }^{9-11}$, doped ${ }^{12}$, and functionalized carbons ${ }^{13}$. These systems are more accessible to experimental studies than single vacancies ${ }^{11,14-16}$, and can be used to continually dissociate carbon-containing molecules. We expect that the exploration of such systems, and the effect of geometric changes and physical interactions on them, will lead us to a protocol for the rational design of highly efficient carbon-based catalytic materials that take advantage of many catalytic effects simultaneously.

Finally, it is also important to consider the mechanism by which the reacting molecules enter and leave the porous space, as the diffusion process is also likely to have an impact on the overall dynamics of the system. We are currently adapting some of the newer 
simulation techniques available for such studies (in particular the Reaction Ensemble Molecular Dynamics method ${ }^{17,18}$ ) to consider the combined diffusion and reaction processes and gain insight on the influence of the porous network on the overall effectiveness of the catalytic materials under study.

We have illustrated in this work how a systematic study of each individual effect of confinement on reaction rates and reaction equilibrium can shed light on the fundamental nature of such effects. Our current efforts are geared toward using the information from these examples to construct a theoretical framework that can be used for the systematic nano-scale level design of improved catalytic materials. We believe that, with the current rate of improvements on the synthesis of carbon-based meso- and nano-structured materials ${ }^{11,19-25}$, it will soon be possible to systematically build such "super-catalysts" that take advantage of all the effects described above for much improved catalytic performance. 


\section{References and Notes}

\footnotetext{
${ }^{1}$ Some of the material in this chapter has been published in: Erik E. Santiso, Liping Huang, Keith E. Gubbins, Milen K. Kostov, Aaron M. Georg and Marco Buongiorno Nardelli, “Ab initio simulations of chemical reactions in nanostructured carbon materials”, in Quantum Chemical Calculations of Surfaces and Interfaces of Materials, American Scientific Publishers, Valencia, California (2007).

${ }^{2}$ M.D. Halls, H.B. Schlegel, J. Phys. Chem. B 106, 1921 (2002) .

${ }^{3}$ B. Peters, A.T. Bell, A. Chakraborty, J. Chem. Phys. 121, 4453 (2004).

${ }^{4}$ J. González, X. Giménez, J.M. Bofill, Theor. Chem. Acc. 112, 75 (2004).

${ }^{5}$ J. González, X. Giménez, J.M. Bofill, J. Phys.Chem. A 105, 5022 (2001).

${ }^{6}$ M. Page, J.W. McIver, Jr., J. Chem. Phys. 88, 922 (1988).

${ }^{7}$ W.H. Miller, B.A. Ruf, Y.-T. Chang, J. Chem. Phys. 89, 6298 (1988).

${ }^{8}$ W.H. Miller, N.C. Handy, J.E. Adams, J. Chem. Phys. 72, 99 (1980).

${ }^{9}$ D. Jiang, B.G. Sumpter, S. Dai, J. Chem. Phys. 126, 134701 (2007).

${ }^{10}$ J.F. Espinal, A. Montoya, F. Mondragón, T.N. Truong, J. Phys. Chem. B 108, 1003 (2004).

${ }^{11}$ R.E. Smalley, Y. Li, V.C. Moore, B.K. Price, R. Colorado, Jr., H.K. Schmidt, R.H. Hauge, A.R. Barron, J.M. Tour, J. Am. Chem. Soc. 128, 15824 (2006).

${ }^{12}$ Y.-H Kim, Y. Zhao, A. Williamson, M.J. Heben, S.B. Zhang, Phys. Rev. Lett. 96, 016102 (2006).

${ }^{13}$ Y. Zhao, Y.-H. Kim, A.C. Dillon, M.J. Heben, S.B. Zhang, Phys. Rev. Lett. 94, 155504 (2005).

${ }^{14}$ J.C. Meyer, A.K. Geim, M.I. Katsnelson, K.S. Novoselov, T.J. Botth, S. Roth, Nature 446, 60 (2007).

${ }^{15}$ K. Murata, M. Yudasaka, S. Iijima, Carbon 44, 799 (2006)

${ }^{16}$ M.J. Bronikowski, P.A. Willis, D.T. Colbert, K.A. Smith, R.E. Smalley, J. Vac. Sci. Technol. A 19, 1800 (2001).

${ }^{17}$ J.K. Brennan, M. Lísal, K.E. Gubbins, B.M. Rice, Phys. Rev. E 70, 061103 (2004)

${ }^{18}$ M. Lísal, J.K. Brennan, W.R. Smith, F.R. Siperstein, J. Chem. Phys. 121, 4901 (2004)
} 
${ }^{19}$ S. Lei, J.-I. Miyamoto, T. Ohba, H. Kanoh, K. Kaneko, J. Phys. Chem. C 111, 2459 (2007).

${ }^{20}$ T. Kyotani, Z. Ma, A. Tomita, Carbon 41, 1451 (2003).

${ }^{21}$ Z. Ma, T. Kyotani, A. Tomita, Carbon 40, 2367 (2002).

${ }^{22}$ Z. Ma, T. Kyotani, Z. Liu, O. Terasaki, A. Tomita, Chem. Mater. 13, 4413 (2001).

${ }^{23}$ S.H. Joo, S. Jun, R. Ryoo, Microporous Mesoporous Mater. 44, 153 (2001).

${ }^{24}$ S. Jun, S.H. Joo, R. Ryoo, M. Kruk, M. Jaroniec, Z. Liu, T. Ohsuna, O. Terasaki, J. Am. Chem. Soc. 122, $10712(2000)$.

${ }^{25}$ R. Ryoo, S.H. Joo, S. Jun, J. Phys. Chem. B 103, 7743 (1999). 